Portland State University

PDXScholar

Fall 1-1-2012

\title{
Understanding the Emission from Semiconductor Nanoparticles
}

\author{
Beth Ann Manhat \\ Portland State University
}

Follow this and additional works at: https://pdxscholar.library.pdx.edu/open_access_etds

Part of the Other Chemistry Commons

Let us know how access to this document benefits you.

\section{Recommended Citation}

Manhat, Beth Ann, "Understanding the Emission from Semiconductor Nanoparticles" (2012).

Dissertations and Theses. Paper 465.

https://doi.org/10.15760/etd.465

This Dissertation is brought to you for free and open access. It has been accepted for inclusion in Dissertations and Theses by an authorized administrator of PDXScholar. Please contact us if we can make this document more accessible: pdxscholar@pdx.edu. 
Understanding the Emission from Semiconductor Nanoparticles

by

Beth Ann Manhat

A dissertation submitted in partial fulfillment of the requirements for the degree of

\author{
Doctor of Philosophy \\ in \\ Chemistry
}

Dissertation Committee:
Andrea Goforth, Chair
Shankar Rananavare
Dean Atkinson
Tania Vu
Rolf Könenkamp

Portland State University

2012 
C2012 Beth Ann Manhat 


\begin{abstract}
This dissertation describes the synthesis and characterization of fluorescent semiconductor nanoparticles (NPs) in order to optimize their biomedical utility for imaging and sensing applications. While both direct and indirect bandgap semiconductor NPs have been studied, control over their emission properties vary. Quantum confinement (QC), which primarily controls the emission wavelength of nanosized semiconductors, dictates that as the size of semiconductor NPs decrease, the magnitude of the bandgap increases, resulting in changes in the observed emission wavelength: smaller NPs have a larger bandgap, and thus a bluer emission. However, surface, interfacial, or shell defects can act as non-radiative or radiative recombination sites for excitons formed within the NP; the latter results in emission competition with the bandgap transition, as described Chapters 1 and 2 .
\end{abstract}

Because the emission wavelengths of direct bandgap semiconductor NPs correlate with size according to the expectations of QC, and are stable in aqueous environments with high quantum efficiencies (quantum yield, QY), current research focuses on their potential biomedical applications. Chapter 3 describes red-emitting $\mathrm{CdSe} / \mathrm{ZnS}$ quantum dots (QDs) that exhibit a concentration-dependent decrease in fluorescence intensity in response to the neurotransmitter serotonin (5-hydroxytryptamine, 5-HT). A mechanistic study was performed to understand a 5-HT-dependent decrease in QD emission and calibration curves relating QD intensity loss to 5-HT concentration in ensemble and single QD studies were generated. 
Unfortunately, the known toxicity of CdSe-based QDs has generated interest in more benign semiconductor NPs to replace these QDs in biological applications, while maintaining the same degree of control over the emission color and QY. Bulk indirect bandgap semiconductors, such as $\mathrm{Si}$, have low efficiency inter-band transitions, and $\mathrm{Si}$ NPs are known to contain radiative defects that can alter the emission wavelength from QC-based size expectations; these competitive emission pathways must be controlled in order for Si NPs to be successfully used in biological applications. In general, synthetic methods that gives precise control over both the particle size and surface termination are needed in order to produce emission controlled Si NPs. Relative to groups II and VI QDs, synthetic routes to prepare Si NPs are few in numbers, and the size vs. defect emission events are difficult to assign. Not only do these assignments vary amongst reports, but they also vary with particle size, solvent, sample age, and identities of the surface ligands.

Si NPs have been prepared through two synthetic routes using the Zintl salt, sodium silicide $(\mathrm{NaSi})$ and ammonium bromide $\left(\mathrm{NH}_{4} \mathrm{Br}\right)$ as precursors. Chapter 4 describes the synthesis performed in the solvent $\mathrm{N}, \mathrm{N}$,-dimethylformamide (DMF). This reaction produces blue-emitting Si NPs $(5.02 \pm 1.21 \mathrm{~nm})$ that bear partial hydride surface termination. However, it was determined that the solvent was able to interact with the $\mathrm{Si}$ NP surface, and prevent subsequent functionalization. This observation was used advantageously, and Chapter 5 describes a one-pot Zintl salt metathesis of Si NPs (3.9 \pm $9.8 \mathrm{~nm}$ ) performed in a bi-functional (amine or carboxylic acid) solvent ligand, where the observations indicated that the solvent ligands coordinate to the Si NP. 
The emission maxima of the Si NPs prepared from the Zintl salt metathesis exhibited a dependence on the excitation energy, and is indicative of emission that is influenced by QC, which likely originates from deeply oxide embedded 1-2 nm crystalline cores. The Si NPs prepared from the one-pot Zintl salt metathesis were exposed to metals salt ions of varying reduction potentials to determine the band edges by what will or will not be reduced (Chapter 6). By monitoring the emission intensity of the Si NPs, in addition to the UV-Vis of the metal ions, the band edge of Si NPs may be determined. The value of the band edge may lend insight into the origin of Si NP emission.

To utilize fluorescent Si NPs for biological applications, red emission is strongly preferred. Unfortunately, when preparing aqueous Si NPs, red emission usually changes to blue, likely from the oxidation of the Si NP surface. Therefore, the red emission needs to be efficiently protected from surface oxidants. Because both increased chain lengths and steric modalities have been found to protect the emission properties of Si NPs, redemitting, ester-functionalized Si NPs $(5.51 \pm 1.35 \mathrm{~nm})$ with varying chain lengths and ester termination moieties were prepared to determine the best method of preserving the observed red emission in the presence of potential alcoholic oxidants. By determining the best was to protect Si NPs emission, the red-emission from Si NPs may be preserved for biological applications. 
To all those who supported me, and more importantly, To all those who didn't! 


\section{Acknowledgments}

It is my great pleasure to acknowledge the help and encouragement to those I am grateful; their assistance throughout this process was not unnoticed!

To my advisor, Dr. Andrea Goforth, I extend my deepest gratitude and appreciation for your endless direction and mentoring. I believe a good mentor walks a fine line between giving directions and waiting to see what happens - Andrea, thank you for walking this line so well. I feel honored to have been your first graduate student, and know that I have benefited from your eternal optimism and extensive scientific knowledge. There is no doubt that your influence will continue to have an impact on my career.

To my committee members, I appreciate you "sitting on another committee" and for offering suggestions. A special thanks to Dr. Shankar Rananavare for being one of the most "pro-student" faculty members I have ever met! Your advice has been vital.

To the Goforth lab, past and present, I am grateful for all the hours of scientific (and nonscientific) discussion.

To "the gang," I cannot believe you guys actually listened to all my rants and raves; thank you for pulling me out of the lab, whether I needed it or not!

To my grandparents, thank you for your unwavering support, both emotionally and financially! My grandfather has inspired me to ask questions, while my grandmother 
taught me not to accept the answers at face value. As difficult as it was, you raised me to be confident enough to move across the country and earn my Ph.D.

Finally, to my soon-to-be husband, Shawn, I cannot say thank you enough for your immense support, encouragement, and love. You were lucky enough to miss my late nights in the lab, but you have understood the time I needed to take to get my work done now: thanks for always doing the dishes I did not have enough time to do! I love you. 


\section{Table of Contents}

$\begin{array}{lc}\text { Abstract } & \text { i } \\ \text { Dedication } & \text { iv } \\ \text { Acknowledgments } & \mathrm{v} \\ \text { List of Tables } & \text { xii } \\ \text { List of Figures } & \text { xiii } \\ \text { List of Abbreviations and Symbols } & \text { xvii }\end{array}$

Chapter 1: General Introduction to Semiconductor Nanoparticles for Biomedical Applications $\quad 1$

$\begin{array}{ll}1.1 \text { General Introduction } & 2\end{array}$

1.2 Structure of Dissertation 4

Chapter 2: Quantum Confinement and Photoluminescence in Semiconductor Nanoparticles for Biological Applications $\quad 7$

2.1. Electronic Structure of Materials $\quad 8$

2.2. Quantum Confinement (QC) in Semiconductors 9

2.3 Emission from Direct and Indirect Bandgap Semiconductor NPs 13

2.3.1 Direct Bandgap Semiconductor NPs $\quad 13$

2.3.2 Indirect Bandgap Semiconductor NPs 15

2.4 Biological Application of Nanomaterials 18

2.4.1 Optical Properties for Biological Fluorophores 19

2.4.2 Emission from Organic Dyes and Fluorescent NPs 20

Chapter 3: Single CdSe/ZnS Quantum Dot Sensors for Quantification of Serotonin Concentration in Localized, Live Cell Nano-environments

3.1 Introduction 25

$\begin{array}{ll}3.2 \text { Experimental } & 27\end{array}$

3.2.1 Mechanistic Determination 28 
3.3 Results and Discussion

3.3.1 Photophysical Investigation of Ensemble-QD Response to $5-\mathrm{HT}$

3.3.2 Mechanistic Determination of QD-5-HT Quenching

3.3.3 Degradation of the QDs by Core/Shell Decomposition or other 5-HT-Accelerated Atomic Rearrangements

3.3.4 Förster Resonance Energy Transfer (FRET) between the QDs and 5-HT

3.3.5 Photoinduced Charge $\left(\mathrm{e}^{-}\right.$or $\left.\mathrm{h}^{+}\right)$Transfer (PCT) between the QDs and 5-HT

3.3.5.1 QD Excited State Lifetimes

3.3.5.2 Stern Volmer (SV) Analysis of the QD-5-HT Interaction

3.4 Quantitative Calibration of Single, 5-HT Receptor-Targeted QDs in Response to 5-HT Concentration in a Live Cell Environment

3.5 Discussion on QD-5-HT Quenching Mechanism and Development of Neurotransmitter-Specific Nanosensors

3.6 Conclusions

\section{Chapter 4: Synthesis of Functionalized Si NPs using a Two-Step Metathesis} and Hydrosilylation Reaction

4.1 Introduction $\quad 54$

4.2 Si NP Experimental and Characterization 56

4.2.1 Si NP Synthesis $\quad 56$

4.2.2 Hydrosilylation (HS) of the prepared Si NPs 57

$\begin{array}{ll}\text { 4.2.3 Si NP Characterization } & 58\end{array}$

$\begin{array}{ll}4.3 \text { Results and Discussion } & 58\end{array}$

4.3.1 DMF-synthesized Si-H NPs Prepared from the Metathesis of $\mathrm{NaSi}$ and $\mathrm{NH}_{4} \mathrm{Br}$ 
4.3.2 Heat-catalyzed HS Reaction of the DMF-synthesized Si-H NPs using 1-decene

4.3.3 Chemically-Catalyzed HS Reaction the DMF-synthesized Si-H NPs using 1-decene

4.3.4 Heat-Catalyzed HS Reaction of Xylene-synthesized Si-H NPs and 1-decene

4.4 Conclusions

\section{Chapter 5: One-Step Synthesis for Water Soluble Photoluminescent Si}

Nanoparticles

76

$\begin{array}{ll}\text { 5.1 Introduction } & 77\end{array}$

5.2 Si NP Experimental and Characterization $\quad 79$

$\begin{array}{ll}\text { 5.2.1 Si NP Synthesis } & 79\end{array}$

$\begin{array}{ll}\text { 5.2.2 Si NP Characterization } & 81\end{array}$

$\begin{array}{ll}5.3 \text { Results and Discussion } & 85\end{array}$

5.3.1 Characterization of Glutaric Acid (GA)-synthesized Si NPs $\quad 86$

5.3.2 Citric Acid (CA)- and ethylenediamine (en)-synthesized Si NP Characterization and Photophysical Properties 97

5.4 Discussion of the Origin of Si Photophysical Properties 105

5.5 Cellular Properties of GA-synthesized Si NPs 109

$\begin{array}{lr}5.6 \text { Conclusions } & 110\end{array}$

Chapter 6: Using Silicon Nanoparticles as Reducing Agents for Metal Ions 112

$\begin{array}{ll}6.1 \text { Introduction } & 113\end{array}$

$\begin{array}{ll}6.2 \text { Experimental } & 115\end{array}$

$\begin{array}{ll}\text { 6.2.1 Synthesis of Si NPs } & 115\end{array}$

6.2.2 Photophysical Study Using the Si NPs and Metal Ions 116

6.2.3 Analytical Characterization of the Si NP and Metal Ions 117

6.3 Photochemical Reactions of the blue-emitting GA-Si NPs with Various Metal Ions 
6.3.1 Characterization of the GA-Si NPs

6.3.2 Photostability of the neat GA-Si NPs

6.3.3 Photochemical Reaction of the GA-Si NPs with $\mathrm{AuCl}_{4}{ }^{-}$

6.3.4 Photochemical Reaction of the GA-Si NPs with $\mathrm{Ag}^{+}$

6.4 Photochemical Reactions of the red-emitting $\mathrm{SiO}_{\mathrm{x}}-\mathrm{Si}$ NPs with Various Metal Ions

6.4.1 Characterization of the neat $\mathrm{SiO}_{\mathrm{x}}-\mathrm{Si} \mathrm{NPs}$

6.4.2 Photostability of the $\mathrm{SiO}_{\mathrm{x}}-\mathrm{Si}$ NPs

6.4.3 Photochemical Reaction of the $\mathrm{SiO}_{\mathrm{x}}-\mathrm{Si} \mathrm{NPs}$ with $\mathrm{AuCl}_{4}{ }^{-}$

6.4.4 Photochemical Reaction of the $\mathrm{SiO}_{\mathrm{x}}-\mathrm{Si} \mathrm{NPs}$ with $\mathrm{Ag}^{+}$

6.5 Discussion

6.6 Conclusions

Chapter 7: Synthesis and Emission Properties of Red-Emitting, EsterFunctionalized Silicon Nanoparticles

7.1 Introduction

7.2 Si NP Experimental and Characterization

7.2.1 Synthesis of the bi-Functional Esters

7.2.2 Synthesis of Ester-Functionalized Si NPs

7.2.3 Alcohol Dispersions of the Ester-Functionalized Si NPs

7.2.4 Characterization of the bi-Functional Esters and EsterFunctionalized Si NPs

7.3 Results and Discussion

7.3.1 Analytical Characterization of the Ester-Functionalized Si NPs 
8.2 General Discussion and Future Prospects 166

$\begin{array}{ll}\text { Terminal References } & 169\end{array}$

\section{Appendices}

Appendix A: Supporting Information for Introduction Chapters

Appendix B: Supporting Data and Radical Determination for CdSe/ZnS QDs as Serotonin Nanosensors

Appendix C: Supporting Information for the Zintl Salt Metathesis of $\mathrm{NaSi}$ and $\mathrm{NH}_{4} \mathrm{Br}$

Appendix D: Additional Metal Ions and Molecules for Quenching GA-Si NP Emission

Appendix E: Full Characterization of the Ester-Functionalized Si NPs 


\section{List of Tables}

Table 2.1 Calculated Exciton Bohr Radii $\left(r_{B}\right)$ for Various Semiconductors 11

Table 2.2 Emission Range and QY values for Organic and Semiconductor Fluorophores

Table 3.1 Average Intensity-weighted lifetimes $(\tau)$ of QDs $_{593}$ in the presence of 5-HT

Table 6.1 GA-Si NP $+\mathrm{HAuCl}_{4}{ }^{-}$photochemical reactions and the effects on the SPR band formation

Table 7.1 Si NP size and diffraction patterns from the ester-functionalized Si NPs

Table 7.2 Emission Maxima ( $\lambda_{\mathrm{ex}}=370 \mathrm{~nm}$, unless noted $)$ of the esterfunctionalized Si NPs 


\section{List of Figures}

Figure 2.1 Scheme of the electronic structures of bulk semiconductors, insulators, and metals.

Figure 2.2 Scheme of semiconductor energy levels as a function of material size. Bulk semiconductors, nanoparticles, and molecules are compared.

Figure 2.3 Scheme of direct and indirect bandgap semiconductors in reciprocal $(\mathrm{k})$ space.

Figure 2.4 Emission spectra $\left(\lambda_{\mathrm{ex}}=350 \mathrm{~nm}\right)$ of $\mathrm{CdSe} / \mathrm{ZnS}$ QDs as a function of particle size.

Figure 2.5 Models Explaining the Potential Origin of Photoluminescence in Si NPs.

Figure 2.6 Fluorescent dyes of varying chain lengths with corresponding emission ranges and QYs.

Figure 3.1 Scheme of red-emitting CdSe@ZnS QDs coated in TOPO and PAA with corresponding QD size; digital images of $10 \mathrm{nM}$ QDs before and after exposure to $100 \mu \mathrm{M}$ 5-HT.

Figure 3.2 Absorbance and steady-state PL spectra $\left(\lambda_{\mathrm{ex}}=340 \mathrm{~nm}\right)$ of $\left(\mathrm{QDs}_{593}\right)$

Figure 3.3 Emission spectra $\left(\lambda_{\mathrm{ex}}=340 \mathrm{~nm}\right)$ and ICP-MS analysis of QD 585 +5 -HT and control QD 585 .

Figure 3.4 5-HT absorption spectrum (200-600 nm) and $\mathrm{QD}_{593}$ emission spectrum $\left(\lambda_{\mathrm{ex}}=370 \mathrm{~nm}\right.$, emission from $\left.380-700 \mathrm{~nm}\right)$ showing no spectral overlap.

Figure 3.5 Excited state lifetime decays for $\mathrm{QD}_{580}$ solutions exposed to (a) $0 \mu \mathrm{M}$, (b) $100 \mu \mathrm{M}$, (c) $350 \mu \mathrm{M}$, and (d) $1230 \mu \mathrm{M}$ 5-HT.

Figure 3.6 SV plot showing a non-linear relationship between the average intensity-weighted lifetime ratio, $\tau_{\mathrm{o}} / \tau$, of $\mathrm{QDs}_{580}$ and 5-HT concentrations up to $1200 \mu \mathrm{M}$.

Figure 3.7 Modified SV plot illustrating the linear relationship of 5-HT concentrations and the average lifetime ratios of QDs580.

Figure 3.8 Single QD nanosensors, targeted to 5-HT receptors in a live cell environment quantitatively calibrated to 5-HT concentration down to $10 \mathrm{nM}$. 
Figure 3.9 Schematic of CdSe/ZnS QDs undergoing dynamic loss/gain of surface ligands.

Figure 3.10 Relative energy levels of red-emitting CdSe@ZnS QD valance and conduction bands for nanosensors relative to possible analyte, including 5-HT and DA

Figure 4.1 Scheme of the Zintl metathesis to prepare Si NPs 59

Figure 4.2 Photophysical properties of the DMF-Si NPs 61

Figure 4.3 TEM image of DMF-synthesized Si NPs 62

Figure 4.4 FT-IR spectra of neat DMF and DMF-synthesized Si-H NPs 63

Figure 4.5 Emission spectra of the hexane and water layers extracted from the HS of the DMF-synthesized Si NPs with 1-decene

Figure 4.6 TEM images of the extracted hexane and water layers of Si NPs from the HS of the DMF-synthesized Si-H NPs with 1-decene 65

Figure 4.7 FT-IR spectra of the water and hexane extractions from the heatcatalyzed HS of the DMF-synthesized Si-H NPs with 1-decene

Figure 4.8 A. Emission using $\lambda_{\mathrm{ex}}=250 \mathrm{~nm}$ (solid) and $\lambda_{\mathrm{ex}}=280 \mathrm{~nm}$ (dashed) and B. FT-IR spectra comparing the hydrophilic Si NPs from the heat-(black) and chemically-(blue) catalyzed HS reaction

Figure 4.9 A. Emission using $\lambda_{\mathrm{ex}}=250 \mathrm{~nm}$ (solid) and $\lambda_{\mathrm{ex}}=280 \mathrm{~nm}$ (dashed) and B. FT-IR spectra comparing the hydrophobic Si NPs from the heat-(black) and chemically-(blue) catalyzed HS reactions

Figure 4.10 Emission and FTIR spectra of the hydrophobic Si NPs from the Zintl salt metathesis and HS reaction in xylene.

Figure 5.1 Representative one-step melt synthesis of Si NPs in GA

Figure 5.2 TEM images of the GA-Si NPs

Figure 5.3 Raman spectra of freshly prepared GA-synthesized Si NPs

Figure 5.4 XRD pattern of GA-synthesized Si NPs 90

Figure 5.5 XPS scans of the GA-synthesized Si NPs 91

Figure 5.6 Fingerprint regions of the FT-IR spectra for neat GA, freshly prepared, purified Si NPs, and aged, purified Si NPs

Figure 5.7 Zeta potential of the GA-synthesized Si NPs as a function $\mathrm{pH}$ 
Figure 5.8 Emission spectra of GA-synthesized Si NPs in $\mathrm{H}_{2} \mathrm{O}$

Figure 5.9 TEM of (a) CA- and (b) en-synthesized Si NPs 98

Figure 5.10 Fingerprint regions of the FT-IR spectra of (a) neat CA and

(b) CA-synthesized Si NPs

Figure 5.11 Fingerprint regions of the FT-IR spectra of (a) neat en and (b) en-synthesized Si NPs.

Figure 5.12 Zeta potential of the (a) CA-synthesized and (b) en-synthesized $\mathrm{Si}$ NPs as a function of $\mathrm{pH}$

Figure 5.13 Digital image of agarose gel under $302 \mathrm{~nm}$ light after electrophoresis of the luminescent Si NPs

Figure 5.14 Absorbance and emission spectra of neat, as-prepared (A) CA-synthesized, (B) en-synthesized Si NPs

Figure 5.15 Time-resolved emission decay spectrum of GA-synthesized Si NPs excited at $375 \mathrm{~nm}$

Figure 5.16 GA-synthesized Si NPs incubated with N2a cells to monitor cellular uptake

Figure 6.1 Optical properties of the neat, diluted GA-Si NPs

Figure 6.2 Absorption spectra of neat $\mathrm{HAuCl}_{4}$ and the $\mathrm{GA}-\mathrm{Si} \mathrm{NPs}+\mathrm{HAuCl}_{4}$ photochemical reaction

Figure 6.3 Emission spectra of the GA-Si NPs $+\mathrm{HAuCl}_{4}$ photochemical reaction

Figure 6.4 TEM images of the Au NPs resulting from the GA-Si NP + $\mathrm{HAuCl}_{4}$ photochemical reaction

Figure 6.5 EDX spectrum of the NPs in the GA-Si $\mathrm{NP}+\mathrm{HAuCl}_{4}$ photochemical reaction

Figure 6.6 XRD pattern of the products of the GA-Si NPs $+\mathrm{HAuCl}_{4}$ photochemical reaction

Figure 6.7 Photophysical properties of GA-Si NPs $+\mathrm{HAuCl}_{4}$ solution not exposed to UV light

Figure 6.8 Absorption spectra of neat $\mathrm{AgNO}_{3}$ and the GA-Si NPs $+\mathrm{AgNO}_{3}$ photochemical reaction 
Figure 6.9 Emission spectra of the GA-Si NPs $+\mathrm{AgNO}_{3}$ photochemical reaction

Figure 6.10 TEM images of the Ag NPs resulting from the GA-Si NPs + $\mathrm{AgNO}_{3}$ photochemical reaction

Figure 6.11 Absorbance and emission spectra of the GA-Si NPs $+\mathrm{AgNO}_{3}$ not exposed to UV light

Figure 6.12 Absorbance and emission spectra of the $\mathrm{SiO}_{\mathrm{x}}-\mathrm{Si} \mathrm{NP}+\mathrm{HAuCl}_{4}$ photochemical reaction

Figure 6.13 TEM images of $\mathrm{Au}$ NPs resulting from the $\mathrm{SiO}_{\mathrm{x}}-\mathrm{Si} \mathrm{NPs}+\mathrm{HAuCl}_{4}$ photochemical reaction

Figure 6.14 Absorbance spectra of the $\mathrm{SiO}_{\mathrm{x}}-\mathrm{Si} \mathrm{NPs}+\mathrm{AgNO}_{3}$

Figure 7.1 Scheme of the synthesis of the bi-functional esters and the estersynthesized Si NPs

Figure 7.2 FTIR spectra of (a) tert-butyl-4-pentenoate- (5T), (b) tert-butyl-10decenoate- (11T), (c) methyl-4-pentenoate- (5M), and (d) methyl -10-undecenoate- (11M) Si NPs.

Figure 7.3 Representative TEM images of the 11M-Si NPs

Figure 7.4 PXRD pattern of the 11M-Si NPs compared to the calculated PXRD pattern of diamond lattice $\mathrm{Si}$

Figure 7.5 Representative UV-Vis absorption spectra of the ester-synthesized Si NPs

Figure 7.6 Representative emission spectrum of the 5M-Si NPs

Figure 7.7 Emission spectra of 5M-Si NPs dispersed in methanol, pentanol, and decanol

Figure 7.8 Emission spectra of 11M-Si NPs dispersed in methanol, pentanol, and decanol

Figure 7.9 Emission spectra of 5T-Si NPs dispersed in methanol, pentanol, and decanol 


\section{List of Abbreviations and Symbols}

\begin{tabular}{|c|c|}
\hline$\Delta \mathrm{E}_{\mathrm{g}}$ & bandgap \\
\hline $5-\mathrm{HT}$ & 5-hydroxytryptamine hydrochloride \\
\hline a.u. & arbitrary units \\
\hline $\mathrm{CA}$ & citric acid \\
\hline $\mathrm{CB}$ & conduction band \\
\hline $\mathrm{CdSe}$ & cadmium selenide \\
\hline DMF & $\mathrm{N}, \mathrm{N}$,-dimethylformamide \\
\hline EDX & energy dispersive X-ray spectroscopy \\
\hline em & emission \\
\hline en & ethylenediamine \\
\hline ex & excitation \\
\hline FT-IR & Fourier Transmission Infrared \\
\hline GA & glutaric acid \\
\hline HS & hydrosilylation \\
\hline k-space & reciprocal (momentum) space \\
\hline $\mathrm{LC}$ & luminescent center \\
\hline $\mathrm{nm}$ & nanometer \\
\hline NP & nanoparticle \\
\hline QC & quantum confinement \\
\hline QD & quantum dot \\
\hline QY & quantum yield \\
\hline PCT & photoinduced charge transfer \\
\hline PL & photoluminescence \\
\hline
\end{tabular}


$\mathrm{r}_{\mathrm{B}}$

SAED

$\mathrm{Si}$

SPR

TEM

UV

VB

Vis

XPS

XRD
Bohr exciton radius

selected area electron diffraction

silicon

surface plasmon resonance

transmission electron microscopy

ultra violet

valance band

visible

X-ray photoelectron spectroscopy

X-ray diffraction 


\title{
General Introduction to Semiconductor Nanoparticles for Biomedical Applications
}

\begin{abstract}
Nanotechnology blends the concepts of science and engineering in order to understand and utilize materials in the 1-100 $\mathrm{nm}$ size range. Novel optical properties from small semiconducting nanoparticles (NPs) have generated interest in controlling and applying these properties in new applications. The optical properties of semiconductor NPs can change as a function of particle size, as dictated by the quantum confinement (QC) effect. This general introduction chapter describes the favorable and unfavorable properties of direct and indirect bandgap semiconductor NPs, specifically as they relate to their use in potential biological applications. The overall focus of this dissertation is presented, as well as brief descriptions of the following chapters.
\end{abstract}




\subsection{General Introduction}

Nanoscience and nanotechnology blend the concepts of physics, chemistry, biology, and engineering with the intention of understanding and utilizing materials in the 1-100 nm size range; colloidal nanoparticles (NPs, "nano-" $=10^{-9} \mathrm{~m}$ ) are comprised of 100s-1000s of atoms (e.g., a 1-nm Au NP contains about 250 atoms). While NPs have been used over the ages, it is only in the last 30-40 years that researchers have been able to control their synthesis using two general methods ${ }^{[1]}$ : "top down" and "bottom up". Top down synthesis methods use larger, bulk (often $\mu \mathrm{m}$-sized) starting materials from which atoms are removed to leave nanosized particles; the bottom up approaches involve the assembly of individual atoms, which cluster together to form NPs. Once high quality, size-controlled NPs were produced, new properties were observed, including novel optical properties from small, semiconducting NPs. Similar to bulk semiconductors, the photophysical properties of semiconductor NPs are believed to originate from the recombination of charge carriers across the semiconductor bandgap. However, unlike bulk, a variety of emission colors have resulted from different sized NPs of the same material. The trend of optical properties that change as a function of size is controlled by the quantum confinement (QC) effect. ${ }^{[2]}$ As a semiconductor decreases in size from bulk to nano, more atoms are removed, and this affects the magnitude of the bandgap. Therefore, a controllable shift in the bandgap is observed for increasingly small semiconductor particles: smaller NPs have larger bandgaps with blue shifted emission. Both direct and indirect bandgap semiconductor nanocrystals have been shown to follow QC, with emission ranging from the NIR to UV. ${ }^{[3]}$ However, it has been found that 
indirect bandgap NPs, such as Si, also exhibit a surface or defect-related recombination that can affect the observed emission wavelength. ${ }^{[4]}$ With the goal of utilizing fluorescent NPs for various applications in the technological ${ }^{[5]}$ and biomedical $^{[6]}$ fields, understanding and controlling the optical properties of quantum-confined semiconductor NPs is imperative.

Even with the challenges associated with controlling the emission wavelength of indirect bandgap semiconductor NPs, research on the biological utility of NPs is promising, as evidenced by the dramatic rise in published reports over the last two decades. ${ }^{[7]}$ The optical properties of semiconductor NPs are favorable for in vitro and in vivo labeling, tracking, and sensing applications, based on their narrow emission and broad absorption profiles, ${ }^{[8]}$ a high quantum efficiency (or quantum yield, QY), ${ }^{[8]}$ and significant resistance to photobleaching as compared to organic dyes. ${ }^{[9]}$ The small diameter of NPs is not only important for their biological utility, but it also gives NPs a high surface-to-volume ratio that is ideal for subsequent surface modifications. Using classical organic chemistry techniques, researchers have focused on producing NPs with surface groups that can impart the NPs with aqueous solubility and potential bioconjugation sites, while preserving the desired emission properties. However, direct bandgap semiconductors, such as group II-IV and III-IV quantum dots (QDs), have highly emissive and controllable optical properties that correlate with semiconductor size, these materials also possess a known toxicity that has inspired the use of semiconductor shells and polymeric coatings to increase their biological use. ${ }^{[10]}$ This potential toxicity has also made Si NPs, an abundant and benign material, attractive for replacing group II- 
IV and III-IV QDs in possible biological applications. Unfortunately, to be used biologically, Si NP emission must be better understood and controlled. Until such time, the biological utility of Si NPs will be limited.

\subsection{Structure of Dissertation}

The focus of this thesis is directed towards understanding the fluorescence of inorganic semiconducting NPs for potential biological application. Using standard UVVis and photoluminescence spectroscopy, the emission properties of direct and indirect bandgap semiconductor nanomaterials are examined in order to maximize their utility in biological sensing and labeling. The reactivity of Si NPs in the presence of analytes has also been examined to gain insight into their electronic reactivity and stability.

Chapter 1 is a general description as to the interest of nanosized semiconductor fluorophores, and provides information on the favorable and unfavorable photophysical properties of semiconductor NPs for biological applications.

Chapter 2 is a more in depth theoretical chapter, detailing the origin of the QC effect and provides information on the emission mechanism of both direct and indirect semiconductor NPs. Chapter 2 also provides a universal description of the requirements for biological fluorophores, as well as a comparison of the emission properties of semiconductor NPs and organic dyes.

Chapter 3 is an experimental chapter detailing the motivation and study of $\mathrm{CdSe} / \mathrm{ZnS}$ (core/shell) QDs as single-QD nanosensors to detect the neurotransmitter serotonin. 
Included here are experiments related to elucidating the mechanism of the 5-HTconcentration dependent decrease of CdSe/ZnS QD emission intensity.

Chapter 4 is an experimental chapter detailing the two-step Zintl salt metathesis to prepare hydride- and decane-functionalized $\mathrm{Si}$ NPs. When the synthesis and functionalization of the Si NPs was performed in a coordinating solvent, competition between the solvent and subsequent ligand for the Si surface sites was observed, and thus experiments aimed at minimizing this competition were examined.

Chapter 5 is an experimental chapter detailing the development of a one-pot Zintl salt metathesis for generating water-soluble, fluorescent, Si NPs in various bi-functional solvent ligands. The Si NP phase, surface, and photophysical properties were examined, alongside a biological imaging study.

Chapter 6 is an experimental chapter describing the interaction of blue- and red-emitting Si NPs with metal ions to determine the band edges of the Si NPs. The Si NP emission spectra and the metal ion absorption spectra were collected in order to understand their interaction, which may help lend insight into the reduction potentials of the Si NPs.

Chapter 7 is an experimental chapter describing the synthesis and characterization of red-emitting ester-functionalized Si NPs from the hydrosilylation of bi-functional esters and hydride-terminated Si NPs. The chain length and size of the terminating ester moiety show varying levels of preserving the observed red emission of the Si NPs in the presence of varying alcohol solvents. 
Chapter 8 is a summary chapter detailing the major conclusions from the experimental chapter, alongside personal views and recommendations for further research. 


\title{
Quantum Confinement and Photoluminescence in Semiconductor Nanoparticles for Biological Applications
}

\begin{abstract}
Bulk semiconductors are comprised of a high number of atoms and energy levels, which are separated by a bandgap generally ranging from a few tenths of an $\mathrm{eV}$ to $4 \mathrm{eV}$. When a bulk semiconductor is reduced in size to a nanosized semiconductor, the bandgap shifts to larger values due to the quantum confinement (QC) of excitons into more discrete energy levels rather than continuous bands; exciton confinement results in size-controlled photophysical properties of semiconductor nanoparticles (NPs). This chapter describes the derivation of the QC effect, which has been observed in both direct and indirect bandgap semiconductor particles. This chapter also describes the alternate mechanisms that have been developed to explain the emission from indirect bandgap semiconductor NPs, such as Si NPs. Finally, the requirements for biological imaging and sensing agents are presented, including a comparison of the photophysical properties of fluorescent semiconductor NPs and commonly used fluorescent organic dyes.
\end{abstract}




\subsection{Electronic Structure of Materials}

Bulk solids correspond to matter on the macroscopic scale, which are comprised of a large number of atoms whose energy levels form a continuum because they have very similar energy values in the crystal lattice of ordered atoms. The continuum of the highest energy levels filled with electrons constitutes the valence band (VB), while a continuum of the lowest unoccupied energy levels constitutes the conduction band (CB). The relative positions of the $\mathrm{VB}$ and the $\mathrm{CB}$ are related to the material composition, and the magnitude of their energetic separation is known as the bandgap, $\Delta \mathrm{E}_{\mathrm{g}}$. The value of the bandgap also classifies materials into three types: (1) metals, which have no $\Delta \mathrm{E}_{\mathrm{g}}$ due to the overlap of the $\mathrm{CB}$ and $\mathrm{VB}$; (2) insulators, where the $\Delta \mathrm{E}_{\mathrm{g}}$ is large (generally $>4$ $\mathrm{eV}$ ); and (3) semiconductors, where the $\Delta \mathrm{E}_{\mathrm{g}}$ varies from several tenths of an $\mathrm{eV}$ to $4 \mathrm{eV}$ (Figure 2.1). ${ }^{[1]}$ The Fermi level, also shown in Figure 2.1, defines the highest occupied electronic level (in chemistry, known as the HOMO), above which all energy levels are unoccupied at $0 \mathrm{~K}^{[1]}$ 


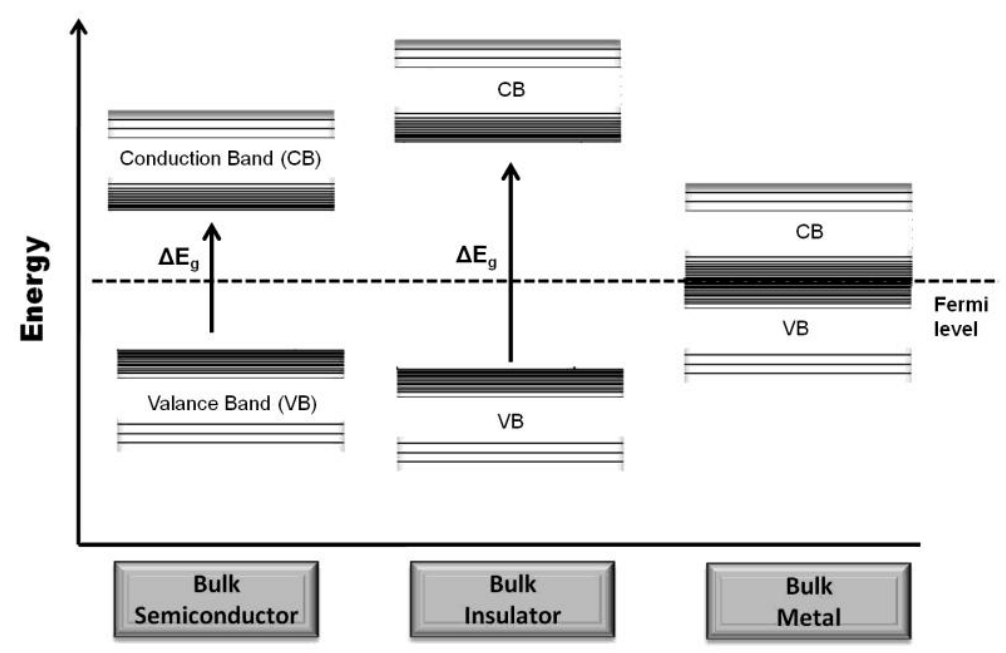

Figure 2.1. Scheme of the electronic structures of bulk semiconductors, insulators, and metals.

\subsection{Quantum Confinement (QC) in Semiconductors}

Bulk semiconductors are comprised of a high number of repetitive atom arrays with densities of charge carriers in the $10^{9}-10^{21} \mathrm{~cm}^{-3}$ range. ${ }^{[1]}$ As illustrated in Figure 2.2, the $\Delta \mathrm{E}_{\mathrm{g}}$ is the energy required for $\mathrm{VB}$ electrons to be (thermally or photonically) excited to the $\mathrm{CB}$. The excited electron in the $\mathrm{CB}$ and resulting hole in the $\mathrm{VB}$ are known as an exciton pair, and are separated by a distance known as the Bohr exciton radius $\left(\mathrm{r}_{\mathrm{B}}\right)$. Assuming neither finds a competitive decay pathway, the exciton recombination results in the release of energy (light or heat) approximately equal to that of the $\Delta \mathrm{E}_{\mathrm{g}}$; it is the efficiency and tunability of this emission that has prompted interest in semiconductor nanomaterials. 


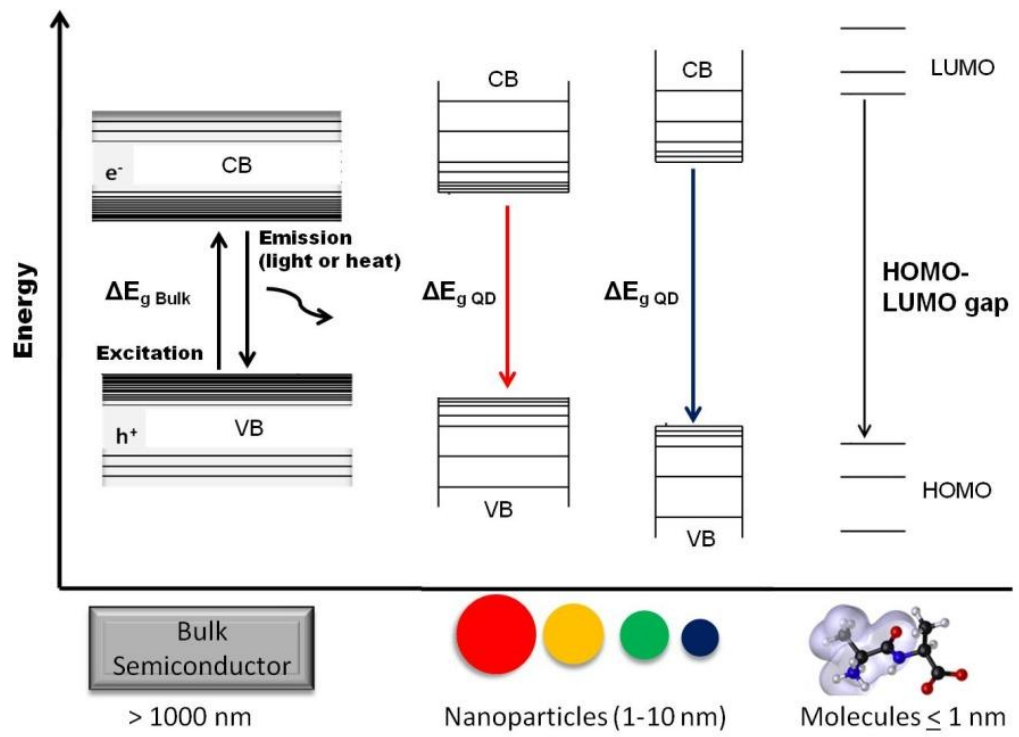

Figure 2.2. Scheme of semiconductor energy levels as a function of material size. Bulk semiconductors, nanoparticles, and molecules are compared.

The physical effect of changing a bulk semiconductor into a nanosized semiconductor involves decreasing the number and density of the energy levels, which leads to changes in the $\Delta \mathrm{E}_{\mathrm{g}}$. As a nanocrystal decreases in size, exciton confinement ${ }^{[12]}$ occurs as the particle size approaches the $\mathrm{r}_{\mathrm{B}}$, which varies by the semiconductor (Table 2.1). The consequence of this confinement is that the continuous energy bands start to resemble increasingly more discrete (or quantized) energy levels that satisfy the "particle in a box model" and the Schrodinger equation ${ }^{[13]}$ (See Appendix for derivation). 
Table 2.1. Calculated Exciton Bohr Radii $\left(r_{B}\right)$ for Various Semiconductors. ${ }^{[13]}$

\begin{tabular}{cc}
\hline Material & $\mathrm{r}_{\mathrm{B}}(\mathrm{nm})$ \\
\hline $\mathrm{ZnO}$ & 1.8 \\
$\mathrm{ZnS}$ & 5.0 \\
$\mathrm{Si}$ & 5.5 \\
$\mathrm{CdSe}$ & 6.1 \\
$\mathrm{CdTe}$ & 10.0 \\
$\mathrm{PbS}$ & 20.4 \\
$\mathrm{InAs}$ & 34.0 \\
$\mathrm{PbSe}$ & 46.0 \\
\hline
\end{tabular}

The relative positions of the energy bands in nanosized semiconductors vary with the degree of confinement, and thus as a function of semiconductor nanocrystallite size. In a quantum dot (QD), excitons are confined in three dimensions, such that the energy may be described using a three-dimensional potential well. ${ }^{[1]}$ Within the well, the potential energy is zero while it is infinite at the edges. The energy of excitons within a spherical QD ${ }^{[14]}$ can be defined by Equation 2.1,

$$
E_{3 D \text { well }}=\frac{h^{2}}{2 m d^{2}}
$$

where $\mathrm{h}$ is Plank's constant, $\mathrm{m}$ is the mass of the particle, and $\mathrm{d}$ is the diameter of the QD sphere. While the minimum energy to create an exciton pair is approximately equal to the bulk bandgap $\left(\Delta \mathrm{E}_{\mathrm{g} \text { bulk }}\right)$, the exciton confinement energy $\left(\mathrm{E}_{\mathrm{conf}}=E_{\text {well }_{e^{-}}}+E_{\text {well }_{h^{+}}}\right)$ must also be considered. For larger particles where $d>r_{B}$ (and $d$ approaches infinity), $\mathrm{E}_{\text {conf }}$ is approximately zero. However, for particles with $\mathrm{d} \leq \mathrm{r}_{\mathrm{B}}, \mathrm{E}_{\mathrm{conf}}$, also known as the zero point energy, can be estimated. This is the energy of the potential well at the lowest energy ${ }^{[14]}$, as given by Equation 2.2, 


$$
E_{w e l l}=\frac{h^{2}}{2 m^{*} d^{2}}
$$

where $\mathrm{m}^{*}$ is the reduced mass of the excitons $\left(\frac{1}{m^{*}}=\frac{1}{m_{e}}+\frac{1}{m_{h}}\right.$, where $\mathrm{m}_{\mathrm{e}}$ and $\mathrm{m}_{\mathrm{h}}$ are the effective masses for electrons and holes, respectively). Additionally, the Coulomb interaction $\left(\mathrm{E}_{\mathrm{Coul}}\right)$ of the excitons must also be considered in order to determine the bandgap confinement energy; $\mathrm{E}_{\mathrm{Coul}}$ accounts for the exciton and hole attraction and the crystal screening, ${ }^{[14]}$ as given by Equation 2.3,

$$
E_{\text {Coul }}=\frac{-1.8 e^{2}}{e \pi \varepsilon \varepsilon_{0} d}
$$

where $\varepsilon$ is the dielectric constant of the semiconductor material and $\varepsilon_{\mathrm{o}}$ is the vacuum permittivity, respectively. As illustrated by Brus ${ }^{[12]}$, the size-dependent energy gap of a spherical semiconductor $\mathrm{QD}$ is found by combining $\Delta \mathrm{E}_{\mathrm{g} \text { bulk }}$ and Equations 2.2 and 2.3,

$$
\Delta E_{g Q D}=\Delta E_{g \text { bulk }}+E_{\text {well }}+E_{\text {Coul }}=\Delta E_{g \text { bulk }}+\frac{h^{2}}{2 m^{*} d^{2}}-\frac{1.8 e^{2}}{2 \pi \varepsilon \varepsilon_{0} d}
$$

Equation 2.4 uses the effective mass approximation ${ }^{[1,12]}$, and is dominated by the $\mathrm{E}_{\text {well }}$ term $\left(d^{2}\right)$. Therefore, decreasing the particle size near the $r_{B}$ results in an exciton recombination that is controlled by a size-dependent energy shift, as illustrated in Figure. 2.2. ${ }^{[2]}$ This phenomenon, known as the quantum confinement (QC) effect, is used to explain the observed trends of luminescent NP properties: decreasing NP size has a larger effect on the bandgap, which blue shifts the emission energy. 


\subsection{Emission from Direct and Indirect Bandgap Semiconductor NPs}

The effects of QC have been observed in both direct and indirect bandgap semiconductor NPs. As bulk materials, direct and indirect bandgap semiconductors differ in their band structure in reciprocal (or momentum) space. ${ }^{[1-2]}$ In reciprocal space, energy is a function of the wave vector, $\mathrm{k}$, which is represented by Equation 2.3,

$k=\frac{2 \pi^{*} p}{h}$

where $\mathrm{p}$ is the charge carrier's momentum. Figure 2.3 illustrates the intrinsic differences between direct and indirect bandgaps structures in reciprocal space, which are treated as having parabolic energy bands. ${ }^{[12]}$
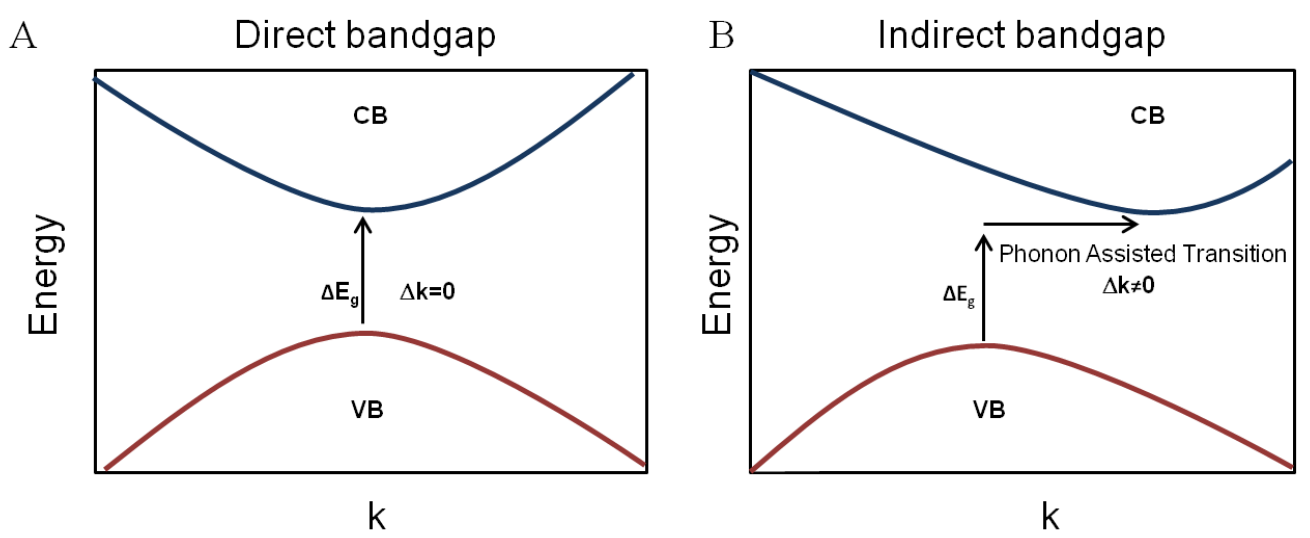

Figure 2.3. Scheme of direct (A) and indirect (B) bandgap semiconductors in reciprocal (k) space.

\subsubsection{Direct Bandgap Semiconductor NPs}

Direct bandgap semiconductors, such as CdSe and other group II-VI and III-VI materials, have energy gaps with the minimum of the $\mathrm{CB}$ and the maximum of the VB 
aligned in k-space (Figure 2.3A). Because photons do not have momentum, the direct bandgap optical transitions exhibit no change in $\mathrm{k}$, resulting in vertical transitions, which are allowable via the Franck-Condon Principle. These "allowable" transmissions give direct bandgap NPs transitions that follow the expectations from QC: decreasing size results in optical properties that shift to shorter wavelengths.

The emission from direct bandgap CdSe quantum dots (QDs) was first observed in the $1980 \mathrm{~s}^{[15]}$ when the observed optical properties were blue-shifted as compared to bulk CdSe. ${ }^{[12]}$ While these effects were generally explained by QC, much of the early work was limited by the production of polydisperse and polymorphic NPs with polychromatic emissions. ${ }^{[16]}$ A full series of size-controlled, monodisperse CdSe QDs was produced by Murray et al..$^{[3 b]}$ using the pyrolysis of organometallic reagents. Using this method, CdSe QD crystallites coated in tri-n-octylphosphine oxide (TOPO) ranging from 1.2-11.5 $\mathrm{nm}$ were obtained by arresting particle growth in hot solutions. Since then, core/shell structures (using wide bandgap semiconductors) have been developed to minimize toxicity and increase the optical efficiency of the fluorescent QD cores. Additionally, bi-functional molecules, such as mercaptoacetic acid or (poly)acrylic acid, have been used to render these QDs water-dispersible. Regardless of the presence of semiconductor shells, or organic molecules, it was found that as the CdSe QD core size decreases, the electronic transitions blue shift from 675 to $400 \mathrm{~nm}$ (Figure 2.4), consistent with the generation of smaller NPs with larger smaller bandgaps (as described by QC). 


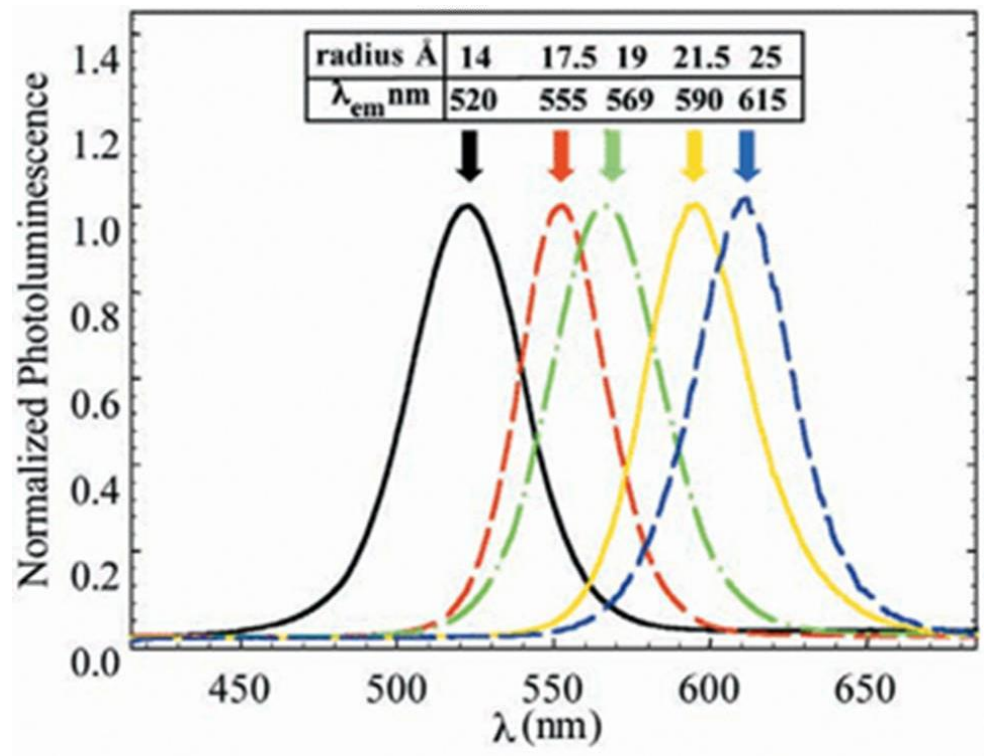

Figure 2.4. Emission spectra of $\mathrm{CdSe} / \mathrm{ZnS}$ QDs as a function of particle size $\left(\lambda_{\mathrm{ex}}=350 \mathrm{~nm}\right) \cdot{ }^{[17]}$

\subsubsection{Indirect Bandgap Semiconductor NPs}

Indirect bandgap semiconductors, such as $\mathrm{Si}$, do not have the minimum of the $\mathrm{CB}$ and maximum of the VB aligned in reciprocal space (Figure 2.3). An indirect bandgap across the bandgap requires the absorption of a phonon (vibrational energy), in addition to a photon, to accommodate the horizontal component of the transition. This results in a forbidden transition as described by the Franck-Condon Principle since $\Delta \mathrm{k} \neq 0$. Because electronic transitions in bulk Si are forbidden, this manifests itself in a more complicated emission scheme; specifically, as $\mathrm{Si}$, and other indirect bandgap semiconductors, decreases in size, shifted optical properties are not solely based on QC. 
Prior to 1990, it was believed that only direct bandgap semiconductors could efficiently emit in the visible. However, Canham ${ }^{[18]}$ observed visible luminescence from hydrofluoric acid (HF) treated Si wafers, which were later determined to possess small, narrow Si pores (or porous $\mathrm{Si}$ ). The absorption and emission shifts observed by porous $\mathrm{Si}$, and later for Si NPs, suggests that size-dependent optical properties are also present in indirect bandgap semiconductors. This report by Canham initiated the discussion as to the origin of emission from nanosized $\mathrm{Si}$, and led to three main exciton recombination mechanisms $^{[4]}$ to describe Si emission (Figure 2.5): (1) Quantum Confinement (QC); (2) Quantum Confined/Luminescent Center (QC/LC); and (3) Luminescent Center (LC). Similar to direct bandgap semiconductors, Si emission originating from QC involves the generation of an exciton pair from within the crystalline Si NP core that recombine and emit energy based on the $\Delta \mathrm{E}_{\mathrm{g}}$. The $\mathrm{LC}$ mechanism describes emission that is related to the formation and recombination of excitons from surface defects (e.g., dangling or incomplete bonding), which are often due to the presence of surface oxidation. The QC/LC emission mechanism also involves the generation of the excitons from within the Si NP cores; however, the presence of surface defect sites, often from surface oxide groups, can provide alternative decay pathways for recombination. 

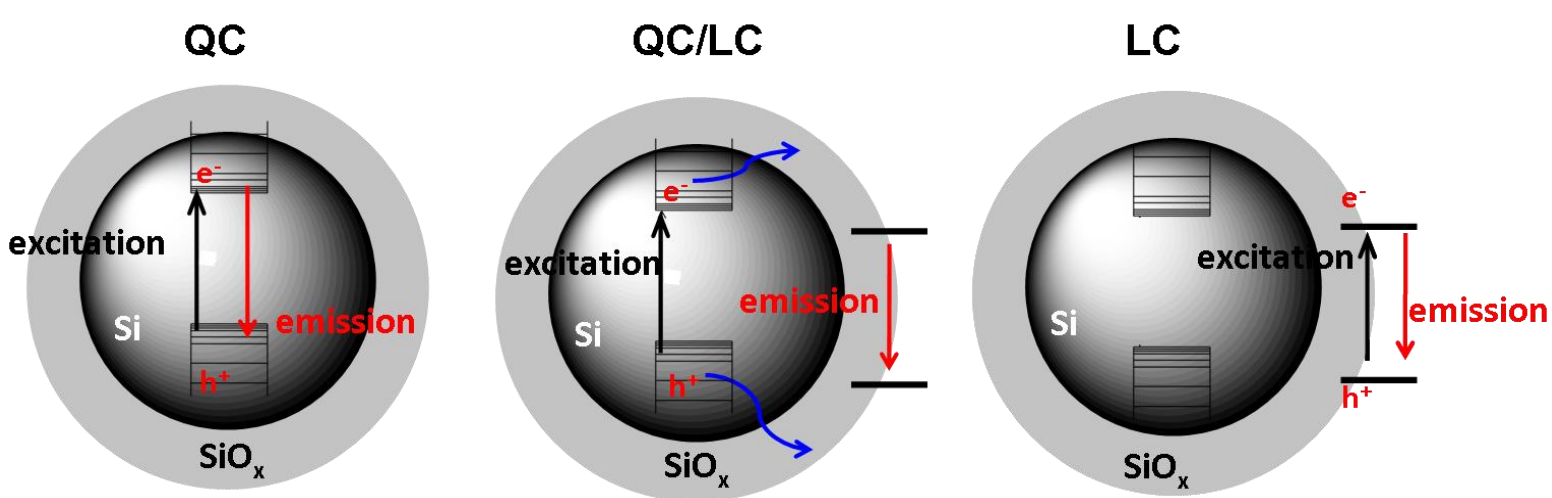

Figure 2.5. Models of the Potential Origin of Photoluminescence from Si NPs. QC-recombination describes the exciton recombination across the bandgap within the Si NP core. QC/LC-recombination describes emission resulting from exciton recombination from core excitons at a shell or surface sites. LC describes the excitation and emission from self-contained luminescence centers from a shell or surface.

It has been shown that that chemical etching can produce a size range of freestanding hydride-terminated Si NPs (1-10 nm) with a tunable emission in the visible region. ${ }^{[3 c, 19]}$ However, in order to use Si NPs practically, their surfaces will likely require functionalization. Different techniques for changing the surface groups (including hydrosilylation ${ }^{[20]}$, and Grignard reagents ${ }^{[21]}$ ) have successfully functionalized Si surfaces. The ability to manipulate the surface of the Si NP (and form covalent bounds) is advantageous for the use of Si NPs, but has often led to changes in the observed emission. It is believed that QC controls the emission of the hydride-terminated Si NPs, and that this is maintained when hydrocarbons are exchanged on the surface.

Attempts to generate aqueous compatible Si NPs have often led to shifts in the observed photophysical properties. It is hypothesized that exposing Si NP surfaces to oxygen (via water or other small, oxygenated molecules such as hydroxide,) can cause 
changes to the emission wavelengths, which are often blue shifts. Several reports have attributed the presence of a blue only emission event, or blue emission alongside other redder emission events, to localized $\mathrm{Si}-\mathrm{O}$ surface states consistent with the QC/LC mechanism. ${ }^{[22]}$ Furthermore, expectations from QC suggest that 1-2 nm Si NPs should emit blue ${ }^{[23]}$ however, blue emission from larger 4-nm Si NPs have also been reported. ${ }^{[3 c \text {, }}$ 23-24] The examples of the 4-nm blue-emitting aqueous Si NPs that have been reported ${ }^{[20 d,}$ ${ }^{25]}$ support the origin of blue emission from Si NPs arising from the QC/LC mechanism, with recombination occurring from outside of the bandgap. Maintaining QC emission in aqueous dispersions has been difficult, but controlling QC/LC emission has also not been accomplished. Regardless of the mechanism of nanosized Si emission, it needs to be controllable in order for Si NPs to be applied to specific applications.

\subsection{Biological Application of Nanomaterials}

Understanding complex biological systems depends on the ability to visualize them using high spatial and temporal resolution imaging agents, which allow detection on the molecular level. ${ }^{[8 \mathrm{~b}]}$ The high resolution $(\mathrm{ca} .50 \mathrm{~nm})$ of fluorescence makes it wellsuited for detecting molecular species. ${ }^{[26]}$ Current fluorescent materials studied for in vivo detection include molecular organic dyes, ${ }^{[27]}$ metal-ligand complexes, ${ }^{[28]}$ lanthanide chelate complexes, ${ }^{[29]}$ and more recently, semiconductor NPs. ${ }^{[6 \mathrm{~d}, 30]}$ Organic dyes have been used for biological imaging and sensing, owing to their small relevant size, wellcharacterized photophysical properties, and well-developed synthetic methods. ${ }^{[8 b]}$ 
However, because of the superior properties of nanoscaled fluorophores (vide infra), researchers are motivated to replace the use of dyes with high-quality, photostable fluorescent NPs. In the following sections, the biological requirements for biological imaging agents and sensors will be discussed, as well as a comparison of the photophysical properties of organic dyes and semiconductor NPs.

\subsubsection{Optical Properties for Biological Fluorophores}

Optical imaging has many advantages over other in vivo imaging techniques, as it is a real-time method for detecting bioactive molecules or molecular processes. ${ }^{[31]}$ The most encouraging advantage is that optical imaging uses non-ionizing radiation, making it a relatively safe imaging modality. Fluorescence imaging techniques utilize photoexcited fluorophores whose emission can be detected. Suitable in vivo fluorescence agents have many requisites, the first of which is an emission in the optically-transparent window. Because tissues have low absorption $\left(\mu_{\mathrm{a}}\right)$ and scattering $\left(\mu_{\mathrm{s}}\right)$ coefficients from 650-1200 nm (See Table A.1), a range in which most cellular media are not fluorescent, imaging handles with emission here are sought. Other spectral characteristics for biocompatible fluorophores include a substantive QY (at least $10 \%{ }^{[32]}$ ), a broad Stokes shift (large values suggest high sensitivity ${ }^{[33]}$ ), as well as photostability. And though not an optical property, proper functional groups are important for matrix compatibility, as well as potential site-specific binding, or sensing. 


\subsubsection{Emission from Organic Dyes and Fluorescent NPs}

Emission from organic dyes originates from molecular orbital transitions whose energy values and electronic positions are determined by the molecular structure. When photons are absorbed, electrons are promoted from the highest occupied molecular orbital (HOMO) to the lowest unoccupied molecular orbital (LUMO; see Figure 2.2, page 8). Upon relaxation, the electrons return to the ground state and the molecule releases energy. Since aromatic hydrocarbons and conjugated bonds can exhibit emissive pi-topi* transitions, organic dyes commonly utilize these molecular scaffolds. Therefore, fluorescent dyes (Figure 2.6, Table 2.2) have been synthesized with a variety of conjugated chain lengths with QY values and emission wavelengths spanning the UVNIR. ${ }^{[34][35] ~[36] ~[37] ~[38] ~}$

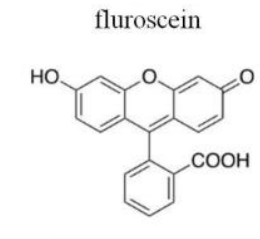

$500-550 \mathrm{~nm}$ QY 0.97

Cyanine 5

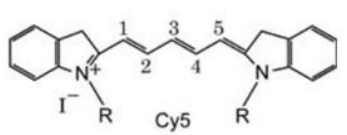

$600-675 \mathrm{~nm}$

QY 0.40

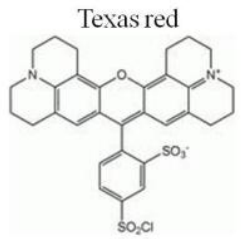

$550-600 \mathrm{~nm}$ QY 0.90

indocyanine green (ICG)

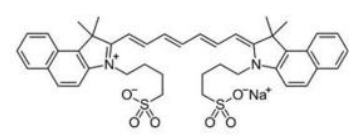

$800-825 \mathrm{~nm}$ QY 0.05

Figure 2.6. Fluorescent dyes of varying conjugated chain lengths with corresponding emission ranges and QYs. ${ }^{[8 \mathrm{~b}]}$ 
As described in Section 2.2, the optical properties of emissive semiconductor NPs are predominantly controlled by particle size. As dictated by QC, as the size of a NP decreases, the bandgap increases, and blue shifts the observed emission: fluorescent NPs possess a size-tunable emission. A wide range of organic dyes have been produced, which follow the general trend that increasing the conjugated bond system lowers the absorption energy and red-shifts the emission. ${ }^{[39]}$ NP absorption profiles are also broad, often spanning a majority of the visible wavelengths, and possess molar absorption coefficients up to 2 magnitudes larger than those of organic dyes, which have narrow absorption profiles. ${ }^{[40]}$ NPs also have more narrow emission profiles as compared to organic dyes, suggesting they may be useful for multicolor imaging. Additionally, it has been found that semiconductor NP QY, particularly of direct bandgap QDs, are high in the VIS and NIR. In contrast, organic dyes tend to emit strongly in the VIS with a reduced QY in the NIR (Figure. 2.6 and Table 2.2). This decreases the utility of organic dyes within the optically-transparent window.

Table 2.2. Emission Range and QYs for Organic and Semiconductor Fluorophores. ${ }^{[\mathrm{b}]}$

\begin{tabular}{ccc}
\hline Material & Emission Range (nm) & Absolute QY \\
\hline CdSe QDs & $450-650$ & $0.6-0.8$ \\
fluorescein & $500-550$ & 0.97 \\
Texas Red & $550-600$ & 0.9 \\
Cy5 & $600-675$ & 0.4 \\
CdTe QDs & $500-700$ & $0.4-0.7$ \\
ICG & $800-825$ & .04 \\
PbSe QDs & $900-1400$ & 0.5 \\
\hline
\end{tabular}


Because macromolecular dyes are built on aromatic scaffolds, the variety of functional groups has been a big advantage to their biological utility, and has led to a high number of functionalized or conjugated dyes for commercial purchase or facile synthesis. There are also established protocols for functionalizing, purifying, and characterizing dyes, as well as guides for dye bioconjugates. ${ }^{[41] ~[42] ~[43] ~ P r o t o c o l s ~ f o r ~ t h e ~}$ functionalization and subsequent purification of NPs, unfortunately, do not exist yet. However, conjugation of NP surface groups to molecules using carboimmide-activated coupling between amine and carboxylic acid groups, $\left.{ }^{[6]}\right]$ as well as melainimed-catalyzed coupling between amine and sulfhydryl groups have been performed on NP surfaces. ${ }^{\text {[6d-f, }}$ 44] Conventional chemical analyses such as FT-IR, NMR, and zeta potential are used to determine changes in NP surface groups to support the efficiency of such couplings. Additionally, these surface groups have acted as locations for energy and charge transfer in biological sensing or chemical reactions. ${ }^{[45]}$

Unfortunately, the emission profiles of organic fluorophores are greatly affected by media and light exposure. Changes in the media $\mathrm{pH}$ have led to aggregated-induced quenching of some dye emission profiles. ${ }^{[46]}$ Additionally, organic dyes exhibit low resistance to photodegradation and photobleaching, often occurring within minutes after exposure. ${ }^{[46,47]}$ While synthetic efforts toward producing more stable organic dyes have been put forth, ${ }^{[46 c]}$ there is still limited application for organic dyes based on this photoinstability and to date, only one dye (indocyanine green) has been approved for clinical use. $^{[48]}$ The stability of NPs at varying $\mathrm{pH}$ levels is based on the nature of the chemical surface coatings and the extent of surface coverage. CdSe-based QDs and Si NPs are 
often synthesized in organic solvents and both electrostatically and covalently surface modifiers are used to render the particles water-dispersible. These capping agents and functional groups dictate the particle-particle interactions, and the few studies to date on stability under light exposure show the potential for a large number of photons to interact with NPs before effects on the emission profiles are observed, which can potentially withstand irradiation up to a few hours. ${ }^{[9 a, 49]}$ This stability makes them well suited for temporal imaging and sensing applications using fluorescence as the mode of detection. 


\title{
Single CdSe/ZnS Quantum Dot Sensors for Quantification of Serotonin Concentration in Localized, Live Cell Nanoenvironments
}

\begin{abstract}
The mechanistic examination of an analyte concentration-dependent decrease in $\mathrm{CdSe} / \mathrm{ZnS}$ quantum dot (QD) fluorescence intensity in response to the neurotransmitter serotonin (5-hydroxytryptamine, 5-HT) in untargeted, ensemble-QD and receptortargeted, single-QD studies is presented. QD emission intensity loss by core and/or shell degradation, surface reconstruction, and energy transfer in the presence of 5-HT were examined and excluded as the origin of the 5-HT concentration-dependent QD luminescence loss. Time-resolved photoluminescence spectroscopy reveals that the QD lifetime decreases with increasing 5-HT concentration, which is indicative of a faster exciton decay pathway available to the QD via 5-HT. This is likely due to a complexation, followed by a photoinduced charge $\left(\mathrm{e}^{-} / \mathrm{h}^{+}\right)$transfer, between the QDs and 5-HT. Using single-particle cellular spectroscopy, it was verified that the rate of QD luminescence loss is proportional to the concentration of exogenous 5-HT at the singleQD level of a representative, complex environment.

This chapter is submitted for publication to Small as,

"Single CdSe/ZnS Quantum Dot Sensors for Quantification of Serotonin Concentration in Localized, Live Cell Nano-Environments"

Katye Fichter ${ }^{\dagger}, \underline{B e t h}$ A Manhat $^{\dagger}$, J. B. Alexander Ross, Tania Vu, and Andrea M. Goforth
\end{abstract}




\subsection{Introduction}

Direct bandgap II-VI semiconductor quantum dots (QDs) and core/shell QDs have received widespread attention for their wavelength-tunable visible light emission that is governed by a core-size-dependent quantum confinement (QC) effect. ${ }^{[3 a, 3 b, 7,16 e, 50]}$ The unique, favorable luminescence properties of QDs, e.g., broad absorption and narrow emission spectra, high photoluminescence quantum yields (QY), and resistance to photobleaching, have led to their successful employment in a variety of applications, including in light emitting diodes, ${ }^{[51]}$ and photovoltaics, ${ }^{[52]}$ and photodetectors. ${ }^{[53]}$ Also, because of these favorable emission properties, small sizes $(<25 \mathrm{~nm})$, relatively facile

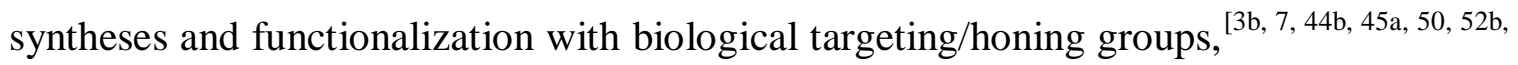
${ }^{54]}$ and long-term stability as compared to organic dyes, ${ }^{[16 e, 50,54 b, 54 c, 55]}$ the use of QDs in biomedical and biotechnological studies has sharply risen since the early 1990's. ${ }^{[7,54 e, 56]}$ Further increasing the utility of these fluorophores, Bruchez et al. spearheaded efforts to increase the biocompatibility of core/shell QDs (e.g., CdSe/ZnS QDs), where the inorganic shell is added to enhance the photophysical properties of the core, including the QY, by developing hydrophilic surface coatings amenable to a variety of chemical modifications. ${ }^{[16 e]}$ Since then, many studies have used bioconjugation techniques to target QDs to specific biological structures in live cells and tissues, dramatically increasing the utility of QDs as fluorescent nanoprobes to study bimolecular localization and tracking. ${ }^{[14,16 e, 44 b, 55 b, 57]}$

While QDs have been widely used as fluorescent biological tracking agents, ${ }^{[54 b, 58]}$ relatively few studies have taken advantage of their fluorescence property for sensing 
biologically important analytes in live subcellular environments. Early reports show that the intensity of ensemble-QDs (i.e., population) can correlate with changes in analyte concentration for the chemical sensing of biologically relevant ions (e.g., $\mathrm{K}^{+}, \mathrm{Cu}^{2+}$, $\left.\mathrm{Fe}^{3+}\right)^{[59]}$ and molecules, such as NO and sugars. ${ }^{[60]}$ Additionally, the intensity of ensemble-QDs can successfully detect biological analytes such as amino acids, ${ }^{[61]}$ proteins, ${ }^{[62]} \mathrm{DNA}^{[63]}$ and dopamine ${ }^{[45 \mathrm{~b}, 64]}$. However, these studies have been performed in controlled, in vitro environments. Moreover, while it has been shown that bioconjugated, targeted QDs may be individually resolved at the single-QD level, the use of single-QD nanosensors are sparse. ${ }^{[63 \mathrm{~b}, 65]}$ Finally, detailed investigations of sensing mechanisms at either the ensemble- or single-QD level have been given little attention, even though such investigations would offer greater understanding of how to tune QD nanosensors for analyte specificity.

Neurotransmitters, such as serotonin and dopamine, are released by neurons in discrete, modulated amounts, have been linked to biological functions, including mood and appetite control, as well as sleep modulation. Additionally, changes in neurotransmitter concentration have been found to underlie many different cognitive disorders $^{[66]}$ and psychological conditions. ${ }^{[67]}$ After release, neurotransmitters bind to receptors in small (10-100s $\mathrm{nm})$, localized compartments on neighboring neurons, and it is the neurotransmitter concentration in these regions that is of interest to measure. Current detection methods (e.g., in situ concentration determination via microprobe amperometry, or microdialysis with ex situ concentration determination) offer spatial resolution too low to measure neurotransmitter levels at the individual, localized sites of release and uptake. ${ }^{[67 \mathrm{~b}, 67 \mathrm{c}]}$ Therefore, targeted, high spatial resolution $(<50 \mathrm{~nm})$ sensors, 
including potential QD nanosensors, could contribute valuable information to the knowledge of the role of localized neurotransmitter imbalances in mental disorders and therapies. To this aim, the observation of an analyte concentration-dependent decrease of $\mathrm{CdSe} / \mathrm{ZnS} \mathrm{QD}$ fluorescence intensity in response to the neurotransmitter serotonin (5hydroxytryptamine, hereafter 5-HT) in both untargeted, ensemble-QD and receptortargeted, single-QD studies is presented. The possible origin of the 5-HT-accelerated QD emission quenching is investigated. Based on these results, a strategy for developing a family of targeted, neurotransmitter-specific single-QD nanosensors is presented.

\subsection{Experimental}

Materials: Laboratory prepared, PAA/TOPO-capped CdSe/ZnS QDs (QD 580 and $\mathrm{QD}_{593}$ samples, where TOPO = trioctylphosphine oxide, PAA $=$ (poly)acrylic acid, and $\mathrm{xxx}$ of the $\mathrm{QD}_{\mathrm{xxx}}$ designation indicates the emission maximum when the ensemble-QD solutions are excited) were a generous gift from Preston T. Snee, and their syntheses are described elsewhere. ${ }^{[68]}$ Briefly, the $\mathrm{CdSe} / \mathrm{ZnS}$ QDs were prepared from the high temperature pyrolysis injection of organometallic precursors, including, dimethylcadmium and trioctylphosphine selenide in TOPO. ${ }^{3}$ Diethylzinc and hexamethyldisilathiane were used as the $\mathrm{Zn}$ and $\mathrm{S}$ precursors to grow a $\mathrm{ZnS}$ shell on the CdSe QD. ${ }^{[69]}$ Commercially available PAA/TOPO-capped CdSe/ZnS QDs (QD 585 samples) were obtained from Life Technologies (Grand Island, NY). All ensemble-QD samples were stored in borate buffer $(\mathrm{pH}=8.3)$ under ambient atmosphere at $8{ }^{\circ} \mathrm{C} .5$-hydroxytryptamine hydrochloride (serotonin, 5-HT, $\mathrm{C}_{10} \mathrm{H}_{12} \mathrm{~N}_{2} \mathrm{O} \cdot \mathrm{HCl}$, Sigma-Aldrich) used as received. 
Transmission Electron Microscopy (TEM) and Dynamic Light Scattering (DLS): TEM and DLS were used to characterize the size and morphology of the CdSe/ZnS QDs. TEM was performed on an FEI Tecnai F-20 TEM operating at $200 \mathrm{kV}$. DLS measurements were performed on a Horiba LB-550 DLS. Measurements on five different CdSe/ZnS QDs concentrations were collected in order to determine a size distribution independent of concentration effects.

Ensemble UV-Visible absorption and Steady-State Photoluminescence (PL) Spectroscopy Measurements: All optical property measurements were carried out in borate buffer $(\mathrm{pH}$ = 8.3). UV-Visible absorption spectra $(190-700 \mathrm{~nm})$ for the neat QD or QD + 5-HT solutions were collected on a Shimadzu Model UV-2450 spectrophotometer. Steadystate PL spectra were collected on a Shimadzu-RF5301PC spectrophotometer (400-700 $\mathrm{nm})$ using an excitation wavelength of 340 or $370 \mathrm{~nm}$.

Observation of 5-HT-concentration-dependent QD Emission Intensity Loss for Ensemble$Q D_{580}, Q D_{585}$ and $Q D_{593}$ Samples: Individual QD solutions (2 $\mathrm{mL}, 10 \mathrm{nM}$ ) were prepared. Equal volume aliquots of borate buffer or $5-\mathrm{HT}(0.5 \mathrm{~mL}, 5-\mathrm{HT}$ concentrations ranging from $0.05-500 \mu \mathrm{M}$ ) were added to the QD solutions; the 5-HT additions were made immediately prior to UV-Vis and PL measurement.

\subsubsection{Mechanistic Determination}

Determination of $Q D$ Degradation (elemental loss and surface/other atomic rearrangements): Two QDs 585 solutions $(3 \mathrm{~mL}, 10 \mathrm{nM})$ were prepared, one neat and one in the presence of $1 \mu \mathrm{M}$ 5-HT. The samples were irradiated with a handheld UV lamp (6 
$\mathrm{W}, 115 \mathrm{~V}$, and $365 \mathrm{~nm}$ ) with PL spectra collected at 10 min intervals using $340 \mathrm{~nm}$ excitation. The solution was considered quenched when the emission maximum reached $<5 \%$ of its original value. Once quenched, the QD solutions were dialyzed against water using hydrophilic dialysis tubing for 3 hrs (Fisher Brand regenerated cellulose, MWCO $1000 \mathrm{Da})$. The retentates and dialysates were collected and dried to 2 and $10 \mathrm{~mL}$, respectively. They were submitted to Dr. Martine Ralle (Oregon Health \& Science University) for elemental analysis by inductively coupled plasma-mass spectroscopy (ICP-MS) on an Agilent Technologies 7700x series ICP-MS (with a Ni sampler and skimmer cones with $15 \mathrm{~L} / \mathrm{min}$ gas flow, $1.4 \mathrm{~mL} / \mathrm{min}$ carrier gas flow rate, and $1500 \mathrm{~W}$ rf power). Samples were diluted 10 -fold using $1 \%$ high purity $\mathrm{HNO}_{3}$ (Fisher, Trace Metal Grade) and ultrapure deionized, distilled water (Aries Vaponics). Cd, Se, Zn, and P calibration standards (VHG Labs, Manchester, NH) were prepared from a stock 1000 ppm solution. Sulfur ionizes in low quantities, and its mass is the same as $\mathrm{O}_{2}$; therefore, a better interference removal method would be required to determine $\mathrm{S}$ concentration.

Determination of Förster Resonance Energy Transfer (FRET) between the QDs and 5$H T$ : Absorption $(200-600 \mathrm{~nm})$ and emission $\left(\lambda_{\mathrm{ex}}=340 \mathrm{~nm}, \lambda_{\mathrm{ex}}=350-700 \mathrm{~nm}\right)$ spectra were collected on individual 5-HT $(100 \mu \mathrm{M})$ and QDs593 $(10 \mathrm{nM})$ solutions.

Determination of Photoinduced Charge Transfer (PCT) between QDs and 5-HT: Timeresolved photoluminescence (PL) spectroscopy measurements were collected on $\mathrm{QDs}_{585}$ $(2.0 \mathrm{~mL} 10 \mathrm{nM})$ in the presence of increasing concentrations of 5-HT $(0.5 \mathrm{~mL}, 0-1230$ $\mu \mathrm{M})$ under magic-angle polarization conditions by time-correlated single-photon counting (TCSPC) using a FLASC 1000 (Quantum Northwest, Liberty Lake, WA) 
sample chamber. A frequency-doubled, ps-Mira 900 Ti:Sapphire laser (Coherent, Santa Clara, CA) provided pulsed excitation at $375 \mathrm{~nm}$ and at a repetition rate of $4.7 \mathrm{MHz}$. The emission was isolated through a $25-\mathrm{nm}$ bandpass filter with peak transmission at $570 \mathrm{~nm}$ (Andover, Salem NH). The TCSPC data were collected at a timing resolution of 35 ps/channel using a Time Harp 200 PCI board (PicoQuant, Berlin), and each intensity decay curve was collected to $4 \times 10^{4}$ at the peak. An instrument response function was also collected to equivalent peak counts, using a light-scattering solution of dilute colloidal silica.

It was assumed that the intensity decay, $I(\mathrm{t})$, could be adequately described by sums of exponentials. In this case, $I(t)=\sum_{i=1}^{n} \alpha_{i} e^{-t / \tau_{i}}$, where $\tau_{i}$ is the lifetime and $\alpha_{i}$ is the amplitude of the $i^{\text {th }}$ component in the intensity decay. The intensity-weighted lifetime is then, $<\tau>=\sum_{i=1}^{n} \alpha_{i} \tau^{2}{ }_{i} / \sum_{i=1}^{n} \alpha_{i} \tau_{i}$. The data were fit by non-linear least-squares using iterative reconvolution (software package (FluoFit Pro V4.2.1 (PicoQuant, Berlin)), and the statistical goodness of the fits was assessed by the reduced $\chi^{2}$ value, randomness of the residuals, and autocorrelation of the residuals.

To calculate the error associated with the QD intensity-weighted lifetime values, Psi-Plot (Poly Software International, Pearl, NY) was used to fit the raw, unfit TCSPS data using a single exponential fit $\left(y=a e^{-b x}\right)$, where $a$ and $b$ are coefficients) to determine the standard deviation. 


\subsubsection{Single-QD Response to 5-HT}

Photophysical investigation of cell targeted, single-QD response to 5-HT in live cell nano-environments: Mouse neuroblastoma (N2a) cells, stably expressing HA-tagged 5HT1B (HA-5-HT1B) were cultured on glass cover slips. Cells were labeled with $\mathrm{QD}_{593^{-}}$ antibody conjugates to tether them to HA-5-HT1B receptors. These probes were conjugated using hydrazide-aldehyde (HA) linking as detailed elsewhere. ${ }^{[44 a]}$ Briefly, PEGylated QDs bearing terminal hydrazide moieties were reacted with oxidized anti-HA antibodies at a 1:1 stoichiometric ratio at a concentration of $300 \mathrm{nM}$ for $20 \mathrm{~min}$, and then diluted up to $2 \mathrm{~mL}$ with cellular media containing 10\% FBS and allowed to incubate for an additional 30 mins. This probe solution was added to the cells and allowed to incubate for 10 mins at room temperature; cells were then washed to remove excess (unbound) QDs. The cells were transferred to an imaging chamber containing Opti-MEM media (Life Technologies, Grand Island, NY) and imaged on an inverted epifluorescence Zeiss Axiovert 200m microscope using an ANDOR iXon CCD camera at a rate of 10 frames per second, collecting 500 frames for each condition. Media was supplemented with 5HT to bring the final concentration up the indicated value. To produce QD fluorescence traces, the average pixel intensity of single QD puncta was plotted against time.

\subsection{Results and Discussion}

The laboratory-prepared, 593 nm-emitting CdSe/ZnS QDs $\left(\mathrm{QD}_{593}\right)$ were found to have a core size of $4.1 \pm 1.1 \mathrm{~nm}$ via TEM (see Appendix). Because the CdSe cores were coated in a ZnS shell, stabilized with trioctylphosphine oxide (TOPO) and subsequently wrapped with polyacrylic acid (PAA, Figure 3.1), DLS was used to determine the 
effective size of the QDs. DLS measurements found that the QDs593 have a hydrodynamic diameter of about $9 \mathrm{~nm}$ (see Appendix).

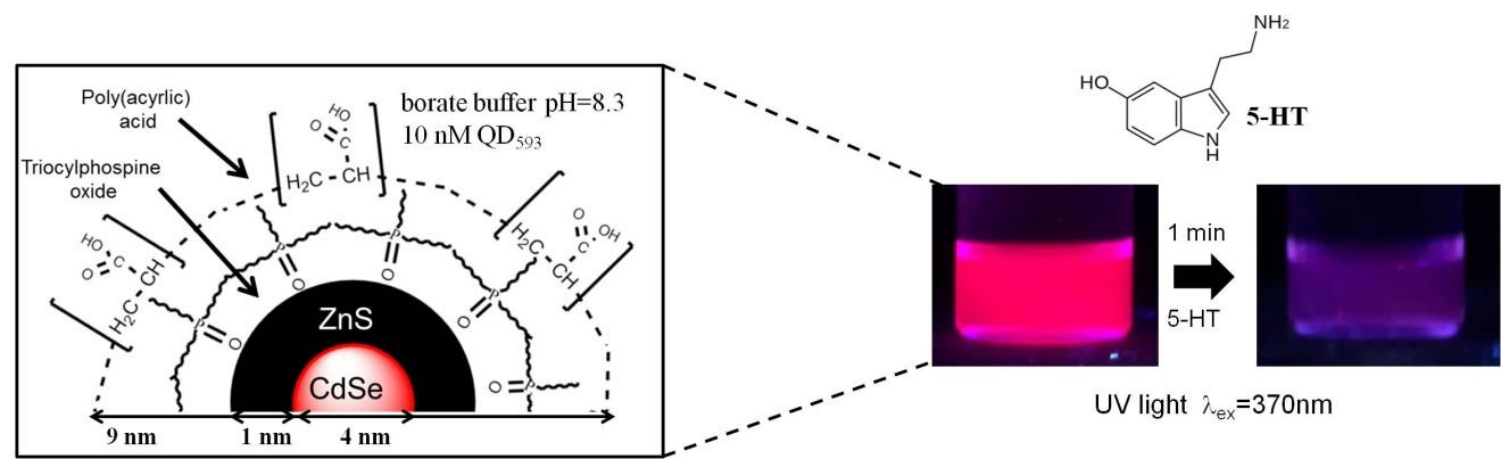

Figure 3.1. Scheme of red-emitting CdSe@ZnS QDs coated in TOPO and PAA with corresponding QD size; digital images of $10 \mathrm{nM}$ QDs before and after exposure to $100 \mu \mathrm{M}$ 5-HT.

\subsubsection{Photophysical Investigation of Ensemble-QD Response to 5-HT}

Upon the addition of 5-HT to a solution of red-emitting QDs 593 , a change in emission is visually observed within minutes (Figure 3.1). In ensemble (i.e., population) studies of $\mathrm{QD}_{593}(10 \mathrm{nM})$, aliquots of 5-HT $(0.5-500 \mu \mathrm{M})$ were added to stock solution of QDs593. The gross features of the QD absorption and emission spectra were preserved, and the observed emission intensity loss was greater with higher concentrations of added 5-HT (Figure 3.2). To examine the generality of this phenomenon, the same experiment was performed with commercially available $585 \mathrm{~nm}$ emitting and laboratory prepared 580 nm emitting CdSe/ZnS QDs (QD 585 and QD $_{580}$ samples, respectively, see Appendix). All batches of QDs were surface coated with TOPO and PAA, and all showed the same 5-HT concentration-dependent emission intensity loss trend. 


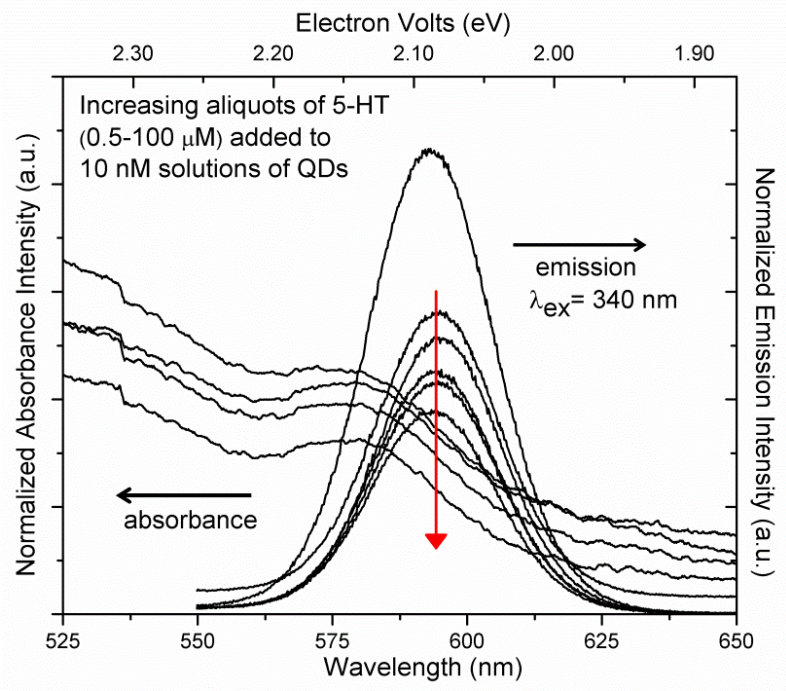

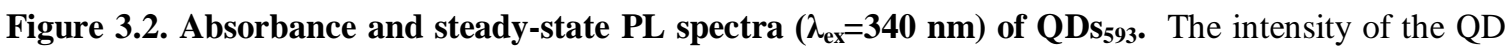
emission maximum at $593 \mathrm{~nm}(2.09 \mathrm{eV})$ was found to decrease when exposed to increasing concentrations of 5-HT. Commercially available $\left(\mathrm{QD}_{585}\right)$ and other laboratory prepared $\left(\mathrm{QD}_{580}\right)$ CdSe@ZnS QDs exhibited the same 5-HT concentration-dependant emission intensity loss (see Appendix).

\subsubsection{Mechanistic Determination of QD-5-HT Quenching}

Several mechanisms, alone or in combination, could be responsible for the observed, 5-HT-accelerated QD emission intensity loss, including: 1) degradation or other atomic rearrangements of the QD core, shell, and/or in the presence of the analyte; 2) resonant energy transfer from the QD to the analyte; 3) direct photoinduced charge transfer from the QD to the analyte; or 4) indirect photoinduced energy or charge transfer from the QD to the analyte, mediated by another matrix component (e.g., photogenerated singlet $\mathrm{O}_{2}$ ). It should be noted that the QDs also show emission intensity changes under prolonged UV irradiation in the absence of 5-HT; however, the rate of QD emission 
intensity loss when 5-HT is present is accelerated and proportional to the 5-HT concentration present.

Degradation of the QDs, or other 5-HT-accelerated atomic rearrangements have been observed by previous studies as being responsible for a QD emission intensity loss, particularly due to QD degradation arising from photo- or chemical-induced leaching of core or shell ions. ${ }^{[70]}$ This degradation increases the density of non-radiative exciton decay sites (i.e., defects), and can result in QD emission intensity loss. Furthermore, photo- or chemical-induced structural rearrangements, such as surface passivation and surface or interface reconstruction, are reported to result in variable emission intensity changes, including photoenhancement (emission intensity gain) or photodegradation (emission intensity loss). ${ }^{[64 b, 70 b, 71]}$

Other known types of analyte-dependent QD photodegradation have been modeled after molecular fluorophore photodegradation mechanisms, ${ }^{[72]}$ and are typically classified as Förster Resonance Energy Transfer (FRET) or Photoinduced Charge (e ${ }^{-}$or $\mathrm{h}^{+}$) Transfer (PCT) mechanisms. Because both FRET and PCT reactions provide alternate decay pathways for photogenerated QD excitons $\left(\mathrm{e}^{-} / \mathrm{h}^{+}\right.$pairs $)$, both would result in QD emission intensity loss due to decreased numbers of radiative recombination events from QD core states. ${ }^{[45 a, 60 e, 64 b, 73]}$

\subsubsection{Degradation of the QDs by Core/Shell Decomposition or other 5-HT- Aaccelerated Atomic Rearrangements}

The degradation (i.e., element loss) of the CdSe/ZnS QDs as a possible 
mechanism of the 5-HT-accelerated emission intensity loss was examined by subjecting, two identical, ensemble $\mathrm{QD}_{585}$ solutions to a prolonged light treatment; the experimental QD solution contained 1.0 $\mu \mathrm{M}$ 5-HT, while the control QD solution contained no 5-HT. Both QD solutions were irradiated using a handheld UV lamp until the emission intensity was $<5 \%$ of the original emission value, as determined by periodic spectroscopic measurements (Figure 3.3A and B). Following the light treatment, dialysis was used to separate intact particles (retentate) from ionic decomposition products $\left(\mathrm{Cd}^{2+}, \mathrm{Se}^{2-}, \mathrm{Zn}^{2+}\right.$, $\mathrm{S}^{2-}$, dialysate). ICP-MS was used to quantitatively evaluate the elemental compositions of the dialysates and the retentates of both the experimental and control reactions (See Appendix for full quantitative analysis results). While the $\mathrm{QD}_{585}+5-\mathrm{HT}$ solution lost emission intensity faster than the control QD 585 solution $(40 \mathrm{~min}$ vs. 70 min, respectively), neither dialysate contained a large amount of inorganic core ions $(0.68 \%$ and $0.59 \%$ of the total $\mathrm{Cd}^{2+}$ and $4.5 \%$ and $6.5 \%$ of the total $\mathrm{Se}^{2-}$ are found in the experimental and control dialysates, respectively, Figure 3.3C), indicating little QD core decomposition. Lack of QD core decomposition is also supported by the retention of the $585 \mathrm{~nm}$ emission maximum alongside the emission intensity loss. Degradation resulting in core element loss is expected to result in blue-shifted emission due to a shrinking core size and increasing bandgap, consistent with the expectations of QC; $;^{[2,70,71 b]}$ the emission spectra in Figure 3.3A and B (as well as Figure 3.2) indicate that the core size does not change. 

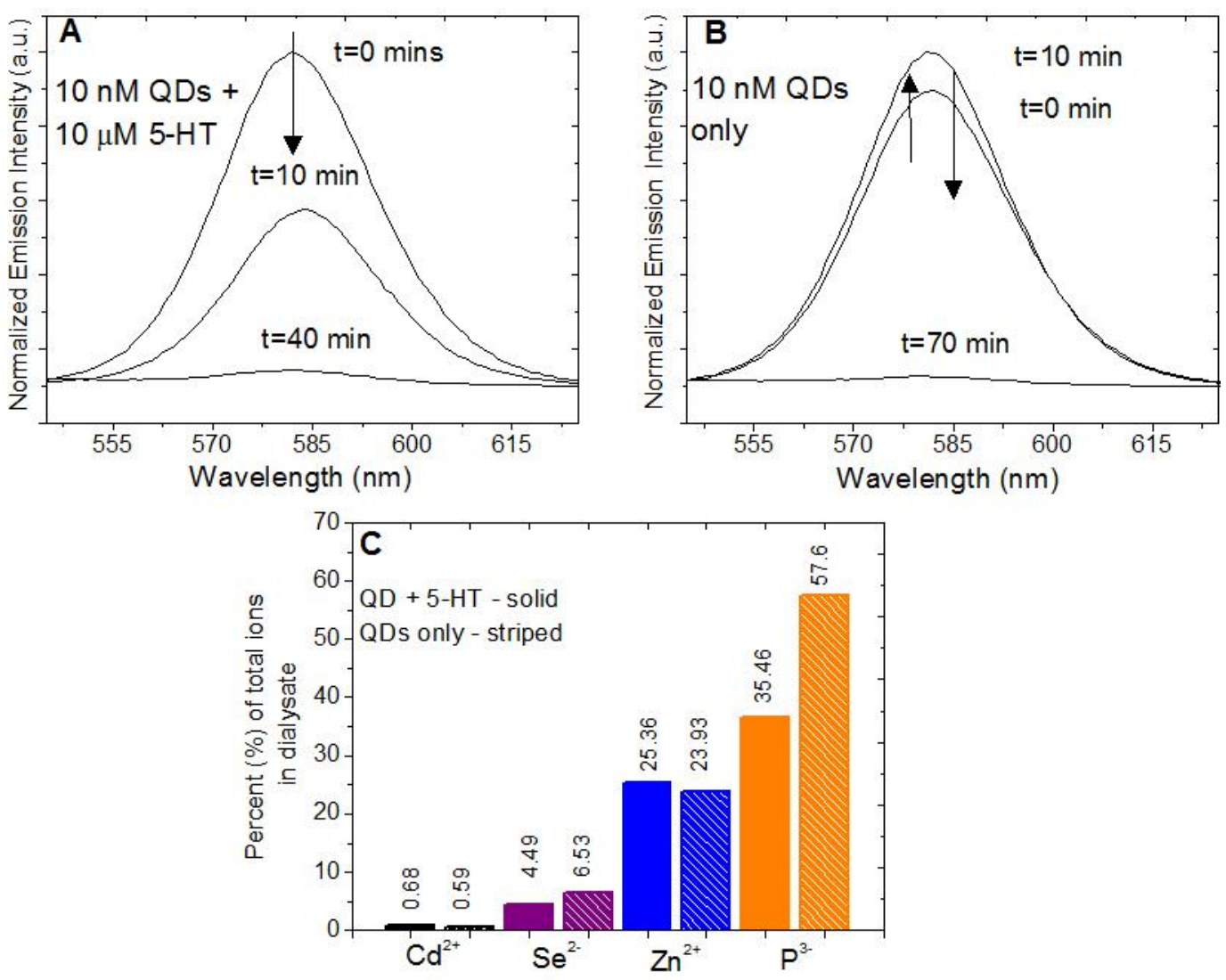

Figure 3.3. Emission spectra $\left(\lambda_{\mathrm{ex}}=\mathbf{3 4 0} \mathbf{~ n m}\right)$ and ICP-MS analysis. (A) Experimental $\mathrm{QD}_{585}+5-\mathrm{HT}$ and (B) control $\mathrm{QD}_{585}$ solutions collected during UV light treatment at the time points indicated (up to 70 mins); C. Percent of total $\mathrm{Cd}^{2+}, \mathrm{Se}^{2-}, \mathrm{Zn}^{2+}$, and $\mathrm{P}^{3-}$ determined by ICP-MS analysis for the experimental (solid bars) and control (striped) dialysates.

While core degradation was not indicated by the elemental analysis of the dialysates, ICP-MS results indicate that both QD solutions exhibit some shell decomposition under prolonged light exposure, which was independent of the presence or absence of 5-HT. The ICP-MS results showed that $25 \%$ and $24 \%$ of the total $\mathrm{Zn}^{2+}$ is found in the dialysates of the experimental and control samples, respectively. It should be noted that $\mathrm{S}^{2-}$ from the $\mathrm{ZnS}$ shell could not be discriminated by ICP-MS to provide 
information on shell decomposition, given its similarity in mass to atmospheric $\mathrm{O}_{2}$. Notably, however, there are no significant differences in the amounts of shell or core decomposition products between QD solutions with and without 5-HT; thus, shell or core degradation do not account for the accelerated loss of QD emission intensity in the presence of 5-HT.

The possible effects of surface or other structural rearrangements, as opposed to core/shell element loss, are difficult to assess with conventional chemical assays. Nonetheless, the degree of ligand passivation can be examined by ICP-MS as an indicator of surface rearrangements, where the observation of phosphorus $(\mathrm{P})$ from the stabilizing ligand, TOPO, in the dialysates after the photochemical reactions would indicate the relative extent of dynamic ligand exchange or the relative degree of surface ligand loss. In general, electrostatically associated, surface stabilizing ligands, such as TOPO, are known to undergo dynamic exchange in aqueous solutions. When they are surfacebound, stabilizing ligands are expected to passivate surface defect sites and to increase the emission intensity (photoenhancement), while removing the stabilizing ligands typically results in increasing the number of defect sites and lower emission intensity (photodegradation). ${ }^{[71 \mathrm{a}, 71 \mathrm{c}]}$ The ICP-MS results showed that a significant amount of phosphorus was found in the dialysates of both the experimental and control samples (37\% and $58 \%$ of the total phosphorus, respectively), indicating that the surface passivating ligand layer is changing under the experimental conditions, regardless of the presence or absence of the 5-HT analyte. Since the percentage of phosphorus in the control dialysate was greater than in the experimental dialysate, a greater number of non- 
radiative defects and lower emission intensity would be expected for the control sample if emission intensity loss were due to decreased ligand passivation alone. This expectation contradicts the emission observations since the emission intensity is lower for the 5-HT containing sample, which has lost less TOPO. Thus, it can be concluded that differences in the degree of surface passivation alone do not account for the 5-HT accelerated emission intensity loss. However, the data indicates that the competing effects of photodegradation and photoenhancement (observed in the $\mathrm{QD}_{585}$ solution, Figure 3.3B) due to decreased or increased ligand passivation, respectively, must be considered alongside other sources of emission intensity loss.

\subsubsection{Förster Resonance Energy Transfer (FRET) between the QDs and 5-HT}

For an excited QD to non-radiatively lose energy via FRET to a 5-HT molecule, resulting in QD emission intensity loss, spectroscopic overlap between 5-HT absorption and QD emission spectra is necessary. While colorless 5-HT does not seem to be a suitable FRET partner for red-emitting QDs, visible absorbance of 5-HT or 5-HTderivatives under certain conditions has been reported. ${ }^{[74]}$ However, under the experimental conditions used here $\left(\lambda_{\mathrm{ex}}=370 \mathrm{~nm}, \mathrm{pH}=8.3\right.$, $)$ no overlap between the 5-HT absorption and QD emission spectra is observed (Figure 3.4), making FRET an unlikely mechanism for the observed 5-HT concentration-dependent decrease in QD emission intensity. 


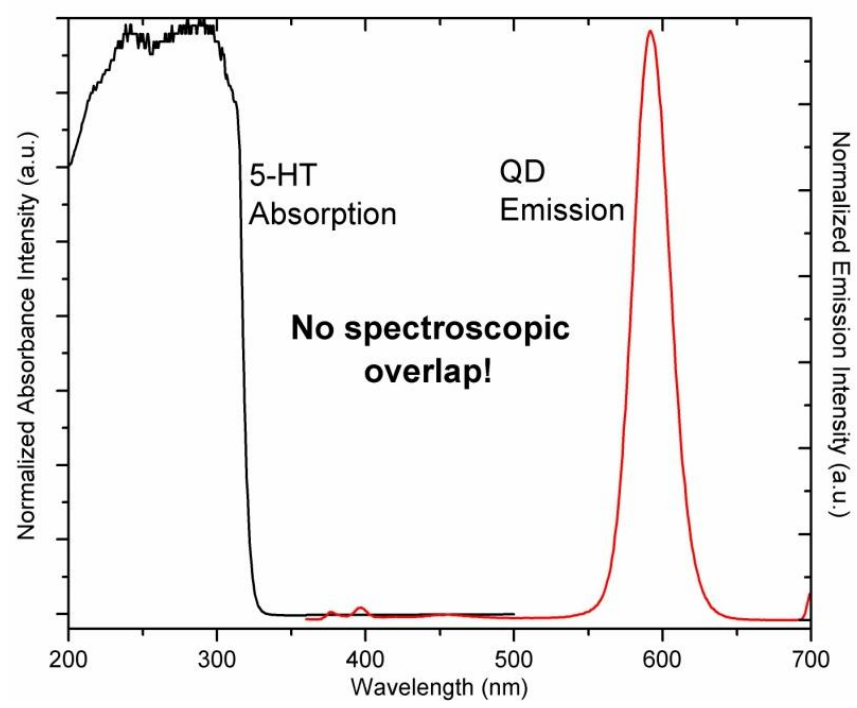

Figure 3.4. 5-HT absorption spectrum (200-600 nm) and $Q D_{593}$ emission spectrum $\left(\lambda_{\mathrm{ex}}=370 \mathrm{~nm}\right.$, $\lambda_{\mathrm{em}}=380-700 \mathrm{~nm}$ ) showing no spectral overlap.

\subsubsection{Photoinduced Charge $\left(\mathrm{e}^{-}\right.$or $\left.\mathrm{h}^{+}\right)$Transfer (PCT) between the QDs and 5-HT}

In considering a PCT reaction between the $\mathrm{CdSe} / \mathrm{ZnS}$ QDs and 5-HT, timeresolved PL spectroscopy was used to determine the temporal effects of the presence of 5-HT on the separation and recombination of QD excitons. A decrease in the average lifetime of the $\mathrm{CdSe} / \mathrm{ZnS}$ QD excited state indicates a competing excited state decay pathway that is faster than exciton recombination. If the decrease in the QD excited state lifetime in the presence of an analyte is proportional to the analyte concentration, then the alternate decay pathway should involve the analyte. 


\subsubsection{QD Excited State Lifetimes}

Using QDs593, time-resolved PL data was collected in the region of maximum QD emission intensity using excitation at $375 \mathrm{~nm}$ and a $570 \pm 25 \mathrm{~nm}$ band pass filter; the data was best-fit using a multi-exponential decay expression (see Experimental). The time-resolved emission decay for the neat $\mathrm{QD}_{593}$ solution is shown in Figure 3.5 (line a). Both the nanosecond timescale and the complex multi-exponential decay are typical for II-VI QDs and core/shell QDs. ${ }^{[45,75]}$ The multi-exponential decay is likely the result of heterogeneity in the excited state behavior of the QDs, the QD size distribution, or the distribution in the energy levels of trap sites. ${ }^{[75]}$ A best-fit using three exponentials was used to calculate an average intensity-weighted lifetime, $\tau_{\mathrm{o}}$, as given by Equation 3.1,

$$
<\tau>=\frac{\sum_{i=1}^{n} \alpha_{i} \tau^{2}{ }_{i}}{\sum_{i=1}^{n} \alpha_{i} \tau_{i}}
$$

The intensity-weighed lifetime of the neat $\mathrm{CdSe} / \mathrm{ZnS} \mathrm{QDs}_{593}$ was found to be $16.20 \pm$ 3.21 ns. Figure 3.5 also shows that the QD excited state decay is accelerated when the QDs are exposed to increasing concentrations of 5-HT; the average intensity-weighted lifetime of the QD emission decreases to as low as $13.25 \pm 3.31 \mathrm{~ns}$ in the presence of the highest measured concentration of 5-HT (1230 $\mu \mathrm{M}$, Table 3.1). 


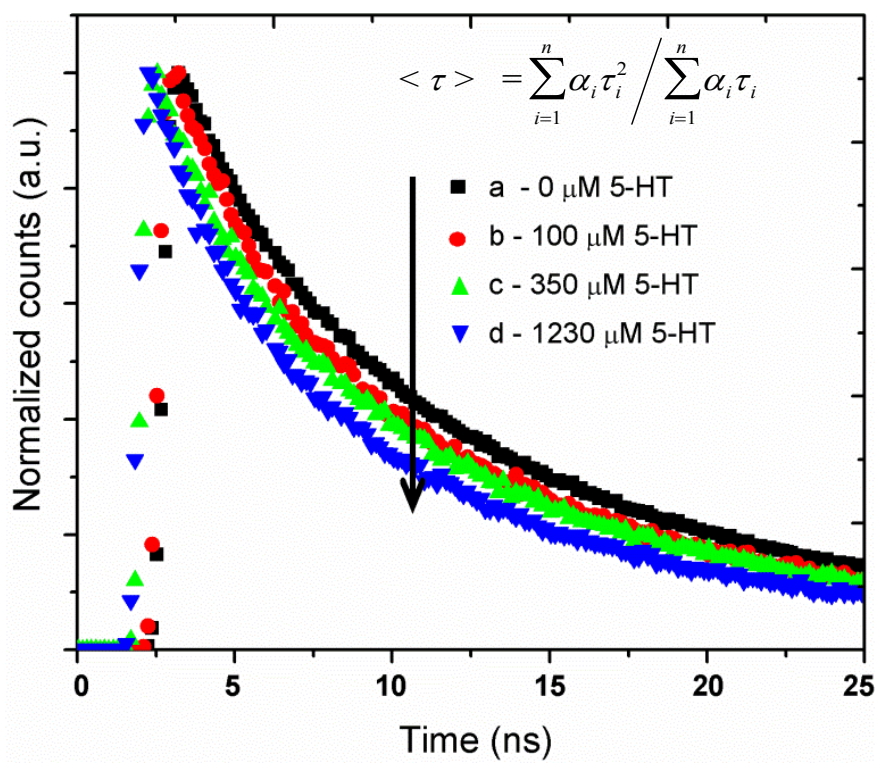

Figure 3.5. Excited state lifetime decays for $Q D_{593}$ solutions exposed to (a) $0 \mu \mathrm{M}$, (b) $100 \mu \mathrm{M}$, (c) 350 $\mu \mathrm{M}$, and (d) $1230 \mu \mathrm{M}$ 5-HT.

Table 3.1 Average Intensity-weighted lifetimes $(\tau)$ of $\mathrm{QDs}_{593}$ in the presence of 5-HT

\begin{tabular}{cccccccc}
\hline \multicolumn{1}{c}{ Average Intensity-weighted } & & & & & & \\
5 -HT, $\mu \mathrm{M}$ & lifetime, $\tau, \mathrm{ns}$ & $\tau_{1}, \mathrm{~ns}$ & $\alpha_{1}, \%$ & $\tau_{2}, \mathrm{~ns}$ & $\alpha_{2}, \%$ & $\tau_{3}, \mathrm{~ns}$ & $\alpha_{3}, \%$ \\
\hline 0 & $16.2 \pm 3.21$ & 5.32 & 0.24 & 15.5 & 0.712 & 72.5 & 0.0530 \\
100 & $15.1 \pm 3.00$ & 4.78 & 0.23 & 14.7 & 0.711 & 55.1 & 0.0630 \\
175 & $14.5 \pm 3.05$ & 13.1 & 0.71 & 4.06 & 0.180 & 37.2 & 0.114 \\
250 & $14.3 \pm 2.89$ & 4.54 & 0.24 & 14.2 & 0.701 & 51.1 & 0.0741 \\
350 & $13.9 \pm 1.01$ & 3.27 & 0.13 & 12.2 & 0.721 & 31.8 & 0.155 \\
550 & $13.5 \pm 2.61$ & 1.53 & 0.11 & 10.8 & 0.720 & 32.5 & 0.175 \\
1230 & $13.3 \pm 3.31$ & 11.9 & 0.70 & 3.16 & 0.175 & 32.5 & 0.142 \\
\hline
\end{tabular}

\subsubsection{Stern Volmer Analysis of the QD-5-HT Interaction}

The simplest kinetic model for solution-phase photodegradation is based on the statistical meeting of a fluorophore and analyte through diffusion, and is known as the 
Stern-Volmer (SV) model. This relationship explores the collisional quenching relationship between the fluorophore and analyte as given by Equation 3.2,

$$
\frac{\tau_{o}}{\tau}=1+k_{q} \tau_{0}[Q]
$$

where $\tau_{\mathrm{o}}$ and $\tau$ are the average QD lifetimes in the absence and presence of the quencher (5-HT), respectively, $k_{q}$ is the bimolecular quenching rate constant, and Q is the 5-HT concentration. $^{[72]}$ For a pure, diffusion-controlled collisional quenching, SV plots exhibit a linear relationship between $\tau_{\mathrm{o}} / \tau$ and the analyte concentration from which $k_{q}$ can be calculated. Shown in Figure 3.6, the SV plot of the lifetime ratios vs. 5-HT concentration yields a nonlinear relationship with a negative deviation at 5-HT concentrations $>500 \mu \mathrm{M}$. However, at the lowest measured 5-HT concentrations $(<350$ $\mathrm{nM})$, a linear relationship is observed between the ratio of the $\mathrm{QD}_{593}$ lifetimes and the 5HT concentration (inset Figure 3.6). From the slope of this plot, the quenching constant was determined to be $3.4 \times 10^{10} \mathrm{M}^{-1} \mathrm{~s}^{-1}$. The $k_{q}$ value, though on the expected order of magnitude for a simple diffusion process $\left(1 \times 10^{10} \mathrm{M}^{-1} \mathrm{~s}^{-1}\right),{ }^{[72]}$ is about 3 times faster than the expected $k_{q}$ value for a pure CT. 


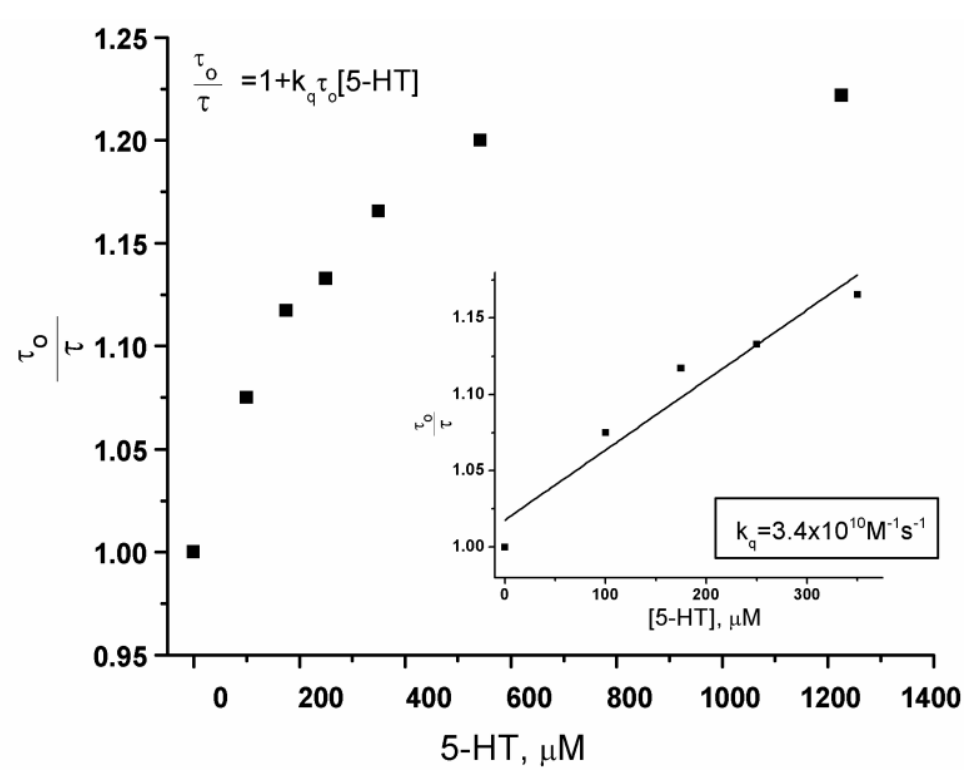

Figure 3.6. SV plot showing a non-linear relationship between the average intensity-weighted lifetime ratio, $\tau_{\mathrm{o}} / \tau$, of $\mathrm{QDs}_{593}$ and 5-HT concentrations up to $1230 \mu \mathrm{M}$; Inset SV plot illustrating the linear relationship between $\tau_{0} / \tau$ and 5 -HT at the lowest measured 5 -HT concentrations $(<350 \mathrm{~nm})$. The quenching constant, $\mathrm{k}_{\mathrm{q}}$, was found to be $3.4 \times 10^{10} \mathrm{M}^{-1} \mathrm{~s}^{-1}$.

The negative deviation from linearity observed in the SV plot of $\mathrm{QD}_{593}$ lifetime ratio versus 5-HT concentration has been observed in systems in which fluorescent amino $\operatorname{acids}^{[76],[77]}$ at the surfaces of proteins have been quenched by analytes, but that the amino acids buried within the protein are not. ${ }^{[7]}$ When considering QDs in the presence of 5HT, the negative deviation may indicate the differences in accessibility of CdSe@ZnS QD excitons or surface defect sites to the 5-HT molecules. ${ }^{[72,76 \mathrm{~b}, 77]}$ 
To analyze the QD-5-HT interaction further, a modified SV equation was used to account for the fraction of the QD potentially accessible to the 5-HT analyte $\left(f_{a}\right)$, given by Equation 3.3,

$$
\frac{\tau_{0}}{\Delta \tau}=\frac{1}{f_{a}}+\frac{1}{K_{a} f_{a}[Q]}
$$

where $K_{a}$ represents the SV quenching constant for that accessible portion. Both $f_{a}$ and $K_{a}$ are determined graphically. A plot of $\frac{\tau_{0}}{\Delta \tau}$ versus $\frac{1}{[5-\mathrm{HT}]}$ results in a y-intercept of $f_{a}^{-1}$ and a slope of $K_{a} f_{a}^{-1}$. From the modified SV equation, a linear relationship is observed (Figure 3.7) where $f_{a}$ and $K_{a}$ are found to be 0.23 and $4.66 \times 10^{3} \mathrm{M}^{-1}$, respectively. This derived value of $K_{a}$ (along with the average intensity-weighted lifetime of the QDs, 16.20 ns,) allows an estimated $k_{q}$ of $2.12 \times 10^{11} \mathrm{M}^{-1} \mathrm{~s}^{-1}$ to be calculated, which is two orders of magnitude greater than the expected constant for a pure collisional diffusion process. It is possible that a nondiffusion-based process is, at least in part, responsible for the QD-5HT interaction that results in the 5-HT concentration-dependent QD emission intensity loss, based on the overall high $k_{q}$ value. 


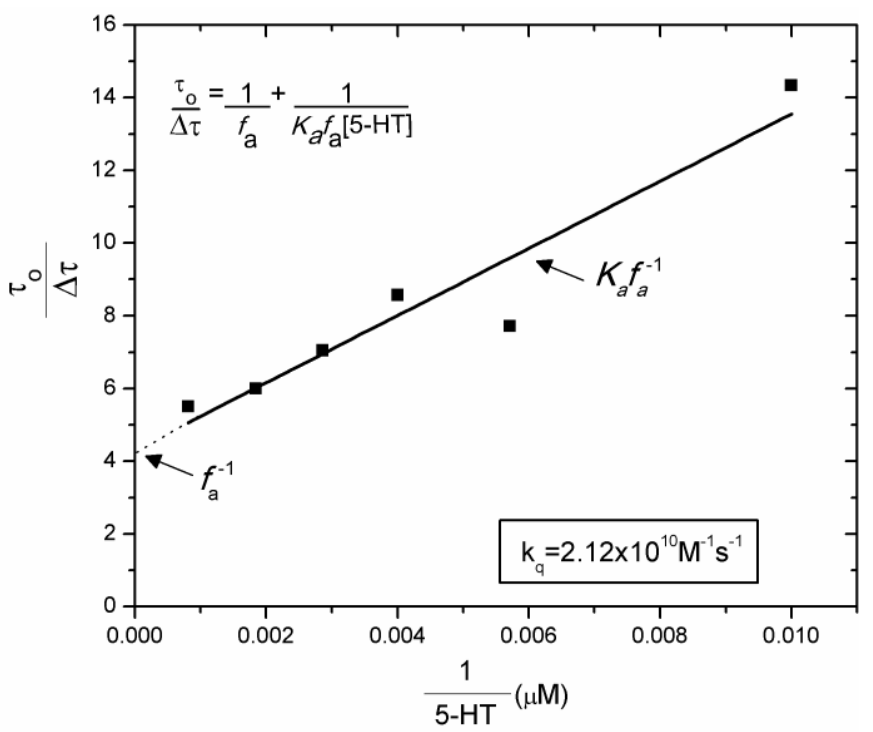

Figure 3.7. Modified SV plot illustrating the linear relationship 5-HT concentration and the average lifetime ratios of $\mathbf{Q D s}_{\mathbf{5 9 3}}$. A collisional constant, $k_{q}$ of $2.12 \times 10^{12} \mathrm{M}^{-1} \mathrm{~s}^{-1}$ was obtained from this plot.

\subsection{Quantitative Calibration of Single, 5-HT Receptor-Targeted QDs in Response to 5-HT Concentration in a Live Cell Environment}

To demonstrate the feasibility of using single QD nanosensors to detect biologically important analytes in subcellular environments, the fluorescence response of single $\mathrm{QDs}_{593}$ were measured and calibrated to 5-HT concentration. The conditions of the experiment were designed to mimic physiologically-relevant conditions by 1) setting the 5-HT concentration to a physiological range (1-20 nM extracellular concentration, as measured by microdialysis experiments ${ }^{[78]}$, and 2) measuring the concentration of 5-HT in the vicinity of single 5-HT receptors by recording emission intensity from single QD nanosensors that are specifically attached to 5-HT receptors in live cells. The 5-HT receptor, subtype 1B, was a target for QD labeling because of its implication in a variety of physiological disorders. ${ }^{[79]}$ Targeting of the QD nanosensor to 5-HT1B receptors was 
achieved at a high level of specificity using a previously developed methodology (See Experimental). ${ }^{[80]}$ Briefly, the gene for 5 -HT1B was modified by adding an HA tag to the extracellular N-terminus end. N2a cell lines stably expressing HA-5-HT1B were isolated and cultured, and single or very small groups of 5-HT1B receptors on these cells were labeled with anti-HA/QD conjugates. Live, $\mathrm{QD}_{593}$-labeled cells (Figure 3.8A) were then exposed to varying 5-HT concentration in the $\mathrm{nM}$ range. Very fast (10 frames per second) time-lapse movies of single QDs on these live cells were captured, and traces of the average single-QD pixel intensities were created by plotting average pixel intensity of QDs puncta vs. time (Figure 3.8B).
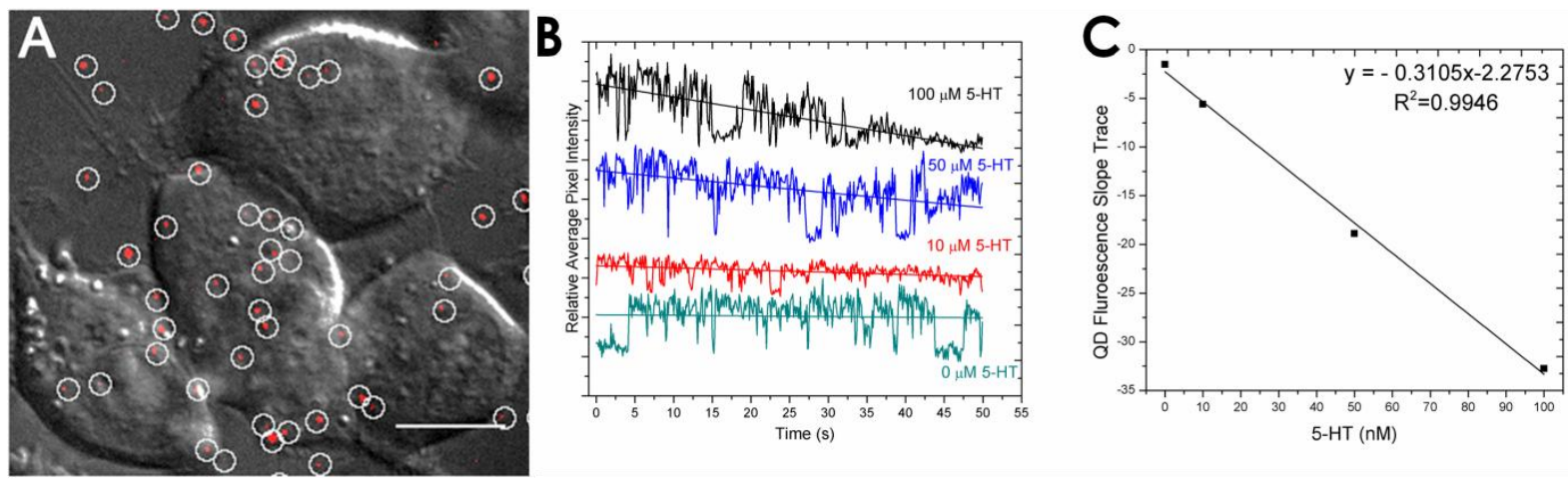

Figure 3.8. Single QD nanosensors, targeted to 5-HT receptors in a live cell environment quantitatively calibrated to 5-HT concentration down to $10 \mathrm{nM}$. A. Single $\mathrm{QDs}_{593}$, circled in white, were targeted toward 5-HT1B receptor in live N2a cells, scale bar $=10 \mu \mathrm{M}$; B. Offset pixel intensity traces of single QDs. The slopes of these traces become more negative with increasing 5-HT concentration; C. Plot depicting the linear relationship between 5-HT concentration and the slope of QD pixel intensity traces.

Traces of the average pixel intensity of single QDs exhibited a negative slope for all 5-HT concentrations tested (Figure 3.8B). These data demonstrate that QD 
nanosensors have the sensitivity to respond to 5-HT at concentrations as little as $10 \mathrm{nM}$, which is a physiologically relevant concentration in extracellular spaces in vivo in the brain. $^{[78 b, 78 c, 81]}$ However, it is likely that the concentration of 5-HT closer to the sites of 5HT signaling, such as neural synapses, may be much more concentrated. Furthermore, the slopes of QD traces decrease with increasing 5-HT concentration in a linear manner. This trend is depicted in a calibration plot (Figure 3.8C), which plots 5-HT concentration vs. the slope of QD pixel intensity traces generated in Figure 3.8B. This plot reveals a linear relationship in the range of $0-100 \mathrm{nM} 5-\mathrm{HT}$, demonstrating that single-QD nanosensor response may be calibrated to 5-HT concentration even in a complex, livecell environment. These experiments demonstrate the feasibility of using QD nanosensors to quantify 5-HT concentration in a complex, live-cell environment at biologically relevant concentrations and show promise for revolutionizing the knowledge of neurotransmission at the single-receptor level.

\subsection{Discussion on QD-5-HT Quenching Mechanism and Development of}

\section{Neurotransmitter-Specific Nanosensors}

Equilibrium-mediated, QD-surface complexation models have been previously

proposed for both 5-HT- and dopamine (DA)-conjugated CdSe/ZnS QDs. ${ }^{[45 b, 62 a, 64 b, 82]}$ These reports have shown that this conjugation appears to lead to a decrease in QD emission intensity, ${ }^{[62 a, 82-83]}$ which has been attributed to CTs between the conjugated neurotransmitter and the QD host. In our system, the temporal changes in QD lifetimes in the presence of 5-HT, the increased quenching constant, and the net loss of TOPO ligands from the QD surface supports the dynamic equilibrium of TOPO ligands leaving 
open QD surface sites that may be subsequently occupied by 5-HT. Thus, at higher concentrations of 5-HT, a greater number of QD-bound 5-HT molecules would be expected for a more facile $\mathrm{CT}$, which supports the calculation of a $k_{q}$ value that is larger than that of a pure diffusional quenching process. However, the experimental dialysate from the QD+5-HT photochemical reaction has less phosphorous than the corresponding control dialysate (QDs only). This is consistent with 5-HT having a similar, but lower, binding affinity for the QD surface relative to TOPO. While 5-HT is able to occupy some of the surface sites, the competition between TOPO and 5-HT causes a shift of the complexation equilibrium toward a higher overall degree of ligand surface coverage, and furthermore, towards an increased surface coverage by the stronger binding ligand (TOPO) (Figure 3.9). Together, the collective data support the hypothesis that a faster, alternate decay pathway for the QD excitons exists via 5-HT and that a PCT reaction between the CdSe/ZnS QDs and 5-HT molecules, some of which are surface-bound, is the likely mechanism of QD emission intensity loss. ${ }^{[73]}$ 

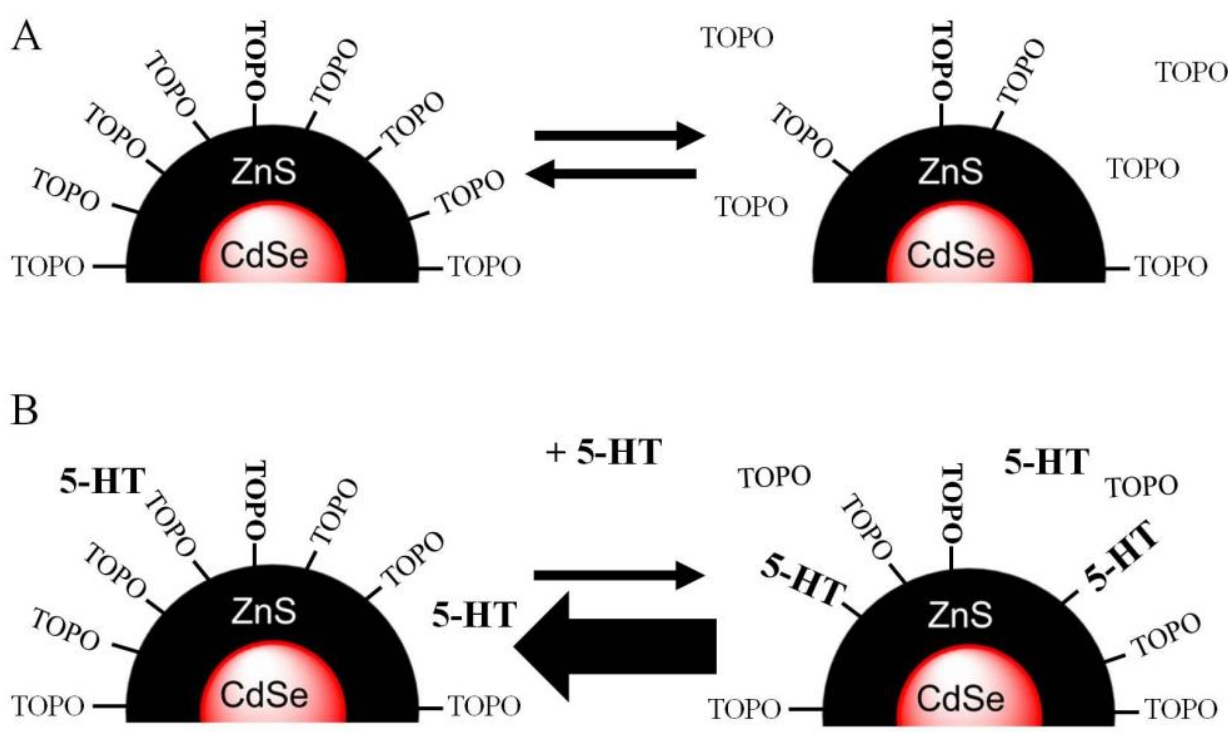

Figure 3.9. Schematic of CdSe/ZnS QDs undergoing dynamic loss/gain of surface ligands. (A). In the presence of 5-HT, more TOPO remains on the QD surface. While 5-HT can bind to the QD surface, its affinity is likely less than that of TOPO.

Solution, ensemble-QD data indicate that the 5-HT concentration-dependent PCT is the major mechanism of QD emission intensity loss. Not only is the excited state decay lifetime of the QD exciton well-correlated with 5-HT concentration in ensemble, solution QD studies, but also the rate of emission intensity loss is well-correlated with 5HT concentration in receptor targeted, single QD studies. Additionally, other known sources of QD emission intensity loss, both environmental and analyte-dependent, have been examined and eliminated. Although dynamic ligand exchange or ligand loss and QD shell element loss under prolonged photoexcitation contribute to gradual QD emission intensity changes over long time periods (hours), 5-HT additions result in more dramatic emission intensity changes over shorter periods of time (minutes), and the rate of emission intensity loss is increased with increased 5-HT concentration. 
While the parallel observations that the rate of intensity loss is correlated with 5HT concentration for both ensemble and single QDs is promising for translating the QD5-HT nanosensors construct to clinical applications for localized 5-HT measurement, charge transfer from QDs to other species whose reduction potentials are similar to 5-HT, are also possible results in poor sensor specificity. For example, previous investigators have reported that group II-VI core/shell QD fluorescence changes are the result of the production of reactive oxygen species (ROS, e.g., singlet oxygen), particularly of reduced dioxygen species, such as superoxide, peroxide, hydroxide. ${ }^{[84]}$ While the conduction band of quantum-confined $\mathrm{CdSe}^{[64 \mathrm{~b}, 85]}$ does not allow for the direct reduction of molecular oxygen, it has also been speculated that the surface ligands or $\mathrm{ZnS}$ shell degradation may promote the reduction of dioxygen. ${ }^{[64 \mathrm{~b}, 70,71 \mathrm{~b}, 85]}$ If this is the case, then the production of reduced $\mathrm{O}_{2}$ species via core/shell QDs would result in a decreased QD emission intensity that is not solely due to the 5-HT analyte. To examine the effects of dioxygen on the 5-HT accelerated QD emission intensity loss, control experiments were conducted in the presence and absence of dioxygen, where aqueous samples were sparged with argon prior to 5-HT addition and photochemical treatment. Importantly, the rate of $\mathrm{QD}$ emission intensity changes remained the same in $\mathrm{O}_{2}$ or $\mathrm{N}_{2}$ atmospheres, regardless of the presence or absence of 5-HT (See Appendix). Therefore, it is unlikely that the observed emission loss of the QDs is due to interactions with molecular oxygen. Also, while other reaction factors may contribute to the gradual emission intensity loss, this experiment supported the involvement of 5-HT in the dramatic emission intensity loss. 
Although other potential exogenous or endogenous interferents to 5-HT sensing in the biochemical environment may exist, it is known that PCTs can be tuned for specificity by appropriately matching the electrochemical potentials of charge donors and acceptors. The relative energetic positions of QD valance and conduction bands (e.g., versus NHE) may be tuned synthetically to achieve specificity, by utilizing a QD of appropriate composition and size, where both factors can control the band edge energies of the QDs.. ${ }^{[56,86]}$ For example, a QD-neurotransmitter sensor may be made specific ${ }^{[87]}$ for 5-HT versus an endogenous competitor, CA, through band gap tuning, where the 5HT acceptor level is at an energy within the QD bandgap and the DA acceptor level ${ }^{[88]}$ is at an energy level outside the bandgap of the QD.

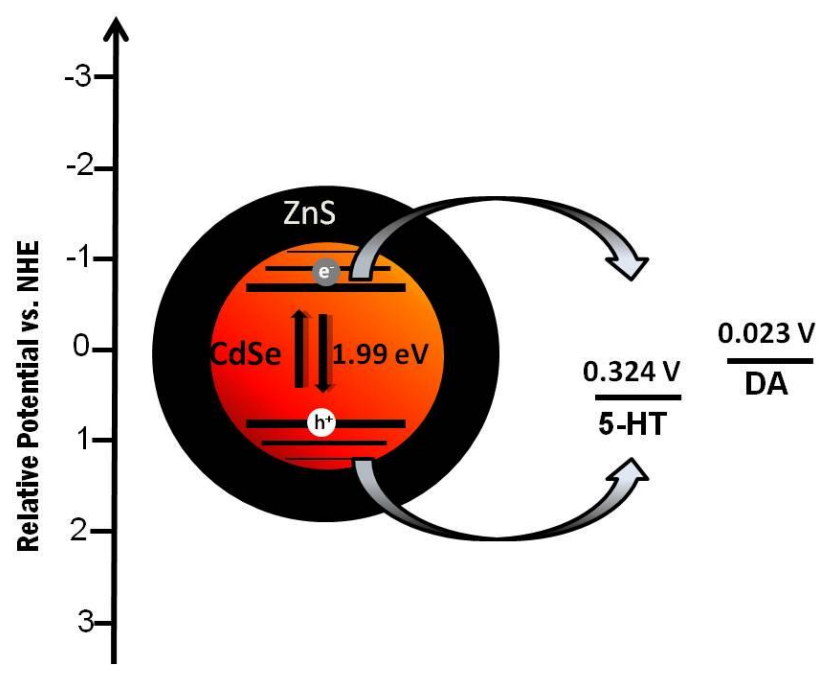

Figure 3.10. Relative energy levels of red-emitting CdSe@ZnS QD valance bands and conduction bands for nanosensors relative to possible analytes, including 5-HT and DA. 


\subsection{Conclusion}

Red emitting CdSe/ZnS quantum dots (QDs) exhibit a concentration-dependent emission decrease when exposed to the neurotransmitter serotonin (5-hydroxytryptamine, 5-HT) in both untargeted, ensemble-QD and receptor-targeted, single-QD studies. A mechanistic study to better understand the 5-HT-QD interaction was performed and ensemble-QD solutions were found not to undergo photoinduced core/shell degradation or energy transfers when exposed to 5-HT. Time-resolved spectroscopy has shown that the QDs exhibit a decrease in the emission lifetime with increasing 5-HT concentration, indicative of a faster exciton decay pathway via 5-HT that likely results from a photoinduced charge $\left(\mathrm{e}^{-} / \mathrm{h}^{+}\right)$transfer $(\mathrm{PCT})$. Based on the dynamic ligand loss and gain of electrostatically bound TOPO from the QD surface, in addition to the high quenching constant, it is likely that the PCT interaction occurs from the complexation of vacant QD surface sites and 5-HT molecules. Molecular oxygen and oxygen-centered species have been excluded as potential mediators to the PCT reaction. While other mediators may exist, these interactions with QDs may be avoided based on the ability to tailor the QD bandgaps by size and composition to the redox potential of the sensing analyte. Using single-particle spectroscopy, the rate of QD luminescence loss is proportional to the concentration of 5-HT in the biologically relevant nM range. With the nanometer spatial resolution afforded by fluorescent QD sensors, as well as the ability to selectively place them in biologically-active subcellular locations, nanosensors offer the promise of quantitative, selective sensing at biologically relevant, spatially-confined sites that are not currently possible, such as at specific sites of neurotransmitter release and uptake. 
Synthesis of Functionalized Si Nanoparticles using a Two-Step Metathesis and Hydrosilylation Reaction

\begin{abstract}
Emission from Si nanoparticles (NPs) is affected by both NP size and NP surface chemistry. To examine this, silicon nanoparticles (Si NPs; $5.02 \pm 1.21 \mathrm{~nm}$ ) were synthesized using a solution based Zintl salt metathesis between sodium silicide, $\mathrm{NaSi}$, and ammonium bromide, $\mathrm{NH}_{4} \mathrm{Br}$. In the presence of the coordinating solvent, N,N'dimethylformamide (DMF), this reaction resulted in blue emitting Si NPs with partial hydride-termination. Using thermal hydrosilylation (HS) reactions with 1-decene in DMF, hydrophobic Si NPs were obtained, which showed evidence of Si-C termination. However, the HS reaction with 1-decene simultaneously produced hydrophilic Si NPs that showed evidence of coordination and termination with the solvent, DMF. In efforts to avoid the surface competition between the solvent and ligand, two approaches for the HS with 1-decene were taken: 1) HS reaction with 1-decene using a chemical initiator, rather than heat, and 2) HS reaction with 1-decene in a non-coordinating solvent. While these reactions did produce the desired fluorescent hydrophobic Si NPs, the FT-IR intensity of the covalent $\mathrm{Si}-\mathrm{C}$ stretches, as well as the emission intensities, suggests that these reactions produced fewer decene-functionalized $\mathrm{Si}$ NPs. Together these experiments suggest that a coordinating solvent is necessary to produce a high number of NPs using the Zintl salt metathesis or to stabilize the NP during the HS reactions.
\end{abstract}




\subsection{Introduction}

Visible photoluminescence (PL) from porous silicon ${ }^{[18 \mathrm{a}]}(\mathrm{Si})$ was discovered in the early 1990s, and since then, Si has been studied as a potential light emitter, even though the origin of the optical properties from Si have been debated. ${ }^{[6 \mathrm{~d}, 90]}$ Nonetheless, luminescent, freestanding Si nanoparticles (NPs) have been attractive for many applications, including those in biomedicine, such as imaging and sensing agents. ${ }^{[6 \mathrm{~d}]}$ Additionally, $\mathrm{Si}$ is the foremost technologically relevant element for electronic applications. ${ }^{[1]}$ Therefore, careful development of Si-based fluorophores with controlled optical and chemical properties could lead to increased applications of luminescent $\mathrm{Si}$ nanostructures in Si-based opto-electronics.

Like direct bandgap quantum dots $(\mathrm{QDs})^{[3 \mathrm{~b}, 3 \mathrm{~d}, 54 \mathrm{~b}]}$ (i.e., CdSe and $\mathrm{PbS} \mathrm{QDs}$ ), indirect bandgap Si NPs can exhibit size-dependent optical properties consistent with quantum confinement ${ }^{[92]}(\mathrm{QC})$. QC affects the physical properties of semiconductors as they decrease from the bulk to the nanosized regime. Removing atoms from bulk semiconductor gives rise to NPs with a size influence on the magnitude of the bandgap; this gives semiconductor NPs size-tunable optical properties such that smaller NPs have a more blue shifted emission. ${ }^{[2,92 a]}$ Si NPs, approximately less than $5 \mathrm{~nm}$, are expected to be affected by QC and to possess these size-tunable optical properties. Therefore, in order to realize the successful application of Si NPs in either optoelectronic or biological applications, monodisperse $\mathrm{Si}$ NPs in the 1-5 $\mathrm{nm}$ range must be developed.

Several published methods have been reported to produce small, emissive Si NPs, including high-temperature (solid state) and low-temperature (solution) methods, 
electrochemical methods, plasma methods, and deposition techniques. ${ }^{[3 c, 19,}$ 83, 93] However, Si NPs produced by low-temperature solution methods have generated the most variety in the surface termination of Si NPs. The conventional Zintl metathesis reaction between metal silicides and ammonium halide solids, for example, has been used to generate Si NPs in a variety of solvents, including N,N-dimethylformamide (DMF), dioctylether (DOE), and dimethoxyethane (DME). ${ }^{[20 \mathrm{~d}, 94]}$ These reports have indicated that at least some of the Si NP surfaces bear reactive hydride-termination, and that these NPs may undergo subsequent surface manipulations of the $\mathrm{Si}-\mathrm{H}$ bonds, ${ }^{[95]}$ including hydrosilylation (HS) reactions initiated by heat, UV light, or chemical catalyst. HS reactions impart the NPs with stable, covalent Si-C linkages with alkyl, carboxylic acid, or amine surface terminating groups. ${ }^{[20 a, 20 b, 96]}$

The emission from Si NPs is also affected by the surrounding environment, including surface functionalization and solvents. ${ }^{\text {90a, 97] }}$ Therefore, functionalized Si NPs are often obtained from multi-step reactions, which allow the core and surface termination steps to be completed independently. Preparing the core and performing the surface manipulations in individual steps prevents unintended surface modifications of the Si NP surfaces, which can further complicate discerning the effects of NP size vs. surface chemistry on the origin Si NP emission. Herein, the synthesis of alkenefunctionalized Si NPs by a two-step Zintl metathesis and HS reaction is presented. While using a thermal-initialized HS reaction with 1-decene, the coordinating solvent, DMF, was found to compete with 1-decene for surface sites on the Si NP. This caused the fluorescent, hydrophilic Si NPs to be obtained alongside the desired fluorescent, 
hydrophobic decene-functionalized Si NPs. To minimize the solvent-ligand competition, the HS reaction with 1-decene was performed, 1) using a chemical catalyst rather than heat to initiate the HS in the presence of DMF, and 2) using thermal HS in the presence of a non-coordinating solvent with a comparable boiling point. These reactions were aimed at preventing solvent-ligand competition for the Si NP surface, which should enhance the capping efficiency by 1-decene.

\subsection{Si NP Experimental and Characterization}

All synthetic manipulations were performed either in a $\mathrm{N}_{2}$-filled glovebox or under an inert (Ar) atmosphere using standard Schlenk techniques. All aqueous solutions were prepared using electrophoretically pure water $\left(\mathrm{nH}_{2} \mathrm{O}, 18 \mathrm{MOhm} \bullet \mathrm{cm}\right.$ resistivity). Prior to use, sodium silicide (NaSi, $\mathrm{SigNa}, 80 \%$ ) was ball milled for 15 mins under $\mathrm{N}_{2}$ in a tungsten carbide-lined milling vial with two- $1 \mathrm{~cm}$ tungsten carbide balls using a high energy mill mixer (Spex $8000 \mathrm{M})$. Ammonium bromide $\left(\mathrm{NH}_{4} \mathrm{Br}\right.$, Aldrich, $\left.>99.99 \%\right)$ was dried in vaccuo at $100{ }^{\circ} \mathrm{C}$ before use. N,N-Dimethylformamide $\left(\mathrm{CHON}\left(\mathrm{CH}_{3}\right)_{2}\right.$, Sigma Aldrich, > 99.8\%) and o-xylene $\left(\mathrm{C}_{6} \mathrm{H}_{4}\left(\mathrm{CH}_{3}\right)_{2}\right.$, Sigma Aldrich, $\left.\geq 98 \%\right)$ were dried over

molecular sieves ( $3 \AA$ ) prior to use. Potassium hexachloroplatinate (IV) $\left(\mathrm{K}_{2} \mathrm{PtCl}_{6}\right.$, Sigma Aldrich, 98\%) and 1-decene $\left(\mathrm{C}_{10} \mathrm{H}_{20}\right.$, Sigma Aldrich, $\left.98 \%\right)$ were used as received.

\subsubsection{Si NP Synthesis}

N,N-Dimethylformamide (DMF)-synthesized Si NPs: $\mathrm{NaSi}(9.8 \mathrm{mmol})$ and $\mathrm{NH}_{4} \mathrm{Br}$ (19 mmol) were loaded into to a three neck, round-bottomed flask inside an $\mathrm{N}_{2}$-filled glovebox. The closed reaction vessel was then transferred to the Schlenk line, and $50 \mathrm{~mL}$ 
of DMF was added via cannula. A black suspension formed, which was stirred under reflux $\left(155^{\circ} \mathrm{C}\right)$ overnight. After cooling to room temperature, the black solids were removed by air-free vacuum filtration, leaving a yellow-colored solution containing the DMF-synthesized Si NPs. Neat DMF was removed by rotary evaporation.

o-Xylene (xylene)-synthesized Si NPs: $\mathrm{NaSi}(5 \mathrm{mmol})$ and $\mathrm{NH}_{4} \mathrm{Br}(10 \mathrm{mmol})$ were loaded into to a three neck, round-bottomed flask inside an $\mathrm{N}_{2}$-filled glovebox. The closed reaction vessel was then transferred to the Schlenk line, and $25 \mathrm{~mL}$ of xylene was added via cannula. A black suspension formed, which was stirred under refluxed (144 ${ }^{\circ} \mathrm{C}$ ) overnight. After cooling to room temperature, the black solids were removed by airfree vacuum filtration, leaving a colorless to pale yellow-colored solution containing the xylene-synthesized Si NPs. Neat xylene was removed by rotary evaporation.

\subsubsection{Hydrosilylation (HS) of the prepared Si NPs}

Heat-catalyzed HS using 1-decene: The precursor Si NPs (from the synthesis in DMF or xylene) were brought up in $25 \mathrm{~mL}$ of respective solvent in a two neck, roundbottom flask. While stirring, $2 \mathrm{~mL}$ of 1-decene was added via syringe and the solution was refluxed for $12 \mathrm{hrs}$. Subsequently, a water/hexanes extraction was used to separate the Si NPs into hydrophobic and hydrophilic fractions. Both layers were rota-vapped to dryness and re-dispersed in $5 \mathrm{~mL}$ of the same solvent (water or hexanes).

Chemically-catalyzed HS using 1-decene: The DMF-synthesized Si NPs were brought up in $25 \mathrm{~mL}$ of DMF containing $0.7 \mathrm{~g}$ of the catalyst, $\mathrm{K}_{2} \mathrm{PtCl}_{6}$, in a two neck round-bottom flask. While stirring, $2 \mathrm{~mL}$ of 1-decene was added via syringe, and the solution was stirred for $12 \mathrm{hrs}$. A water/hexanes extraction was used to separate the $\mathrm{Si}$ 
NPs into hydrophobic and hydrophilic fractions. Both layers were rota-vapped to dryness and re-dispersed in $5 \mathrm{~mL}$ of the same solvent (water or hexanes).

\subsubsection{Si NP Characterization}

Transmission Electron Microscopy (TEM): TEM, performed on a Tecnai F-20 HR-TEM operating at $200 \mathrm{kV}$, was used to assess the morphology, size, size distribution, and composition of the as-prepared and functionalized Si NP samples. Samples were prepared by drop casting diluted aliquots of Si NPs onto 400-mesh holey carbon-coated copper grids (SPI, West Chester, Pa), and dried at $100^{\circ} \mathrm{C}$ overnight.

Fourier Transform Infrared (FT-IR) Spectroscopy: FT-IR spectra of the neat solvents, neat ligands, as-prepared Si NPs, and functionalized Si NPs were collected on a Nicolet iS10 spectrometer using a diamond ATR attachment. NP solutions were drop cast onto the ATR crystal and the solvent was evaporated as necessary using a heat gun to deposit a film.

UV-Visible and Standard Photoluminescence (PL) Spectrophotometry: UV-Vis absorption spectra were collected on a Shimadzu UV-2450 UV-Vis spectrophotometer in a standard 1-cm quartz cuvette. Standard PL measurements were collected on a Shimadzu-RF5301PC spectrophotometer using various excitation wavelengths.

\subsection{Results and Discussion}

The Zintl metathesis reaction between sodium silicide, $\mathrm{NaSi}$, and ammonium bromide, $\mathrm{NH}_{4} \mathrm{Br}$, has been used to generate hydride-terminated Si NPs ${ }^{\text {[20d, 94] }}$ (Figure 
4.1). These Si-H NPs can be subsequently modified by hydrosilylation (HS) reactions, which involve the addition of unsaturated bonds across $\mathrm{Si}-\mathrm{H}$ surface to form more chemically robust $\mathrm{Si}-\mathrm{C}$ surfaces. The $\mathrm{Si} \mathrm{NPs}$ in this study were functionalized using the simple hydrocarbon, 1-decene to produce fluorescent hydrophobic Si NPs; fluorescent hydrophilic Si NPs were simultaneously produced (see below). It was hypothesized that the Si NPs in the hydrophobic layer possessed covalent decene-termination, while the hydrophilic Si NPs were electrostatically or covalently coated with the solvent, DMF, in addition to surface oxidation. This unintended surface modification of the Si NPs with the solvent complicates the ability to readily characterize the surface of the Si NPs and discern the effects of the NP size and surface on the optical properties.

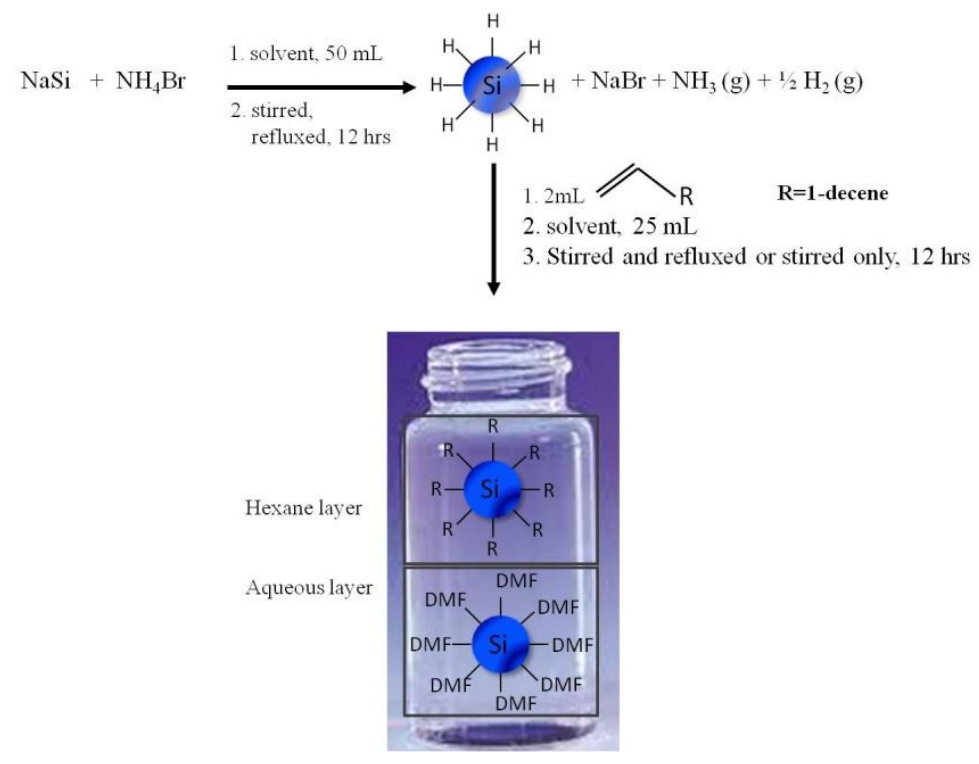

Figure 4.1. Scheme of the Zintl metathesis to prepare Si NPs. Zintl phase sodium silicide, NaSi, and ammonium bromide, $\mathrm{NH}_{4} \mathrm{Br}$, are reported to undergo a metathesis to produce $\mathrm{NaBr}, \mathrm{NH}_{3}(\mathrm{~g}), \mathrm{H}_{2}(\mathrm{~g})$, and hydride-terminated Si NPs, which may undergo HS with 1-decene to produce hydrophobic Si NPs. ${ }^{[20 d]}$ After HS, fluorescent hydrophilic Si NPs were produced alongside fluorescent, hydrophobic Si NPs. 
Chapter 4: Two-Step Metathesis and Hydrosilylation Reaction of Si NPs $\quad 60$

In efforts to determine if the interaction of the coordinating solvent with the NP surface could be avoided, the HS reaction with 1-decene was also performed, 1) using a chemical initiator, rather than heat, as well as 2) a thermal HS performed in a noncoordinating solvent (xylene). These reactions were aimed at minimizing the solventligand competition for the Si NP surface, which should enhance the capping efficiency by the 1-decene ligand.

\subsubsection{DMF-synthesized $\mathrm{Si}-\mathrm{H}$ NPs Prepared from the Metathesis of $\mathrm{NaSi}$ and $\mathrm{NH}_{4} \mathrm{Br}$}

The Si NPs obtained from the metathesis of $\mathrm{NaSi}$ and $\mathrm{NH}_{4} \mathrm{Br}$ in the presence of DMF were characterized prior to the HS reaction. The absorbance spectrum of this solution is shown in Figure 4.2 (line i). Typical of other Si NP solution, the spectra shows a broad absorption through the UV-Vis with an onset of absorbance at approximately $500 \mathrm{~nm}$, consistent with the indirect bandgap of $\mathrm{Si}^{[4,92 \mathrm{a}]}$ The broad shoulder at $260 \mathrm{~nm}(4.78 \mathrm{eV})$ has been associated with Si direct bandgap transitions. ${ }^{[4,20 \mathrm{~d}]}$

Under a handheld UV light (6 W, $365 \mathrm{~nm})$, the dried DMF-synthesized Si NP solution was observed to emit blue (Figure 4.2 inset). Using standard photoluminescent (PL) spectroscopy, the DMF-synthesized Si NPs have an emission maximum of $455 \mathrm{~nm}$ when excited with $370 \mathrm{~nm}$ light (Figure 4.2, line ii). The changes in the emission maxima as a function of excitation energy (lines iii-iv) have been previously observed and likely results from the excitation of different NP size populations with different absorption cross-sections with different excitation wavelengths. ${ }^{\text {[20d, 98] }}$ 


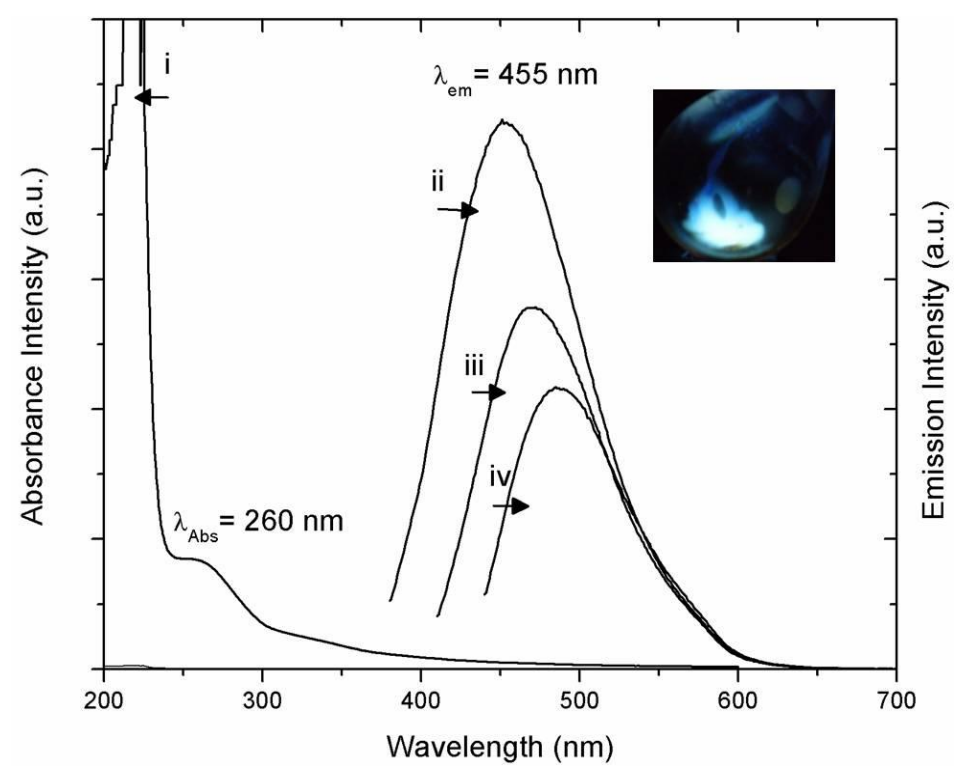

Figure 4.2. Photophysical properties of the DMF-synthesized Si NPs. Absorbance (i) and PL emission spectra of the DMF-synthesized Si NPs prepared from the metathesis of $\mathrm{NaSi}$ and $\mathrm{NH}_{4} \mathrm{Br}$ at increasing excitation wavelengths (ii-iv); $\lambda_{\mathrm{ex}}=370 \mathrm{~nm}$ (ii), $\lambda_{\mathrm{ex}}=400 \mathrm{~nm}$ (iii), $\lambda_{\mathrm{ex}}=430 \mathrm{~nm}$ (iv); Inset: Digital image of the DMF-synthesized Si NPs under a handheld UV lamp.

Representative TEM images of the DMF-synthesized Si NPs along with the histogram of the NP size distribution are shown in Figure 4.3. The TEM image indicates that the particles are all nearly spherical; most are well isolated, but a few appear to be aggregated. The inset in Figure 4.3a shows a fast Fourier transform (FFT) of the image of the DMF-synthesized Si NPs, and reveals that the NPs exhibit diffraction patterns due to their crystalline cores. ${ }^{[99]}$ This diffraction pattern shows reflections corresponding to the (111), (220), and (311) planes of diamond lattice Si at $3.14 \AA, 1.92 \AA$, and $1.64 \AA$, respectively (JCPDS card no. 001-0787). Measuring 350 NPs from various areas of the TEM grid, the average DMF-synthesized Si NP was determined to be $5.02 \pm 1.21 \mathrm{~nm}$; 
thus, much of the distribution of the Si NPs is smaller than the Bohr exciton radius of Si $(5.5 \mathrm{~nm}){ }^{[24]}$

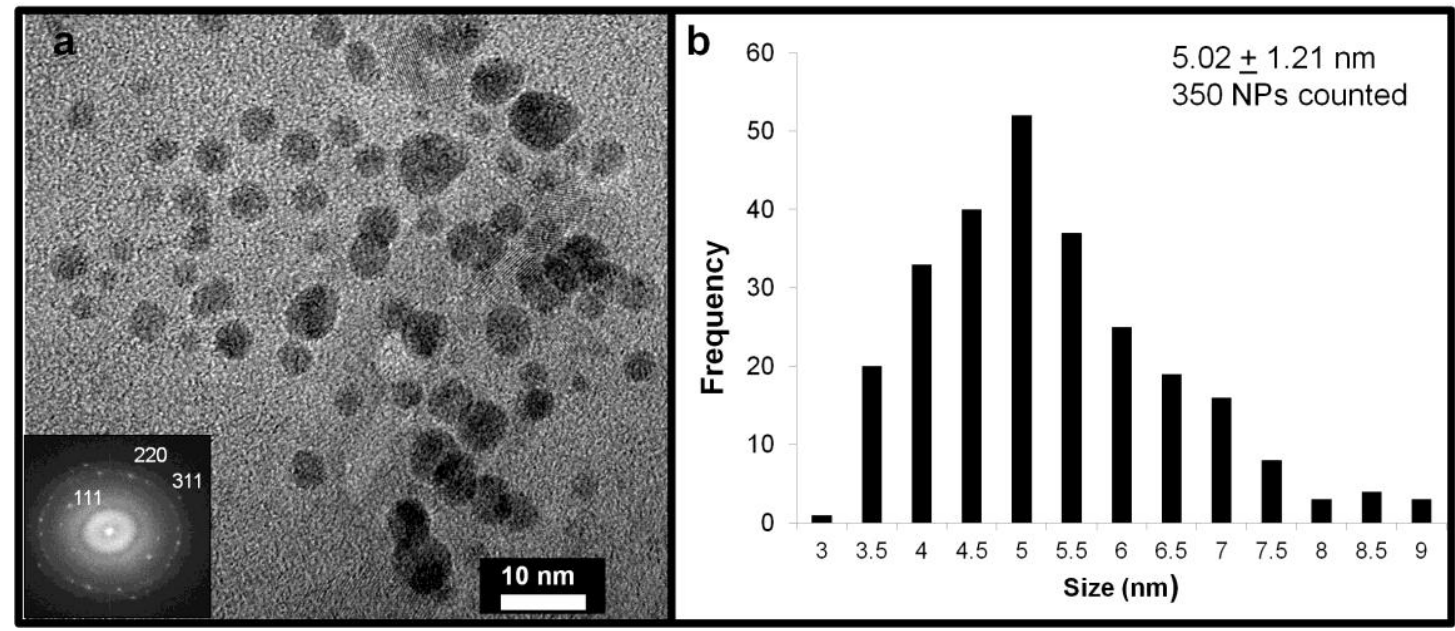

Figure 4.3. TEM image of DMF-synthesized Si NPs (a) with the corresponding histogram based on a survey of 350 NPs (b); Inset: FFT of the image of the DMF-synthesized Si NPs showing a diffraction pattern consistent with $\mathrm{Si}^{0}$.

Figure 4.4 shows the FT-IR spectra of neat DMF (line a, dashed) and the DMFsynthesized Si NPs (line b, solid). Neat DMF exists in two resonance forms (Figure 4.4, inset i); multiple FT-IR stretches are observed which correspond to both resonance forms, including a $\mathrm{C}=\mathrm{O}(\mathrm{s})$ at $1650 \mathrm{~cm}^{-1}, \mathrm{C}-\mathrm{N}(\mathrm{s})$ at $1250 \mathrm{~cm}^{-1}$, and a $\mathrm{C}-\mathrm{O}(\mathrm{s})$ at $1084 \mathrm{~cm}^{-1}$. The DMF-synthesized Si NPs were expected to be hydride terminated, as depicted in Figure 4.1, and is consistent with the small peak Si-H stretch at $2085 \mathrm{~cm}^{-1}$. ${ }^{[20 \mathrm{~d}]}$ There are also peaks at $1016 \mathrm{~cm}^{-1}$ and $806 \mathrm{~cm}^{-1}$; the higher energy peak may be consistent with a $\mathrm{C}$ O stretch, while the lower energy peaks can be assigned to the alkoxylation of the Si NP surface. The stretch in the $1100-1000 \mathrm{~cm}^{-1}$ region can also be consistent with the 
presence of Si surface oxidation, though the peak is much sharper than expected for this assignment.

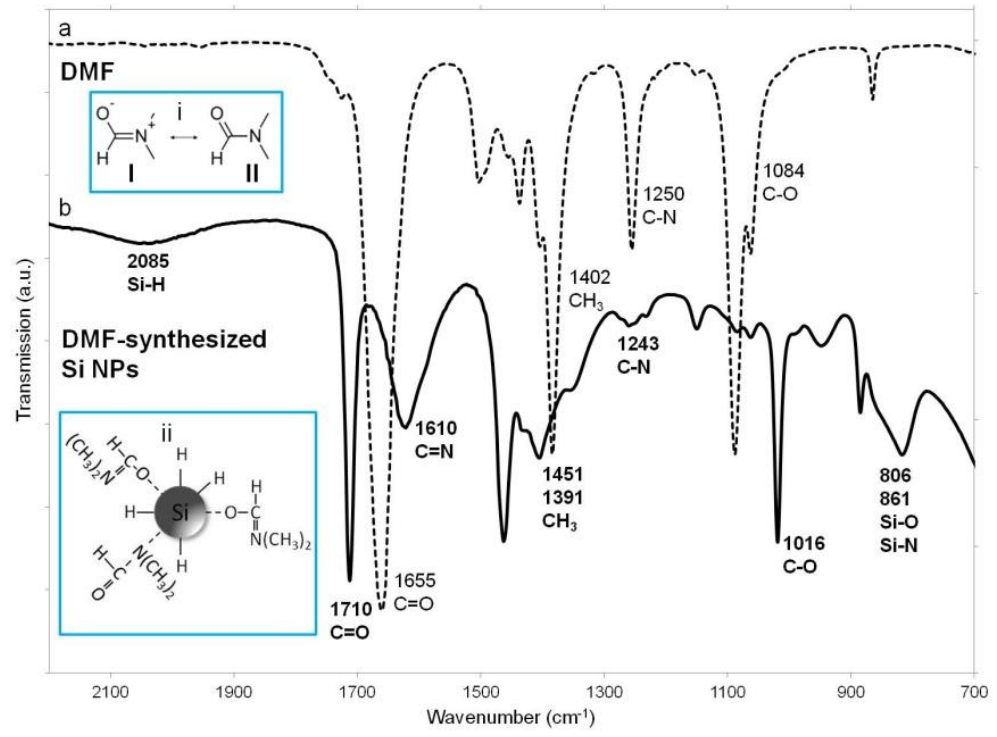

Figure 4.4. FT-IR spectra of neat DMF (line a, dashed) and DMF-synthesized Si-H NPs (line b, solid).

The frequencies and respective assignments are indicated, with the DMF-synthesized Si-H NP assignments in bold; Inset i shows the two resonance structures of DMF; Inset ii illustrates the possible configuration of the DMF-synthesized Si-H NPs with covalent hydride termination and coordinated DMF molecules.

It is possible that the Si NPs could become oxidized from adventitious oxygen; it is also possible that the solvent, DMF, is coordinated to the Si NP surface. According to the resonance structures, it seems possible that DMF could coordinate to the Si NP surface through the $\mathrm{O}$ or $\mathrm{N}$ atoms. Therefore, changes in the carboxylic acid or amine regions of the FT-IR of the DMF-synthesized Si NPs compared to neat DMF may be expected. The carbonyl region of the DMF-synthesized Si NPs has two obvious peaks, which may suggest that carbonyls in two distinct chemical environments exist. The higher energy peak at $1710 \mathrm{~cm}^{-1}$ may be assigned as a $\mathrm{C}=\mathrm{O}$, while the lower energy 
Chapter 4: Two-Step Metathesis and Hydrosilylation Reaction of Si NPs 64

stretch at $1610 \mathrm{~cm}^{-1}$ may be assigned as a $\mathrm{C}=\mathrm{N}$. These two peaks can coexist if DMF is coordinated to the Si NP surface through both resonance forms (i.e., when DMF in form I coordinates to the $\mathrm{Si}$ NP surface through the oxygen atom, a $\mathrm{C}=\mathrm{N}$ stretch is observed and when DMF in form II coordinates to the $\mathrm{Si} N \mathrm{NP}$ surface through the $\mathrm{N}$, a $\mathrm{C}=\mathrm{O}$ stretch is observed). This is supported by the possible alkoxylation and potential $\mathrm{Si}-\mathrm{N}$ stretch at $806 \mathrm{~cm}^{-1}$ and $861 \mathrm{~cm}^{-1}$, respectively. ${ }^{[20 \mathrm{~b}, 96,100]}$ The FT-IR spectrum of the DMFsynthesized suggests that these particles have a covalent $\mathrm{Si}-\mathrm{H}$ surface, alongside coordinated DMF in two different resonance forms (Figure 4.4, inset ii).

\subsubsection{Heat-Catalyzed HS Reaction of the DMF-synthesized Si-H NPs using 1-decene}

Though the FT-IR spectrum of the DMF-synthesized Si NPs exhibited evidence of surface passivation by DMF (as well as some surface oxidation, ) evidence for $\mathrm{Si}-\mathrm{H}$ termination is also observed, suggesting that some of the Si NP surface sites could still undergo HS with ligands of interest. A simple unsaturated hydrocarbon, 1-decene, was used to determine if the $\mathrm{Si}-\mathrm{H}$ surface sites could still undergo $\mathrm{HS}$, even in the presence of coordinated DMF. After the HS with 1-decene and subsequent hexane/water extraction workup, two separate layers exhibiting different emission characteristics were expected: a fluorescent hydrophobic layer containing the Si NPs that underwent successful HS with 1-decence, and a non-fluorescent (or less fluorescent) layer containing neat DMF along with Si NPs where the HS reaction was not successful. However, under a handheld UV lamp, the entire solution was fluorescent. A water/hexanes extraction was performed and both the hexane and water layers were fluorescent (Figure 4.5, Insets i and ii). The PL spectra revealed significantly blue shifted emission for both the water and hexane 
extraction layers (415 and $360 \mathrm{~nm}$, respectively) as compared to the DMF-synthesized SiH NPs (450 nm).

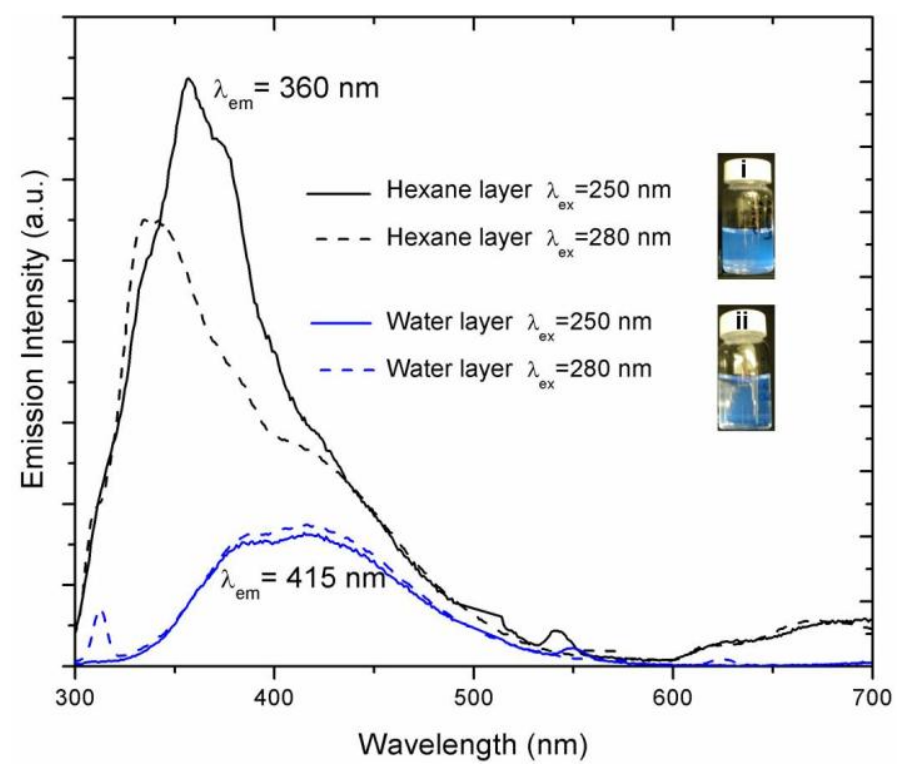

Figure 4.5. Emission spectra of the hexane (black) and water (blue) layers extracted from the HS of the DMF-synthesized Si NPs with 1-decene. Solid line, $\lambda_{\mathrm{ex}}=250 \mathrm{~nm}$; dotted line, $\lambda_{\mathrm{ex}}=250 \mathrm{~nm}$; Inset $\mathrm{i}$ and ii. Digital images of the hexane and water layers, respectively, from the HS of the DMF-synthesized Si-H NPs and 1-decene.

However, the maximum emission values were not all obtained using the same excitation wavelength. Interestingly, lower excitation wavelengths were required to generate appreciable emission intensity from both extracted layers $(<310 \mathrm{~nm})$, while the DMF-synthesized Si-H NPs required 340-400 nm excitation. If the emission of the Si NPs were strictly controlled by QC, then this would be expected to result from a decrease in the NP size upon HS. However, representative TEM images and histograms of the water and hexane layers of the Si NPs prepared from the HS of the DMF-synthesized SiH NPs and 1-decene (Figure 4.6) show no appreciable change in the size of the Si NPs 
after HS; the Si NPs extracted in the hexanes and water layers were determined to be $5.51 \pm 1.30 \mathrm{~nm}$ and 5.41 $\pm 1.21 \mathrm{NPs}$, respectively (the DMF-synthesized Si NPs were found to be $5.02 \pm 1.21 \mathrm{~nm})$. Because there is little change in the Si NP size, this lends support to factors other than particle size influencing the emission from Si NPs.

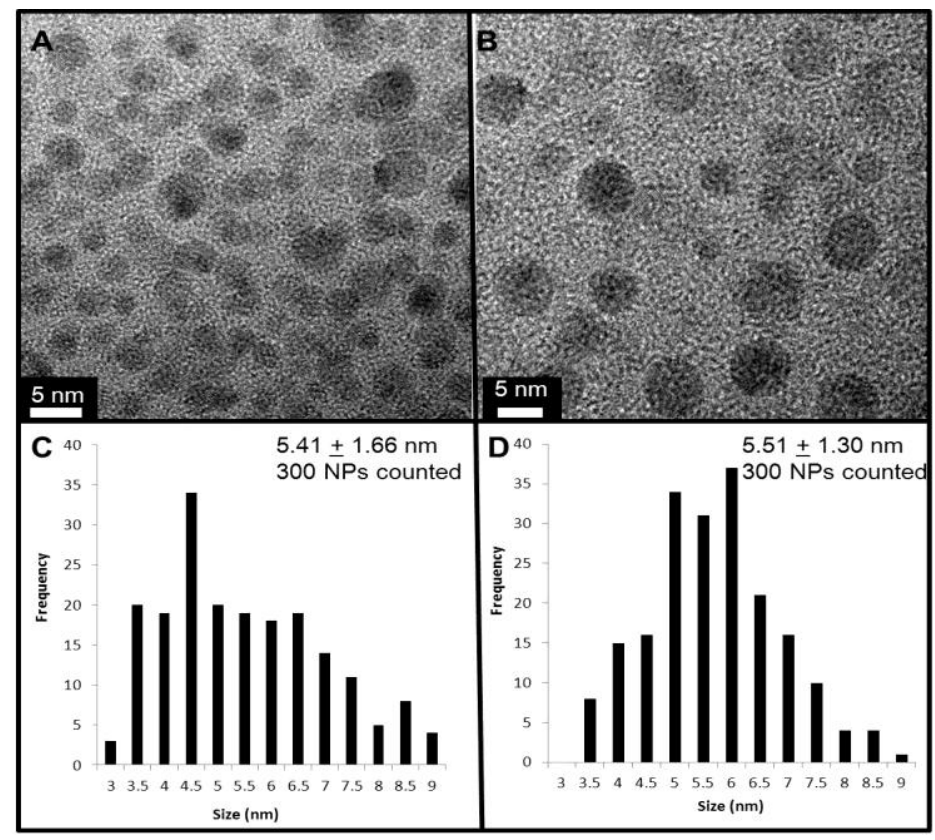

Figure 4.6. TEM images of the extracted hexane (A) and water (B) layers from the HS of the DMFsynthesized Si-H NPs with 1-decene with the corresponding histograms (C and D, respectively).

Because there was little change in the size of the Si NP after the HS reaction with 1-decene, it is unlikely that QC alone causes changes to the Si NP emission spectra. Variations in the Si NP surface chemistries have been shown to effect the emission. ${ }^{[18 b \text {, }}$ 97c] Since both the water and hexane extractions from the HS of the DMF-synthesized SiH NPs with 1-decene were fluorescent and possessed comparably sized Si NPs, it was believed that they must contain Si NPs with different surface chemistries, consistent with the difference in solubility. To investigate this, FT-IR was used to examine the surface 
chemistries of the hexane and water extractions of Si NPs. A successful HS reaction between the hydride-terminating surface groups of the DMF-synthesized Si NPs and 1decene would generate hydrophobic, decane-terminated Si NPs. The Si-H stretch, previously observed at $2085 \mathrm{~cm}^{-1}$, is now absent from the hydrophobic extraction of $\mathrm{Si}$ NPs (Figure 4.7A, line b). Combined with the new Si-C stretch ${ }^{25,35,40}$ at $1259 \mathrm{~cm}^{-1}$, the successful HS between the Si-H surface groups of the DMF-synthesized Si NPs and 1decene has likely occurred. It should also be noted that the $\mathrm{C}=\mathrm{C}$ (s) from 1-decene at $1641 \mathrm{~cm}^{-1}, 987 \mathrm{~cm}^{-1}$, and $907 \mathrm{~cm}^{-1}$ are also absent hydrophobic Si NP spectra. Si surface oxidation stretches and an alkoxylation bend at $1082 \mathrm{~cm}^{-1}$ and $1013 \mathrm{~cm}^{-1}$ and $793 \mathrm{~cm}^{-1}$, respectively, are still present, even dispersed in hexanes. A small $\mathrm{C}=\mathrm{O}(\mathrm{s})$ is also present at $1710 \mathrm{~cm}^{-1}$ in the hydrophobic Si NP extraction. Due to the immiscibility of DMF in hexanes, it is more likely that the decane-functionalized Si NPs are still coordinated with small amounts of DMF (inset Figure 4.7A).

The hydrophilic extraction from the HS of the DMF-synthesized Si-H NPs with 1decene was believed to contain Si NPs coordinated with DMF. As compared to the neat DMF-synthesized Si-H NPs, both spectra contained the $\mathrm{C}=\mathrm{O}$ and $\mathrm{C}=\mathrm{N}$ stretches at 1733 $\mathrm{cm}^{-1}$ and $1640 \mathrm{~cm}^{-1}$, respectively (Figure 4.7B). The Si-O stretch and alkoxyide bend at $1025 \mathrm{~cm}^{-1}$ and $780 \mathrm{~cm}^{-1}$, respectively, are much broader in the hydrophilic FT-IR spectrum, and is likely due to increased surface oxidation from the presence of water. However, even after the HS, potential Si-C or Si-N stretches around $1220-1200 \mathrm{~cm}^{-1}$ and $850 \mathrm{~cm}^{-1}$, respectively, are present. It is very unlikely that 1-decene could undergo HS to result in hydrophilic Si NPs, and therefore these peaks are likely indicative of a covalent 
bond between the Si NP surface and DMF. This suggests that the extracted hydrophilic layer from the HS of the DMF-synthesized Si-H NPs and 1-decene possess NPs with surface oxidation and DMF surface groups.

A

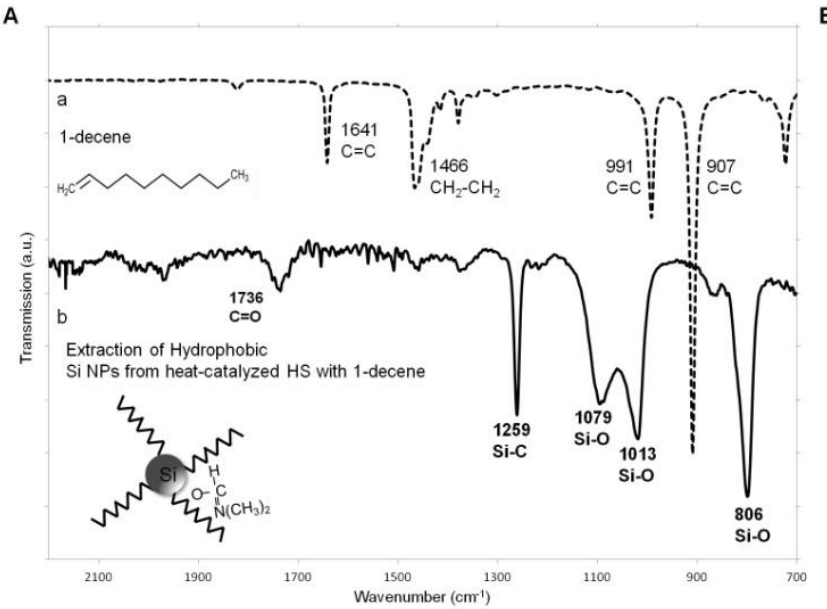

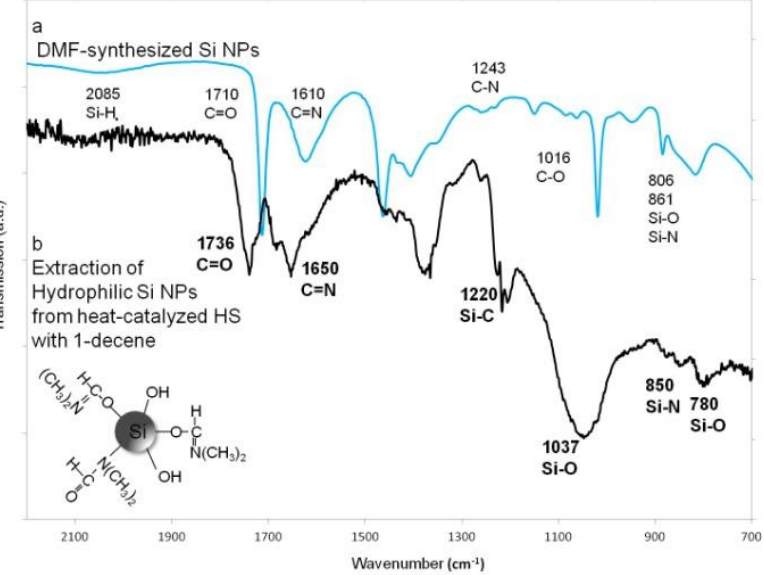

Figure 4.7. FT-IR spectra of the water and hexane extractions from the heat-catalyzed HS of the DMF-synthesized Si-H NPs with 1-decene. (A.) The hydrophobic layer obtained from the HS of the DMF-synthesized Si-H NPs with 1-decene was compared to neat 1-decene; Inset. The hydrophobic Si NPs may possess decane-termination alongside small amounts of DMF; (B.) The hydrophilic layer obtained from the HS of the DMF-synthesized Si-H NPs with 1-decene was compared to the neat DMF-synthesized Si NPs; Inset; The hydrophilic Si NPs may possess covalently bound DMF, as well as surface oxidation.

\subsubsection{Chemical-Catalyzed HS Reaction of the DMF-synthesized Si-H NPs using 1- decene}

In order to minimize the competition between the solvent and ligand for the surface of the Si NPs, the mode of HS initiation was varied. Not only have different catalysts been shown to possess different activities, ${ }^{[95 a, 101]}$ but due to the potential decomposition of DMF, heat was avoided. HS between the hydride-terminated $\mathrm{Si}$ 
surfaces and unsaturated hydrocarbons may also be initiated by chemical catalysts, ${ }^{[95 a]}$ and therefore, the DMF-synthesized Si-H NPs also underwent HS with 1-decene using the chemical catalyst, $\mathrm{K}_{2} \mathrm{PtCl}_{6}$. The effects on the emission properties in terms of surface chemistry by changing the initiator were examined.

Similar to the heat-initiated HS reaction between the DMF-synthesized Si-H NPs and 1-decene, the chemical-initiated HS reaction produced two fluorescent layers. Using the same high-energy excitation wavelengths as the heat-catalyzed reaction, the PL spectra was collected on the chemical-catalyzed HS extraction layers. The hydrophilic Si NPs from the heat- and chemical-catalyzed HS reactions were found to have almost identical emission spectra (Figure 4.8A), with maximum emission at $415 \mathrm{~nm}\left(\lambda_{\mathrm{ex}}=280\right.$ $\mathrm{nm})$. Because the emission spectra were so similar, it was believed that these species should have similar surface chemistries. However, the FT-IR spectrum of the hydrophilic Si NPs from the chemically catalyzed HS reaction with 1-decene did not resemble that of the heat-catalyzed HS (Figure 4.8B). The FT-IR spectrum of the hydrophilic Si NP layer extracted from the chemical initiated HS reaction possessed only one $\mathrm{C}=\mathrm{O}(\mathrm{s})$ at $1641 \mathrm{~cm}^{-}$ ${ }^{1}$, suggesting the presence of only one carbonyl environment. A small, potential Si-C(s) is observed at $1270 \mathrm{~cm}^{-1}$. While a C-O(s) is observed at $1084 \mathrm{~cm}^{-1}$, other peaks including Si-O and Si-N are difficult to assign. Small stretches in the $1041-1012 \mathrm{~cm}^{-1}$ and $890-860$ $\mathrm{cm}^{-1}$ regions are observed, but are not as definitive as the $\mathrm{Si}-\mathrm{O}(\mathrm{s})$ in the hydrophilic layer from the heat catalyzed HS reaction. 

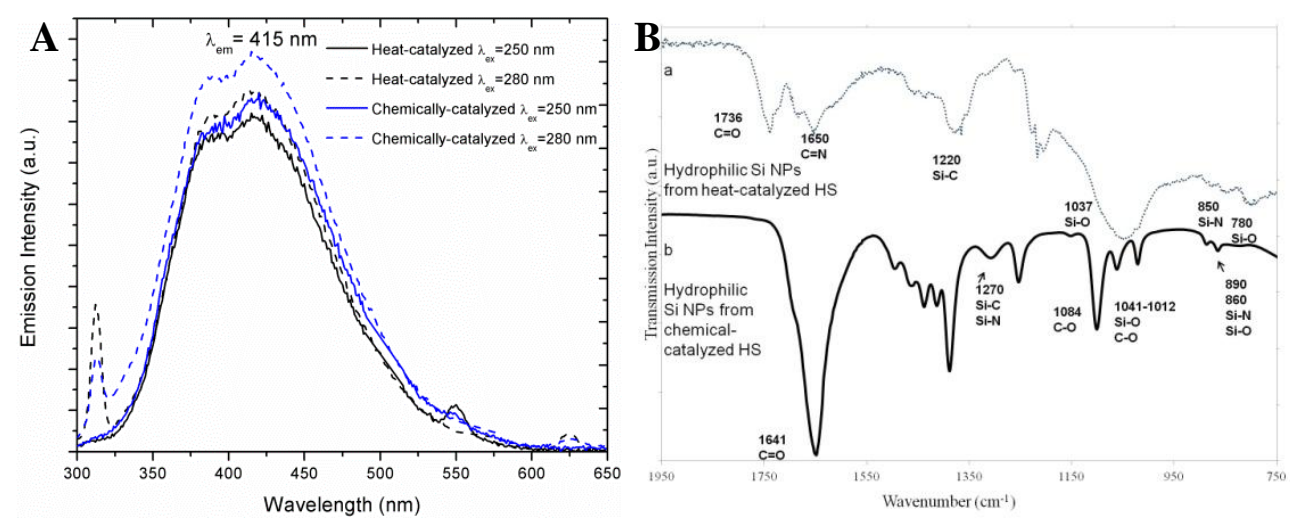

Figure 4.8. A. Emission $\lambda_{\mathrm{ex}}=250 \mathrm{~nm}$ (solid) and $\lambda_{\mathrm{ex}}=280 \mathrm{~nm}$ (dashed) and B. FT-IR spectra comparing the hydrophilic Si NPs from the heat-(black) and chemically-(blue) catalyzed HS reactions; B. FT-IR spectra of the hydrophilic Si NPs from the heat- (line a, dashed) and chemically- (line b, solid) initiated HS reaction.

The emission spectrum of the hydrophobic layer from the chemically-catalyzed HS of the DMF-synthesized Si-H NPs with 1-decene has a slightly blue-shifted emission maximum as compared to the heat-catalyzed hydrophobic Si NPs $(338 \mathrm{~nm}$ vs. $370 \mathrm{~nm}$, respectively, Figure 4.9A). However the emission intensity of the hydrophobic Si NPs prepared from the chemically initiated HS reaction is up to $50 \%$ lower at $\lambda_{\mathrm{ex}}=250$ or 280 nm. When the FT-IR spectrum of the hydrophobic layer from the chemically-catalyzed HS with 1-decene is examined, a small $\mathrm{Si}-\mathrm{C}(\mathrm{s})$ at $1273 \mathrm{~cm}^{-1}$ is observed, suggesting the successful HS of the Si NPs with 1-decene. Additionally, less intense Si-O and Si-O-C stretches are observed, as compared to the heat-catalyzed hydrophobic Si NPs, are observed. The remaining peaks $\left(1641 \mathrm{~cm}^{-1}, 991 \mathrm{~cm}^{-1}\right.$, and $\left.907 \mathrm{~cm}^{-1}\right)$ are assigned to neat 1-decene, which was not completely removed from the solution. Interestingly, this is no appreciable $\mathrm{C}=\mathrm{O}(\mathrm{s})$, suggesting that little $\mathrm{DMF}$ is coordinated to the Si NP surface. 

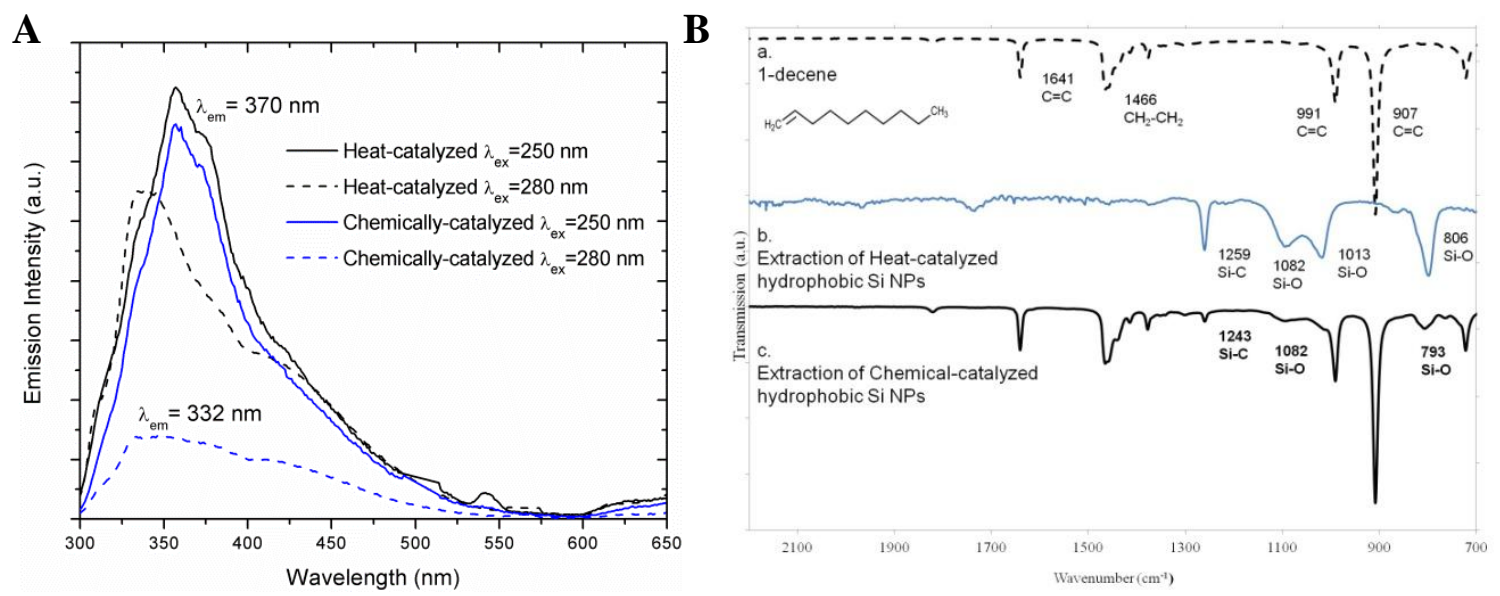

Figure 4.9. A. Emission using $\lambda_{\mathrm{ex}}=250 \mathrm{~nm}$ (solid) and $\lambda_{\mathrm{ex}}=280 \mathrm{~nm}$ (dashed) and B. FT-IR spectra comparing the hydrophobic Si NPs from the heat-(black) and chemically-(blue) catalyzed HS reactions; B. FTIR spectra of (a) neat 1-decene, (b) heat-catalyzed hydrophobic Si NPs and (c) chemically-catalyzed hydrophobic Si NPs.

Taken together, the spectroscopic evidence between the heat- and chemicallyinitiated HS reactions of the DMF-synthesized Si-H NPs with 1-decene suggests that the hydrophobic extractions possess successfully prepared decene-functionalized Si NPs. However, the intensity of the emission and the Si-C stretches in the FT-IR suggest that the decene-functionalized Si NPs prepared by the chemical-initiated HS reaction may be less efficient at preparing strongly emissive decene-functionalized Si NPs, as compared to the heat-initiated HS reactions. The hydrophilic Si NPs produced from the HS of the DMF-synthesized Si-H NPs and 1-decene possessed complicated FT-IR, regardless of the mode of HS initiation. Even with different surface chemistries, these solutions, interestingly, had very similar emission spectra, which only further complicate understanding the origin of the Si NP emission. 


\subsubsection{Heat-Catalyzed HS Reaction of Xylene-synthesized Si-H NPs and 1-decene}

While the chemically-initiated HS reaction between the DMF-synthesized $\mathrm{Si}-\mathrm{H}$ NPs and 1-decene did partially limit the competition between the ligand and the coordinating solvent, this reaction still produced hydrophilic Si NPs, which otherwise may have undergone HS with 1-decene potentially increasing the emission intensity from the decene-functionalized Si NPs. Though the chemical-initiated HS reaction produced the desired hydrophobic, decene-functionalized Si NPs, they did not exhibit significant emission intensities, as compared to the Si NPs from the heat-initiated HS reaction. This suggests that heat is required. Therefore, the synthesis of the Si-H NPs from the Zintl salt metathesis was also performed in the presence of a non-coordinating solvent with a comparable boiling point to DMF (boiling point $=155^{\circ} \mathrm{C}$ ); the reaction was performed in o-xylene (boiling point $=144{ }^{\circ} \mathrm{C}$ ) to determine if the desired hydrophobic, decenesynthesized Si NPs with strong emission intensity could be prepared.

The Zintl metathesis reaction of $\mathrm{NaSi}$ and $\mathrm{NH}_{4} \mathrm{Br}$ in xylene produced a fluorescent solution (that was understood to contain Si-H NPs), and was subsequently reacted with 1-decene via a heat-initiated HS reaction. The water/hexanes extraction produced two separate layers; the hydrophilic layer possessed minimal fluorescence (data not shown), which likely contained Si NPs with oxide and hydroxide termination.

The hydrophobic layer was fluorescent and had an emission maximum of $376 \mathrm{~nm}$ when excited using $250 \mathrm{~nm}$ (Figure 4.10). Unfortunately, the emission of the hydrophobic Si NPs from the synthesis in xylene only has an emission intensity that is $50 \%$ of the emission from the hydrophobic Si NPs prepared in DMF. This suggests that 
the heat initiated HS reaction in xylene is also not as efficient as the heat-initiated HS in DMF. When the FT-IR was examined (Figure 4.10), a sharp Si-C stretch at $1258 \mathrm{~cm}^{-1}$ was observed, along with significant surface oxidation and alkoxylation (1097 and 1008 $\mathrm{cm}^{-1}$ and $797 \mathrm{~cm}^{-1}$, respectively).
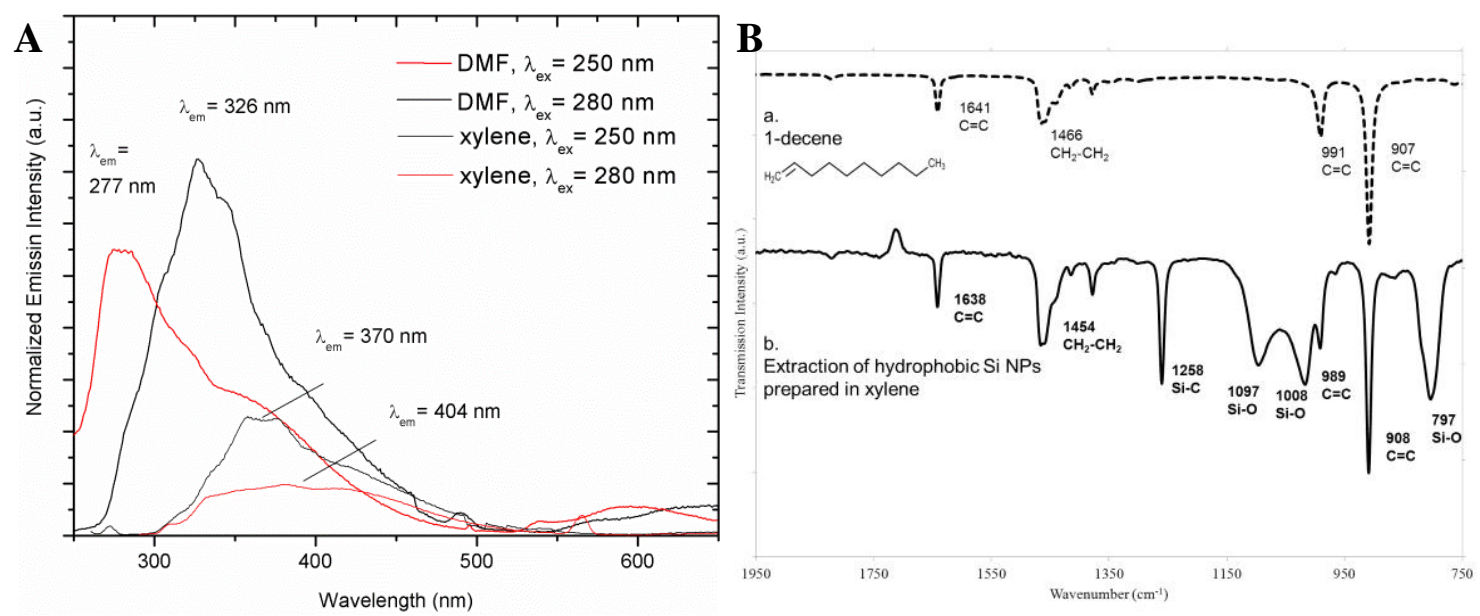

Figure 4.10. A. Emission and B. FTIR spectra of the hydrophobic Si NPs from the Zintl salt metathesis and HS reaction in xylene.

While the non-coordinating solvent allowed for the successful HS of the Si-H surface with 1-decene with minimal solvent competition, the decene-functionalized $\mathrm{Si}$ NPs were not very emissive. This low emission intensity may be due to significant surface oxidation, inefficient surface capping by 1-decene, or from the presence of only a few emissive Si NPs. It is possible that the coordinating solvent plays a vital role in the formation of the Si NPs from the Zintl salt metathesis, and is required to produce a significant number of Si NPs. 
Chapter 4: Two-Step Metathesis and Hydrosilylation Reaction of Si NPs 74

\subsection{Conclusions}

In addition to particle size, the emission of Si NPs is effected bythe surrounding media, including surface groups or solvents. Fairly monodisperse Si NPs $(5.02 \pm 1.21$ nm) with partial hydride-termination have been prepared using a solution based Zintl salt metathesis between $\mathrm{NaSi}$ and $\mathrm{NH}_{4} \mathrm{Br}$. In the presence of the coordinating solvent, $\mathrm{N}, \mathrm{N}$ 'dimethylformamide (DMF), thermal HS with 1-decene produces the anticipated hydrophobic Si NPs, while unintentionally producing hydrophilic Si NPs, showing evidence of coordination and termination by the solvent. Because all of the obtained $\mathrm{Si}$ NPs were, on average $5 \mathrm{~nm}$, the observed effects on the emission were not associated with quantum confinement, but rather with changes in the surface chemistry. In efforts to avoid the competition for the Si NP surface sites between the solvent and ligand, two other approaches for the HS with 1-decene were taken: 1) using a chemical initiator for the HS, rather than heat, and 2) synthesis of the Si-H NPs from the Zintl salt metathesis and subsequent HS reaction with 1-decene in the non-coordinating solvent, o-xylene. Using a chemical-initiated HS reaction in DMF resulted in hydrophobic Si NPs with decene-functionalization, but with low emission intensities. The chemical-initiated HS reaction also results in hydrophilic Si NPs with almost identical emission spectra as the heat-initiated HS; surprisingly, the surface chemistries were not identical, further complicating understanding the origin of the Si NP emission as it relates to the surface chemistry. The Zintl salt metathesis in xylene appeared to generate Si-H NPs, which underwent successful HS with 1-decene to produce hydrophobic Si NPs. Unfortunately, these particles were not very emissive, which was attributed to inefficient capping of the 
Si NPs or from the presence of very few Si NPs in solutions. From the reactions from the Zintl salt metathesis, the coordinating solvent appears to play a vital role in the formation of the Si NPs and is required to produce a significant number of Si NPs for subsequent functionalization. 


\title{
One-Step Zintl Salt Metathesis for Water Soluble, Photoluminescent, Surface- Oxidized Si Nanoparticles for Cellular Imaging Applications
}

\begin{abstract}
A versatile, one-step synthesis of water-soluble, highly emissive Si nanoparticles (NPs) using bi-functional, low-melting solids as reaction media has been developed. Characterization using transmission electron microscopy, selected area electron diffraction, X-ray photoelectron spectroscopy, and Raman spectroscopy show that the one-step melt synthesis produces nano-scale crystalline Si cores surrounded by a silicon oxide shell. Analysis of the NP surface using FT-IR, zeta potential, and gel electrophoresis indicate that the bi-functional low melting solid carboxylic acid is grafted onto the NP surface, which allows tuning of the particle surface charge, solubility, and functionality by also using a bi-functional amine as a solvent ligand. Photoluminescence spectra of the as-prepared Si NPs show an intense blue-green emission with a short (ns) lifetime suitable for biological imaging. These NPs are found to be stable in biological media and their cellular uptake and distribution was examined using live N2a cells.
\end{abstract}

This chapter is published as,

"One-step Melt Synthesis of Water Soluble, Photoluminescent, Surface-Oxidized Silicon Nanoparticles for Cellular Imaging Applications"

B.A. Manhat, A.L. Brown, L.A. Black, E.Rickman, J.B.A. Ross, K. Fitcher, T.Vu, A.M.Goforth. Chemistry of Materials. 2011, 23, 2407 


\subsection{Introduction}

Semiconductor nanoparticles (NPs) have received widespread interest for their efficient size-tunable wavelength light emission, which has led to the increased application of luminescent NPs in opto-electronic devices ${ }^{[5 c, 102]}$ and biological fluorescence imaging. ${ }^{[6 \mathrm{~d}, 103]}$ Considerable synthetic efforts have been placed on optimizing direct band-gap II-VI semiconductor quantum dots (QDs), especially those based on $\mathrm{Cd}^{2+}$, owing to their high luminescence quantum yields (QY) and tunable visible emissions, which are governed by a size-dependent quantum confinement (QC) effect. $^{[2,3 b, 10 c]}$ However, the electrochemical reactivity of binary QDs, as well as the toxicity of $\mathrm{Cd}^{2+}$, are concerning, ${ }^{[6 c, 104]}$ especially for intended uses in aqueous, biological imaging applications.

The discovery of photoluminescence (PL) from porous silicon ( $\mathrm{Si}$ ) in the early $1990 \mathrm{~s}^{[18 \mathrm{a}]}$ led to the study of nanostructured $\mathrm{Si}$ as efficient light emitters. Though the mechanism of the PL from Si is not yet fully controlled, freestanding Si or Si-based (e.g., oxidized $\mathrm{Si}$ ) nanoparticles (NPs) have the potential to overcome the aforementioned issues associated with using II-VI semiconductor QDs as biological fluorophores. Si has been established as a biocompatible element, ${ }^{[6 \mathrm{~b}, 105]}$ and because using covalent surface manipulations to impart various chemical functionalities on $\mathrm{Si}$ surfaces are welldocumented ${ }^{\left[93 \mathrm{~d},{ }^{95 b]}\right.}$ Si-based fluorophores are attractive for use in biological imaging applications. Additionally, several studies have already demonstrated that Si-based NPs exhibit high QYs, which are comparable to those of both organic fluorophores and Cdbased QDs. ${ }^{[106]}$ 
To date, several methods ${ }^{[19-20,20 d, 93 a-c, 106-107]}$ have been presented to achieve matrix compatible, surface-functionalized Si NPs, though most synthetic routes require at least two-steps. While laser synthesis in reactive vapor ${ }^{[108]}$ and mechano-chemical methods in liquid ligands ${ }^{[109]}$ have recently been found to produce emissive, surfacecapped Si NPs in a single step, these methods are not ideal for aqueous applications, since they have produced NPs with hydrophobic surface terminations. Though solution methods have produced NPs with more variable and biocompatible surface groups, unfortunately, solution methods, including the reduction of $\mathrm{SiX}_{4}(\mathrm{X}=$ halide) in microemulsions $^{[24,110]}$ and Zintl salt metathesis ${ }^{[20 \mathrm{~d}, 94]}$ or oxidation, ${ }^{[98 \mathrm{a},}{ }^{111]}$ have commonly required multiple steps to obtain water-soluble Si NPs. These reactions involve the generation of Si NP cores bearing reactive $\mathrm{Si}-\mathrm{H}$ or $\mathrm{Si}-\mathrm{X}$ surfaces followed by subsequent reaction steps, including hydrosilylation (HS) or Grignard reactions, to impart the Si NPs with the desired matrix compatibility properties.

To avoid the use of multiple reaction steps, a one-step synthesis of photoluminescent, Si NPs was developed using a novel melt procedure, which employs a neat, low melting dicarboxylic acid solid as a low-volume solvent and potential direct surface modifier in the Zintl salt, $\mathrm{NaSi}$, metathesis in the presence of ammonium bromide, $\mathrm{NH}_{4} \mathrm{Br}$. The versatility of this reaction is demonstrated by synthesizing Si NPs in numerous bi-functional solid melts or liquid solvents having a variety of chemical functional groups. Because the melt method limits the number of synthetic steps, as well as the number and quantity of chemicals used to synthesize Si NP colloids, this method is relatively environmentally friendly when compared to traditional wet chemical syntheses, 
which often require hazardous HF etching procedures or large volumes of organic solvents.

\subsection{Si NP Experimental and Characterization}

Materials. All synthetic manipulations were performed either in a $\mathrm{N}_{2}$-filled glovebox or under an Ar atmosphere using standard Schlenk techniques. All aqueous solutions were prepared using electrophoretically pure water $\left(\mathrm{nH}_{2} \mathrm{O}, 18 \mathrm{MOhm} \bullet \mathrm{cm}\right.$ resistivity). Prior to use, sodium silicide (NaSi, SIGNa, 80\%) was ball milled for 15 min under $\mathrm{N}_{2}$ in a tungsten carbide-lined milling vial with two- $1 \mathrm{~cm}$ tungsten carbide balls using a high energy mill mixer (Spex $8000 \mathrm{M})$. Ammonium bromide $\left(\mathrm{NH}_{4} \mathrm{Br}\right.$, Aldrich, $>$ 99.99\%) and glutaric acid $\left(\mathrm{C}_{5} \mathrm{H}_{8} \mathrm{O}_{4}\right.$, Aldrich, 99\%) were dried in vaccuo at $100{ }^{\circ} \mathrm{C}$ before use. Anhydrous citric acid $\left(\mathrm{C}_{6} \mathrm{H}_{8} \mathrm{O}_{7}\right.$, Fisher, 99.5\%) and anhydrous ethylenediamine $\left(\mathrm{C}_{2} \mathrm{H}_{8} \mathrm{~N}_{2}\right.$, Fisher, $\left.98 \%\right)$ were used as received in place of solid glutaric acid.

\subsubsection{Si NP Synthesis}

Synthesis and Purification of Si NPs in the neat glutaric acid (GA) melt: NaSi (9.8 mmol), $\mathrm{NH}_{4} \mathrm{Br}(19 \mathrm{mmol})$, and GA $(114 \mathrm{mmol})$ were loaded into a two-neck, roundbottomed flask inside an $\mathrm{N}_{2}$-filled glovebox. The closed reaction vessel was transferred to the Schlenk line, where the reaction mixture was heated to melt GA $\left(95-98{ }^{\circ} \mathrm{C}\right)$; the mixture was then brought to reflux $\left(200^{\circ} \mathrm{C}\right)$ for approximately $20 \mathrm{hrs}$, during which time a black suspension formed. After cooling to room temperature, the suspension solidified

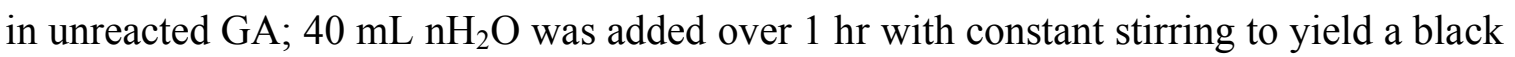
aqueous mixture. The black solid was removed by air-free vacuum filtration, leaving a 
yellow-colored solution containing the Si NPs. The black solid was identified as bulk, crystalline Si by powder X-ray diffraction.

Following isolation of the Si NPs in water, purification and analysis steps were performed in air. Excess GA and inorganic salts were removed from the Si NP solution by a combination of sublimation and dialysis. To remove GA, the Si NP solution was taken to dryness by rotary evaporation, and the resulting solid was subjected to sublimation using a cold finger (containing dry ice/acetone) vacuum apparatus on the Schlenk line. The sample was heated to the reduced boiling point of GA and left under dynamic vacuum for up to $3 \mathrm{hrs}$; a white solid was observed on the cold finger, which was subsequently identified as un-reacted GA. To reduce the amount of inorganic salts ( $\mathrm{NaBr}$ formed during reaction, or un-reacted $\mathrm{NH}_{4} \mathrm{Br}$ ) the aqueous $\mathrm{Si} \mathrm{NP}$ solution was dialyzed in $\mathrm{nH}_{2} \mathrm{O}$ up to three times using Fisher Brand hydrophilic dialysis tubing (regenerated cellulose, MWCO 1,000 Da).

To estimate the percent yield of Si NPs from this synthesis, the fluorescent, aqueous product was analyzed for Si content by ICP-MS. The percent yield of NP based on $\mathrm{Si}$ was $1 \%$, and assuming $4 \mathrm{~nm}$ particles (see below) with the density of bulk $\mathrm{Si}$, represensts approximately $10^{15}$ nanoparticles $/ \mathrm{mL}$. The ICP-MS analysis was performed by a contracted lab (Dr. Martine Ralle, Oregon Health \& Science University).

Synthesis of Si NPs in other neat, bi- or multi-functional ligands: Functionalized Si NPs were also synthesized in a citric acid (CA) melt by substituting the neat solid for GA in the above procedure. Additionally, the neat, bi-functional liquid ethylenediamine (en) was also used in the reaction in place of GA in the same relative molar quantities (1 
Chapter 5: One -Step Zintl Salt Metathesis of Water-Soluble, Emissive Si NPs 81

$\mathrm{NaSi}: 2 \mathrm{NH}_{4} \mathrm{Br}$ : 10 ligand). All synthetic procedures in alternate solvents are similar as those described above for the synthesis of Si NPs in GA. Excess CA and salts were removed by dialysis, while excess en was removed rotary evaporation, prior to dialysis to remove inorganic salts.

\subsubsection{Si NP Characterization}

Powder X-ray Diffraction (PXRD): PXRD measurements were used to identify solid products after synthesis and purification steps, as well as confirm the presence of $\mathrm{Si} 0$ domains in the aqueous Si NP samples. The samples were prepared by drop-casting the samples onto a Pt sample holder and examined on a Riguku Ultima IV X-ray diffraction system with graphite monochromatized $\mathrm{Cu} \mathrm{K}_{\alpha}$ radiation $(\lambda=1.54187 \AA)$ in the $20-70^{\circ} 2 \theta$ range.

Transmission Electron Microscopy (TEM), Energy Dispersive X-ray Spectroscopy $(E D X)$ and Selected Area Electron Diffraction (SAED): TEM and EDX measurements, performed on a Tecnai F-20 HR-TEM operating at $200 \mathrm{kV}$, were used to assess the morphology, size, size distribution, and composition of the as-prepared Si NPs. Topological EDX spectra for composition analysis were collected on isolated particles for 10 min with approximately $20 \%$ dead time between successive scans. Samples were prepared by drop casting aliquots of aqueous Si NPs onto 400-mesh holey carbon-coated copper grids (SPI), which were dried in air at $100^{\circ} \mathrm{C}$ overnight. Line-scanning EDX and SAED were performed on an FEI Titan 80-300 TEM operating at $300 \mathrm{kV}$. An EDX line scan was performed over a large, isolated Si NP (16 $\mu$ s dwell time with 20 points 
Chapter 5: One -Step Zintl Salt Metathesis of Water-Soluble, Emissive Si NPs 82

collected over the $20 \mathrm{~nm}$ length scale) to monitor the relative atomic ratios of $\mathrm{Si}$ and $\mathrm{O}$ as a function of position across the particle.

Raman Spectroscopy, X-ray Photoelectron Spectroscopy (XPS,) and Fourier Transform Infrared (FT-IR) Spectroscopy: Raman spectra were collected on a MicroRaman spectrometer (Jobin-Yvon LabRAM HR800 UV with 532 nm laser). Samples were prepared by drop casting Si standards and freshly purified Si NPs onto carbon tape adhered to glass slides $24 \mathrm{hrs}$ prior to data collection. XPS measurements were made on a ThermoFisher Escalab 250. Samples (which stood for several days) were prepared by drop casting the Si NP solution onto a clean Cr substrate, which was dried under ambient conditions. During the measurement, charge compensation was done with a flood gun and for analysis, the $\mathrm{C} 1 \mathrm{~s}$ peak was used as an internal reference set to $284.8 \mathrm{eV}$. FT-IR spectra were collected on a Nicolet iS10 spectrometer using a diamond ATR attachment. Aqueous solutions of Si NPs were drop cast onto the ATR crystal and the solvent was evaporated as necessary using a heat gun to deposit a film.

Zeta potential: Zeta potential measurements to examine the NP surface charge as a function of $\mathrm{pH}$ were collected on a Malvern ZS-90 using Zeta-sizer folded capillary cells with gold electrodes. A solution of GA- or CA-synthesized Si NPs was titrated with $0.1 \mathrm{M} \mathrm{NaOH}$ to obtain solutions with $\mathrm{pH}$ values ranging from 1.84-8.51. Conversely, ensynthesized $\mathrm{Si}$ NPs were titrated with $0.1 \mathrm{M} \mathrm{H}_{2} \mathrm{SO}_{4}$ to obtain solutions in the $\mathrm{pH}$ range of 10.06-7.06.

Gel Electrophoresis: A 1\% agarose gel was prepared using TAE buffer (40 mM tris buffer, $20 \mathrm{mM}$ acetic acid, $1 \mathrm{mM}$ EDTA, $\mathrm{pH}$ to 8.3 using $\mathrm{NaOH}$ ) with the well comb 
positioned in the center of the gel mold. The gel was allowed to polymerize for $1 \mathrm{hr}$ and samples of GA-, CA- or en-synthesized Si NP solutions (ranging in volume from $0.5 \mu \mathrm{L}-$ $3 \mu \mathrm{L}$ ) were mixed with $5 \mu \mathrm{L}$ of a $50 \%$ glycerol solution and loaded into the gel. The gel electrophoresis experiment was performed at a constant voltage $(50 \mathrm{~V})$ over $1 \mathrm{hr}$, and the gel was subsequently imaged in a UV light box (302 nm excitation) equipped with a digital camera.

UV-Vis Absorbance, Standard Photoluminescence (PL), and Time-resolved PL Spectrophotometry: UV-Vis absorption spectra were collected on a Shimadzu UV-2450 UV-Vis spectrophotometer while standard PL measurements were collected on a Shimadzu-RF5301PC spectrophotometer. QY values for the Si NPs were obtained relative to a standard organic fluorophore, 2-aminopyridine ( $\mathrm{QY}=60 \%)$, chosen on the based on the similarities in the absorbance and emission characteristics of the NPs (X) and 2-aminopyridine (ST). ${ }^{[12]}$ Solutions of 2-aminopyridine were prepared in $0.1 \mathrm{M}$ $\mathrm{H}_{2} \mathrm{SO}_{4}$ while the $\mathrm{Si} \mathrm{NPs}$ were diluted in $\mathrm{nH}_{2} \mathrm{O}$ from the as-prepared concentration. Serial dilutions were made until a series with linear absorbance values at $350 \mathrm{~nm}$ were obtained for both sample and standard, such that the maximum absorbance value did not exceed 0.1 . Emission spectra $\left(\lambda_{\mathrm{ex}}=350 \mathrm{~nm}, \lambda_{\mathrm{em}}=360-750 \mathrm{~nm}\right)$ were obtained for these dilutions, and the integrated PL emission intensities were plotted against the corresponding absorbance values. The slopes of these plots were used to determine the relative QYs, while the absolute Si NP QYs $\left(\Phi_{x}\right)$ were calculated by the following equation, $\Phi_{x}=\Phi_{S T}\left(\frac{\text { slope }_{x}}{\text { slope }_{S T}}\right)\left(\frac{\eta_{x}{ }^{2}}{\eta_{S T}{ }^{2}}\right)$, where $\Phi_{\mathrm{ST}}$ is the quantum efficiency of 2-aminophyridine, and $\eta$ is 
the refractive index. ${ }^{[112]}$ Time-resolved PL decay measurements were performed under magic-angle polarization conditions ${ }^{[113]}$ by time-correlated single-photon counting (TCSPC) using the FLASC 1000 (Quantum Northwest, Liberty Lake, WA) sample chamber. A frequency-doubled, ps-Mira 900 Ti:Sapphire laser (Coherent, Santa Clara, CA) provided pulsed excitation at $375 \mathrm{~nm}$ and at a repetition rate of $4.7 \mathrm{MHz}$. The emission was isolated through $25-\mathrm{nm}$ bandpass filters having peak transmission at 400 , 455, or $495 \mathrm{~nm}$ (Andover, Salem NH), respectively. The TCSPC data were collected at a timing resolution of 35 ps/channel using a Time Harp 200 PCI board (PicoQuant, Berlin), and each intensity decay curve was collected to $4 \times 10^{4}$ at the peak. An instrument response function was also collected to equivalent peak counts, using a light-scattering solution of dilute colloidal silica.

It was assumed that the intensity decay, $I(t)$, could be adequately described by sums of exponentials. In this case, $I(t)=\sum_{i=1}^{n} \alpha_{i} e^{-t / \tau_{i}}$, where $\tau_{i}$ is the lifetime and $\alpha_{i}$ is the amplitude of the $i$ th component in the intensity decay. The intensity-weighted lifetime is then $\left\langle\tau>=\sum_{i=1}^{n} \alpha_{i} \tau_{i}^{2} / \sum_{i=1}^{n} \alpha_{i} \tau_{i}\right.$. The data were fit by non-linear least-squares using iterative reconvolution (software package FluoFit Pro V4.2.1 (PicoQuant, Berlin)), and the statistical goodness of fits was assessed by the reduced chi squared value, randomness of the residuals, and autocorrelation of the residuals. ${ }^{[113-114]}$

Cellular Imaging: The GA-synthesized $\mathrm{Si}$ NPs were imaged using a neuroblastoma cell line derived from mice, Neuro-2a (N2a) (ATCC). Cells were cultured in a medium comprised of 47.5\% Dulbecco's Modified Eagle's Medium, 47.5\% Opti- 
MEM, and 5\% fetal bovine serum. Two days prior to imaging, cells were plated at a $40,000 \mathrm{cell} /$ well density in a 6 -well plate and protected by a $25-\mathrm{mm}$ poly-D-lysine clover slip. To examine the cellular uptake, the medium was removed and replaced with a concentrated sample of GA-synthesized Si NPs $(1 \mathrm{~mL} /$ well $)$ in PBS $(\mathrm{pH}=7.4)$. The cells were then incubated in the NP solution for $1 \mathrm{hr}$ at $37^{\circ} \mathrm{C}$ before washing the cells $2-3$ times with PBS. The cells were imaged on a Zeiss Axiovert 200M inverted microscope fitted with a 100x objective $(\mathrm{NA}=1.4)$ using a QD525 filter set from Chroma (Rockingham, VT) with a $400 \pm 60 \mathrm{~nm}$ excitation filter, a $475 \mathrm{~nm}$ long pass dichroic filter, and a $525 \pm 40 \mathrm{~nm}$ emission filter. Z-stack images of live N2a cells were acquired in both differential interference contrast (DIC) and emission modes using a monochrome CCD Axiocam camera. The spacing between each slice was $275 \mathrm{~nm}$. Control cells (without the addition of the GA-synthesized Si NPs) were also imaged in the same way.

\subsection{Results and Discussion}

Conventional Zintl metatheses between metal silicides and ammonium halides have been previously performed within sealed tubes at high temperatures $\left(\mathrm{T}>300^{\circ} \mathrm{C}\right)$, or alongside the presence of coordinating solvents at lower temperatures $\left(\mathrm{T}<300{ }^{\circ} \mathrm{C}\right)$. ${ }^{[20 \mathrm{~d} \text {, }}$ 115] While Zintl salt metathesis reactions have produced hydride-terminated Si NPs that can undergo subsequent hydrosilylation reactions, it was found that the coordinating solvent, (N,N-dimethylformamide, DMF) would compete with an unsaturated ligand for the Si NP surface. This competition not only generated undesirable solubility of the Si NPs, but also provided surface groups that were not amenable to biological applications. 
However, if a di-carboxylic acid were used as a solvent in the Zintl salt metathesis, it is anticipated that one carboxylic acid group would coordinate to the Si NP surface, while the other $\mathrm{COOH}$ group can impart the Si NP with aqueous solubility and remain available for the possible interaction with biological molecules. The one-step synthesis method described here utilizes low melting (or boiling), bi- functional organic molecules to serve as both the reaction medium (upon melting) and prospective surface terminating groups (Figure 5.1).

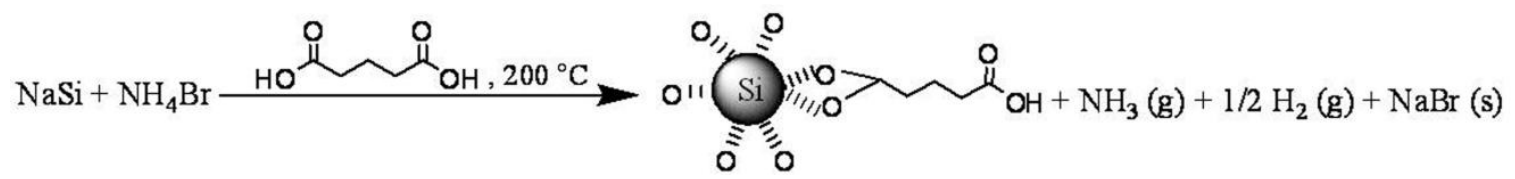

Figure 5.1. Representative one-step melt synthesis of Si NPs in GA (reagents, solids; products, aqueous after addition of water to solid product). Citric acid (CA) and ethylenediamine (en) can be substituted for GA.

\subsubsection{Characterization of Glutaric Acid (GA)-synthesized Si NPS}

Representative TEM images of the one-step, melt-synthesized Si NPs prepared in GA are shown in Figure 5.2 ( $a$ and $b$ ); the particles produced by this method appear spherical, abundant, and are relatively monodisperse. Measuring 1020 NPs from various areas of the TEM grid, the particles were determined to be $3.9 \pm 0.8 \mathrm{~nm}$ (see histogram in Appendix); thus the majority of the NPs are smaller than the Bohr exciton radius of $\mathrm{Si}^{[24]}$ HR-TEM (inset, Figure 5.2b) indicates that the Si NP cores are crystalline, however indexing the lattice fringes exhibited by these particles were consistent with more than only the presence of diamond lattice Si. This has been observed previously in 
other reports of oxide-embedded crystalline Si NPs, and has been attributed to forced invasive oxidation and lattice strain. ${ }^{[116]}$ The SAED pattern (Figure 5.2c) shows multiple rings consistent with diamond lattice $\mathrm{Si}^{[99]}((111)$ at $3.14 \AA,(220)$ at $1.92 \AA,(311)$ at 1.64 $\AA$, and (400) at $1.34 \AA$; JCPDS card 001-0787). In addition, diffraction rings consistent with crystalline $\mathrm{SiO}_{2}$ are observed at $3.4 \AA, 2.1 \AA$ and $1.8 \AA$ ((011), (111), and (112), respectively, marked with *, JCPDS card no. 087-2096). The presence of $\mathrm{SiO}_{2}$ is attributed to the partial oxidation of the Si NPs, which are potentially capped with an oxygen-containing organic material, surface oxide, surface hydroxide, or a combination of these.

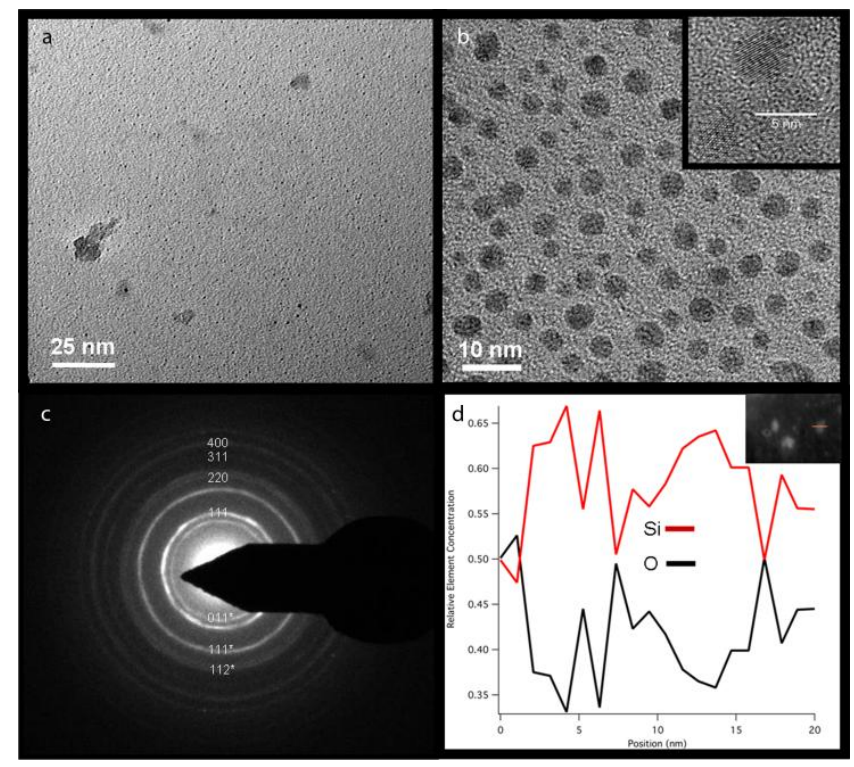

Figure 5.2. TEM images of the GA-Si NPs. (a,b) TEM images of GA-synthesized Si NPs showing welldispersed, spherical particles; (Inset b) HR-TEM image showing a high degree of crystallinity in the NP cores, scale bar $5 \mathrm{~nm}$; (c) SAED pattern of NPs showing the corresponding diamond $\mathrm{Si}$ and $\mathrm{SiO}_{2}(*)$ diffraction rings; (d) EDX line scan monitoring the relative $\mathrm{O}$ and $\mathrm{Si}$ concentrations across a particle, suggesting oxidized surfaces and $\mathrm{Si}^{0}$ rich cores, (Inset d) dark field TEM image of isolated NP on which EDX line scan was performed, scale bar $20 \mathrm{~nm}$. 
EDX on the purified GA-synthesized NP products reveal the characteristic X-rays of $\mathrm{Si}, \mathrm{O}, \mathrm{C}, \mathrm{Cu}$ (grid), and small amounts of $\mathrm{Na}$ and $\mathrm{Br}$ (starting material and salt byproduct, see Appendix). The high counts from $\mathrm{C}$ and $\mathrm{O}$ are expected: from the $\mathrm{Cu}$ grid coating $(\mathrm{C})$, from adventitious atmosphere $(\mathrm{O})$, from un-reacted GA that may remain solution after purification ( $\mathrm{C}$ and $\mathrm{O})$, or from GA bound to Si NPs (C and O). Therefore, the presence of $\mathrm{C}$ and $\mathrm{O}$ observed by EDX does not indicate any particular mode of GANP association. A subsequent EDX line scan was performed over a large, isolated, 20 $\mathrm{nm}$ Si NP (inset, Figure. 5.2d) to monitor the relative atomic ratios of $\mathrm{Si}$ and $\mathrm{O}$ as a function of position across the particle. These measurements indicate an $\mathrm{O} / \mathrm{Si}$ ratio far less than 2:1 (expected for pure $\mathrm{SiO}_{2}$ ), and in general, far less than 1:1, which suggests $\mathrm{Si}^{0}$ rich regions. Furthermore, as the beam was swept across the particle, the measured $\mathrm{O} / \mathrm{Si}$ ratios generally did not approach 1 (consistent with $\mathrm{SiO}_{2}$ ) until the edges of the NP were reached, suggesting that the surfaces are more heavily oxidized than the cores.

Raman spectroscopy was performed on a macroscopic sample of freshly purified GA-synthesized Si NPs (Figure 5.3). The maximum of the vibration band was found at $515.2 \mathrm{~cm}^{-1}$ for the $\mathrm{Si} \mathrm{NP}$ sample, which is close to the Si-Si vibration from bulk, crystalline Si at $516 \mathrm{~cm}^{-1} .^{[108]}$ This slight blue shift, which has been previously observed for other Si NPs, ${ }^{[108]}$ is attributed to the smaller domain size of nanosized Si. The Si NPs do not exhibit a peak in the $\mathrm{SiO}_{2}$ region $\left(498 \mathrm{~cm}^{-1}\right)$, which suggests the absence of amorphous $\mathrm{SiO}_{2}$ the freshly prepared GA-synthesized NP sample. 


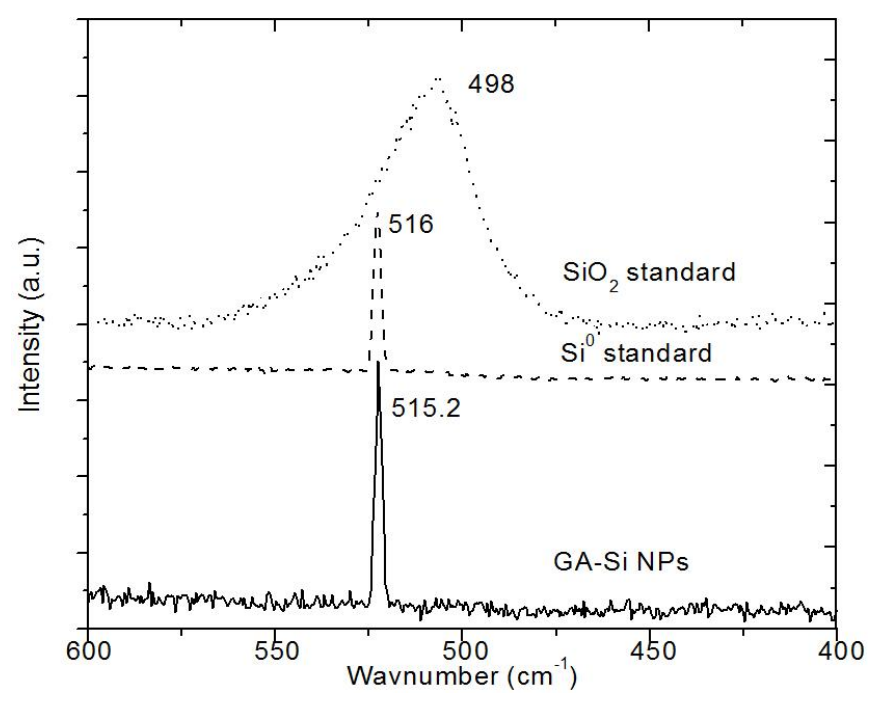

Figure 5.3. Raman spectra of freshly prepared GA-synthesized Si NPs (solid) compared to Si (dashed) and $\mathrm{SiO}_{2}$ (dotted) standards, confirming that the NP sample contains $\mathrm{Si}^{0}$ domains.

To verify the presence of $\mathrm{Si}^{0}$ domains observed by Raman spectroscopy, PXRD analysis was performed on a purified aqueous Si NP sample obtained from the GA melt. The XRD pattern (Figure 5.4) shows the expected reflections corresponding to the (111) and (220) planes of diamond lattice Si (28.12 and 47.12 degrees $2 \theta$, respectively), in good agreement with the corresponding calculated pattern (at 28.47 and 47.34 degrees $2 \theta$, respectively). These peaks are weak in intensity relative to those of the Pt sample holder; thus higher angle reflections are not visible above noise. Due to the low intensity of the observed Si NP peaks, the crystallite size based on line broadening was not performed. 


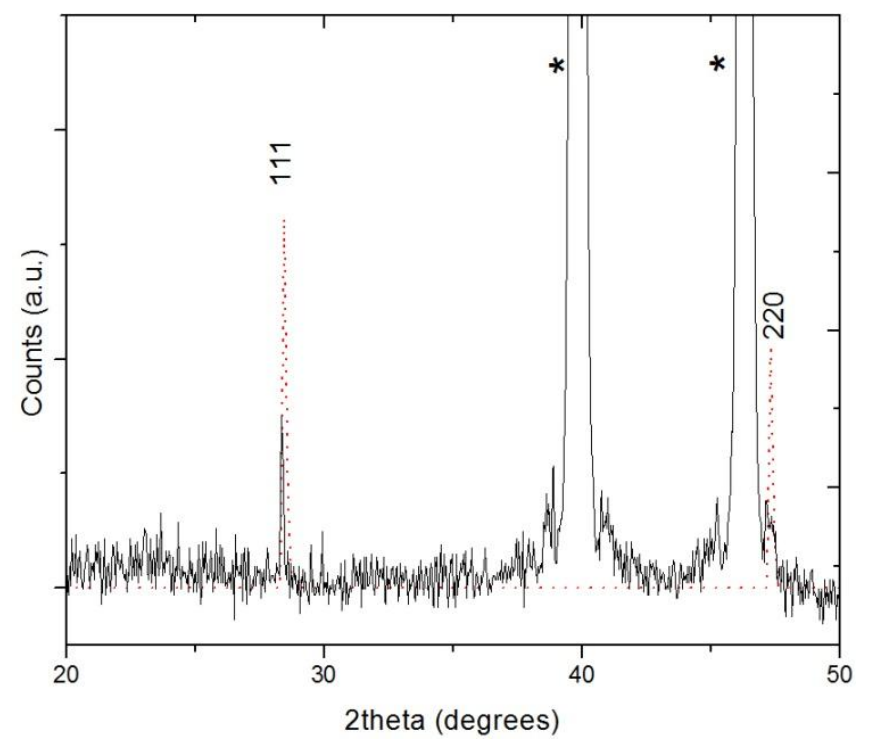

Figure 5.4. XRD pattern of GA-synthesized Si NPs (solid) compared to a calculated Si powder

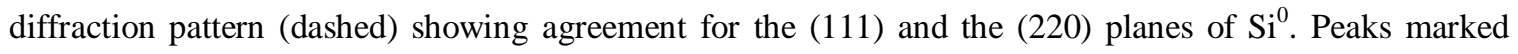
with * belong to the Pt sample holder.

XPS survey scan detected the presence of $\mathrm{Si}, \mathrm{C}, \mathrm{O}, \mathrm{Br}$, and $\mathrm{Cr}$ foil (Figure 5.5A). The $\mathrm{Si} 2 \mathrm{p}$ region (Figure 5.5b) show a single, broad $\mathrm{Si}$ peak that was assigned as $\mathrm{SiO}_{\mathrm{x}}(\mathrm{x}$ < 2) based on the absence of loss features expected for crystalline Si $(99 \mathrm{eV})^{[117]}$; the calibrated, energetic position of the Si $2 p$ peak was found to be ca. $102 \mathrm{eV}$. It is unlikely that charging was an issue in accurately determining the $\mathrm{Si} 2 \mathrm{p}$ binding energy, and therefore, the GA-synthesized $\mathrm{Si}$ NPs are assigned a $\mathrm{SiO}_{\mathrm{x}}$ surface. This assignment is further supported by the separation between the C 1 s and $\mathrm{Si} 2 \mathrm{p}$ signals; this separation should be approximately $186 \mathrm{eV}$ assuming that the $\mathrm{C} 1$ s signal at $284.8 \mathrm{eV}$ is dominated by adventitious carbon. ${ }^{[18]}$ Here, the separation between the C $1 \mathrm{~s}$ and the Si $2 \mathrm{p}$ signal was found to be slightly less than $183 \mathrm{eV}$, suggesting that the calibrated position of the $\mathrm{Si}$ 2p peak was $\sim 102 \mathrm{eV}$, again, indicative of $\mathrm{SiO}_{\mathrm{x}}$. The results of the XPS are consistent 
with a high level of oxidation on the Si NP surface as are the results of SAED and EDX analyses. Additionally, the C 1s region of the XPS spectrum (Figure 5.5c) also shows a peak at $288 \mathrm{eV}$, consistent with carboxylate carbon. ${ }^{[119]}$ Therefore, the collective results support the hypothesis that small, crystalline $\mathrm{Si}^{0}$ regions are present in the core of the particles and are surrounded by an oxidized silicon surface on which the solvent may have been adsorbed. With the Si NPs larger than the expected mean free path of the ejected electrons, ${ }^{[107 \mathrm{e}]}$ absence of crystalline Si loss features is not unexpected given that the $\mathrm{Si}^{0}$ regions might not be detected because they are deeply embedded.
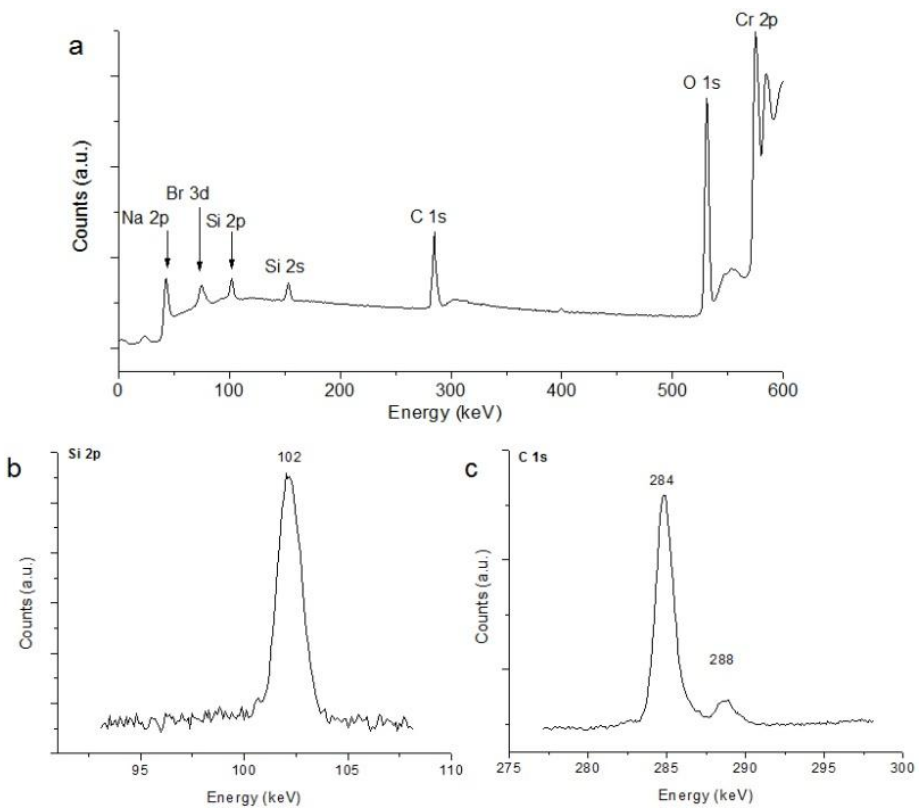

Figure 5.5. XPS scans of the GA-synthesized Si NPs. (a) survey scan detecting the presence of Cr (foil), $\mathrm{O}, \mathrm{N}, \mathrm{C}, \mathrm{Si}, \mathrm{Br}$, and Na; (b) Si $2 \mathrm{p}$ region has one peak centered at $\sim 102 \mathrm{eV}$, indicating an $\mathrm{SiO}_{\mathrm{x}}$ surface assignment; (c) $\mathrm{C} 1 \mathrm{~s}$ region shows two peaks at $284 \mathrm{eV}$ and $288 \mathrm{eV}$ from adventitious carbon and carboxylate species, respectively. 
Chapter 5: One -Step Zintl Salt Metathesis of Water-Soluble, Emissive Si NPs 92

FT-IR was used to examine the role of the solvent ligand more closely, as well as examine the effects of prolonged air exposure. The FT-IR spectra $\left(600-1800 \mathrm{~cm}^{-1}\right)$ of neat GA, freshly purified GA-synthesized Si NPs, and aged purified GA-synthesized Si NPs are shown in Figure 5.6 (full spectra, 400-4000 $\mathrm{cm}^{-1}$, Appendix). To provide surface coverage and minimize surface energy upon NP formation, it was anticipated that GA would covalently bond to the $\mathrm{Si}$ or $\mathrm{SiO}_{x} \mathrm{NP}$ surface. For each potentially surfacebound GA molecule, steric arguments would suggest that one $-\mathrm{COOH}$ group would interact with the NP surface, leaving the other group at the NP/solvent interface available to interact with other NPs or available for subsequent functional group manipulations (e.g., peptide addition by carbodiimide coupling). Therefore, a change in the carbonyl region (relative to neat GA) would be expected for the GA-synthesized Si NPs if GA were covalently bound to the $\mathrm{Si}$ or $\mathrm{SiO}_{\mathrm{x}}$ surface through one or more of its acid groups. The carbonyl region of the Si NP spectrum (shaded in green) is broadened and split (from $1680 \mathrm{~cm}^{-1}$ to $1700 \mathrm{~cm}^{-1}$ and $1575 \mathrm{~cm}^{-1}$, shaded in purple) relative to that of neat GA, which may indicate a change in the ligand $\mathrm{C}=\mathrm{O}$ environment. Similar spectroscopic features have been previously observed in the carbonyl regions of other samples with carboxylated surfaces, including: carboxy-terminated Ge (100) $2 \times 1$ surfaces, ${ }^{[120]}$ carboxylic acid-grafted silica NPs, ${ }^{[121]}$ and other carboxy-terminated Si NPs. ${ }^{[20 a]}$ In the latter report, a shift of the carbonyl signal to $1600 \mathrm{~cm}^{-1}$ occurred upon covalent carboxylic acid attachment, similar to the shift to $1575 \mathrm{~cm}^{-1}$ observed in the case of the GA-synthesized Si NPs; thus, the FT-IR results are supportive of GA interacting with the NP surface in a bi-dentate manner. In addition, Kim et al. report the appearance a Si-O-C 
vibration $^{[121]}$ at $1083 \mathrm{~cm}^{-1}$, which should also be present in our product spectra if GA is covalently bound to the NPs. In the Si NP product spectrum, there is a strong, broad peak in that region, centered at $1077 \mathrm{~cm}^{-1}$ as well as a $\mathrm{Si}-\mathrm{O}$ bend ${ }^{[122]}$ at $800 \mathrm{~cm}^{-1}$ (shaded in pink). These peaks are consistent with partially oxidized Si NPs; however, the two possibilities ( $\mathrm{Si}-\mathrm{O}-\mathrm{C}$ or $\mathrm{Si}-\mathrm{O}$ ) cannot be differentiated due to the broad nature of the $\mathrm{Si}-\mathrm{O}$ stretch. These features both increase in magnitude upon aging the sample in air, suggestive of increasing oxidization of the surface with time. Regardless of the initial surface passivation, which may be a mixture of covalent GA, oxide, or hydroxide, this growth suggests that Si NPs eventually exhibit a heavily oxidized surface. Curiously, the -OH vibrations (shaded in blue) at 1372 and $917 \mathrm{~cm}^{-1}$ are found to decrease with increased levels of oxidation. While the split carboxylate region suggests at least partial surface coverage by GA, the breath of the Si-O peak at $1077 \mathrm{~cm}^{-1}$ precludes definitive assignment of a surface grafted Si-O-C feature. 


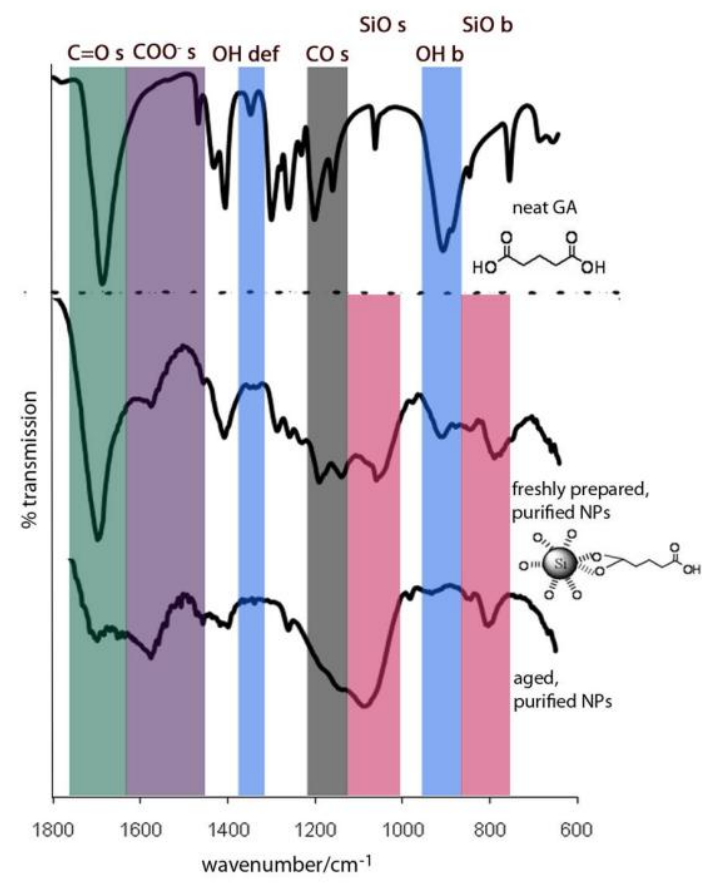

Figure 5.6. Fingerprint regions of the FT-IR spectra for neat GA (top), freshly prepared, purified Si NPs (middle), and aged, purified Si NPs (bottom). The sample spectra show characteristics of surface bound bi-dentate carboxylate in addition to surface oxidation, which increases over time.

Zeta potential measurements were made on a series of GA-synthesized Si NPs at varying $\mathrm{pH}$ values to compare the surface charge characteristics, which can describe the surface bound species. As shown in Figure 5.7, the GA-synthesized Si NPs exhibit a positive zeta potential at low $\mathrm{pH}$ values, consistent with the protonation of the carboxylic acid group. As the solution is titrated to a higher $\mathrm{pH}$, a gradually increasingly negative potential is observed due to the increasing partial deprotonation of the carboxylate group, consistent with Le Chatelier's Principle. While the GA-Si NP zeta potential becomes slightly less negative at $\mathrm{pH}$ values greater than the $\mathrm{pKa}\left(\mathrm{pK}_{\mathrm{a} 2} \mathrm{GA}=5.41\right)$, this supports the presence of GA molecules on the Si NPs. To corroborate this, gel electrophoresis was 
performed on the GA-synthesized Si NPs. The inset of Figure 5.7 shows the migration of GA-Si NPs when voltage is applied. Because the GA-synthesized Si NPs are emissive (see below), staining or processing steps were not necessary to image the gel. The movement of the fluorescent Si NPs is consistent with the zeta potential results: the GAsynthesized NPs (at $\mathrm{pH}$ 8.3) show a trend of migration toward the positive electrode, suggesting that they are negatively charged. Thus, based on all the analytical evidence, it seems most likely the GA-synthesized $\mathrm{Si}$ NPs consist of $\mathrm{Si}^{0}$ nanocrystals residing in an oxide matrix and covered by surface-bound solvent molecules that do not completely passivate the surface, allowing it to become increasingly oxidized with time.

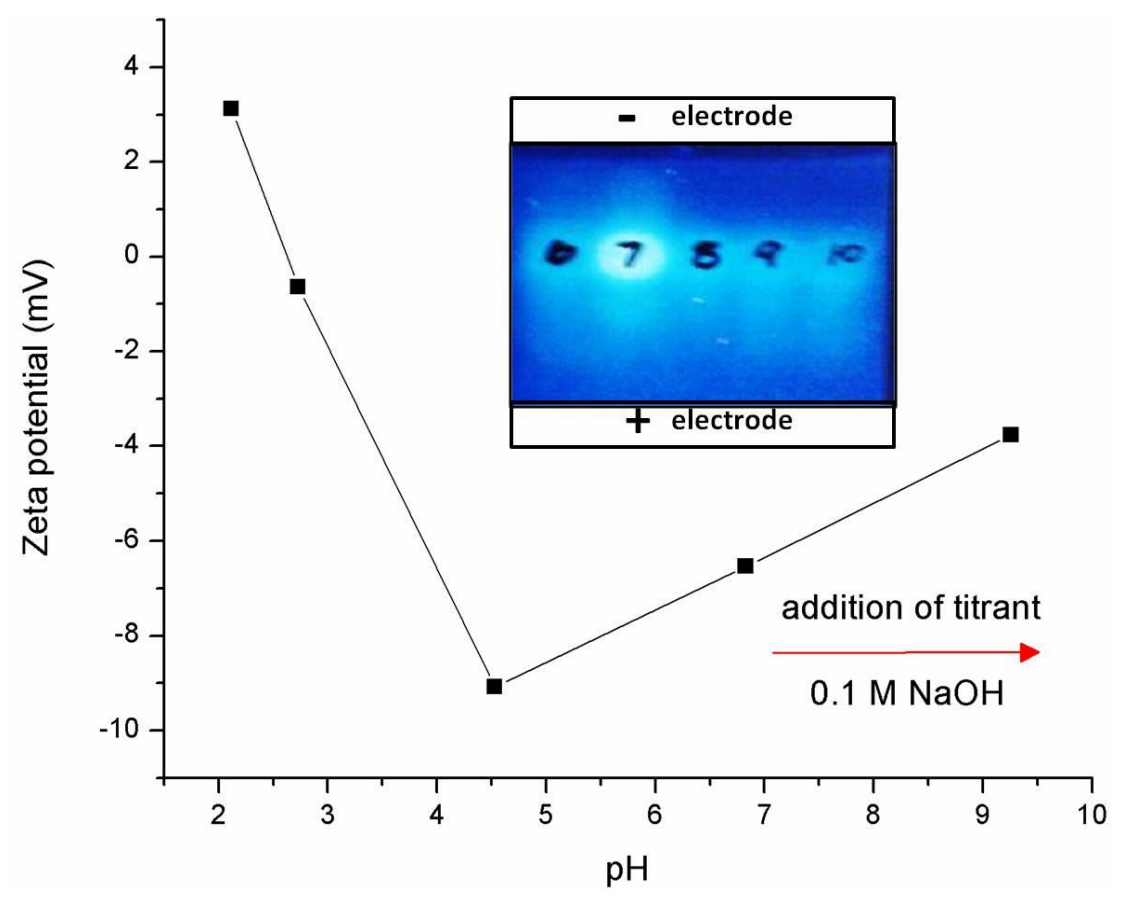

Figure 5.7. Zeta potential of the GA-synthesized as a function pH. Inset. Digital image of agarose gel under $302 \mathrm{~nm}$ light after electrophoresis of the luminescent Si NPs; wells 6-10 varying concentrations of (-) -charged GA-synthesized Si NPs. 
The photophysical properties of the hydrophilic, luminescent Si NPs from the one-step synthesis in GA were studied. Figure 5.8 shows the PL (a) and absorption spectra (inset) of the GA-synthesized Si NPs in water. Absorption spectra were collected on a series of dilutions of the Si NP solution, and on an aqueous solution of neat GA. Neat GA does not absorb in the visible, while the solution of Si NPs has an onset of absorbance at approximately $500 \mathrm{~nm}$ with a distinct shoulder that peaks at about $350 \mathrm{~nm}$. The long absorption tail is consistent with the indirect bandgap character of Si while the shoulder at $\sim 350 \mathrm{~nm}(3.5 \mathrm{eV})$ has been associated with Si direct bandgap transitions. ${ }^{[4,20 \mathrm{~d} \text {, }}$ 24] Both of these features have been observed in the absorption spectra for Si NPs of similar size. ${ }^{[4,20 \mathrm{~d}, 90 \mathrm{a}]}$

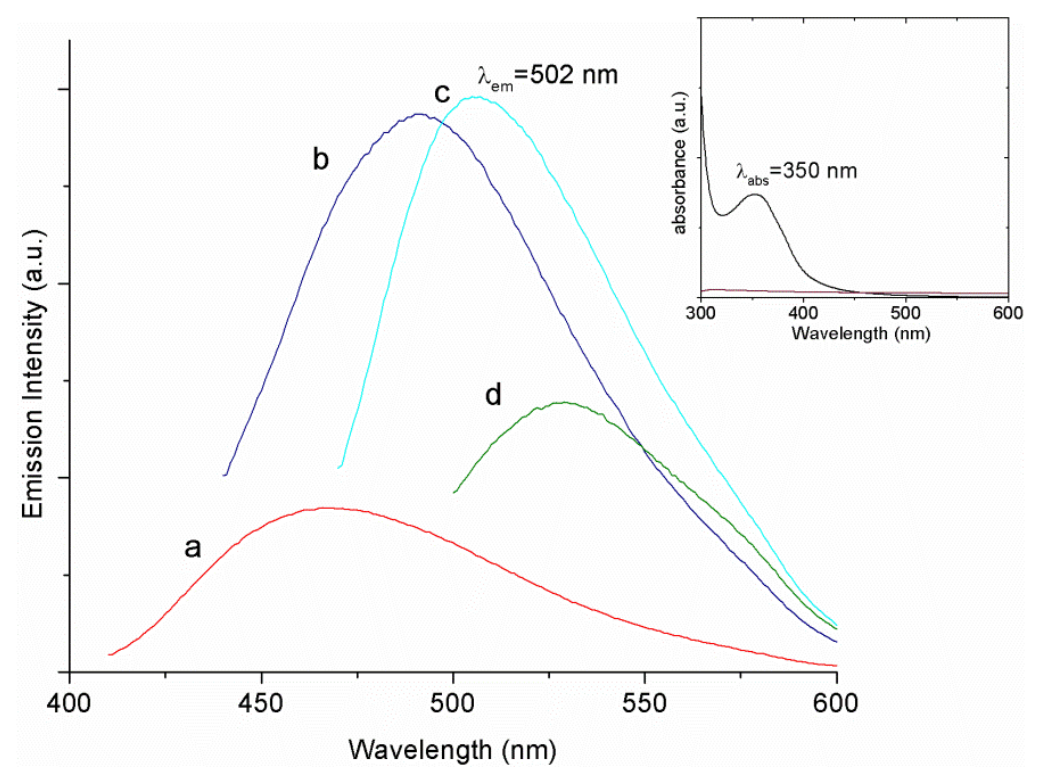

Figure 5.8. Emission spectra of GA-synthesized Si NPs in $\mathbf{H}_{2} \mathbf{O}$ excited at 400 (a, red), 430 (b, dark blue), 460 (c, light blue), and $490 \mathrm{~nm}$ (d, green); Inset. UV-Vis absorption spectra of neat GA (red) and Si NPs synthesized via the one-step melt synthesis in GA (black). 
Chapter 5: One -Step Zintl Salt Metathesis of Water-Soluble, Emissive Si NPs 97

The emission spectra of the as-prepared, aqueous Si NP colloid were collected using a series of excitation wavelengths ranging from $310-520 \mathrm{~nm}$ in $30 \mathrm{~nm}$ intervals. As a control, an aqueous sample of GA of similar concentration to that used in the synthesis was also measured and was not luminescent. The GA-synthesized Si NP solution has an emission maximum at $502 \mathrm{~nm}$ when excited at $460 \mathrm{~nm}$, and the excitation wavelength dependence of the emission observed for the sample is typical of previously reported blue-emitting Si NPs; ${ }^{[20 d, 97 a, 98,109 a, 123]}$ this spectroscopic behavior is generally attributed to size polydispersity and the excitation of different sized NPs with different excitation wavelengths. The QY of the GA-synthesized Si NPs, relative to 2-aminopyridine, was determined to be $13(1) \%$, which is comparable to the QYs for other Si NP colloids

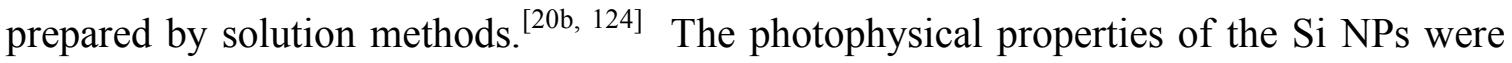
found to be stable over time, as spectroscopic measurements taken after several months of storage under ambient conditions were identical to those of freshly prepared samples (data not shown).

\subsubsection{Citric Acid (CA)- and ethylenediamine (en)-synthesized Si NP Characterization and Photophysical Properties}

Representative TEM images of the Si NPs prepared from the one-step synthesis in CA and en are shown in Figure 5.9 (A and B). These Si NPs were of similar size to those prepared in GA at $5.84 \pm 1.29 \mathrm{~nm}$ and $4.84 \pm 1.36 \mathrm{~nm}$, respectively (histograms in Appendix), suggesting that the one-step Zintl salt synthesis method consistently produces small, fairly monodisperse Si NPs. Though the average CA-synthesized Si NP is slightly larger than the accepted value for the Si Bohr radius, ${ }^{[92 \mathrm{a}]}$ these aqueous colloid solutions 
are still fluorescent (see below) HR-TEM of the CA- and en-synthesized Si NP cores possess lattice fringes, supporting the presence of $\mathrm{Si}^{0}$ cores. The fast Fourier Transform (FFT) of the Si NP images, shown in the insets of Figure 5.9, show multiple diffraction

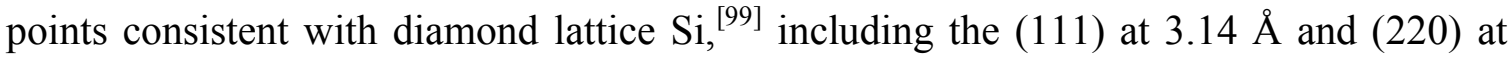
$1.92 \AA$ (JCPDS card no. 001-0787), similar to the diffraction pattern of the GAsynthesized Si NPs. Additional diffraction points at $3.4 \AA$ and $2.1 \AA$ (marked with *) are consistent with the diffraction patterns of the (011) and (111) planes of crystalline $\mathrm{SiO}_{2}$ (JCPDS card no. 087-2096.)
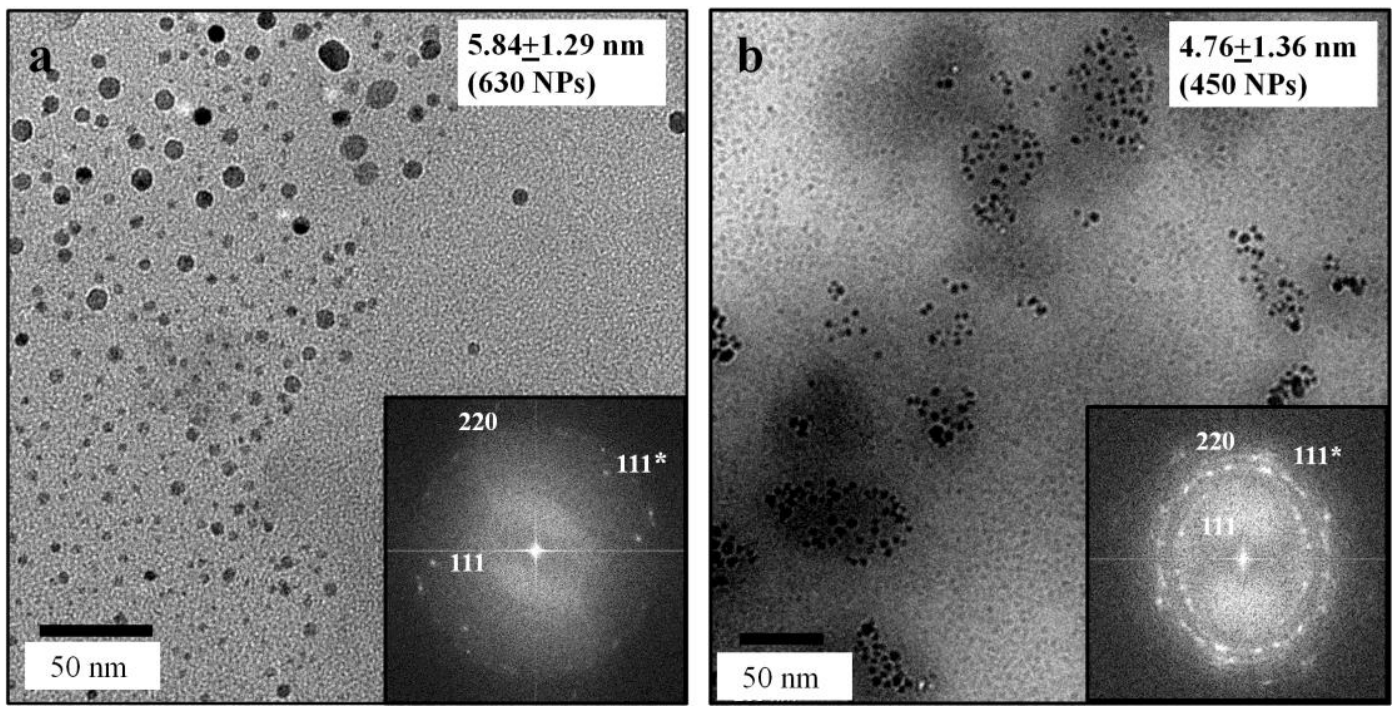

Figure 5.9. TEM images of (a) CA- and (b) en-synthesized Si NPs; Insets: FFT of the images of the Si $\mathrm{NP}$ cores reveal crystalline diffraction patterns, which are consistent with the presence of $\mathrm{Si}^{0}$ and $\mathrm{SiO}_{2}$.

The FT-IR spectra $\left(600-1800 \mathrm{~cm}^{-1}\right)$ of neat CA and purified CA-synthesized Si NPs are shown in Figure 5.10. Similar to the GA-synthesized Si NPs, it was anticipated that $\mathrm{CA}$ would covalently bond to the $\mathrm{Si}$ or $\mathrm{SiO}_{\mathrm{x}} \mathrm{NP}$ surface through at least one of its $\mathrm{COOH}$ groups, and therefore, the $\mathrm{C}=\mathrm{O}$ region can be monitored for environmental 
changes. Compared to neat $\mathrm{CA}$, the carbonyl region of the CA-synthesized Si NPs is broadened (at $1687 \mathrm{~cm}^{-1}$ ), and though not fully split, there appears to be a strong shoulder at $1631 \mathrm{~cm}^{-1}$. This represents a potential change in the CA carbonyl region (relative to neat CA) at 1741 and $1697 \mathrm{~cm}^{-1}$ (representing the protonated and deprotonated carboxylic forms, respectively). The red shifts of the carbonyl are similar to those observed in the GA-synthesized Si NPs, suggesting that CA may also interact with the Si NP surface in a bi-dentate manner. There is also a Si-O stretch and bend at $1063 \mathrm{~cm}^{-1}$ and $797 \mathrm{~cm}^{-1},{ }^{[121]}$ which may be due to the binding of CA to the surface, as well as surface oxidation from water. The other peaks from neat $\mathrm{CA}$ are not explicitly identifiable in the Si NP spectrum.

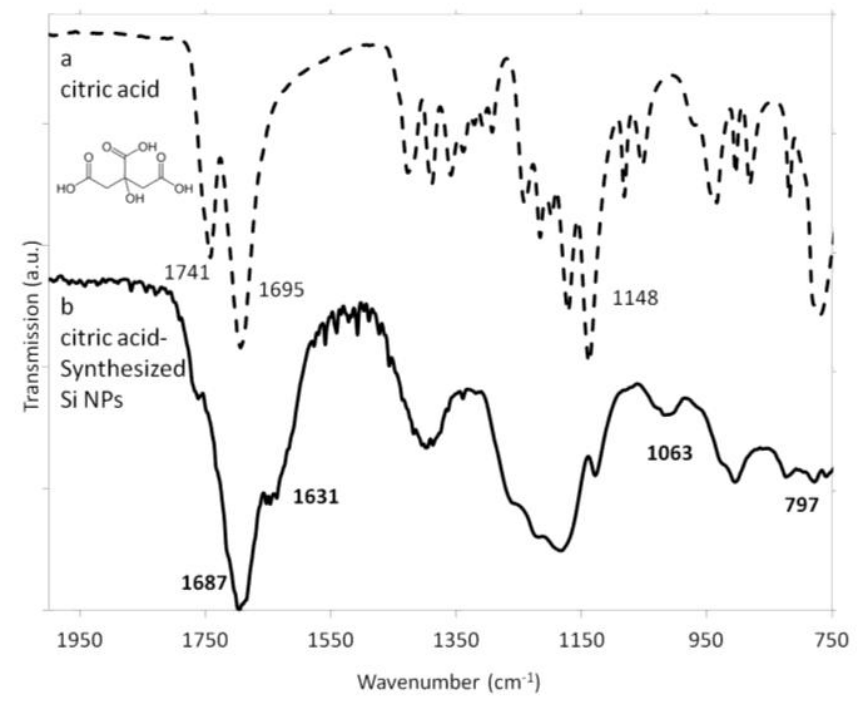

Figure 5.10. Fingerprint regions of the FT-IR spectra for (a) neat CA and (b) CA-synthesized Si NPs.

The FT-IR spectra (600-1800 $\left.\mathrm{cm}^{-1}\right)$ of neat en and purified, en-synthesized Si NPs are shown in Figure 5.11. Similar to the di-carboxylic acid ligands used to synthesize 
the Si NPs, it is anticipated that the amine groups may interact with the Si NP surface, datively or covalently, and thus changes in the N-H stretch could be monitored in the ensynthesized Si NP spectra. Neat en exhibits N-H stretches at $1591 \mathrm{~cm}^{-1}$ and $811 \mathrm{~cm}^{-1}$, while the en-synthesized Si NP spectra appears to have a red shift in this stretch to 1477 $\mathrm{cm}^{-1}$. Because less energetic N-H stretching could be present in the Si NPs, it is possible that this group could be interacting with the NP surface. Covalent Si-N stretches, ${ }^{[125]}$ consistent with en on the surface of the Si NPs, are expected ca. $800 \mathrm{~cm}^{-1}$. However in the en-synthesized Si NP spectrum, overlapping peaks from neat en make identification of Si-N stretches difficult. Additionally, there is a sharp peak at $1008 \mathrm{~cm}^{-1}$, and though it is sharper than the expected peak for $\mathrm{Si}-\mathrm{O}$, it could be related to the presence of surface oxidation. Therefore, strictly by FT-IR, it is difficult to ascertain the surface chemistry of the en-synthesized Si NPs.

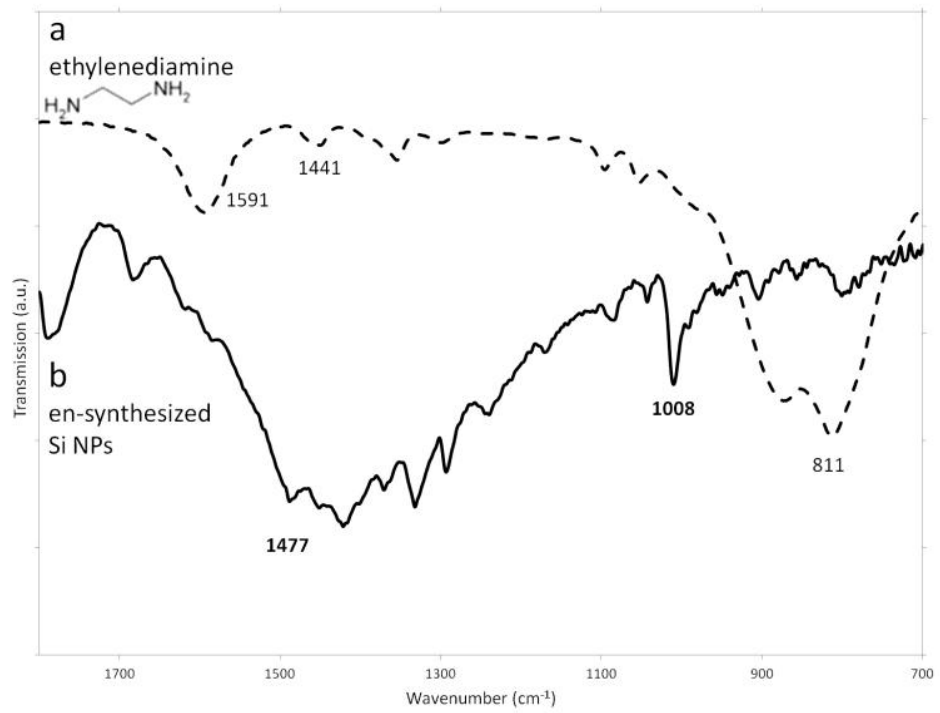

Figure 5.11. Fingerprint regions of the FT-IR spectra for (a) neat en and (b) en-synthesized Si NPs. 
Because the FT-IR results of the CA- and en-synthesized Si NPs did not make clear the potential solvent ligands and NP interaction, zeta potential measurements and gel electrophoresis of the Si NP samples were compared to examine the potential solvent molecule surface grafting. Zeta potential measurements were made on a series of aqueous CA-synthesized and en-synthesized Si NPs at varying $\mathrm{pH}$ values to compare the surface charge characteristics, indicative of the identity of surface bound species of Si NPs originating from different bi-functional solvents. As shown in Figure 5.12, Si NPs synthesized in CA exhibit a gradually increasing negative potential when titrated to higher $\mathrm{pH}$ due to the increasing partial deprotonation of $-\mathrm{COO}^{-}$groups at $\mathrm{pH}>\mathrm{pK}_{\mathrm{a}}$ $\left(\mathrm{pK}_{\mathrm{a}(1)}\right.$ of CA is 3.13). Conversely, the en-synthesized Si NPs have an increasing positive zeta potential when titrated to a lower $\mathrm{pH}$, which is consistent with the increasing number of charged $-\mathrm{NH}_{3}{ }^{+}$groups when $\mathrm{pH}<\mathrm{pK}_{\mathrm{a}}\left(\mathrm{pKa}_{(1)}\right.$ of en is 10.08). These results are consistent with the electrophoretic mobility results of Mahalingam et al., ${ }^{[126]}$ and indicate the ability of the one-step melt synthesis to chemically vary the Si NP surface charge via the variation in the identity of the ligand solvent. 
a

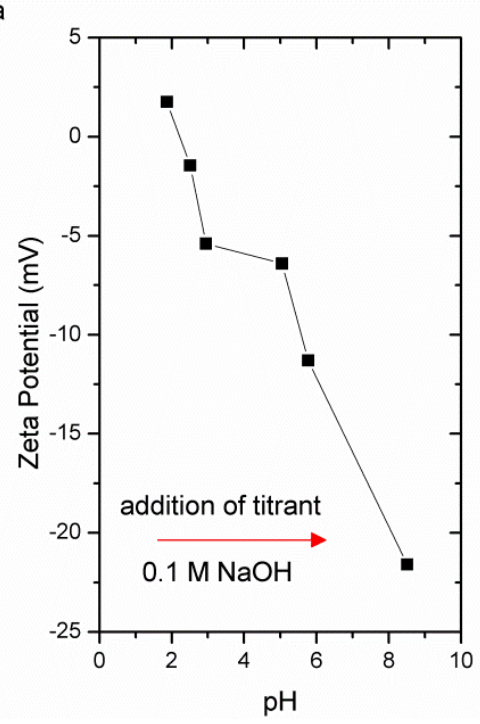

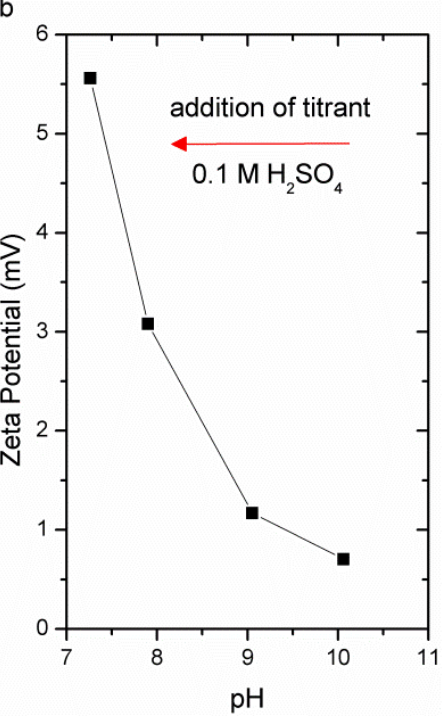

Figure 5.12. Zeta potential of the (a) CA-synthesized and (b) en-synthesized Si NPs as a function pH.

To corroborate the differing surface charges of the Si NPs, gel electrophoresis was performed. Figure 5.13 shows the migration of CA- and en-synthesized Si NPs to be consistent with the zeta potential results (at $\mathrm{pH}$ 8.3): CA-synthesized NPs migrate toward the positive electrode, suggesting that they are negatively charged (similar to the GA-synthesized Si NPs), while the en-synthesized NPs migrate toward the negative electrode, suggesting that they are positively charged. Along with the electrophoretic mobility behavior of the positively charged en-synthesized Si NPs, this supports that the hydrophilic Si NPs produced by the one step synthesis method do possess surface bound ligand solvent molecules. 


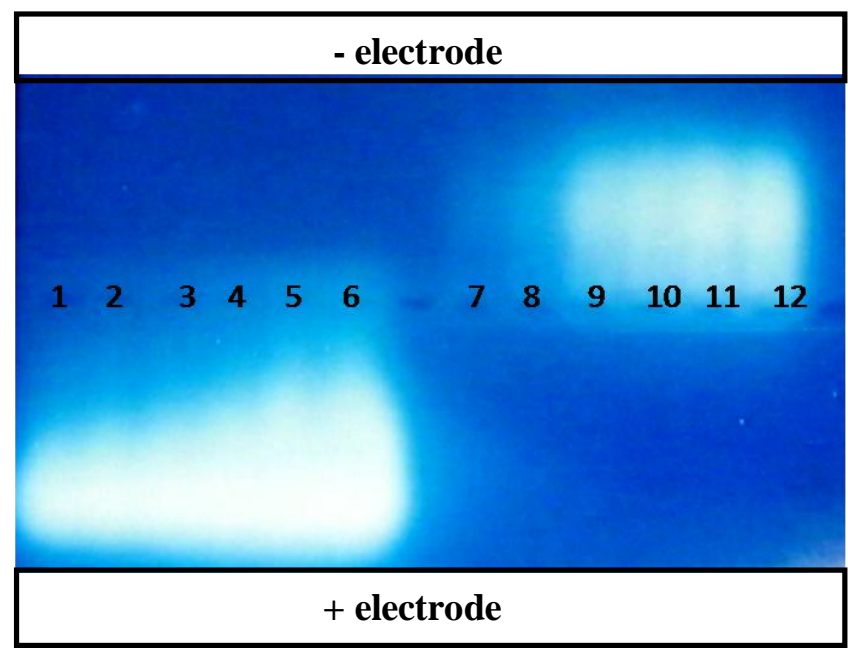

Figure 5.13. Digital image of agarose gel under $302 \mathrm{~nm}$ light after electrophoresis of the luminescent Si NPs; left to right: wells 1-6, increasing concentrations of (-)-charged CA-synthesized Si NPs; wells 7-12, increasing concentrations of (+)-charged en-synthesized Si NPs.

As observed in the agarose gel, the Si-NPs produced from the neat synthesis in neat $\mathrm{CA}$ and en were also fluorescent. Figure $\mathbf{5 . 1 4}$ shows the absorbance (line a) and the PL (lines b-e) of the CA- and en-synthesized Si NPs in water (A and B, respectively). The CA-synthesized Si NP solution has an onset of absorbance at approximately $400 \mathrm{~nm}$ with a strong at $260 \mathrm{~nm}$. The en-synthesized Si NPs have an onset of absorbance at about $550 \mathrm{~nm}$ with, what appears to be two peaks at 265 and $300 \mathrm{~nm}$. Both of the long absorption tails are consistent with the indirect bandgap character of Si. 

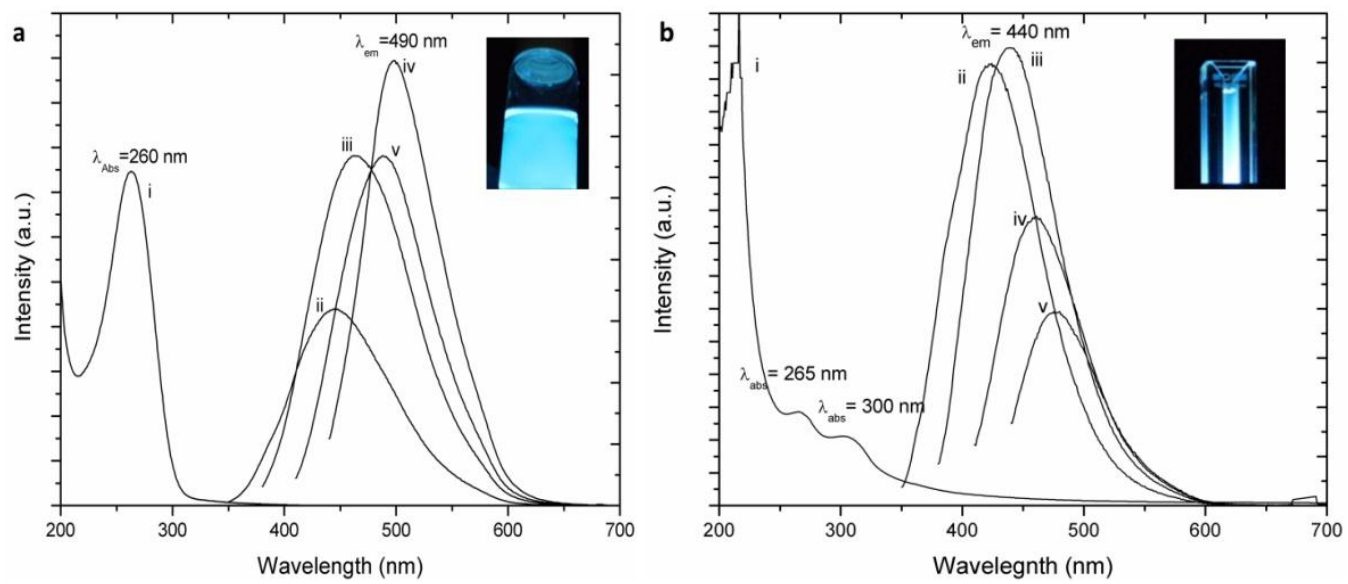

Figure 5.14. Absorbance (line i) and emission spectra (b) of neat, as-prepared (A) CA-synthesized, (B) en-synthesized Si NPs in $\mathrm{H}_{2} \mathrm{O}$ excited at (ii) $340 \mathrm{~nm}$, (iii) $370 \mathrm{~nm}$, (iv) $400 \mathrm{~nm}$, (v) and $430 \mathrm{~nm}$.

The CA- and en-synthesized Si NP solutions both have blue emission (observed via a handheld light, insets Figure 5.14). The CA-synthesized Si NPs have an emission maximum at $490 \mathrm{~nm}$ when excited at $430 \mathrm{~nm}$, while the en-synthesized Si NPs have an emission maximum of $440 \mathrm{~nm}$ when excited at $370 \mathrm{~nm}$. The average CA-synthesized $\mathrm{Si}$ NP was larger than the average en-synthesized Si NP $(5.48 \pm 1.29 \mathrm{~nm}$, vs. $4.84 \pm 1.36$ $\mathrm{nm}$, respectively), and as per the QC effect, require less energetic excitation, due to the larger bandgap. Therefore, it is possible to ascertain that the en-synthesized Si NPs are more quantum confined than the CA-Si NPs, requiring more energetic excitation $(370 \mathrm{~nm}$ vs. $430 \mathrm{~nm}$ ) to observe the maximum emission. Additionally, both samples exhibit the excitation wavelength dependence of the emission, consistent with a polydisperse size population.

Regardless of the medium used in the Zintl salt one-step metathesis, the QY values are similar. The CA-synthesized Si NPs were found to have a QY of $12 \%$ and the 
en-synthesized Si NPs were found to have 9\% (relative to 2-aminopyridine). Not only were the optical properties of the Si NPs found to be stable over several months, but the CA- and en-synthesized Si NP samples were found to be more concentrated than the GAsynthesized Si NPs; after purification, the CA- and en-synthesized Si NP solutions needed to be diluted, up to 10 -fold, to fully observe the emission maximum.

\subsection{Discussion of the Origin of Si photophysical properties}

The origin of emission from nanostructured $\mathrm{Si}$ is an ongoing discussion in the literature $^{[4,6 \mathrm{~d}, 22 \mathrm{c}, 24,99,116,127]}$ due to the possibility of radiative events originating from delocalized core states given that crystalline $\mathrm{Si}^{0}$ domains are present to generate exciton pairs (QC/LC), or from localized luminescent center (LC) states arising from surface, interface, or shell defects. ${ }^{[24,90 b, 91,93 d]}$ Further complicating the elucidation of the emission mechanism, Si NPs are readily oxidized, which results in $\mathrm{Si}^{0}$ core shrinkage and blue-shifting band-gap recombination pathways; ${ }^{[99]}$ as a result, the emission wavelength of nanosized $\mathrm{Si}$ is sensitive to sample age and processing. ${ }^{[90 \mathrm{~b}, 99]}$ Additionally, selftrapped excitons, as well as luminescent defect states, have been observed in pure $\mathrm{SiO}_{2}$ and silicon suboxides, ${ }^{[127 \mathrm{c}, 128]}$ and the possibility of these can complicate the assignment of luminescent states in heavily oxidized Si nanostructures, such as those in this work.

It has been reported that 4-5 $\mathrm{nm}$ Si particles, such as those produced by the onestep synthesis method, emit in the red region of the visible spectrum due to the electronhole recombination across the bandgap, consistent with expectations of QC. ${ }^{[93 \mathrm{~d}]}$ However, it has been determined both theoretically ${ }^{[97 b]}$ and experimentally ${ }^{[22 a]}$ that 
surface states, in addition to quantum-confined states, markedly influence Si NP optical properties, and therefore Si NPs in the QC regime embedded in oxide matrices have been reported to exhibit both a blue and a red emission event. ${ }^{[22]}$ It has been suggested that these co-existent emissions may be observed due to competing decay pathways of coregenerated excitons. ${ }^{[90 \mathrm{~b}, 93 \mathrm{a}, 129]}$ In these systems, the red emission is attributed to the QC, direct band-gap transitions, while the blue emission is commonly attributed to exciton recombination at localized defect states at the oxide interface or in the oxide shell (QC/LC). ${ }^{[22 a, 22 b, 90 b]}$ The Si NPs synthesized by the one-step melt method exhibit only an intense blue emission, suggesting that emission from defect states dominates the photophysical behavior, unless, due to high levels of oxidation, the Si NP cores are very small and the quantum-confinement related emission event is strongly blue-shifted. However, blue-green emission has also been observed from amorphous silica nanostructures lacking an $\mathrm{Si}^{0}$ component, but in order to observe this, excitation wavelengths shorter than $250 \mathrm{~nm}$ or short, time-pulsed excitations are necessary. ${ }^{[128]}$ These instrumental conditions were not used for routine spectroscopic experiments, suggesting that pure $\mathrm{SiO}_{\mathrm{x}}$-based electronic transitions are not responsible for the observed blue emission from the as-synthesized Si NPs. Thus, the blue luminescence observed from the Si NPs produced by the one-step synthesis method may be attributed to either the quantum-confined state of ultra-small $(\sim 1-2 \mathrm{~nm}) \mathrm{Si}$ nanocrystals, or to radiative recombination of core-generated excitons at surface or shell defects.

Because O-associated surfaces on Si nanocrystals (e.g., surface oxidation) have been reported to act as radiative centers for the exciton pairs to recombine with very short 
lifetimes (ps-ns), as opposed to longer lived excited states characteristic of direct (ns-ms) or indirect gap states ( $\mu \mathrm{s}-\mathrm{ms}),{ }^{[20 \mathrm{c}, 24,99,127 \mathrm{c}, 129]}$ the excited state lifetime of the blue emissive GA-synthesized Si NPs was examined. Previous reports have shown that when both red and blue emissions are observed for oxide-embedded Si NPs, the longer-lived red event has generally been attributed to QC while the shorter-lived blue event has been attributed to oxygen-centered luminescence from localized states, which act as recombination centers for core-generated excitons. ${ }^{[22 b, 90 b, 129]}$ The lifetime data of the GA-synthesized Si NPs (Figure 5.15) indicates a multi-exponential decay when excited at $375 \mathrm{~nm}$ and monitored at $455 \mathrm{~nm}$. Under these conditions, the intensity-weighted average lifetime was found to be $3.6 \mathrm{~ns}$, consistent with the time-scale of defect-related emission from small $(<3 \mathrm{~nm})$ Si nanocrystals in oxide matrices. ${ }^{[129]}$ However, it should be noted that as the physical dimensions of the nanocrystal are reduced, carrier recombination becomes more facile, resulting in shorter lifetimes, ${ }^{[22 b, 24]}$ and making it more difficult to discern differences between band-gap and defect transitions in smaller Si nanocrystals. Because analytical characterization of the GA-synthesized Si NPs support the existence of small $\mathrm{Si}^{0}$ domains in samples that are luminescent prior to water addition and extensive oxidation, and because the particles are, in general, no larger than $5 \mathrm{~nm}$ including the oxide shell, the blue, ns-lifetime luminescence we observe is also consistent with exciton decay from quantum confined states. Supportive of this, similar blue emission characteristics have been observed for other small Si nanoparticles with Oassociated surfaces, including alcohols ${ }^{[25 b]}$ and carboxylic acids, ${ }^{[20 a, 101]}$ and are attributed to quantum confinement. ${ }^{[20 c, 96]}$ However, because the lifetimes of direct gap and defect- 
related emissions are reported to be similar in very small Si nanocrystals, ${ }^{[22 b, 129]}$ the value of lifetime alone cannot be used to distinguish the two phenomena and the wavelength dependence of lifetime should also be considered.

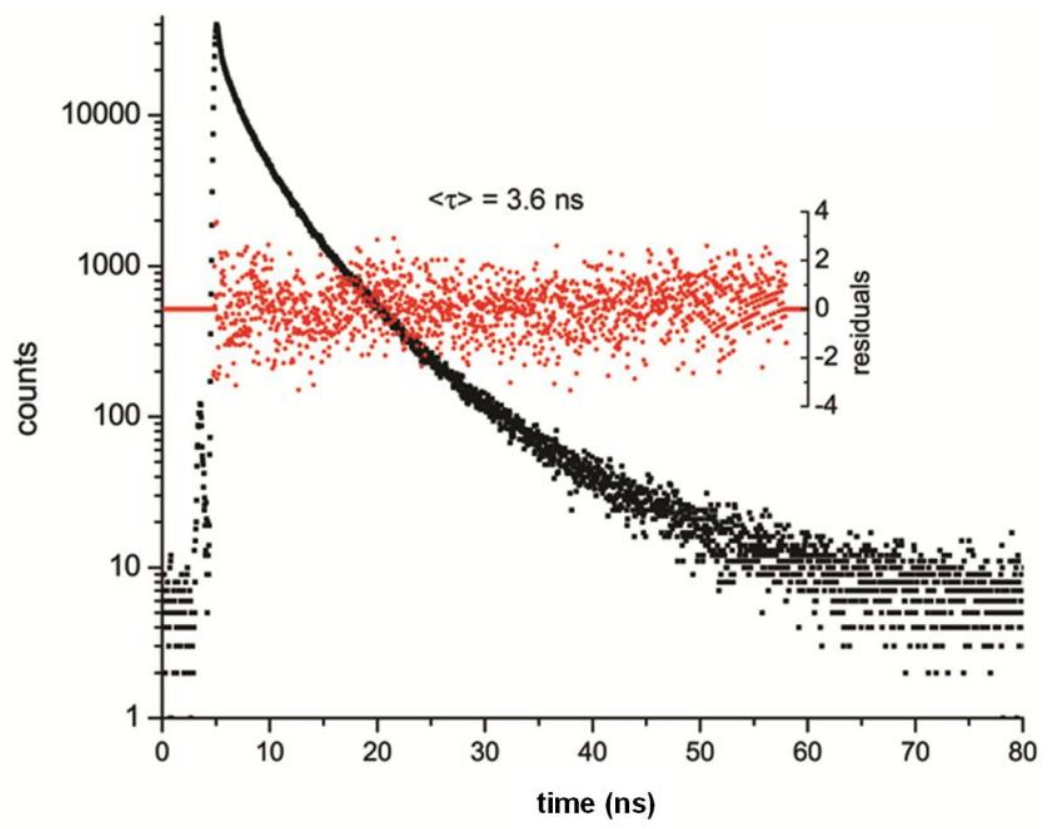

Figure 5.15. Time-resolved emission decay spectrum of GA-synthesized Si NPs excited at $375 \mathbf{~ n m}$. Emission was monitored at $455 \mathrm{~nm}$; the intensity-weighted lifetime was determined to be $3.6 \mathrm{~ns}$. The inset shows the residuals of the experimental curve fit, which results in a flat difference line.

When emission was monitored at other emission wavelengths (400 and $495 \mathrm{~nm}$; see Appendix), modest changes in lifetime were observed (intensity-weighted averages of 3.2 and $3.7 \mathrm{~ns}$, respectively); this slight wavelength dependence of lifetime may reflect the polydispersity in the Si NP size, as relaxation rates are understood to be a sizedependent property. Modest increases in lifetime with increasing emission wavelength are also observed in other polydisperse, oxidized silicon nanostructures, ${ }^{[20 c, 22 b]}$ and this 
behavior has been regarded as indicative of QC since little wavelength dependence is expected for luminescent, localized states that are discrete in nature. However, the lifetime changes with changing wavelength are small, and thus, cannot rule out exciton recombination at local states associated with the Si NP surface oxidation.

\subsection{Cellular Properties of GA-synthesized Si NPs}

Regardless of the origin of the observed luminescence, the exceptional stability of the aqueous, luminescent Si NPs synthesized by this one-step method indicates the high potential for their use as biomedical fluorophores, which has been examined in cellular uptake studies (Figure 5.16). The fast lifetimes of the water-soluble, highly luminescent Si NPs should be distinguishable from longer-lived cellular autofluorescence events, suggesting that these Si NPs may find utility as cellular imaging agents if they interact favorably in the cell environment. To investigate the affinity (or uptake) of the Si NPs toward a sample cell line, GA-synthesized Si NPs were incubated with live mouse neuroblastoma cells (N2a) and the emission intensity was monitored at $525 \mathrm{~nm}$ (excitation at $400+/-60 \mathrm{~nm}$ ). The control image (Figure 5.16) shows minimal luminescence (mostly due to endogenous flavins) from the $\mathrm{N} 2 \mathrm{a}$ cells relative to the $\mathrm{N} 2 \mathrm{a}$ cells with the incorporated Si NPs (Figure 5.16). Thus, the luminescence observed from the N2a cells arises from the Si NPs that have been taken up into the cells. The bright signal is distributed evenly throughout the $\mathrm{N} 2 \mathrm{a}$ cell, indicating that the negatively charged, luminescent particles were taken up into the cytoplasm with retention of their photophysical properties. Given that cellular uptake and distribution is dependent on 
charge, as well as motif recognition, the ability to modify the surface charge and functionality via our one-step synthesis method makes these Si NPs a versatile platform for development of more selective, targeted NP imaging agents in the future.
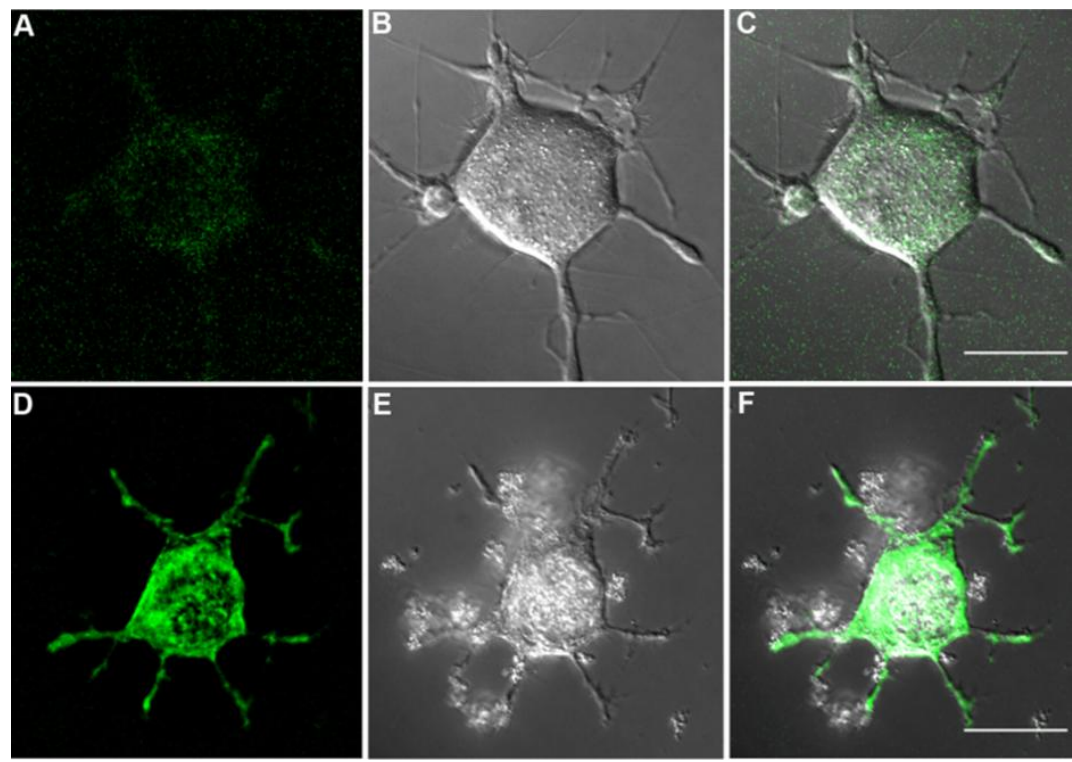

Figure 5.16. GA-synthesized Si NPs incubated with N2a cells to monitor cellular uptake. Fluorescence images of live N2a cells for control (a, no luminescent Si NPs added) and experimental (d, fluorescent Si NPs added) specimens. DIC images show the outline of the cell structure in control (b) and experimental (e) cells. Overlay of the fluorescence and optical images of control (c) and experimental (f) cells. Scale bars $=10 \mu \mathrm{m}$.

\subsection{Conclusions}

A versatile one-step, Zintl salt metathesis has been developed to produce watersoluble, photoluminescent Si nanoparticles (NPs), which despite the surface oxidation, bear at least some surface-bound bi-functional solvent molecules, including glutaric acid (GA), citric acid (CA), and ethylenediamine (en). TEM studies indicate that this one-step method produces small, monodisperse NPs with little dependence on the identity of the 
ligand solvent. The diffraction patterns reveal that these particles contain nanoscale $\mathrm{Si}^{0}$ domains, which have been additionally confirmed by Raman spectroscopy and Powder X-ray Diffraction. All particles synthesized via the one-step synthesis Zintl salt metathesis exhibit a bright photoluminescence in the blue region of the visible spectrum with emission lifetime decays 3-4 ns. While the emission mechanism of these particles has not yet been confirmed, the ease and versatility of synthesis, the long-term aqueous stability of the NP products, and the extreme robustness of the photophysical properties make these Si NPs excellent candidates for biomedical applications, as demonstrated by the imaging of the GA-synthesized NPs in N2a cells. 


\title{
Using Silicon Nanoparticles as Reducing Agents for Metal Ions
}

\begin{abstract}
The quenching behavior of blue- (glutaric acid-synthesized Si NPs, GA-Si NPs) and red$\left(\mathrm{SiO}_{\mathrm{x}} / \mathrm{H}_{\mathrm{y}}-\mathrm{Si} \mathrm{NPs}, \mathrm{SiO}_{\mathrm{x}}-\mathrm{Si} \mathrm{NPs}\right)$ emitting $\mathrm{Si}$ NPs were investigated to gain insight on their reduction potentials by exposing them to metal salts. This interaction affected, not only the emission intensity of the Si NPs, but also the absorption spectra of the metal ions. The GA-Si NPs were found to reduce $\mathrm{AuCl}_{4}{ }^{-}$and $\mathrm{Ag}^{+}$ions, while the $\mathrm{SiO}_{\mathrm{x}}-\mathrm{Si} \mathrm{NPs}$ were only able to reduce $\mathrm{AuCl}_{4}{ }^{-}$. The reduction of $\mathrm{AuCl}_{4}{ }^{-}$is expected to be spontaneous in the presence of crystalline $\mathrm{Si}$, and explains why both crystalline $\mathrm{Si}$ NP systems produced $\mathrm{Au}$ NPs from the addition of $\mathrm{HAuCl}_{4}$. However, crystalline $\mathrm{Si}$ is not expected to reduce $\mathrm{Ag}^{+}$. The GA-Si NP emission may originate from oxide embedded 1-2 $\mathrm{nm}$ Si crystals in accordance with the quantum confinement (QC) effect, which appears to also affect the reduction potential of Si, promoting the spontaneity of the GA-Si NP photo-reduction of $\mathrm{Ag}^{+}$to $\mathrm{Ag}$ NPs. The $\mathrm{SiO}_{\mathrm{x}}-\mathrm{Si} \mathrm{NPs}$, which did not reduce $\mathrm{Ag}^{+}$, may have a reduction potential closer to that of bulk Si $(-0.84 \mathrm{~V})$.
\end{abstract}

In preparation for submission 


\subsection{Introduction}

In recent years, emission from porous and colloidal nanosized silicon ( $\mathrm{Si}$ ) has attracted a great deal of attention due to their unique optical, electrical, and chemical properties, with the ultimate goal of understanding and utilizing these properties. ${ }^{[18 a, 130]}$ Due to the quantum confinement (QC) effect, Si nanoparticles (NPs) have been reported to exhibit size-tunable photoluminescence (PL), which could be employed for biological tagging or imaging, as well as other light emission applications. ${ }^{[6 \mathrm{~d}, 91,131]}$ Nonetheless, the emission mechanism from porous and nanosized Si has been debated. Originally, it was believed that Si emission originated from QC from exciton annihilation across the bandgap. However, it was later determined that the recombination of excitons formed within the Si core can also occur at surface defect sites or dangling bonds. ${ }^{\text {[6d, 24, 90a, 116, 127a] }}$ Therefore, in addition to NP size, the surface chemistry and surrounding media have been found to affect the emission properties, particularly emission wavelength, from nanosized Si.

In trying to understand the origin of visible light emission from nanocrystalline $\mathrm{Si}$, many have reported the observation of the quenching, or photodegradation, of nanosized Si emission intensity. Most of these observations have involved quenching the emission from porous silicon via the addition of various organic solvents, ${ }^{[132]}$ acids and bases, ${ }^{[133]}$ amines, ${ }^{[134]}$ aromatic nitro compounds, ${ }^{[134 a, 135]}$ and metal oxides, ${ }^{[136]}$ such as $\mathrm{WO}_{3}$. Particularly, Andsager et al. described porous Si emission quenching by various metals ions, which were deposited on a porous $\mathrm{Si}$ surface, ${ }^{[137]}$ the $\mathrm{Si}$ emission was quenched upon the deposition of $\mathrm{Au}^{3+}, \mathrm{Ag}^{+}$, and $\mathrm{Cu}^{2+}$, but not noticeably by other metals. 
While they did not assign a definitive mechanism to the quenching, they stated that the two likely causes are that the adsorbed metals could be interacting with the incident or emitted photons, or that the adsorbed metals maybe disrupting the emission mechanism.

While there are a number of published studies on the emission quenching of porous $\mathrm{Si}$, relatively few studies have addressed the emission quenching of Si NPs. Similar to the quenching of porous Si, freestanding colloidal Si NPs have been exposed to amines ${ }^{[73,134 a, 138]}$ or other molecules ${ }^{[136,139]}$ and ions ${ }^{[140]}$ to determine the effect of solution additives on the observed emission intensity. Germankeno et al. performed a very elegant study in which they exposed Si NPs to aromatic nitro molecules and monitored the emission and excited state lifetimes. ${ }^{[73]}$ They found that the molecules whose reduction potentials lie below the approximate edge of the bulk $\mathrm{Si}$ conduction band were able to quench the emission from red-emitting Si nanocrystals. The cause of this $\mathrm{Si}$ NP quenching was believed to originate from the electron transfer from the $\mathrm{Si}$ conduction band to the vacant orbitals of the quenchers.

The Germankeno et al. study revealed that Si NPs are able to undergo oxidation/reduction reactions. However, very few other studies have utilized this observation to gain insight on the origin of Si NP emission. Presented herein is an optical study (using UV-Vis and photoluminescence spectroscopies) aimed at observing the reduction behavior of blue-emitting and red-emitting Si NPs in the presence of metal ions. Based on the excitation wavelength dependence on the emission, which varies between the two Si NP samples, suggests the NP systems may possess different emission mechanisms. The blue-emitting Si NPs, coated in glutaric acid $(\mathrm{GA}),{ }^{[25 a]}$ exhibit an 
excitation wavelength dependent emission maximum, indicative of QC, while the redemitting Si NPs, possessing a mixed oxide/hydride surface termination, do not. ${ }^{[3 c],[141]}$ After exposing the $\mathrm{Si} \mathrm{NP}$ solutions to $\mathrm{AuCl}_{4}{ }^{-}$and $\mathrm{Ag}^{+}$ions, the emission intensity of the Si NPs decreased, and the formation of metal NPs occurred. The formation of metal NPs lends insight into the origin of the emission of the two Si NP systems.

\subsection{Experimental}

\subsubsection{Synthesis of Si NPs for Photophysical Studies}

The first photophysical study uses blue-emitting glutaric acid (GA)-synthesized Si NPs prepared from a previously reported one-step melt synthesis. ${ }^{[25 a]}$ Briefly, NaSi, $\mathrm{NH}_{4} \mathrm{Br}$, and solid GA (in a 1:2:10 stoichiometric ratio) were loaded into a two-neck round-bottomed flask in a $\mathrm{N}_{2}$-filled glovebox. Upon transfer to an Ar-atmosphere Schlenk line, the mixture was heated to melt GA $\left(98{ }^{\circ} \mathrm{C}\right)$, and then refluxed $\left(200{ }^{\circ} \mathrm{C}\right)$ for $24 \mathrm{hrs}$. Upon cooling, nanopure water $\left(\mathrm{nH}_{2} \mathrm{O}, 18 \mathrm{MOhm} \cdot \mathrm{cm}\right.$ resistivity) was added to the mixture, and was stirred for 2 hrs. Black solids were removed by air-free vacuum filtration, and subsequently identified as bulk Si. The solution of GA-synthesized Si NPs was purified using sublimation and dialysis. The obtained, purified NPs ("GA-Si NPs") were taken to dryness and subsequently and re-dispersed in $50 \mathrm{~mL} \mathrm{nH}_{2} \mathrm{O}$.

The second photophysical study uses red-emitting Si NPs prepared from the controlled oxidation of hydride-terminated Si NPs. ${ }^{[3 \mathrm{c}]}$ Briefly, trichlorosilane $\left(\mathrm{HSiCl}_{3}\right.$, Alfa Aesar, 97\%) was loaded into a two-neck round-bottomed flask on the Schlenk line, and cooled to $0{ }^{\circ} \mathrm{C}$, at which time, $90 \mathrm{mmol}$ of $\mathrm{nH}_{2} \mathrm{O}$ was rapidly injected to catalyze the 
polymerization of $\mathrm{HSiCl}_{3}$. The polymer was then annealed at $1100{ }^{\circ} \mathrm{C}$ under flowing $\mathrm{N}_{2}$ in a tube furnace (Lindberg blue Mini-Mite) for $10 \mathrm{hrs}^{[141]}$ The nanocrystalline Si, encapsulated in a $\mathrm{SiO}_{2}$ matrix, were ball-milled for $10 \mathrm{~s}$ using a high-energy mill mixer (Spex 8000M), and etched using concentrated hydrofluoric acid (aq. 47-52\%), ethanol, and water (volume 1:1:1). The chemical etch was quenched after $1 \mathrm{hr}$ by extracting the hydride-terminated Si NPs into pentanes. To perform the controlled oxidation, $6 \mathrm{~mL}$ of Si-H NPs were added to a 50:50 mixture of ethanol:water and $1 \mathrm{~mL}$ of $30 \%$ (aq.) $\mathrm{H}_{2} \mathrm{O}_{2}$, and the mixture was left stirring for 48 hrs. The $\mathrm{Si} / \mathrm{SiH}_{\mathrm{y}} \mathrm{O}_{\mathrm{x}}$ core/shell NPs (" $\mathrm{SiO} \mathrm{x}_{\mathrm{x}}-\mathrm{Si}$ NPs") were taken to dryness, and subsequently dispersed in $10 \mathrm{~mL}$ of $\mathrm{nH}_{2} \mathrm{O}$.

\subsubsection{Photophysical Study using Si NPs and Metal Ions}

The emission spectra of the neat Si NPs were collected under ambient conditions, as well as after UV exposure from a handheld UV lamp (6 W, $365 \mathrm{~nm})$. For consistency, the GA-Si NP solution was diluted until the maximum UV-Vis absorbance was approximately 1.0 , which was a $1 \%$ dilution of the neat GA-Si NP solution. Because the absorption and emission of the $\mathrm{SiO}_{\mathrm{x}}-\mathrm{Si}$ NPs was less intensity, the aqueous solution of the $\mathrm{SiO}_{\mathrm{x}}-\mathrm{Si}$ NPs was used as prepared.

All photophysical experiments were carried out in $\mathrm{nH}_{2} \mathrm{O}(\mathrm{pH}=6.3)$. UV-Vis absorption spectra $(190-700 \mathrm{~nm})$ were collected on a Shimadzu Model UV-2450 spectrophotometer. Steady-state photoluminescence (PL) spectra were collected on a Shimadzu-RF5301PC spectrophotometer using an excitation wavelength of $350 \mathrm{~nm}$ and the emission was monitored from 360-700 nm. 
The absorption and emission spectra of the Si NPs were also collected in the presence of metal ions. Si NP aliquots $(0.8 \mathrm{~mL})$ were added to $0.2 \mathrm{~mL}$ tetrachloroauric acid $\left(\mathrm{HAuCl}_{4}\right.$, Fisher Scientific) or silver nitrate $\left(\mathrm{AgNO}_{3}\right.$, Fisher Scientific, $\left.98.5 \%\right)$ in various concentrations $(0.1-10 \mathrm{mM})$ in a standard, quartz $1-\mathrm{cm}$ cuvette. The UV-Vis and emission spectra of the Si NP control and Si NP + metal ion solutions were collected under ambient conditions, or after UV irradiation using a handheld UV lamp for various times.

\subsubsection{Analytical Characterization of the Si NPs + Metal Ion Solutions}

Transmission Electron Microscopy (TEM) and Energy Dispersive X-ray spectroscopy (EDX), performed on a Tecnai F-20 HR-TEM operating at $200 \mathrm{kV}$, were used to assess the size, size distribution, and composition of the as-prepared Si NPs + the metal NP products of Si NP photo-reduction. Topological EDX spectra for composition analysis were collected on isolated particles for 10 min with approximately $20 \%$ dead time between successive scans. Samples were prepared by drop casting aliquots of the samples onto 400-mesh holey carbon-coated copper grids (SPI), which were dried in air at $100{ }^{\circ} \mathrm{C}$ overnight. X-ray Diffraction (XRD) measurements were made on a Rigaku Ultima IV X-ray diffraction system using a thin film stage and scanning over the $20-50^{\circ}$ 2-theta angular range. The products from the GA-Si NPs $+\mathrm{AuCl}_{4}{ }^{-}$photochemical reaction were prepared by drop casting the solution onto glass slides to deposit a film. 


\subsection{Photochemical Reactions of the blue-emitting GA-Si NPs with Various Metal Ions}

\subsubsection{Characterization of the GA-Si NPs}

The GA-Si NPs used in this study were prepared by the one-step metathesis of the Zintl salt sodium silicide with ammonium bromide in the presence of glutaric acid (GA). As previously described, the GA-Si NPs were characterized by a number of techniques, including TEM, SAED, XPS, Raman, and XRD. ${ }^{[25 a]}$ The GA-Si NPs were found to have crystalline Si NP cores, which were oxide embedded with an average diameter of $3.9 \pm$ $0.8 \mathrm{~nm}$. The GA solvent ligand was believed to interact with the Si NP surface in a linear, bi-dendate manner, leaving one of the $\mathrm{COOH}$ groups free in solution. Surface features were also previously analyzed using FT-IR spectroscopy, zeta potential measurements, and gel electrophoresis migration. Additionally, according to the XPS and FT-IR results, a silicon suboxide shell $\left(\mathrm{SiO}_{\mathrm{x}}\right.$, where $\left.\mathrm{x}<2\right)$ was found to grow on the surface of the GA-NPs over time.

\subsubsection{Photostability of the neat GA-Si NPs}

The absorption and emission spectra of the diluted GA-Si NPs is shown in Figure

6.1. The absorption spectrum of the GA-Si NPs (line a) shows an absorption edge around $450 \mathrm{~nm}$ and continuous absorbance into the UV region, with a small shoulder at $290 \mathrm{~nm}$ (4.27 eV). The diluted GA-Si NPs have an emission maximum at $420 \mathrm{~nm}$, when excited at $350 \mathrm{~nm}$ (Figure 6.1, lines b). Even after the formation of a silicon suboxide layer, which occurs gradually over several months, the emission intensity and emission maximum show little change (data not shown). 
The neat, diluted GA-Si NP solution was also exposed to UV irradiation. The absorbance spectrum of the GA-Si NPs after 60 mins of UV irradiation looks identical to the $\mathrm{t}=0$ absorption spectrum and $96 \%$ of the original emission intensity (Figure 6.1 , inset) is preserved after the light treatment. The preservation of the emission characteristics of the GA-Si NPs over several months under ambient conditions, and under UV light for 60 mins, highlights the exceptional photostability of the as-prepared, blue-emitting GA-Si NPs.

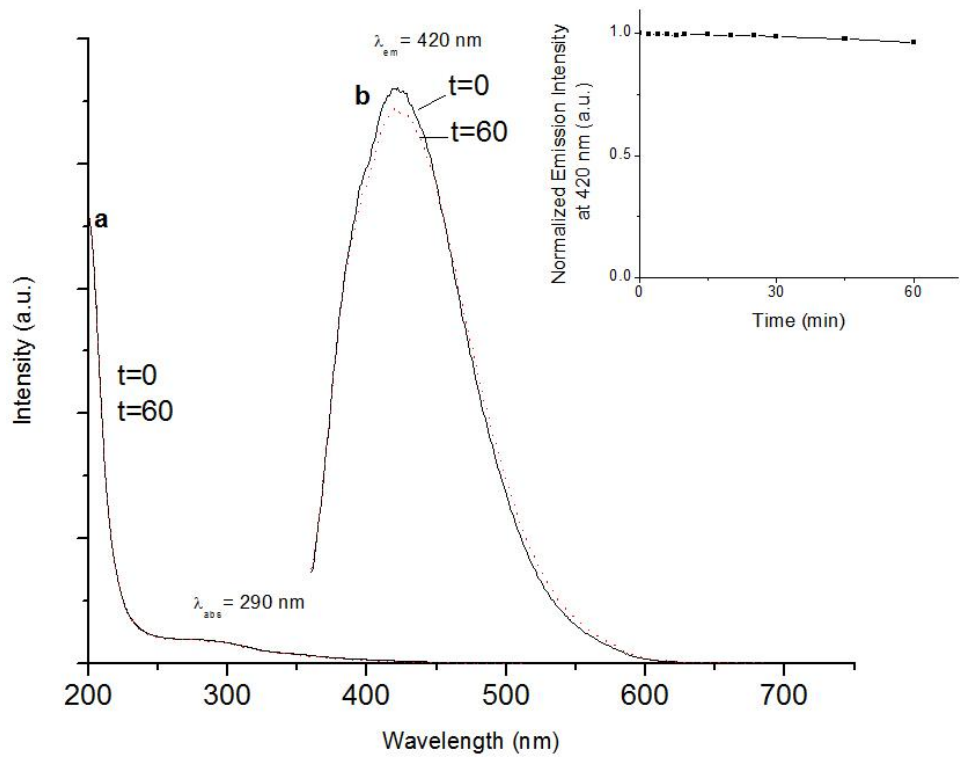

Figure 6.1. Optical properties of the neat, diluted GA-Si NPs. The absorbance spectra of the GA-Si NPs at $\mathrm{t}=0$ (black solid) and after 60 mins of UV irradiation (red dotted) are identical (lines a). The emission intensity of the GA-Si NPs after 60 mins of UV irradiation was $96 \%$ of the emission intensity at $\mathrm{t}=0$; Inset. The normalized emission intensity at $420 \mathrm{~nm}$ of the GA-Si NPs versus UV irradiation time.

\subsubsection{Photochemical Reaction of the GA-Si NPs with $\mathrm{AuCl}_{4}{ }^{-}$}

The absorption spectrum of neat $\mathrm{HAuCl}_{4}$ is shown in Figure 6.2 (line a) with an absorption peak at $290 \mathrm{~nm}$. This peak, along with the partially omitted peak at $225 \mathrm{~nm}$, 
originates from the ligand-metal charge transfers of $\mathrm{AuCl}_{4}{ }^{-}$. The absorbance spectra of the GA-Si NPs $+10 \mathrm{mM} \mathrm{HAuCl}_{4}$ solution are also shown in Figure 6.2 (lines b-g). After 30 mins of UV irradiation, no changes were observed in the GA-Si $\mathrm{NP}+\mathrm{HAuCl}_{4}$ solution absorption spectra (line c). However, after 60 mins, an absorption peak forms at $520 \mathrm{~nm}$ (line d); the solution also changed from light yellow to purple in color (Figure 6.2, inset). The emerged peak red shifted to $540 \mathrm{~nm}$ with increasing intensity with increasing UV irradiation time (up to $240 \mathrm{~min}$, line g). This peak is likely due to the surface plasmon resonance (SPR) of Au NPs, ${ }^{[142]}$ which is known to slightly red shifts with increasing particle size. ${ }^{[142 c, 143]}$

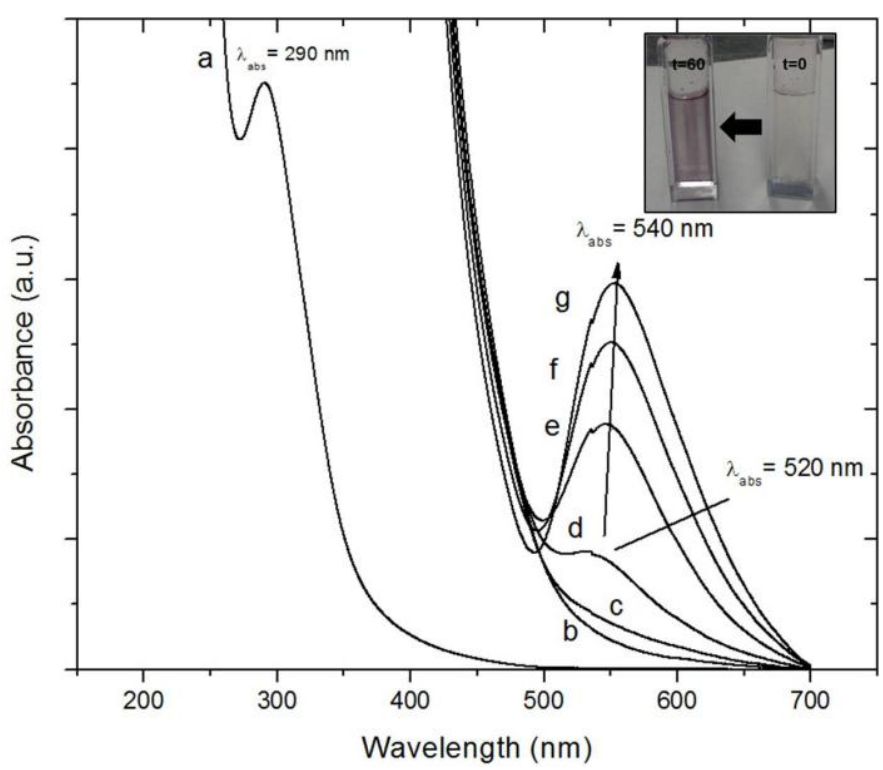

Figure 6.2. Absorption spectra of neat $\mathrm{HAuCl}_{4}$ and the GA-Si NPs $+\mathrm{HAuCl}_{4}$ photochemical reaction.

The neat $\mathrm{HAuCl}_{4}$ absorption spectra (line a) shows a small peak at $290 \mathrm{~nm}$. The GA-Si NPs and $\mathrm{HAuCl}_{4}$ were irradiated with UV light for various times: line $b, t=10$ mins, line $c, t=30$ mins, line $d$, $t=60$ mins, line $\mathrm{e}, \mathrm{t}=125 \mathrm{mins}$, line $\mathrm{f}, \mathrm{t}=180 \mathrm{mins}$, line $\mathrm{g}, \mathrm{t}=240 \mathrm{mins}$, The peak emerging at $520-540 \mathrm{~nm}$ is consistent with the SPR of Au NPs; Inset. Digital image of the GA-Si NP and $\mathrm{HAuCl}_{4}$ solution after 60 mins. 
As illustrated in Figure 2.1, the $\mathrm{CB}$ and VB of bulk metals are essentially continuous in energy, allowing electrons to move more freely through the solid by moving between the overlapping orbitals on light or heat excitation. When nanosized, metal particles are present, light can interact in a different way with the metal NP, as compared to the bulk metal: the electric field of an incident light wave can induce a charge separation within these particles. When the frequency of the field becomes coherent with the oscillation of the electrons, a strong absorption occurs, resulting in the SPR band. ${ }^{[142 c, 143]}$ Because this absorption occurs on the surface, the particle size, shape, and chemical surroundings, including the dielectric constants of the solvent, influence the intensity and position of the SPR. ${ }^{[142]}$

The emission spectrum of the GA-Si NPs in the presence of $\mathrm{HAuCl}_{4}$ was also monitored with increased UV exposure time (Figure 6.3). The emission maximum (420 $\left.\mathrm{nm}, \lambda_{\mathrm{ex}}=350 \mathrm{~nm}\right)$ was found to decrease significantly over time; the emission intensity decreased to $36 \%$ and $13 \%$ of the original emission after 30 mins and 240 mins of UV irradiation in the presence of $\mathrm{AuCl}_{4}{ }^{-}$, respectively. A slight blue shift in the emission wavelength $(10 \mathrm{~nm})$ is also observed, which may due to a small decrease in the size of the crystalline Si NP. 


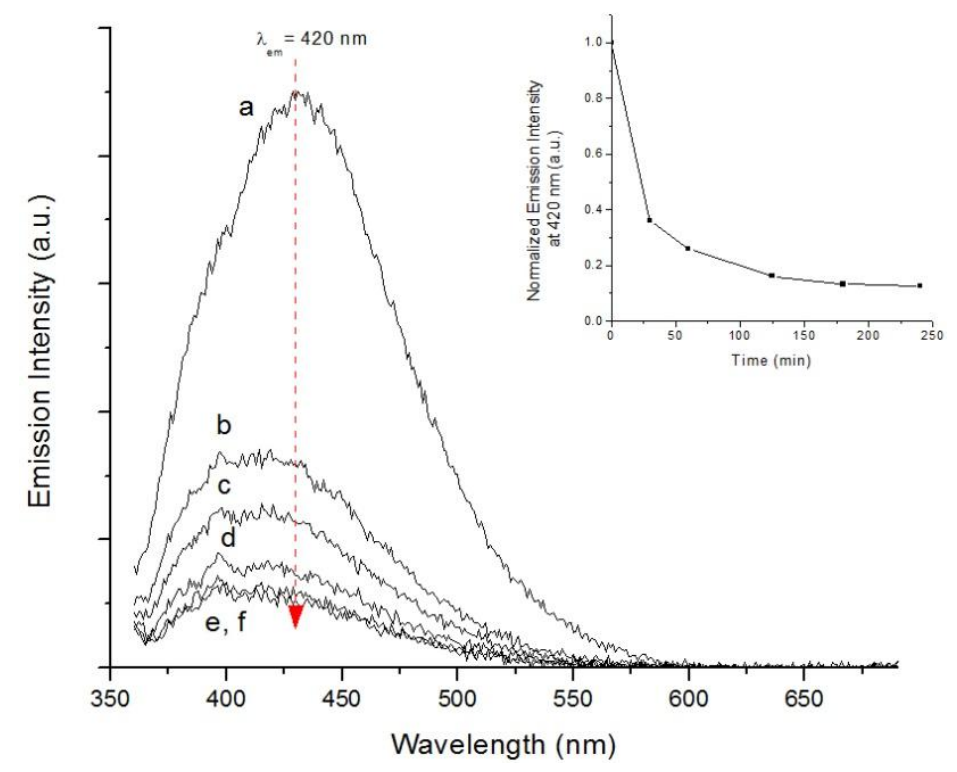

Figure 6.3. Emission spectra for the GA-Si NP $+\mathbf{H A u C l}_{4}$ photochemical reaction. The emission maximum of the GA-Si NPs $(420 \mathrm{~nm})$ in the presence of $10 \mathrm{mM} \mathrm{HAuCl}_{4}$ decreases with increasing UV irradiation time: line $\mathrm{a}, \mathrm{t}=0 \mathrm{~min}$, line $\mathrm{b}, \mathrm{t}=30 \mathrm{~min}$, line $\mathrm{c}, \mathrm{t}=60 \mathrm{~min}$, line $\mathrm{d}, \mathrm{t}=125 \mathrm{~min}$, line $\mathrm{e}, \mathrm{t}=180 \mathrm{~min}$, line $\mathrm{f}, \mathrm{t}=240 \mathrm{~min}$. Inset: The normalized emission intensity of the GA-Si NPs at $420 \mathrm{~nm}$ versus UV irradiation time.

The presence of the SPR band from the GA-Si NP $+\mathrm{HAuCl}_{4}$ solution indicated that Au NPs were forming in solution, and TEM was used to verify this. The TEM image of the GA-Si NP $+\mathrm{HAuCl}_{4}$ solution shows many spherical particles (Figure. 6.4A). The high contrast of these NPs indicates that they consist of high atomic weight elements. HR-TEM revealed crystalline particles, with visible lattice fringes and many faceted nanocrystals (Figure 6.4B); the FFT of the image of the NPs reveals diffraction reflections consistent with the lattice planes of $\mathrm{Au}$, including the (111) at $2.35 \AA$, (200) at $2.03 \AA,(220)$ at $1.43 \AA$, and (311) at $1.23 \AA$ (JCPDS card no. 001-1172, Figure 6.4C). 
The average diameter of the Au NPs was found to be $21.55 \pm 10.13 \mathrm{~nm}$ after counting 200 NPs (Figure 6.4D).

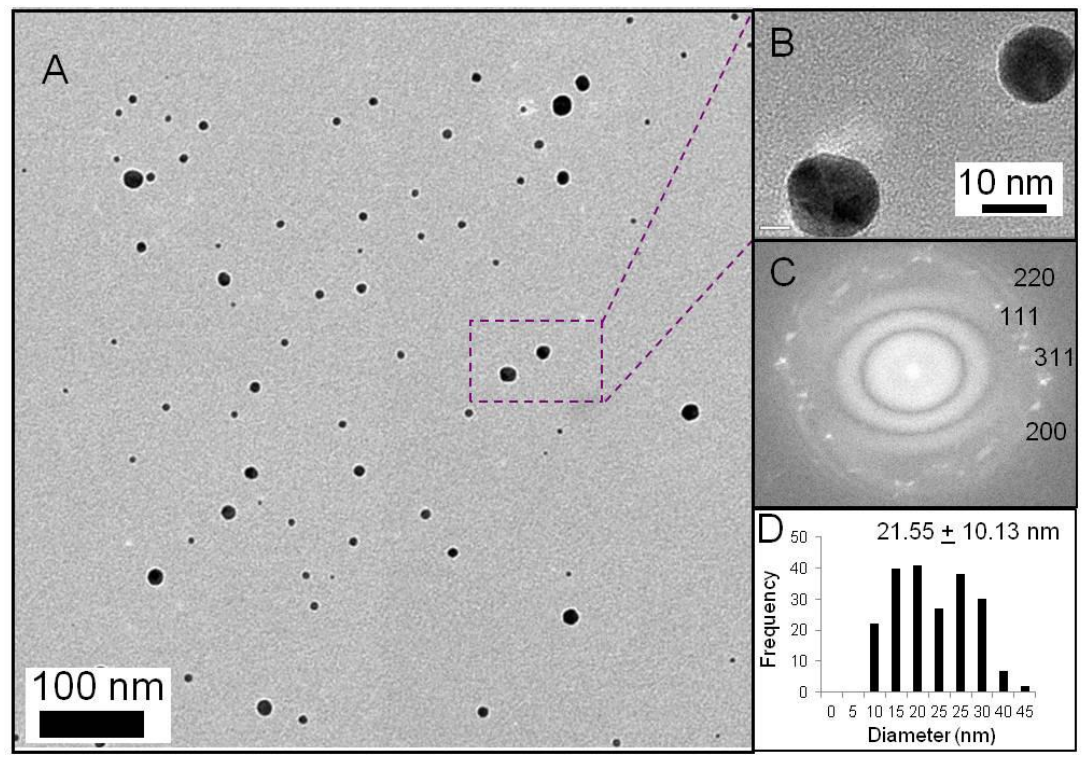

Figure 6.4. TEM images of the Au NPs resulting from the GA-Si NP $+\mathrm{HAuCl}_{4}$ photochemical reaction. (A., B.) C. FFT of the Au NPs image showing diffraction points consistent with $\mathrm{Au}^{0}$; D. Histogram of $200 \mathrm{Au}$ NPs illustrating the average particle diameter is $21.55 \pm 10.13 \mathrm{~nm}$.

The EDX spectrum of an isolated NP detected the characteristic X-rays consistent with the presence of $\mathrm{Cu}, \mathrm{C}, \mathrm{O}, \mathrm{Au}$, and $\mathrm{Si}$ (Figure 6.5). While $\mathrm{Cu}, \mathrm{C}$, and $\mathrm{O}$ are expected from the TEM grid and trace atmosphere, $\mathrm{C}$ and $\mathrm{O}$ may also be due to the presence of GA molecules on the Si NPs. Most notably, there were multiple peaks consistent with the characteristic X-rays of Au, further supporting the FFT diffraction pattern. While the characteristic X-rays from Si were detected, the signals were very low in intensity, as compared to $\mathrm{Au}$, consistent with the observation of few Si NPs while imaging. 


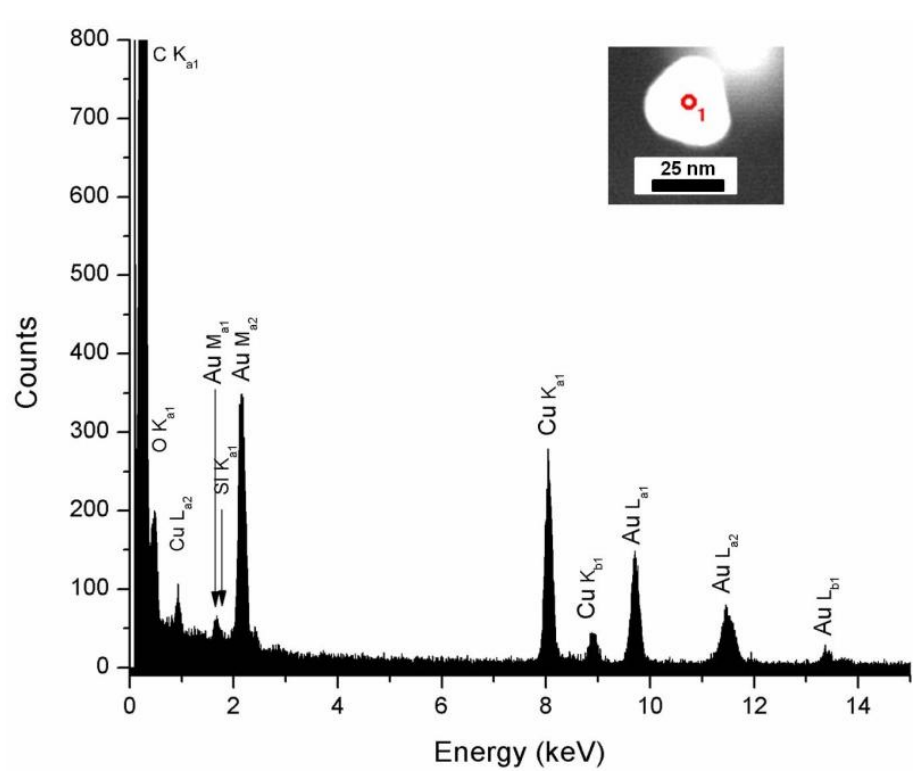

Figure 6.5. EDX spectrum of the NPs in the GA-Si NP $+\mathrm{HAuCl}_{4}$ photochemical reaction. Characteristic X-rays consistent with the presence of $\mathrm{Cu}, \mathrm{C}, \mathrm{O}, \mathrm{Au}$, and $\mathrm{Si}$ were detected.

XRD measurements of the GA-Si NP $+\mathrm{HAuCl}_{4}$ solution also indicated the formation of $\mathrm{Au}$ metal. The XRD pattern showed peaks correspond to the (111) and (200) planes of $\mathrm{Au}^{0}$ (Figure 6.6). When compared to the calculated $\mathrm{Si}^{0}$ pattern, no diffraction peaks from crystalline $\mathrm{Si}$ were detected. The lack of Si peaks in the XRD pattern could be due to: (1) the low concentration of $\mathrm{Si}$ NPs added to the $\mathrm{HAuCl}_{4}$ solution, (2) the relatively small size and weak scattering component of the Si NP as compared to the Au NPs, or (3) the oxidation of $\mathrm{Si}^{0}$ to amorphous $\mathrm{SiO}_{2}$ in the presence of $\mathrm{HAuCl}_{4}$. 


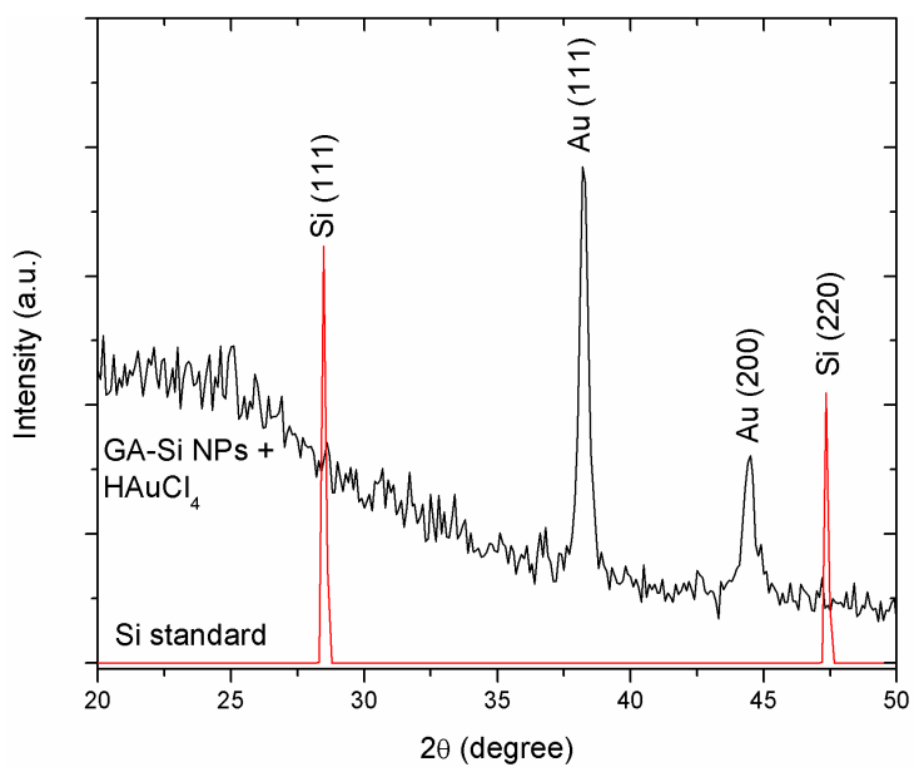

Figure 6.6. XRD pattern of the products of the GA-Si NP $+\mathrm{HAuCl}_{4}$ photochemical reaction. The obtained XRD pattern from the products of the GA-Si NPs $+\mathrm{HAuCl}_{4}$ photochemical reaction solution shows reflections consistent with the (111) and (200) planes of $\mathrm{Au}^{0}$. When compared to the $\mathrm{Si}^{0}$ standard, no $\mathrm{Si}^{0}$ peaks are observed.

Because the reduction of $\mathrm{AuCl}_{4}{ }^{-}$from $\mathrm{Au}^{3+}$ to $\mathrm{Au}^{0}$ and the formation of $\mathrm{Au}$ NPs is thought to be accomplished by the photo-oxidation of the GA-Si NPs, a series of control experiments (Table 6.1) were also performed. It was found that varying the GA-Si NPs concentration had little effect on the rate of the SPR growth (data not shown). However, when the $\mathrm{AuCl}_{4}{ }^{-}$concentration was increased, the SPR band was found to appear faster (data not shown). Neat $\mathrm{HAuCl}_{4}(0.2 \mathrm{~mL}, 0.15-10 \mathrm{mM})$ in $0.8 \mathrm{~mL}$ of $\mathrm{nH}_{2} \mathrm{O}$ was also exposed to UV light without the GA-Si NPs. Over 320 mins of UV light exposure, no visible color change or SPR band was observed (data not shown). This indicates that UV light does not initiate the formation of $\mathrm{Au} \mathrm{NPs}$ from $\mathrm{AuCl}_{4}{ }^{-}$in water. 
Table 6.1. GA-Si NP $+\mathrm{HAuCl}_{4}{ }^{-}$photochemical reactions and the effects on the SPR band formation.

\begin{tabular}{|c|c|}
\hline Experiment & SPR observation \\
\hline $\mathrm{AuCl}_{4}{ }^{-}+\mathrm{GA}-\mathrm{Si} \mathrm{NPs}+\mathrm{UV}$ light & Formed in $30 \mathrm{mins}$ \\
\hline Varying GA-Si NP concentration & No effect on SPR rate \\
\hline Increasing $\mathrm{AuCl}_{4}^{-}$concentration & Increased Rate of SPR Formation \\
\hline AuCl4- + UV light (no GA-Si NPs) & No SPR Observed \\
\hline $\mathrm{AuCl}_{4}^{-}+\mathrm{GA}-\mathrm{Si} \mathrm{NPs}$ (no UV light) & Formed in $4 \mathrm{hrs}$ \\
\hline $\mathrm{AuCl}_{4}^{-}+$neat GA + UV light (no Si NPs) & Formed in $3 \mathrm{hrs}$ \\
\hline
\end{tabular}

To determine if the reaction between $\mathrm{AuCl}_{4}{ }^{-}$and the GA-Si NPs was spontaneous (or required UV-irradiation), the UV absorption spectrum of a solution of $10 \mathrm{mM}$ $\mathrm{HAuCl}_{4}$ and GA-Si NPs $(0.2$ and $0.8 \mathrm{~mL}$, respectively) was also monitored under ambient conditions (Figure 6.7). No evidence of an SPR band was observed (line a) until the solution was aged for $4 \mathrm{hrs}$, when a very small SPR band at $615 \mathrm{~nm}$ was observed (line b). Though contrary to the expectation of size effects on the SPR with growth of the NP size over time, after $5 \mathrm{hrs,} \mathrm{this} \mathrm{peak} \mathrm{slightly} \mathrm{blue} \mathrm{shifts} \mathrm{to} 580 \mathrm{~nm}$ (line c). This peak is consistent with the Au SPR (Au NPs were confirmed in TEM, See Appendix), and suggest that the reaction between GA-Si NPs and $\mathrm{AuCl}_{4}{ }^{-}$appears spontaneous, but slow. The emission intensity of the GA-Si NPs also decreases in the presence of $\mathrm{HAuCl}_{4}{ }^{-}$under ambient conditions (Figure 6.7B). After 5 hrs, the GA-Si NP emission maximum was found to be $60 \%$ of the original intensity. To explain the decrease in the GA-Si NP emission intensity, it is reasonable that the photoexcited 
electrons in the GA-Si NPs are able to interact with, and reduce $\mathrm{AuCl}_{4}{ }^{-}$, rather than radiatively recombine with VB holes. Because reduction by GA-Si NPs is competitive with radiative recombination, reduction of $\mathrm{AuCl}_{4}{ }^{-}$to $\mathrm{Au}^{0}$ by the Si NPs should result in fewer recombination events, and thus less emission intensity.
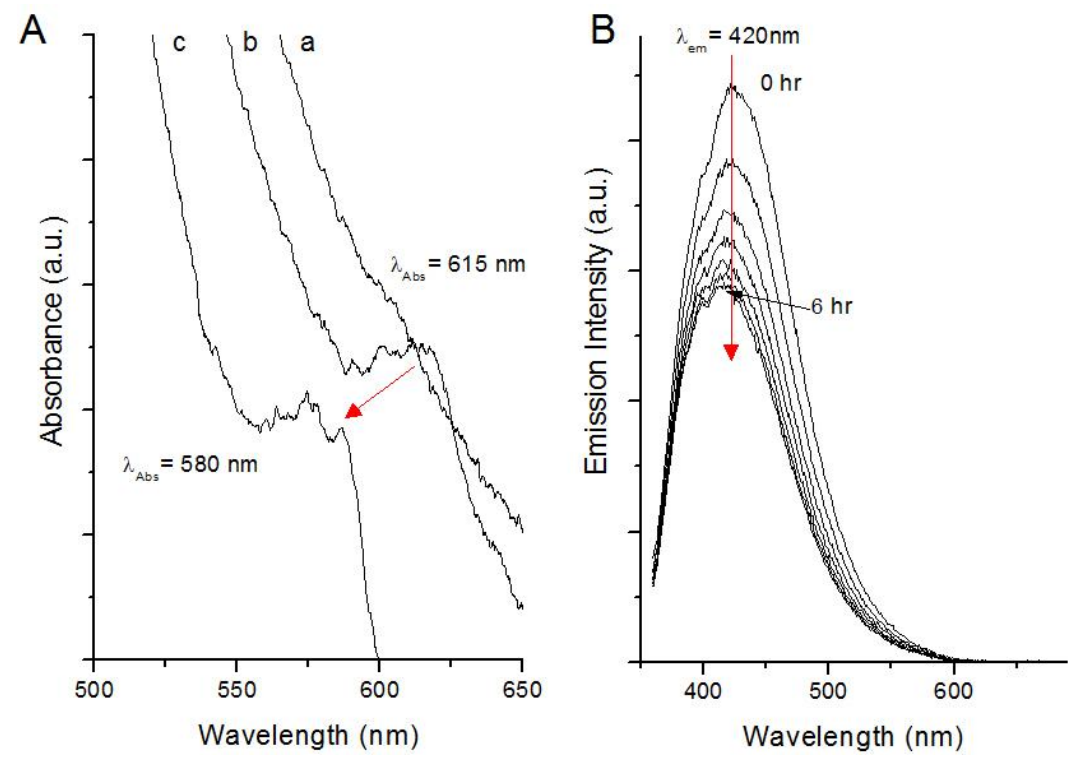

Figure 6.7. Photophysical properties of the GA-Si NP + $\mathrm{HAuCl}_{4}$ solution not exposed to UV light. A. UV-Vis spectra of $10 \mathrm{mM} \mathrm{HAuCl}_{4}+\mathrm{GA}-\mathrm{Si}$ NPs collected at $\mathrm{t}=0 \mathrm{hr}$ (line a), $\mathrm{t}=4 \mathrm{hr}$ (line $\mathrm{b}$ ), and $\mathrm{t}=5$ hours (line c) show an apparent SPR band, which blue shifts over time; B. The decreasing emission maximum ( $\left.420 \mathrm{~nm}, \lambda_{\mathrm{ex}}=350 \mathrm{~nm}\right)$ of the GA-Si NPs $+\mathrm{HAuCl}_{4}$ solution over time $(0-6 \mathrm{hrs})$.

The photo-reduction was also carried out with $10 \mathrm{mM} \mathrm{AuCl}_{4}{ }^{-}$in the presence of neat 1 M GA, likely an excess concentration as compared to the GA present in a GAsynthesized Si NP solution. After $3 \mathrm{hrs}$ of UV irradiation, a small peak at $305 \mathrm{~nm}$ was observed, consistent with the formation of Au NPs, which were confirmed using TEM (See Appendix). However, because the SPR was obtained after 3 hrs of UV irradiation, this reaction is slower than the photochemical reaction in the presence of the GA-Si NPs, 
suggesting the Si NPs are necessary for efficient formation of Au NPs in this photochemical system.

\subsubsection{Photochemical Reaction of the GA-Si NPs with $\mathrm{Ag}^{+}$}

$\mathrm{AgNO}_{3}$ was added to the GA-Si NPs, in place of $\mathrm{HAuCl}_{4}$, to determine if $\mathrm{Ag}^{+}$ could also be formed photochemically. The absorption spectrum of neat $\mathrm{AgNO}_{3}$ is shown in Figure 6.8A (line a) which shows an absorption peak at $260 \mathrm{~nm}$ (See Appendix). The absorbance spectra of $\mathrm{AgNO}_{3}$ in the presence of the GA-Si NPs are also shown in Figure 6.8A (lines b-d). After only 2 mins of UV irradiation, a new absorbance peak at $395 \mathrm{~nm}$ was observed (line b), which an increase in intensity up to $10 \mathrm{mins}$ of irradiation (line d). After 10 mins, the intensity of the absorption peak at $395 \mathrm{~nm}$ was observed to decrease, with a shoulder emerging at $510 \mathrm{~nm}$ becoming more obvious. During this time, the color of the GA-Si NP $+\mathrm{AgNO}_{3}$ solution changed from colorless to dark purple (see inset). Similar to the addition of $\mathrm{HAuCl}_{4}$ and the observation of the $\mathrm{Au}$ NP SPR band, the broad, visible absorption with peak intensity at 395 and $510 \mathrm{~nm}$ is

consistent with the SPR band ${ }^{[142 \mathrm{~b}, 142 \mathrm{c}]}$ of $\mathrm{Ag}^{0}$. With increasing UV irradiation (up to 90 min., Figure $6.8 \mathrm{~B}$, line $\mathrm{g}$ ), the absorption peaks at 395 and $510 \mathrm{~nm}$ continued to decrease to the baseline, while continuing to red shift. The SPR red shift and decreased intensity are potentially due to formation of increasingly larger Ag NPs with time, which are larger than the expected size for Ag which exhibit SPR. 

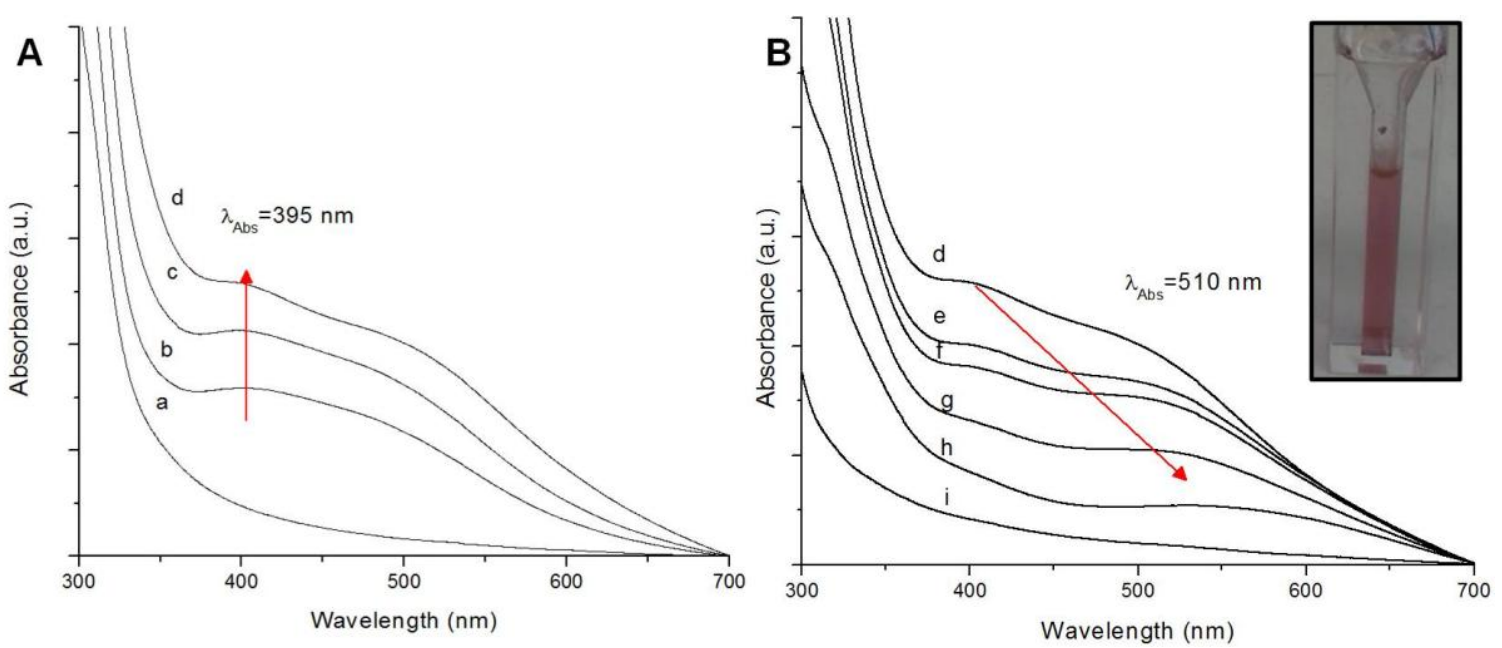

Figure 6.8. Absorption spectra of neat $\mathrm{AgNO}_{3}$ and the GA-Si $\mathrm{NP}+\mathrm{AgNO}_{3}$ photochemical reaction. A.

Line a shows the absorption spectrum of neat $\mathrm{AgNO}_{3}$. The GA-Si NPs and $\mathrm{AgNO}_{3}$ were irradiated with UV light for various times resulting in the absorbance growth at $395 \mathrm{~nm}$ : line $\mathrm{b}, \mathrm{t}=2 \mathrm{mins}$, line $\mathrm{c}, \mathrm{t}=5 \mathrm{mins}$, line $\mathrm{d}, \mathrm{t}=10 \mathrm{mins}$; $\mathrm{B}$. The absorbance peak at $395 \mathrm{~nm}$ decreases with increased UV irradiation time, while a shoulder at $510 \mathrm{~nm}$ becomes more distinct. This peak also decreases under continuous UV irradiation: line $\mathrm{d}, \mathrm{t}=10$ mins, line $\mathrm{e}, \mathrm{t}=20$ mins, line $\mathrm{f}, \mathrm{t}=30 \mathrm{~min}$, line $\mathrm{g}, \mathrm{t}=60$ mins, line $\mathrm{h}, \mathrm{t}=90 \mathrm{mins}$, line $\mathrm{i}, \mathrm{t}=300 \mathrm{mins}$.

The emission intensity of the GA-Si NPs was also affected by the presence of $\mathrm{Ag}^{+}$. While the emission maximum at $420 \mathrm{~nm}\left(\lambda_{\mathrm{ex}}=350 \mathrm{~nm}\right)$ was observed to decrease over time (Figure 6.9), this emission quenching was slower and more gradually than after the addition of $\mathrm{HAuCl}_{4}$; with $\mathrm{Ag}^{+}$after 60 and 300 mins, the emission maximum of the GA-Si NPs was $34 \%$ and $15 \%$ of the original emission intensity, respectively, as compared to the addition of $\mathrm{AuCl}_{4}{ }^{-}$at similar time points. 


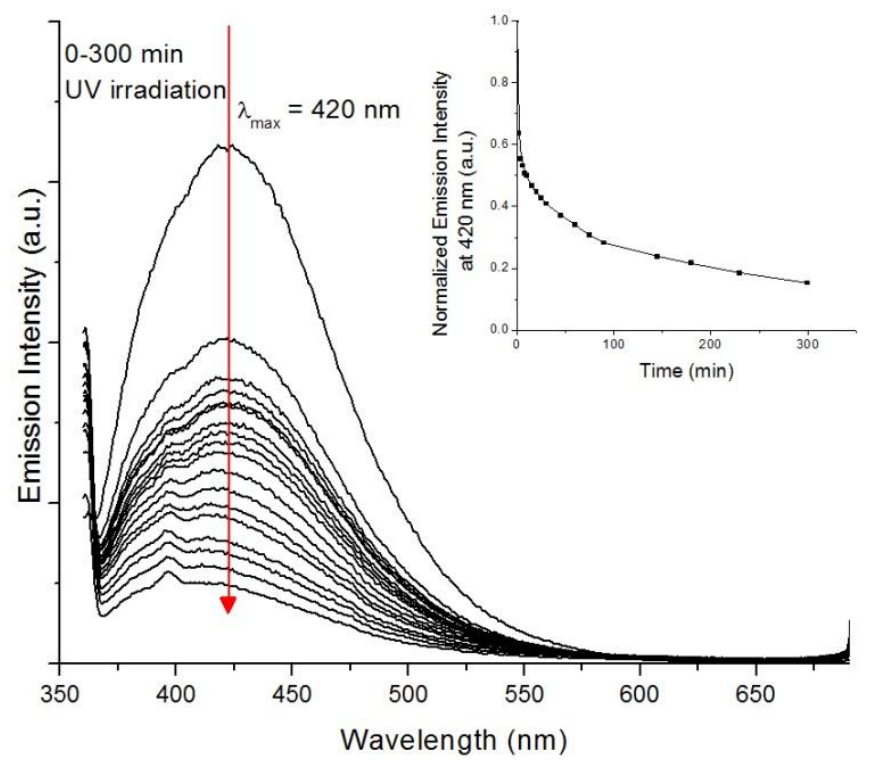

Figure 6.9. Emission spectra of time for the GA-Si NP $+\mathrm{AgNO}_{3}$ photochemical reaction. The emission maximum of the GA-Si NPs $\left(420 \mathrm{~nm}, \lambda_{\mathrm{ex}}=350 \mathrm{~nm}\right)$ in the presence of $10 \mathrm{mM} \mathrm{AgNO}{ }_{3}$ decreases with increasing UV irradiation time, up to 300 mins. Inset : The normalized emission intensity of the GA-Si NPs at $420 \mathrm{~nm}$ versus UV irradiation time.

The GA-Si NP emission quench in the presence of $\mathrm{AgNO}_{3}$ and UV irradiation, in addition to the new absorption, characteristic of the Ag SPR, indicated that Ag NPs were forming by the photochemical reduction of $\mathrm{Ag}^{+}$by the Si NPs. A TEM image of the GA$\mathrm{Si} \mathrm{NP}+\mathrm{AgNO}_{3}$ photochemical reaction after 10 mins of UV irradiation shows the presence of many spherical particles (Figure 6.10A), while the HR-TEM image shows that these particles are crystalline (Figure 6.10B). Many diffraction planes consistent with $\mathrm{Ag}^{0}$ were observed by SAED, including the (111) at $2.40 \AA$, (200) at $2.00 \AA$, (220) at $1.45 \AA,(311)$ at $1.23 \AA$, and (400) at $1.18 \AA$, consistent with JCPDS card no. 001-1164 (Figure 6.10C). 
The red shift in the UV-Vis spectra of the Ag NP SPR band suggests that with increased UV irradiation time, larger Ag NPs are formed. To examine this, TEM images of particles obtained after 10 mins of UV irradiation were compared to the TEM images of particles after 60 mins of UV irradiation. The diameter of the Ag NPs obtained from the $\mathrm{GA}-\mathrm{Si} \mathrm{NP}+\mathrm{AgNO}_{3}$ photochemical reaction after 10 mins was determined to be $11.77 \pm 3.14 \mathrm{~nm}$. The average NP diameter after 60 mins of UV-irradiation was found to be slightly larger, at $12.91 \pm 4.65 \mathrm{~nm}$. This increase in size as observed by TEM is consistent with the red shift in the SPR band observed in the UV-Vis with increased UV irradiation time. EDX on both samples found $\mathrm{Ag}, \mathrm{Cu}$ (grid), $\mathrm{O}$, and $\mathrm{C}$, supporting the presence of Ag NPs (data not shown). Characteristic X-rays consistent with the presence of Si were also detected, but were very low in intensity.

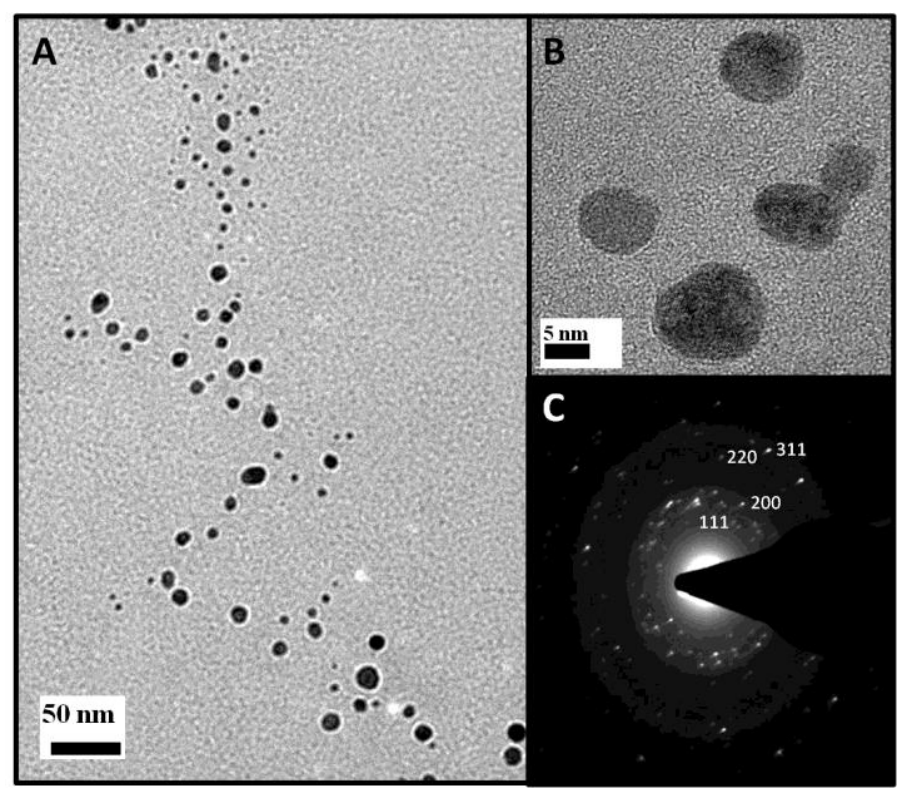

Figure 6.10. TEM images of the Ag NPs resulting from the GA-Si NPs $+\mathrm{AgNO}_{3}$ photochemical reaction $(\mathrm{A}, \mathrm{B})$. TEM images of $\mathrm{Ag} \mathrm{NPs}$ resulting from the $\mathrm{GA}-\mathrm{Si} \mathrm{NPs}+\mathrm{AgNO}_{3}$ solution after 10 mins of UV irradiation. C. SAED pattern of Ag NPs showing the diffraction reflections corresponding to the (111), (200), (220), and (311) planes of $\mathrm{Ag}^{0}$. 
Control experiments involving neat $\mathrm{AgNO}_{3}(0.2 \mathrm{~mL}, 0.1-10 \mathrm{mM})$ in $\mathrm{nH}_{2} \mathrm{O}(0.8$ $\mathrm{mL}$ ) were performed by exposed it to UV irradiation. Similar to the Au control, no SPR band formation or color change was observed over 60 min, which supported that the GASi NPs are necessary to efficiently generate noble metal NPs in this photochemical system. Additionally, without UV irradiation, the $\mathrm{GA}-\mathrm{Si} \mathrm{NP}+\mathrm{AgNO}_{3}$ solution produced an SPR band at $515 \mathrm{~nm}$ after 3 hours (Figure 6.11A). Additionally, the GA-Si NP emission was found to decrease over time, supporting that the reaction between the $\mathrm{AgNO}_{3}$ and the GA-Si NPs (Figure 6.11B).
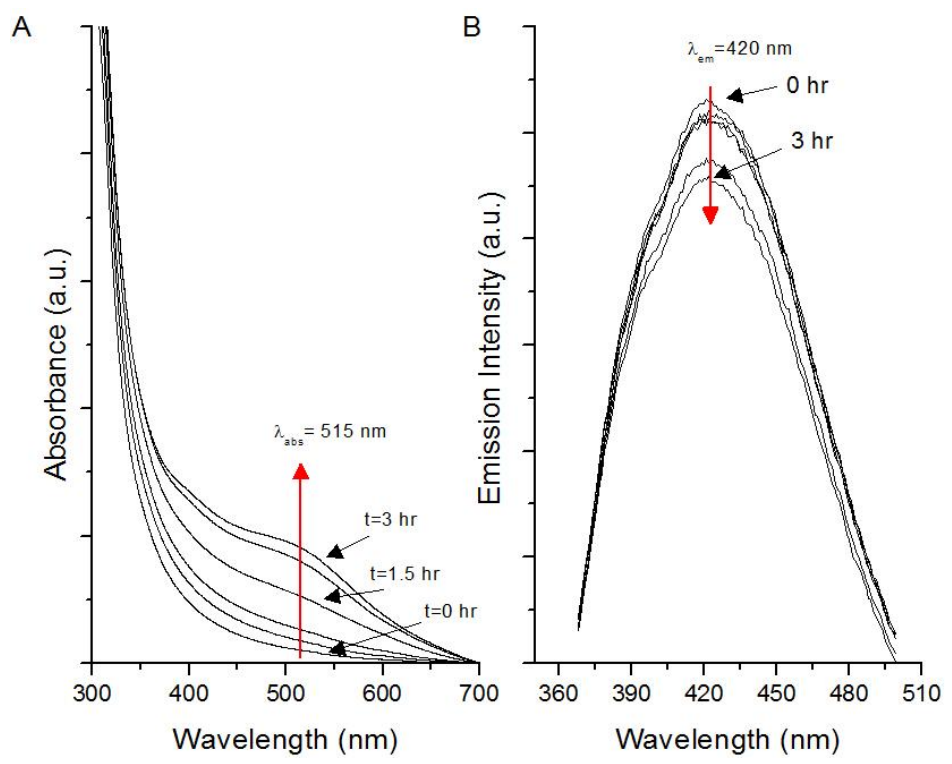

Figure 6.11. Absorbance and emission spectra of the GA-Si NPs $+\mathrm{AgNO}_{3}$ not exposed to UV light. A. UV spectra of $10 \mathrm{mM} \mathrm{AgNO}_{3}$ and GA-Si NPs collected over 3 hrs with an apparent SPR band observed at $515 \mathrm{~nm}$. B. The decreasing emission spectra $\left(\lambda_{\mathrm{ex}}=350 \mathrm{~nm}\right)$ of the GA-Si NPs and $\mathrm{AgNO}_{3}$ over 3 hrs. 
6.4. Photochemical Reactions of the red-emitting the $\mathrm{SiO}_{\mathrm{x}}-\mathrm{Si}$ NPs with Various Metal Ions

\subsubsection{Characterization of $\mathrm{SiO}_{\mathrm{x}}-\mathrm{Si} \mathrm{NPs}$}

The $\mathrm{SiO}_{\mathrm{x}}-\mathrm{Si}$ NPs used in this study were prepared by the controlled oxidation of hydride-terminated $\mathrm{Si}$ NPs in the presence of ethanol and $\mathrm{H}_{2} \mathrm{O}_{2} \cdot{ }^{[141]}$ The obtained $\mathrm{Si} / \mathrm{SiH}_{\mathrm{y}} \mathrm{O}_{\mathrm{x}}$ core/shell NPs were directly dispersible in water and were characterized using TEM and SAED. The $\mathrm{SiO}_{\mathrm{x}}-\mathrm{Si} \mathrm{NPs}$ were found to have crystalline $\mathrm{Si}$ cores (ca. $6 \mathrm{~nm}$ ) embedded in a $\mathrm{SiO}_{2}$ matrix. As observed by FT-IR, the $\mathrm{SiO}_{x}-\mathrm{Si}$ NPs exhibited both $\mathrm{Si}-\mathrm{O}$ and $\mathrm{Si}-\mathrm{H}$ stretches, consistent with mixed hydride and oxide surface termination (data not shown).

\subsubsection{Photostability of the neat $\mathrm{SiO}_{\mathrm{x}}-\mathrm{Si}$ NPs}

The absorption and emission spectra of the $\mathrm{SiO}_{\mathrm{x}}-\mathrm{Si}$ NPs are shown in Figure 6.12. The absorption spectrum of the $\mathrm{SiO}_{\mathrm{x}}-\mathrm{Si}$ NPs (Figure 6.12A, line a) shows an absorption edge at $450 \mathrm{~nm}$ and continuous absorption at shorter wavelengths into the UV, with but no obvious shoulders. The $\mathrm{SiO}_{\mathrm{x}}-\mathrm{Si}$ NPs were observed to have an emission maximum at $579 \mathrm{~nm}$ when excited at $350 \mathrm{~nm}$ (Figure 6.12B), and were found to be stable under ambient conditions for several weeks (data not shown). The stock solution of the $\mathrm{SiO}_{\mathrm{x}}-\mathrm{Si} \mathrm{NPs}$, however, is not as bright as the GA-Si NP emission, potentially due to a lower Si concentration.

\subsubsection{Photochemical Reaction of the $\mathrm{SiO}_{\mathrm{x}}-\mathrm{Si}$ NPs with $\mathrm{AuCl}_{4}^{-}$}

When $\mathrm{HAuCl}_{4}$ was added to the $\mathrm{SiO}_{\mathrm{x}}-\mathrm{Si} \mathrm{NP}$ solution (Figure 6.12), an immediate color change was observed visually (Figure 6.12, inset) and a peak at $560 \mathrm{~nm}$ was 
observed in the absorbance spectrum. After formation, the absorption intensity at $560 \mathrm{~nm}$ decreased with increased UV irradiation time. The $\mathrm{SiO}_{\mathrm{x}}-\mathrm{Si} \mathrm{NP}$ emission maximum at $579 \mathrm{~nm}$ also decreased within 2 mins of $\mathrm{UV}$ irradiation in the presence of $\mathrm{HAuCl}_{4}$ (Figure 6.12B). However, due to the weak initial emission of the $\mathrm{SiO}_{\mathrm{x}}-\mathrm{Si} \mathrm{NPs}$, it is difficult to ascertain the extent to which the quenching is occurring after 2 mins.
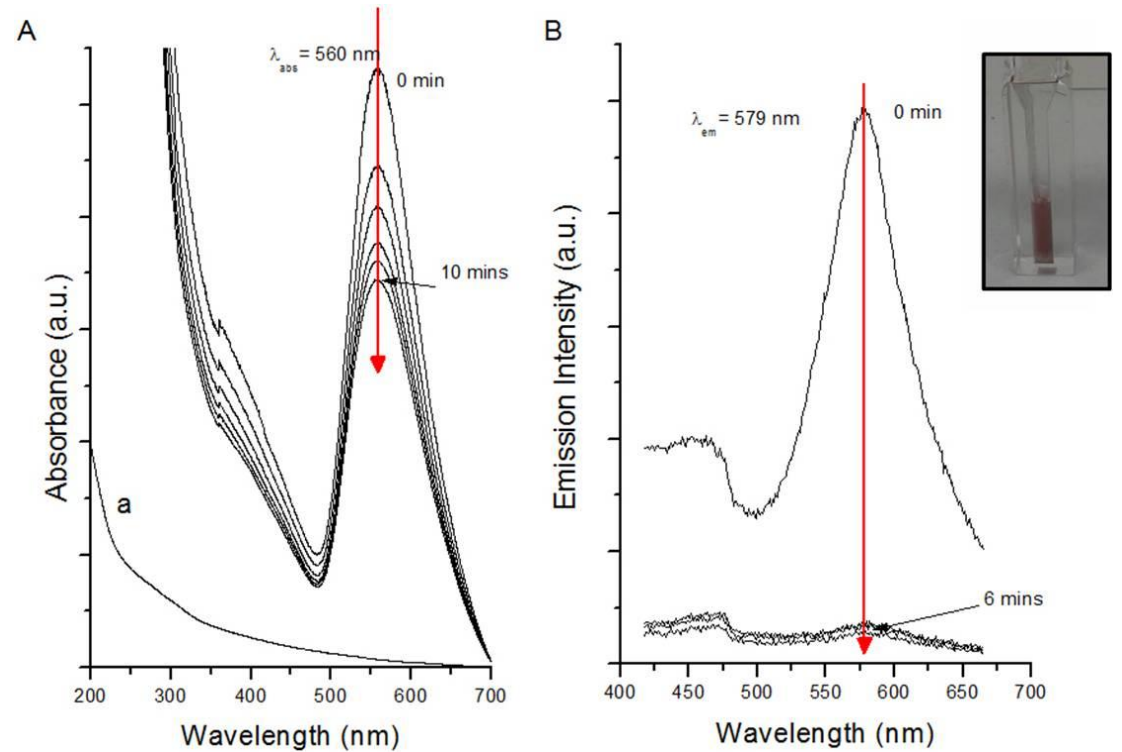

Figure 6.12. Absorbance and emission spectra of the $\mathrm{SiO}_{\mathbf{x}}-\mathrm{Si} \mathrm{NP}+\mathrm{HAuCl}_{4}$ photochemical reaction. A. Absorbance spectra of the neat $\mathrm{SiO}_{\mathrm{x}}-\mathrm{Si} \mathrm{NPs}$ (line a) and $\mathrm{SiO}_{\mathrm{x}}-\mathrm{Si} \mathrm{NPs}+\mathrm{HAuCl}_{4} \mathrm{UV}$ irradiated for various times $(0-10 \mathrm{mins})$. Immediately upon addition, an absorption peak at $560 \mathrm{~nm}$ is observed; B. The emission maxima of the $\mathrm{SiO}_{\mathrm{x}}-\mathrm{Si} \mathrm{NPs}(579 \mathrm{~nm})+\mathrm{HAuCl}_{4}$ decreases with increasing UV irradiation times (0-6 mins); Inset. Digital image of the $\mathrm{SiO}_{\mathrm{x}}-\mathrm{Si} \mathrm{NP}+\mathrm{HAuCl}_{4}$ solution immediately after mixing.

While many factors affect SPR band position, the red shifted SPR compared to that observed from the GA-Si NPs $+\mathrm{HAuCl}_{4}$ solutions suggests the Au NPs from the $\mathrm{SiO}_{\mathrm{x}}-\mathrm{Si} \mathrm{NP}$ photochemical reaction would be larger than the $\mathrm{Au}$ NPs from the $\mathrm{HAuCl}_{4}$ and GA-Si NP solution. TEM images of the products obtained from the photochemical reaction of the $\mathrm{HAuCl}_{4}{ }^{-}$and $\mathrm{SiO}_{\mathrm{x}}-\mathrm{Si} \mathrm{NPs}$ are shown in Figure 6.13. The obtained $\mathrm{Au}$ 
NPs are spherical, highly crystalline, and faceted, with an average size of $33.48 \pm 13.54$ $\mathrm{nm}$, supporting the red-shifted absorption spectra.

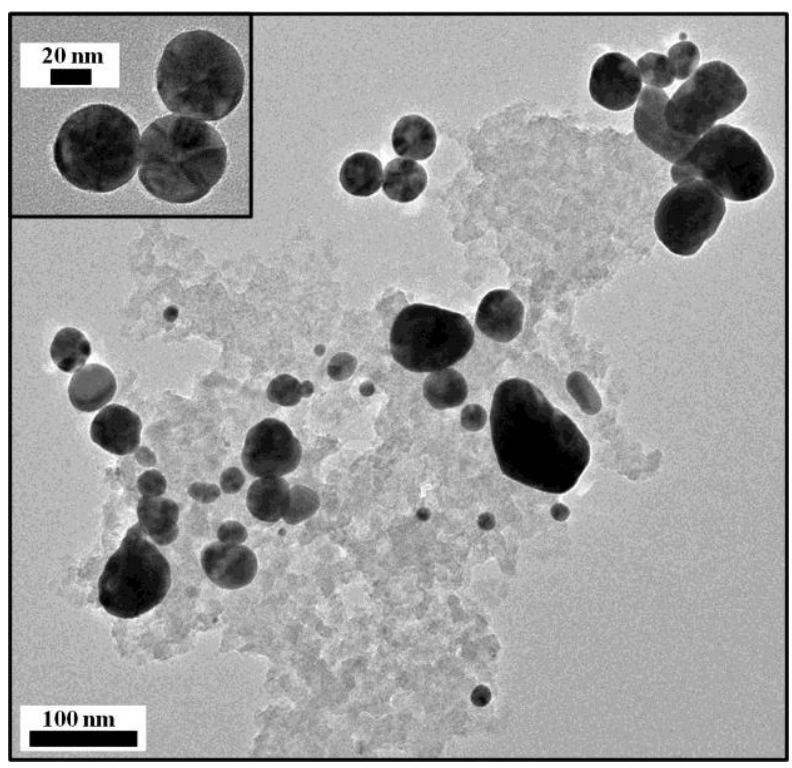

Figure 6.13. TEM images of Au NPs resulting from $\mathrm{SiO}_{\mathrm{x}}-\mathrm{Si} \mathrm{NP}+\mathrm{HAuCl}_{4}$ photochemical reaction.

\subsubsection{Photostability of the $\mathrm{SiO}_{\mathrm{x}}-\mathrm{Si}$ NPs in the presence of $\mathrm{Ag}^{+}$}

$\mathrm{AgNO}_{3}$ was also added to the $\mathrm{SiO}_{\mathrm{x}}-\mathrm{Si} \mathrm{NP}$ solution to determine if $\mathrm{Ag}$ NPs could be formed in the presence of intense UV light. After 120 mins of UV-irradiation, no SPR band or color change was ever observed from the $\mathrm{SiO}_{\mathrm{x}}-\mathrm{Si}$ NPs and $\mathrm{AgNO}_{3}$ solution (Figure 6.14). 


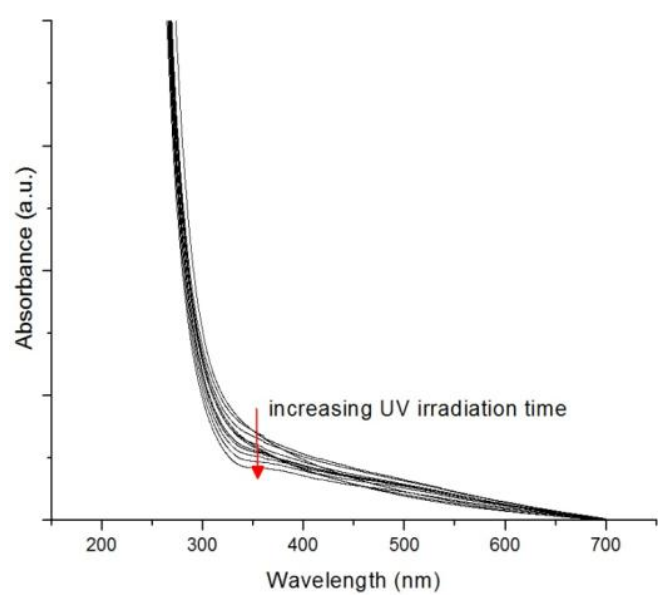

Figure 6.14. Absorption spectra of the $\mathrm{SiO}_{\mathbf{x}}-\mathbf{S i} \mathrm{NPs}_{\mathbf{A g N O}} \mathbf{A g}_{3}$. Absorption spectra of the $\mathrm{SiO} \mathrm{x}_{\mathrm{x}}-\mathrm{Si} \mathrm{NPs}^{+}$ $\mathrm{AgNO}_{3}$ at various UV irradiation times; a SPR band was not observed.

\subsection{Discussion}

Whether or not a redox reaction occurs is dependent upon the reduction potentials of the species in the system. The standard reduction potential of $\mathrm{AuCl}_{4}{ }^{-}$is given by the following reaction,
$\mathrm{AuCl}_{4}^{-}+3 \mathrm{e}^{-} \rightarrow \mathrm{Au}^{0}+4 \mathrm{Cl}^{-}$
$(+0.93 \mathrm{~V})$

while the reduction potential of bulk $\mathrm{Si}^{0}$ is given by,

$\mathrm{Si}^{4+}+4 \mathrm{e}-\rightarrow \mathrm{Si}^{0}$

When $\mathrm{AuCl}_{4}{ }^{-}$and $\mathrm{Si}^{0}$ are present together, the reduction reaction is spontaneous with a potential of $1.77 \mathrm{~V}$, according to,

$4 \mathrm{AuCl}_{4}^{-}+3 \mathrm{Si}^{0} \rightarrow 4 \mathrm{Au}^{0}+3 \mathrm{Si}^{4+}+4 \mathrm{Cl}^{-}$

Therefore, when $\mathrm{AuCl}_{4}{ }^{-}$is added to the GA-Si NPs, the $\mathrm{Au}^{3+}$ ions can react with the crystalline Si atoms to spontaneously produce Au NPs. As it turns out, in order for the reduction of $\mathrm{AuCl}_{4}{ }^{-}$by the GA-Si NPs to be efficient, the solution needs to be exposed to 
UV light. Even under UV irradiation, this reaction takes up over 30 mins before a significant SPR band is observed in the UV-Vis. The delayed observation of the Au SPR band may be due to the presence of the oxidized Si NP surface. The blue emission of the GA-Si NPs, which may originate from 1-2 nm Si nanocrystals that are embedded in a silicon suboxide matrix, exhibits an emission dependence on the excitation energy; this dependence, corresponding NP size, and emission color may be consistent with emission originating from QC. Due to the surface oxidation, excitons, particularly electrons, tunneling from the Si core to the surface may not be particularly fast (or efficient), and therefore a sufficient number of reduction reactions to produce the SPR band from Au NP may take time to occur. If electrons are tunneling from the core to the surface to reduce $\mathrm{AuCl}_{4}{ }_{4}$, then this may also explain why the emission intensity of the GA-Si NPs in the presence of $\mathrm{AuCl}_{4}{ }^{-}$decreases over time: electrons that reduce $\mathrm{AuCl}_{4}{ }^{-}$are no longer available for radiative recombination across the bandgap, which results in lower Si NP emission intensity.

Based on the presence of $\mathrm{Si}^{0}$, the addition of $\mathrm{AuCl}_{4}{ }^{-}$to the $\mathrm{SiO}_{\mathrm{x}}-\mathrm{Si} \mathrm{NP}$ solution is also expected to spontaneously produce Au NPs. Tthe red-emission from the $\mathrm{SiO}_{\mathrm{x}}-\mathrm{Si}$ NP solution, however, does not possess an emission dependence on excitation energy. It is possible that the red emission event could originate from the recombination of excitons formed within the $\mathrm{Si}^{0}$ nanocrystalline core at defect sites on the Si NP surface. With emission events originating from the $\mathrm{Si}$ surface, the electrons from the $\mathrm{SiO}_{\mathrm{x}}-\mathrm{Si} \mathrm{NPs}$ may already tunnel to the NP surface. If this were the origin of the red emission event, then this may help explain why the addition of $\mathrm{AuCl}_{4}{ }^{-}$to the $\mathrm{SiO}_{\mathrm{x}}-\mathrm{Si}$ NPs results in an 
immediate color change of the $\mathrm{SiO}_{\mathrm{x}}-\mathrm{Si} \mathrm{NP}+\mathrm{AuCl}_{4}{ }^{-}$solution, the immediate formation of the Au SPR band, and the immediate decrease in the $\mathrm{SiO}_{\mathrm{x}}-\mathrm{Si} \mathrm{NP}$ emission.

However, the reaction of bulk $\mathrm{Si}^{0}$ and $\mathrm{Ag}^{+}$is different from the expected reaction of $\mathrm{Si}^{0}$ with $\mathrm{AuCl}_{4}^{-}$. The standard reduction potential of $\mathrm{Ag}^{+}$is given by,
$\mathrm{Ag}^{+}+\mathrm{e}^{-} \rightarrow \mathrm{Ag}^{0}$
$(+0.7996 \mathrm{~V})$
(Eq. 6.4)

In the presence of bulk $\mathrm{Si}^{0}$, the reduction of $\mathrm{Ag}^{+}$is not predicted to be spontaneous because the overall reaction potential is negative $(\sim-0.04 \mathrm{~V})$. Little work, however, has focused on determining the effects of NP size on the reduction potential; if Si NPs are capable of reducing $\mathrm{Ag}^{+}$, then QC-based size effects would be attributed for this change in Si NP reduction potential. Because the reduction of $\mathrm{Ag}^{+}$to $\mathrm{Ag}$ NPs spontaneously occurs in the presence of the GA-Si NPs and not the $\mathrm{SiO}_{\mathrm{x}}-\mathrm{Si} \mathrm{NPs}$, it further supports that the emission from the GA-Si NPs is influenced by QC while the $\mathrm{SiO}_{\mathrm{x}}-\mathrm{Si}$ NPs are less affected. The reduction potential of the GA-Si NPs may be decreased, in accordance with QC, such that it they are capable of reducing $\mathrm{Ag}^{+}$; this would require the potential to be greater than $-0.7996 \mathrm{~V}$ in order to spontaneously reduce $\mathrm{Ag}^{+}$. The reduction potential from species whose emission originates from surface defects sites, as may be the case of the $\mathrm{SiO}_{\mathrm{x}}-\mathrm{Si}$ NPs, likely have a reduction potential that is less affected by QC and that still resembles that of bulk $\mathrm{Si}^{0}(-0.84 \mathrm{~V})$. This difference in reduction potentials of Si NPs indicates that the origin of emission may be different, and could explain why the GA-Si NPs are capable of reducing $\mathrm{Ag}^{+}$to $\mathrm{Ag}^{0}$ to form $\mathrm{Ag}$ NPs, while the $\mathrm{SiO}_{\mathrm{x}}-\mathrm{NPs}$ are not.

Going forward, this study may help lend insight into, not only the origin of Si NP emission, but also the effects of QC on semiconductor reduction potentials. The 
reduction potential of Si NPs may be further investigated through the addition of other ions with well-characterized reduction potentials; if a metal is not reduced by the Si NPs, than likely the metal reduction potential is below that of the Si NPs. Ideally, a range of reduced and unreduced metals can help estimate the Si NP reduction potentials.

\subsection{Conclusion}

Blue-(glutaric acid-synthesized Si NPs, GA-Si NPs) and red $\left(\mathrm{SiO}_{\mathrm{x}} / \mathrm{H}_{\mathrm{y}}-\mathrm{Si} \mathrm{NPs}\right.$, $\mathrm{SiO}_{\mathrm{x}}-\mathrm{Si} \mathrm{NPs}$ ) emitting $\mathrm{Si}$ NPs were exposed to metal salts. This interaction affected, not only the emission spectra of the Si NPs, but also the absorption spectra of the metal ions. When $\mathrm{HAuCl}_{4}$ was added to the GA-Si NPs, a decrease in the Si NP emission occurred alongside the increase of the surface plasmon resonance band (SPR), consistent with the formation of $\mathrm{Au}$ NPs. $\mathrm{AgNO}_{3}$ was also added to the GA-Si NPs, and resulted in the formation of the $\mathrm{Ag} \mathrm{SPR}$ band and $\mathrm{Ag} \mathrm{NPs} . \mathrm{HAuCl}_{4}$ and $\mathrm{AgNO}_{3}$ were also added to redemitting $\mathrm{SiO}_{\mathrm{x}}-\mathrm{Si}$ NPs, NPs; the addition of $\mathrm{HAuCl}_{4}$ to the $\mathrm{SiO}_{\mathrm{x}}-\mathrm{Si}$ NPs caused an immediate color change and SPR band formation, consistent with the Au NPs. However, $\mathrm{Ag}$ NPs were not observed from the $\mathrm{SiO}_{\mathrm{x}}-\mathrm{Si} \mathrm{NP}+\mathrm{AgNO}_{3}$ photochemical reaction. Based on the reduction potentials, bulk $\mathrm{Si}^{0}$ is expected to reduce $\mathrm{AuCl}_{4}{ }^{-}$; therefore, the formation of $\mathrm{Au}$ NPs from the GA- and $\mathrm{SiO}_{\mathrm{x}}-\mathrm{Si}$ NPs solutions in the presence of $\mathrm{AuCl}_{4}{ }^{-}$

was expected. However, the reduction potential of bulk $\mathrm{Si}^{0}$ is much less than that of $\mathrm{Ag}^{+}$ and the reduction to Ag NPs was not expected. The GA-Si NPs, whose emission may originate from the quantum confinement (QC) effects on the Si bandgap, may also have a reduction potential that is also affected by $\mathrm{QC}$, and may explain why these particles can 
photo-reduce $\mathrm{Ag}^{+}$. Conversely, the $\mathrm{SiO}_{\mathrm{x}}-\mathrm{Si}$ NPs may have an emission that originates from the radiative recombination of excitons at surface defect sites, and a reduction potential that may not be affected by QC. This would explain why the $\mathrm{SiO}_{\mathrm{x}}-\mathrm{Si} \mathrm{NPs}$ cannot reduce $\mathrm{Ag}^{+}$. Because work on determining the reduction potential of NPs has not readily been addressed, this study suggests that the further addition of metal ions to $\mathrm{Si}$ NPs may allow the reduction potentials of the Si NPs to be determined, which can also give insight into the origin of Si NP emission. 


\title{
Synthesis and Emission Properties of Red-Emitting, Ester-Functionalized Silicon
}

\section{Nanoparticles}

\begin{abstract}
The origin of nanosized silicon $(\mathrm{Si})$ emission has been debated, as both particle size and surface-related defects have been found to cause unintended shifts in Si nanoparticle (NP) emission. In efforts to better to protect the observed emission of Si NPs, increased surface termination groups or molecular barriers have been used. Presented here is the thermal hydrosilylation of hydride-terminated Si NPs $(5.51+1.35 \mathrm{~nm})$ with bi-functional esters of different hydrocarbon chain lengths (5 and 11-carbon atoms) with methyl or tert-butyl ester terminations. It has been previously observed that short chain groups more efficiently passivate the Si NP surface, but it may be possible that the tert-butyl ester moiety will provide a better barrier to protect the Si NP surface from small molecule oxidants. To test this hypothesis, red-emitting $\left(\lambda_{\mathrm{em}}=575 \mathrm{~nm}\right)$ ester-functionalized Si NPs were dispersed in straight chain alcohols, including methanol, pentanol, and decanol. When the methoxy ester-functionalized Si NPs were exposed to the alcohols, the emission immediately changed to blue. However, the tert-butyl-4-pentenoatefunctionalized Si NPs retained a red emission when exposed to the alcohols. This suggests that the barrier method of protecting the Si NP surface may be more efficient than increased surface coverage.
\end{abstract}




\subsection{Introduction}

The discovery of efficient visible photoluminescence (PL) from porous silicon ${ }^{[18 \mathrm{a}]}$ has attracted great interest, due to its potential use in applications within silicon-based optoelectronic devices and as cellular labeling probes. ${ }^{[6 \mathrm{~d}, 6 f, 91,144]}$ Since then, work on freestanding $\mathrm{Si}$ nanoparticles (NPs) has focused on preparing monodisperse, functionalized Si NPs, while protecting their emission characteristics. This becomes more complicated because the origin of Si NP emission is not fully understood. The predominant theories ${ }^{[90 \mathrm{a}, 99]}$ concerning Si emission can be categorized into three types: 1) Quantum Confinement (QC), 2) Luminescent Centre (LC), and 3) Quantum Confinement-Luminescent Centre (QC/LC). QC-based, as observed in direct band-gap II-VI semiconductor quantum dots (QDs), originates from the radiative recombination following exciton annihilation within the semiconductor NP core. Because the size of the nanocrystal effects the bandgap, QC-based emission is tunable by Si NP size. ${ }^{[69]}$ The LC model involves self-trapped excitons with direct excitation and emission, which gives rise to visible $\mathrm{PL}^{[90 \mathrm{~b}]}$ and has been generally attributed to various oxide moieties formed on unoccupied Si surfaces. ${ }^{[145]}$ QC/LC is a hybrid model, and involves exciton generation within the NP core which can decay at surface defect sites, resulting in emissive events. $^{[146]}$ However, because competing emission events can be present, often manifesting as both simultaneous blue and red emission, ${ }^{[4,22]}$ it is difficult to separate the size-dependent core from the size-independent surface effects.

Regardless of the origin of Si NP emission, functional surface groups are used to terminate the NPs with substituents that are both useful, while preserving the observed 
optical properties. This dual purpose of surface groups has become especially important for red-to-NIR-emitting Si NPs, which are potential biological fluorophores; fluorophores in this emission range are strongly preferred, as emission from biological material would not hinder the fluorophore signal. However, because Si surfaces are easily oxidized, maintaining red emission from Si NPs dispersed in polar solvents has been challenging.

The most common methods for protecting the emission from red-emitting Si NPs have involved sufficient surface passivation with covalently bound surface groups, or by creating a molecular barrier. It has recently been shown that red-emitting decenefunctionalized Si NPs can be exposed to alcohols with chain lengths greater than four can still maintain their red emission. ${ }^{[97 e]}$ This is believed to occur through a "wedging" of the alcohols within the decane surface groups, thus preventing the $\mathrm{OH}$ - moiety from interacting with the Si NP surface. Additionally, preparing Si NPs within micelles by using surfactants ${ }^{[23,97 d]}$ creates an "umbrella" effect over the Si NP, making it difficult for small oxidizing molecules to reach the Si NP surface. In efforts to understand the efficiencies of these protection methods on the Si NP emission, red-emitting esterterminated Si NPs have been prepared from the thermal hydrosilylation (HS) of hydrideterminated Si NPs and bi-functional esters. These particles were subjected to straight chain alcohols in order to determine if the length of the ligand chain or the increase of steric hindrances can protect the Si surface from the alcohol solvents. 


\subsection{Si NP Experimental and Characterization}

Materials: All synthetic manipulations were performed under an inert (Ar) atmosphere using standard Schlenk techniques. 4-Pentenoyl chloride $\left(\mathrm{C}_{5} \mathrm{H}_{7} \mathrm{OCl}\right.$, Sigma, 98\%), 10undecenoyl chloride $\left(\mathrm{C}_{11} \mathrm{H}_{19} \mathrm{OCl}\right.$, Sigma, 97\%) and o-xylene $\left(\mathrm{C}_{6} \mathrm{H}_{4}\left(\mathrm{CH}_{3}\right)_{2}\right.$, Sigma Aldrich, $\geq 98 \%)$ were used as received. Methanol $\left(\mathrm{CH}_{4} \mathrm{O}\right.$, Sigma $\left.99 \%\right)$, tert-butyl alcohol $\left(\mathrm{C}_{4} \mathrm{H}_{10}\right.$, Acros, $\left.99.5 \%\right)$, pentanol $\left(\mathrm{C}_{5} \mathrm{H}_{12} \mathrm{O}\right.$, Sigma, $\left.97 \%\right)$, and decanol $\left(\mathrm{C}_{10} \mathrm{H}_{22} \mathrm{O}\right.$, Sigma, 97\%) were dried using $3 \AA$ molecular sieves prior to use.

\subsubsection{Synthesis of the bi-Functional Esters}

The bi-functional esters were prepared from the nucleophilic substitution of the appropriate acid chloride and alcohol.

Methylated esters: Methanol was slowly added to the corresponding acid chloride (4pentenoyl chloride or 10-undecenoyl chloride) in 1:10 stoichiometric ratios, and was stirred for $30 \mathrm{~min}$ at $25^{\circ} \mathrm{C}$ or $50{ }^{\circ} \mathrm{C}$, respectively. After cooling to room temperature, 40 $\mathrm{mL}$ of $10 \%$ sodium bicarbonate was added to extract the aqueous layer. The organic layer was dried with anhydrous magnesium sulfate and characterized using FTIR and

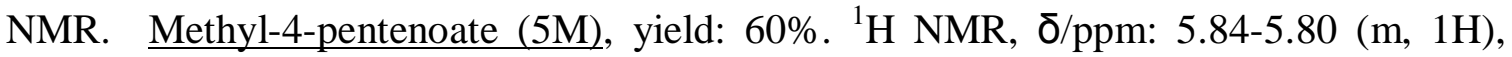
5.05-5.00 (m, 2H), $3.68(\mathrm{~s}, 3 \mathrm{H}), 2.42-2.38(\mathrm{~m}, 4 \mathrm{H}) ;{ }^{13} \mathrm{C} \mathrm{NMR}, \delta / \mathrm{ppm}(173.5,136.7$, 115.5, 51.5, 33.3, 28.8); Methyl-10-undecenoate (11M), yield: 70\%. ${ }^{1} \mathrm{H}$ NMR, $/ \mathrm{ppm}$ : (5.803-5.742 (m, 1H), 5.01-4.89 (m, 2H), $3.65(\mathrm{~s}, 3 \mathrm{H}), 2.31-2.29(\mathrm{~m}, 2 \mathrm{H}), 2.05-2.01(\mathrm{~m}$,

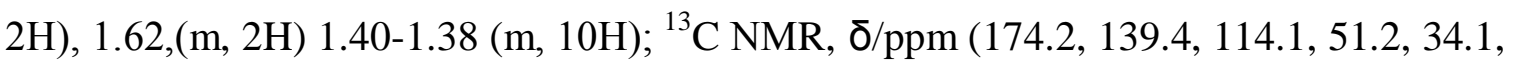
$33.8,29.34,29.26,29.19,29.11,28.9,25.0)$. 
Tert-butyl esters: tert-Butyl alcohol (heated to $40^{\circ} \mathrm{C}$ ) was added to the corresponding acid chloride (4-pentenoyl chloride or 10-undecenoyl chloride) in 1:10 stoichiometric ratios and stirred for $1 \mathrm{hr}$ at $40^{\circ}$ or $70^{\circ} \mathrm{C}$, respectively. When cooled to room temperature, $40 \mathrm{~mL}$ of $10 \%$ sodium bicarbonate was added to extract the aqueous layer. The organic layer was dried with anhydrous magnesium sulfate and characterized using FTIR and NMR. tert-Butyl-4-pentenoate (5T), yield: 80\%. ${ }^{1} \mathrm{H}$ NMR, $/$ /ppm: $(5.88-5.77$ (m, 1H), 5.07-4.92 (m, 2H), 2.34-2.32 (m, 4H), $1.45(\mathrm{~s}, 9 \mathrm{H}) ;{ }^{13} \mathrm{C}$ NMR $\delta / p p m ~(172.8$, 136.6, 115.1, 69.0, 34.8, 31.20, 28.11); tert-Butyl-10-decenoate (11T), yield: $85 \% .{ }^{1} \mathrm{H}$ NMR, ठ/ppm: (5.85-5.73 (m, 1H), 5.01-4.872 (m, 2H), 2.25-2.15 (m, 2H), 2.06-1.96 (m,

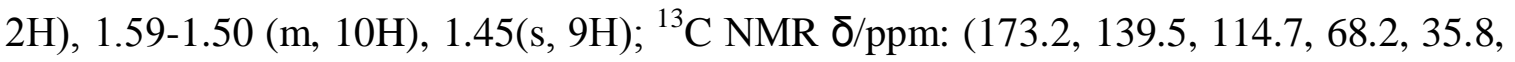
$34.4,31.5,30.4,30.2,29.4,29.2,28.1,25.7)$.

\subsubsection{Synthesis of Ester-Functionalized Si NPs}

The ester-functionalized Si NPs were prepared from the thermal hydrosilylation (HS) of hydride-terminated Si NPs (Si-H NPs) with the prepared bi-functional esters. The Si-H NPs were prepared from a previously described literature method. ${ }^{[141]}$ Briefly, $45 \mathrm{mmol}$ of trichlorosilane ( $\mathrm{HSiCl}_{3}$, Alfa Aesar 97\%) was polymerized using $90 \mathrm{mmol}$ of nanopure water $\left(\mathrm{nH}_{2} \mathrm{O}, 18 \mathrm{MOhm} \bullet \mathrm{cm}\right.$ resistivity). After drying for $24 \mathrm{hrs}$, the polymer was annealed at $1100{ }^{\circ} \mathrm{C}$ for $10 \mathrm{hrs}$. Nanocrystalline $\mathrm{Si}$, encapsulated in a silica matrix, were ball-milled for $10 \mathrm{~s}$ and etched using concentrated hydrofluoric acid (HF, Sigma, 47-52\%) ethanol $\left(\mathrm{C}_{2} \mathrm{H}_{6} \mathrm{O}\right.$, Sigma, anhydrous), and $\mathrm{nH}_{2} \mathrm{O}$ (volume 1:1:1). The chemical etch was quenched after $1 \mathrm{hr}$ by extracting the red-emitting Si-H NPs with pentane. 
In a typical thermal $\mathrm{HS}, 10 \mathrm{~mL}$ of freshly prepared $\mathrm{Si}-\mathrm{H}$ NPs in pentane were added to a two-neck round-bottomed flask on the Schlenk line. After evaporating the pentane, $3 \mathrm{~mL}$ of the bi-functional ester in $40 \mathrm{~mL}$ of o-xylene was added via cannula. The reaction was stirred overnight at $30{ }^{\circ} \mathrm{C}$ or $90{ }^{\circ} \mathrm{C}$ for the 5 -carbon or 11-carbon chained esters, respectively. o-Xylene and unreacted esters were removed using rotary evaporation. The ester-functionalized Si NPs were dispersed in hexane prior to characterization.

\subsubsection{Alcohol Dispersions of the Ester-Functionalized Si NPs}

The ester-synthesized Si NPs were suspended in alcohols of varying chain. Typically, $2 \mathrm{~mL}$ of the ester-synthesized Si NPs were dried in a standard scintillation vial using rotary evaporation. Once sufficiently dry, $2 \mathrm{~mL}$ of methanol, pentanol, or decanol was added to the Si NPs. The dispersion was sonicated for 2 minutes prior to optical characterization.

\subsubsection{Characterization of the bi-Functional Esters and Ester-Functionalized Si NPs}

Nuclear Magnetic Resonance (NMR) spectroscopy: ${ }^{1} \mathrm{H}$ and ${ }^{13} \mathrm{C}$ NMR patterns of the prepared esters were recorded in $\mathrm{CDCl}_{3}$ or $\left(\mathrm{CD}_{3}\right)_{2} \mathrm{CO}$ on a Bruker $400 \mathrm{mHz} \mathrm{NMR}$ spectrometer.

Fourier Transform Infrared (FT-IR) Spectroscopy: FT-IR spectra of the prepared esters and the ester-synthesized $\mathrm{Si}$ NPs were collected on a Nicolet iS10 spectrophotometer using a diamond ATR attachment using 64 scans with a resolution of $2 \mathrm{~cm}^{-1}$. 
Transmission Electron Microscopy (TEM): TEM measurements, performed on a Tecnai F-20 HR-TEM operating at $200 \mathrm{kV}$, were used to assess the morphology, size, size distribution, and composition of the Si NP samples. Samples were prepared by drop casting diluted aliquots of neat Si NPs in hexanes onto 400-mesh holey carbon-coated copper grids (SPI), which were subsequently dried at $100^{\circ} \mathrm{C}$ overnight.

$X$-Ray Diffraction (XRD): Powder XRD patterns were collected on a sample of the ester-synthesized Si NPs on a Rigaku Ultima IV X-Ray diffraction system with $\mathrm{Cu} \mathrm{K}_{\alpha}$ radiation $(\lambda=1.54187 \AA)$ in the $20-702 \theta$ range using a 2 second count time and $0.01^{\circ}$ step width.

Steady State Photoluminescence (PL) Spectroscopy: Steady State PL measurements were collected on a Shimadzu-RF5301PM spectrophotometer using a standard $1 \mathrm{~cm}$ quartz cuvette.

\subsection{Results and Discussion}

Hydrosilylation (HS) reactions have been shown to functionalize $\mathrm{Si}-\mathrm{H}$ surfaces with unsaturated hydrocarbons, including, alkenes, esters, and amines. [20a-c, 21b, 25b, 93d, 96, 109-110, 124a, 147] However, a majority of these studies have focused on preparing and analyzing Si NPs with straight chained alkenes dispersed in nonpolar media; ${ }^{[20 b, 21 b, 109 a,}$ 124a, 147] these ligands have been shown to have little effect on Si NP emission. Recently, red-emitting decene-functionalized Si NPs were found to be affected by the presence of short chain alcohols (e.g., methanol). ${ }^{[97 e]}$ As a solvent, methanol was been shown to oxidize the Si NP surface, and heavily blue shift the Si NP emission. ${ }^{[97 c, 97 e]}$ However, 
longer change alcohols may be "wedged" within the decane terminal groups, and are not able to reach or oxidize the NP surface. Additionally, micelles have been shown to act as a molecular barrier, or "umbrella", to the Si NP surface with steric hindrances that also protect the Si NP surface (and the observed photophysical properties) from unintended change. However, these "umbrella" complexes involve multiple synthetic steps, and often generate Si NPs larger than $25 \mathrm{~nm}$ hydrodynamic diameters. To determine if steric hindrances to small molecular oxidants (e.g., $\mathrm{OH}-$ ) can be directly functionalized to the NP surface, ester-functionalized Si NPs were prepared by the HS of Si-H NPs with bifunctional esters (Figure 7.1). The esters ligands were prepared from the nucleophilic substitution of the corresponding acid chloride with methanol or tert-butanol, with the goal of determining if the chain length of the hydrocarbon or the steric hindrance of the terminating ester moiety could best preserve the red-emission from the Si NPs.

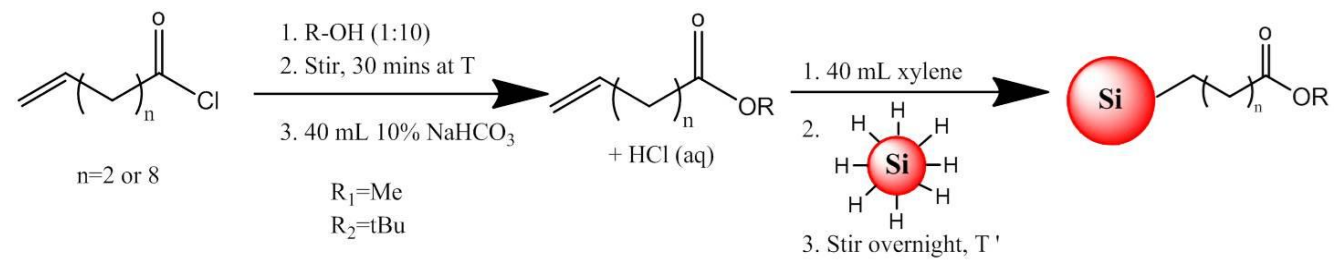

Figure 7.1. Scheme of the synthesis of the bi-functional esters and the ester-synthesized Si NPs. The nucleophilic substitution of the corresponding acid chloride (where $n=2$ or 8 ) with both $\mathrm{R}_{1,2} \mathrm{OH}$ (for $\mathrm{n}=2$, $\mathrm{T}=25{ }^{\circ} \mathrm{C}$; for $\mathrm{n}=8, \mathrm{~T}=50{ }^{\circ} \mathrm{C}$ ). The thermal HS of the obtained bi-functional esters and Si-H NPs was performed at $\mathrm{T}^{\prime}=30^{\circ} \mathrm{C}$, or $90^{\circ} \mathrm{C}$, for $\mathrm{n}=2$ and $\mathrm{n}=8$, respectively. 


\subsubsection{Analytical Characterization of the Ester-Functionalized Si NPs}

The ester-functionalized Si NPs were characterized by FT-IR (Figure 7.2), which clearly reveals stretches consistent with ester functionalization, including a carbonyl vibration at about $1740 \mathrm{~cm}^{-1}$. The methyloxy and tert-butyloxy stretches are observed at about $1197 \mathrm{~cm}^{-1}$, respectively, in addition to other $\mathrm{C}-\mathrm{H}$ stretching signals, consistent with the presence of the ester (See full spectra for the ester-functionalized Si NPs in Appendix). The FT-IR of all the ester-synthesized Si NPs shows evidence for the formation of covalent Si-C stretches at about $1260 \mathrm{~cm}^{-1}$ (marked with *), consistent with a successful HS. The ester-synthesized Si NPs, with the exception of the methyl-4pentenoate-Si NPs (5M-Si NPs), showed some Si-O passivation at 1000 and $800 \mathrm{~cm}^{-1}$ (highlighted).

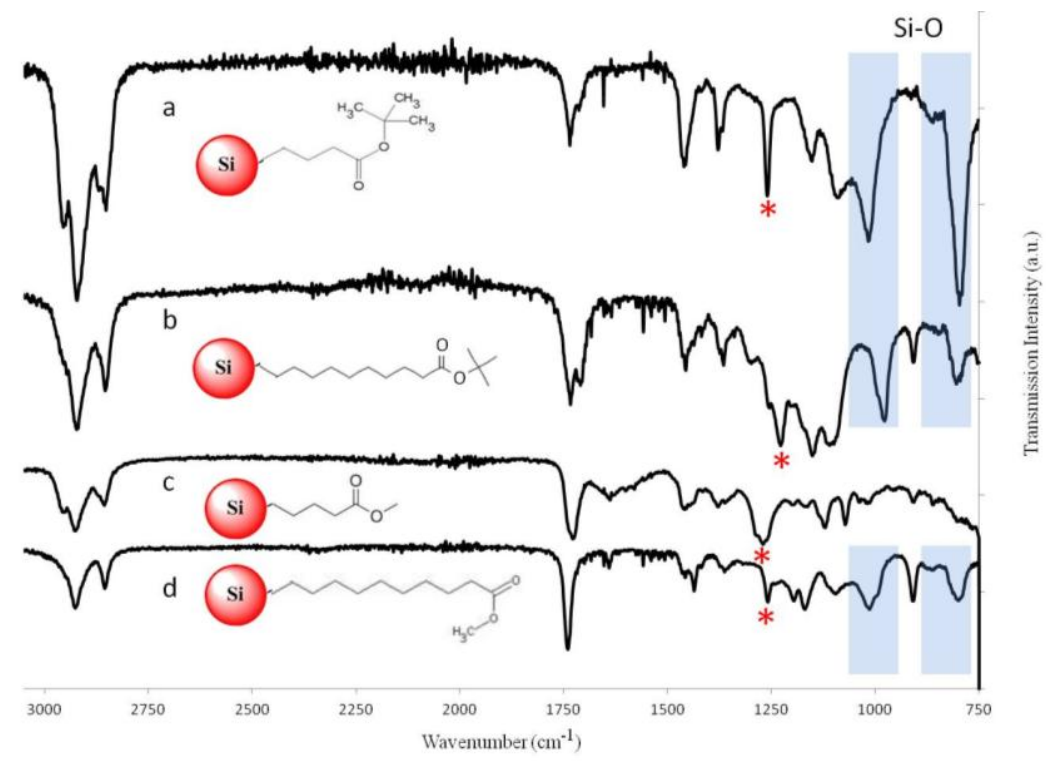

Figure. 7.2. FTIR spectra of (a) tert-butyl-4-pentenoate- (5T), (b) tert-butyl-10-decenoate- (11T), (c) methyl-4-pentenoate- (5M), and (d) methyl-10-undecenoate (11M)-Si NPs. The Si-C stretch (marked with *) supports the successful HS of the Si-H NPs with bifunctional esters alongside some surface oxide. 
Representative TEM images of the ester-functionalized Si NPs (methyl-10decenoate-Si NPs, 11M-Si NPs) are shown in Figure 7.3. All of the ester-functionalized Si NPs were found to be spherical, well-isolated NPs (See Appendix for complete TEM images). The cores were found to be crystalline with obvious lattice fringes. SAED patterns on the NPs found varying lattice planes (including the (111), (220), and (400)) consistent with $\mathrm{Si}^{0}$ (JCPDS card no 001-0787). The average size did not vary much with the terminating ester groups; as shown in Table 7.1, the ester-terminated Si NPs were found to have an average size of $5.51 \pm 1.35 \mathrm{~nm}$.

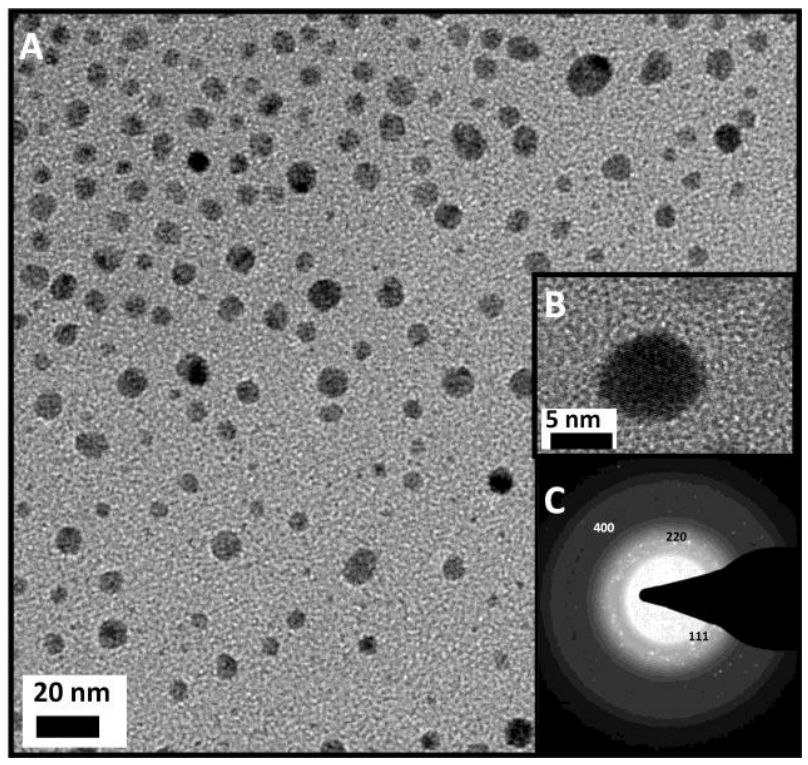

Figure 7.3. Representative TEM images of the 11M-Si NPs illustrating the morphology and crystallinity (A.,B.); The SAED pattern supports the presence of the (111), (220), and (400) diffraction planes (C.). 
Table 7.1. Si NP size and SAED diffraction patterns from the ester-functionalized Si NPs

\begin{tabular}{ccc}
\hline Ester coating & Size $(\mathrm{nm})$ & $\mathrm{Si}^{0}$ Diffraction Planes \\
\hline methyl-4-pentenoate & $5.83 \pm 1.40$ & $(111)$ \\
methyl-10-decenoate & $5.16 \pm 1.22$ & $(111),(220),\left(111^{*}\right)$ \\
tert-butyl-4-pentenoate & $5.42 \pm 1.31$ & $(111),(220)$ \\
tert-butyl-10-decenoate & $5.60 \pm 1.45$ & $(111),(220),(400)$ \\
\hline
\end{tabular}

$\mathrm{Si}^{0} ;$ JCPDS card no. 001-0787

${ }^{*} \mathrm{SiO}_{2}$; JCPDS card no. 087-2096

Solids began flocculating from the $11 \mathrm{M}-\mathrm{Si}$ NP solution in hexane soon after synthesis. These powders were collected and analyzed via XRD to determine if the functionalized Si NPs were $\mathrm{Si}^{0}$. As shown in Figure 7.4, the XRD pattern of the $11 \mathrm{M}-\mathrm{Si}$ NPs compared to a calculated $\mathrm{Si}^{0}$ PXRD pattern (JCPDS card no. 001-0787 shows the observed NP peaks are consistent with the (100), (200), and (311) reflections of the $\mathrm{Si}^{0}$ pattern. This indicates that the particles consist of crystalline $\mathrm{Si}$. It should be noted that the Si NP peaks from the obtained XRD pattern are sharper than expected for NPs. It is generally anticipated that decreasing particle size often results in peak broadening. ${ }^{[148]}$ The fact that the XRD spectra of the Si NPs do not exhibit significant broadening may be due to the agglomeration of the NPs, which caused their initial flocculation. 


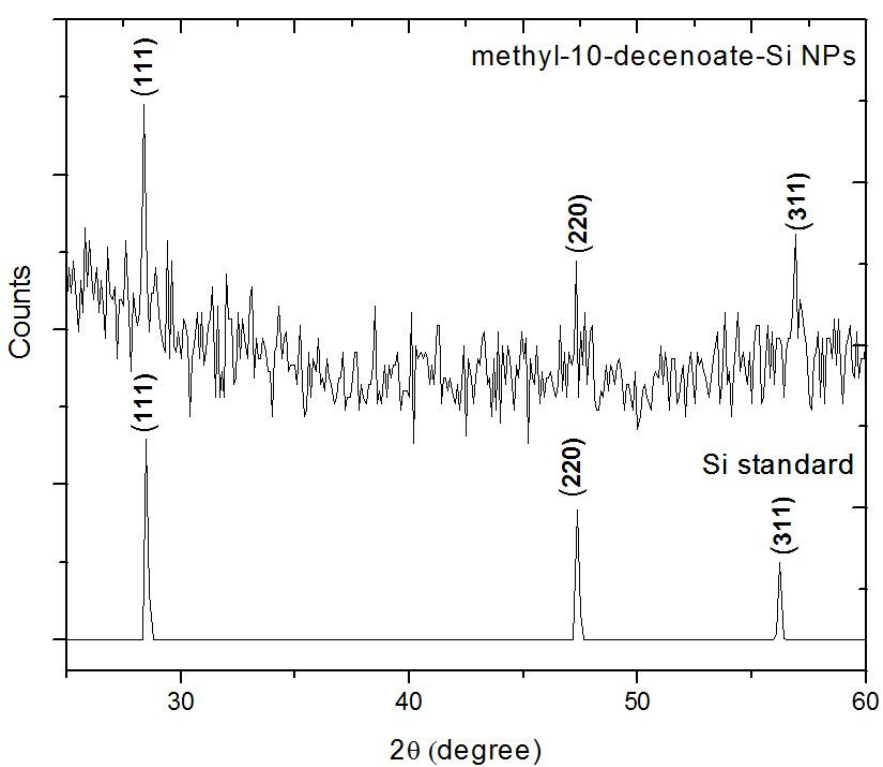

Figure 7.4. PXRD pattern of the 11M-Si NPs (top) compared to the calculated PXRD pattern of diamond lattice Si (bottom, JCPDS card no. 001-0787). The (100), (200), and (311) reflections are consistent between the Si NP and Si standard patterns, indicating the flocculated particles correspond to crystalline Si.

Sample UV-Vis absorption spectra of the ester-functionalized Si NPs are shown in Figure 7.5. The absorption spectra of both the methyl-4-pentenoate (5-M) and tertbutyl-10-undecenoate (11T) Si NPs show an absorption onset at approximately $500 \mathrm{~nm}$ with a distinct shoulder that peaks at about $295 \mathrm{~nm}$. The long absorption tail is consistent with the indirect bandgap character of $\mathrm{Si}$ while the shoulder at $295 \mathrm{~nm}(4.2 \mathrm{eV})$ has been associated with potential direct bandgap transitions in $\mathrm{Si}^{[4,20 \mathrm{~d}, 24]}$ 


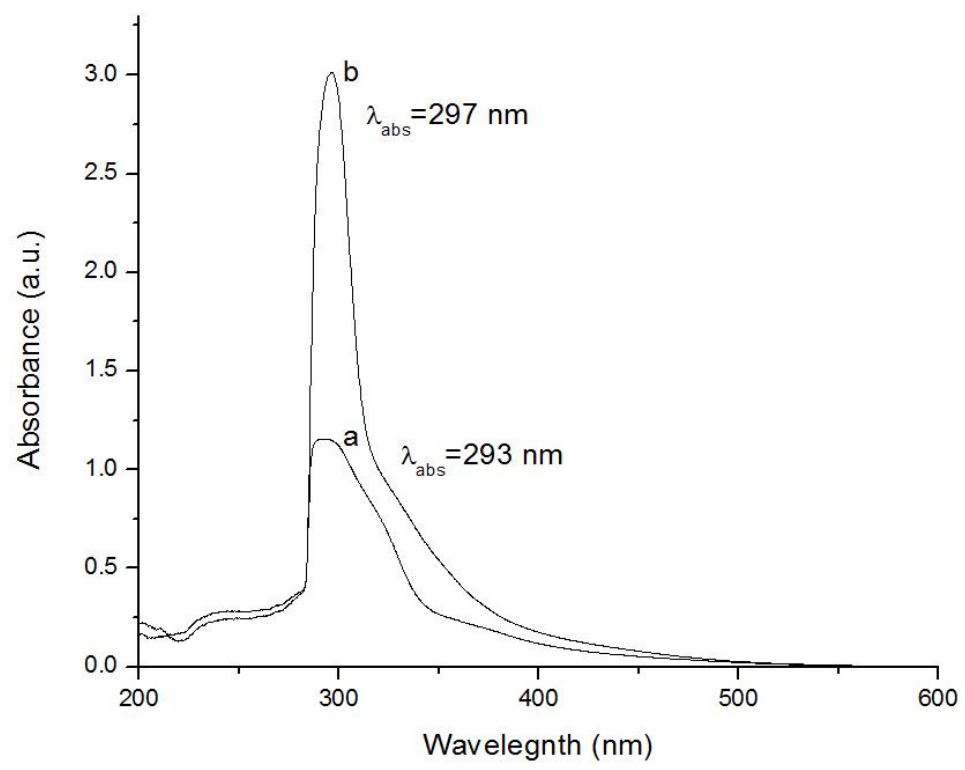

Figure 7.5. Representative UV-Vis absorption spectra of the ester-synthesized Si NPs. The absorption spectra of (A) 5M-Si NPs, and (B) 11T-Si NPs with an absorbance onset at $500 \mathrm{~nm}$ and shoulders at $\sim 295$ nm.

The precursor Si-H NPs (dispersed in pentane) have an emission maximum at 600 nm (Figure 7.6) with a red/orange emission observed by eye using a hand held UV lamp. After the HS with the bi-functional esters, the Si NP solutions still exhibited an observed red/orange color. Using 340-460 $\mathrm{nm}$ excitation wavelengths, both blue and red emissive components were observed. The blue component $\left(\sim \lambda_{\max }=450\right.$ or $525 \mathrm{~nm}$, Table 7.1$)$ was found to shift as a function of excitation energy. All emission spectra also have a red component, or shoulder at $\sim 570 \mathrm{~nm}$, which showed no excitation dependence. 


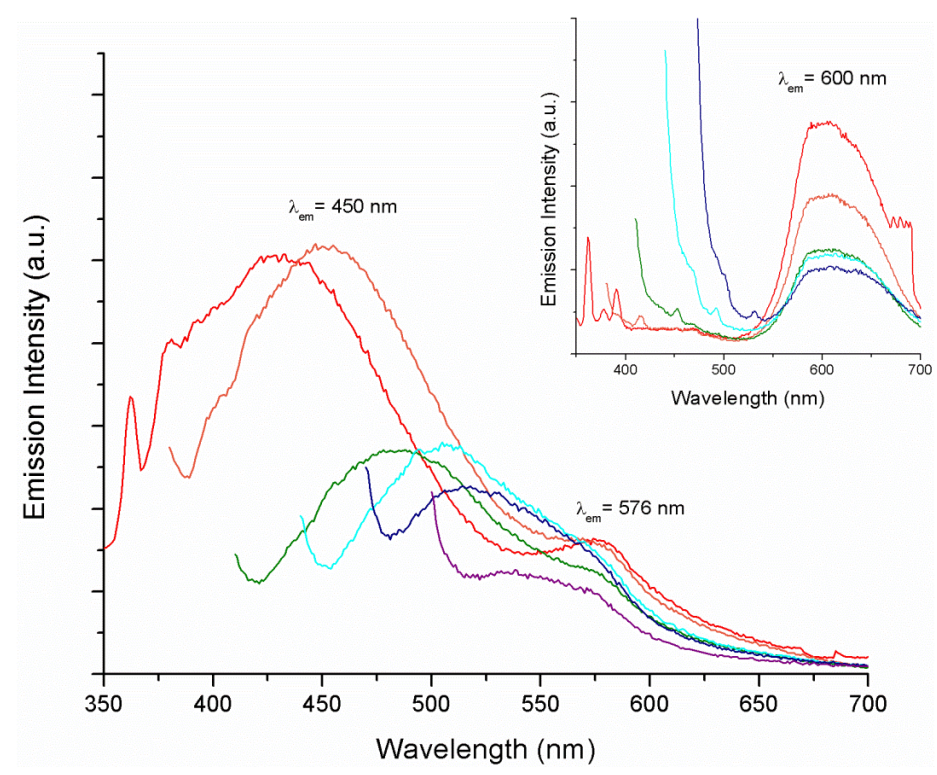

Figure 7.6. Representative emission spectrum of the 5M-Si NPs. Various excitation wavelengths were used (shown 340-4 $90 \mathrm{~nm}$, in $30 \mathrm{~nm}$ intervals). Both a blue emission component and a red emission component are observed; Inset. Si-H NP emission spectrum using various excitation wavelengths (340-460 $\mathrm{nm}$, in $30 \mathrm{~nm}$ intervals).

Table 7.2. Emission Maxima ( $\lambda_{\mathrm{ex}}=370 \mathrm{~nm}$, unless noted $)$ of the ester-functionalized Si NPs

\begin{tabular}{ccccc}
\hline Ester Termination & o-xylene & methanol & pentanol & decanol \\
\hline & $\lambda_{\max }(\mathrm{nm}) /$ & $\lambda_{\max }(\mathrm{nm}) /$ & $\lambda_{\max }(\mathrm{nm}) /$ & $\lambda_{\max }(\mathrm{nm}) /$ \\
observed color & observed color & observed color & observed color \\
methyl-4-pentenoate & $450 / 576 /$ orange & $530 / \mathrm{blue}$ & $468 / \mathrm{blue}$ & $430 / \mathrm{blue}{ }^{\#}$ \\
methyl-10-decenoate & $450 / 576 /$ orange & $450 / \mathrm{blue}$ & $448 / 560 /$ turbid & $449 / 565 /$ orange \\
tert-butyl-4-pentenoate & $440 / 574 /$ orange & $463 / 563 /$ orange & $462 / 528 /$ orange & $461 / 565 /$ orange \\
tert-butyl-10-decenoate & $525 / 567 /$ orange & & & \\
${ }^{\wedge} \lambda_{\text {ex }}=460 \mathrm{~nm}$ & & & \\
${ }^{\#} \lambda_{\mathrm{ex}}=340 \mathrm{~nm}$ & & & & \\
& & &
\end{tabular}

\subsubsection{Photophysical Study by Alcohol Dispersions}

The origin of emission from nanosized Si could be due to the possibility of both radiative exciton recombination events from Si NP cores, as well as from localized states arising from surface, interfacial, or surface defect-related recombination. ${ }^{[4]}$ While Si-H NPs have been shown to possess size-tunable emission profiles, upon oxidation, 
unexpected emission shifts have been observed. Therefore, discriminating between radiative recombination events from the core and surface/defect sites is complicated.

Because others have shown that both the surface chain length and bulk wrapping have, to some extent, been found to preserve red emission from Si NPs, both of these protection schemes are varied here. The ester-synthesized Si NPs were exposed to short (methanol), medium (pentanol), and long (decanol) chain alcohols, which were found to effect the emission differently. For example, when the5M-Si NPs were exposed to alcohols, the observed emission color immediately changed from red/orange to blue (Table 7.2). While the solutions appear blue, the emission spectra do not drastically change (Figure 7.7). However as the alcohol chain increases, there is more significant blue shifting in the emission spectra. This may be consistent with increased surface oxidation, but requires further analysis to confirm. 
A

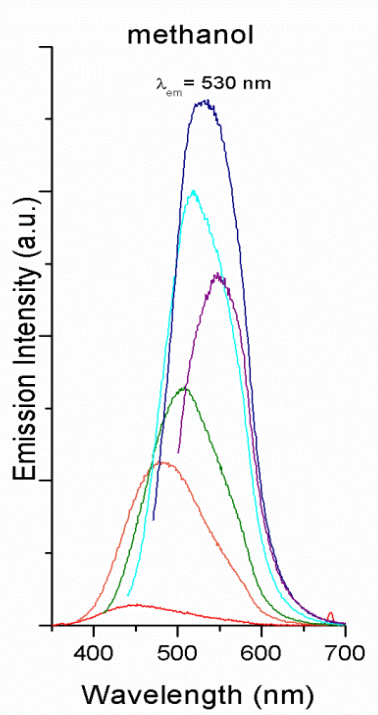

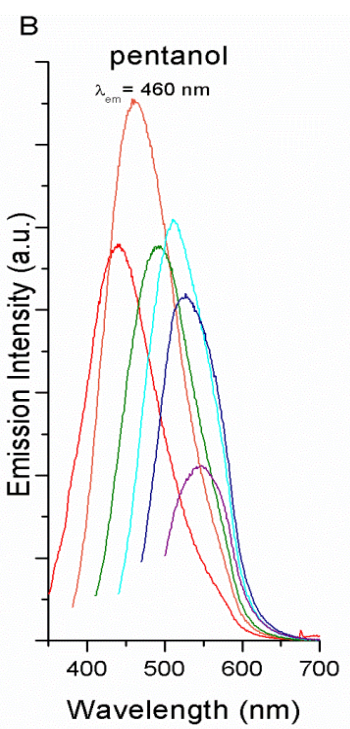

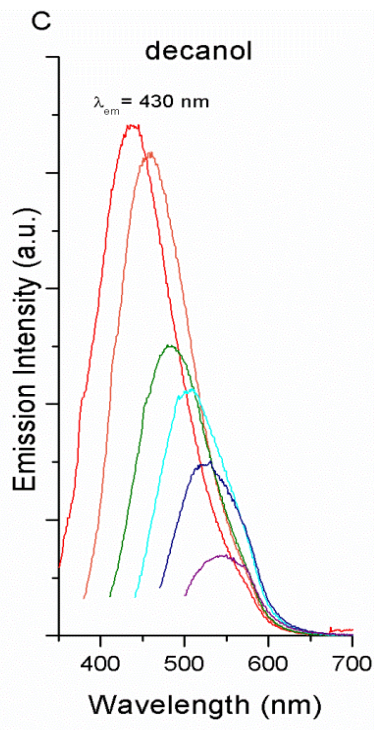

Figure 7.7. Emission spectra of 5M-Si NPs dispersed in methanol, pentanol, and decanol using various excitation wavelengths (340-490, in $30 \mathrm{~nm}$ intervals). The emission maximum exhibits and increasing blue shift with increasing alcohol chain length.

When the longer, methyl-10-decenoate-Si NPs (11M-Si NPs) were exposed to alcohols, slightly different effects on the emission spectra as compared to the methyl-4pentenoate-Si NPs were observed. In methanol (Figure 7.8) the 11M-Si NP solution emission immediately changed to blue (by eye). The emission spectra supports this observation, with a new maximum emission at $450 \mathrm{~nm}\left(\lambda_{\mathrm{ex}}=370 \mathrm{~nm}\right.$, Table 7.2). When exposed to pentanol, the solution was turbid and cloudy, but was still found to have blue emission $\left(\lambda_{\max }=448 \mathrm{~nm}\right)$ with a red shoulder $\left(\lambda_{\mathrm{em}}=560 \mathrm{~nm}\right.$, Figure 7.8). In the presence of decanol, however, the methyl-10-decenoate-Si NP solution remained orange. Unfortunately, the emission changed to blue after $24 \mathrm{hrs}$, even while possessing a red shoulder at about $565 \mathrm{~nm}$ (Figure 7.8, Table 7.2). 

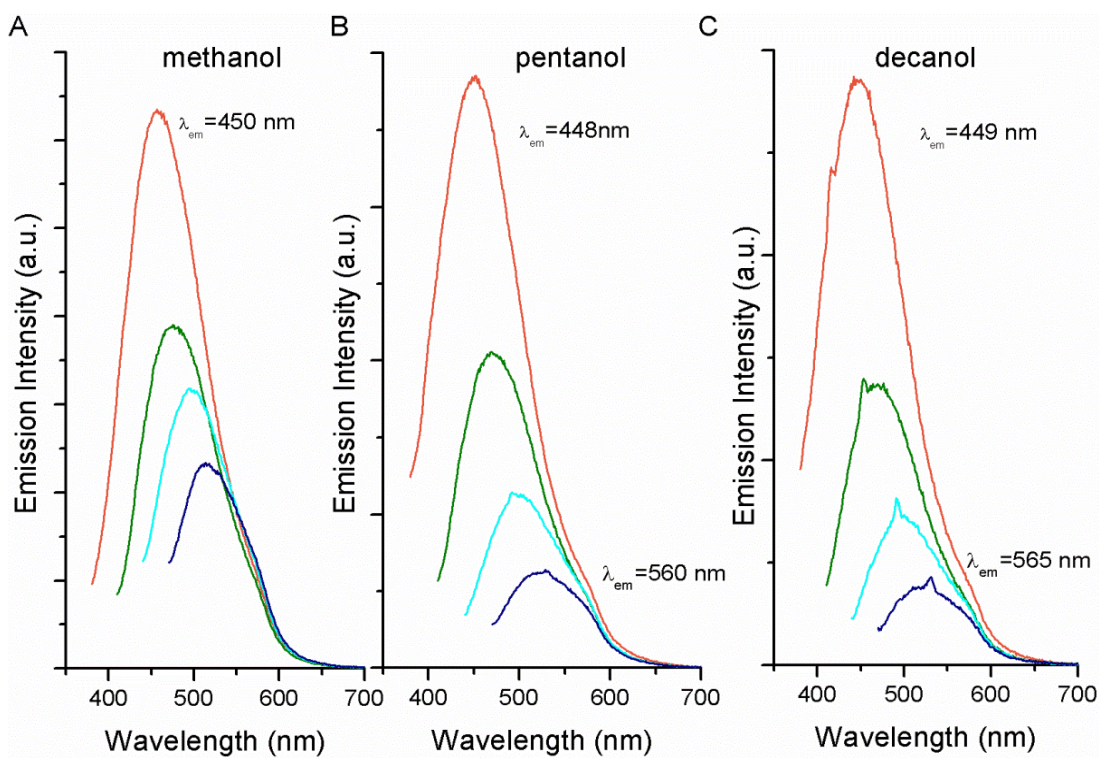

Figure 7.8. Emission spectra of 11M-Si NPs dispersed in methanol, pentanol, and decanol using various excitation wavelengths (370-460, in $30 \mathrm{~nm}$ intervals). All spectra have similar spectra, but the pentanol and decanol dispersions still have a red shoulder at 560 and $565 \mathrm{~nm}$, respectively.

The bulky tert-butyl-5-pentenoate-Si NPs (5T-Si NPs) exposed to the alcohols had no emission change; all the samples remained orange. This was supported in the emission spectra (Figure 7.9), as all the dispersions possessed almost identical emission spectra. While an increase in the intensity of the blue emission (as compared to the 5T-Si NPs in xylene), there is little change to the red emission component (at about $565 \mathrm{~nm}$ ). This suggests that the bulky tert-butyl group is able to prevent both large and small alcohol molecules from reaching the surface of the Si NPs, which may be responsible for the blue shifted emission. Other surface techniques, such as FT-IR or X-ray Photoelectron Spectroscopy, would be needed to confirm this. 

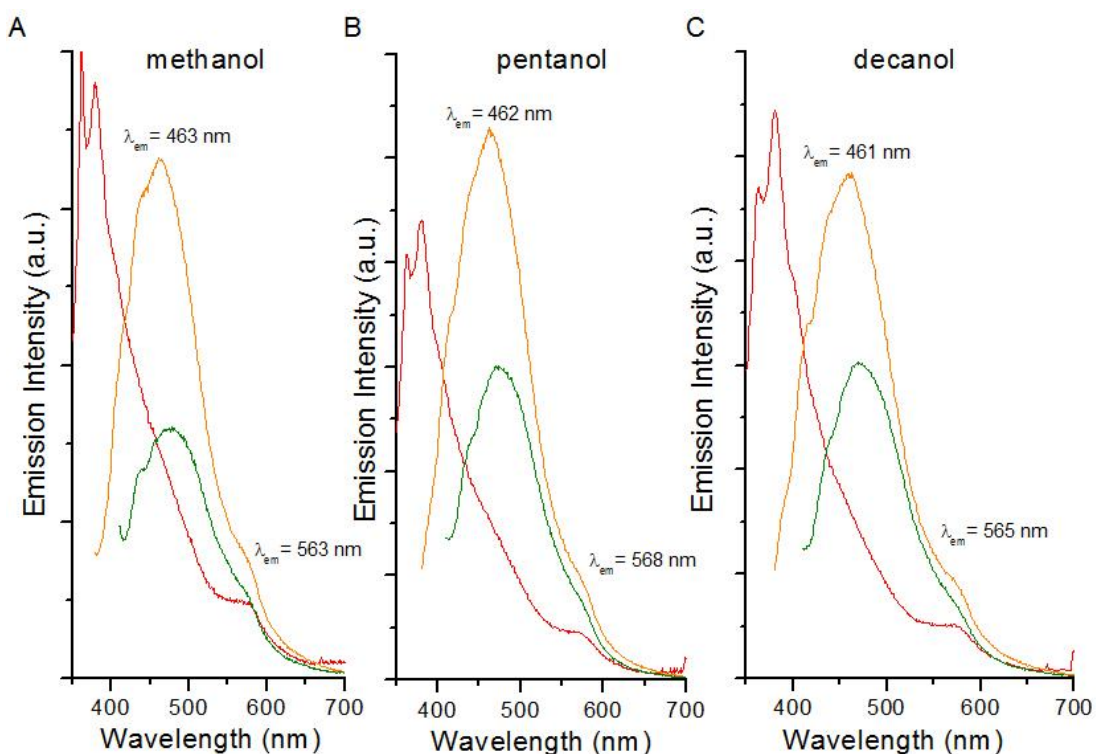

Figure 7.9. Emission spectra of tert-butyl-4-pentoate-Si NPs dispersed in methanol, pentanol, and decanol using various excitation wavelengths (340-400, in $30 \mathrm{~nm}$ intervals). The emission spectra of all of the dispersions look similar, still containing a blue and red emission competent.

While the tert-butyl-10-decenoate-Si NPs (11T-Si NPs) have yet to be dispersed in various alcohol molecules, it is hypothesized that the red emission will also be preserved due to the bulky tert-butyl group.

\subsection{Conclusions}

The origin of nanosized Si emission has been debated, as both particle size alongside surface-related effects have been found to cause shifts in the Si nanoparticle (NP) emission. Regardless of the origin of Si NP emission, functional surface groups are used to terminate the NPs with substituents that are useful and capable of preserving the desired optical properties. It has been found that increasing the surface passivation on $\mathrm{Si}$ NPs or utilizing molecular barriers have protected the Si NP surface from oxidation. The 
thermal hydrosilylation of hydride-terminated Si NPs with bi-functional esters of different hydrocarbon chain lengths (5 and 11-carbon) and different ester terminations (methyl and tert-butyl) have produced ester-functionalized Si NPs. To test whether chain length or steric hindrance of the ligand better protect the Si NP surface from interacting with straight chain alcohol solvents, the red-emitting $\left(\lambda_{\mathrm{em}}=575 \mathrm{~nm}\right)$ ester-functionalized Si NPs were dispersed in methanol, pentanol, and decanol. When the methoxy esterfunctionalized Si NPs were exposed to the alcohols, the observed emission changed to blue, which occurred immediately in the presence of methanol. However, when the tertbutyl-4-pentoate-Si NPs were exposed to the alcohols, the red emission is maintained. This suggests that the barrier method of protecting the Si NP surface may be more efficient than increased surface coverage. 


\title{
General Summary, Discussion, and Future Work
}

\begin{abstract}
This chapter gives an overview of the research results presented in this dissertation. Personal views and ideas for further research directions related to the projects of this dissertation and discussed.
\end{abstract}




\subsection{Summary}

Nanoscience and nanotechnology blend the concepts of physics, chemistry, biology, and engineering with the intention of understanding and utilizing materials in the 1-100 nm size range. While nanomaterials have been used over thousands of years, it is only in the last three decades that research into semiconductor nanomaterials has focused on optimizing the properties of quantum dots (QDs) and nanoparticles (NPs). The ability to control the unique photophysical properties of NPs makes them attractive for many new applications. The size-tunable emission originates from their intermediate size between that of bulk material and molecular structures. The decrease of bulk semiconductors to the nanosize that confines electrons to discrete energy levels rather than in continuous energy bands is known as the quantum confinement (QC) effect. As the particle becomes smaller, this confinement appears to affect the magnitude of the bandgap, giving NPs size-tunable emission properties. This size tunable emission of semiconductor NPs, in addition to their broad absorption and narrow emission spectra, high quantum yields from the UV-NIR, and significant resistance to photobleaching makes them advantageous for light emitting applications, as compared to organic dyes, specifically as it pertains to potential biomedical imaging and sensing. CdSe-based QDs have been the most studied, as they were one of the first NPs in which a full series of size-controlled, monodisperse particles were produced. Even though these QDs have been prepared with benign semiconductor shells, their inherent toxicity has led to the interest in developing other nontoxic NPs for biomedical applications. 
The indirect bandgap nature of $\mathrm{Si}$ has previously restricted its applications to electronics; the absorption of a phonon in addition to a photon leads to inefficient absorption and emission from bulk Si. However, the observation of efficient photoluminescence (PL) from nanosized $\mathrm{Si}$ has expanded its potential application to include visible light emitters. Si NPs have similar photophysical properties to CdSebased QDs, though the origin is not yet fully understood. In addition to QC, the presence of surface states (e.g., incomplete bonds, surface defects) may also act as nonradiative or radiative centers, which can generate unexpected shifts in emission (Chapters 1 and 2).

The goals of this dissertation, include: 1) the development of emissive, photostable Si NPs; 2) the photophysical characterization of Si NPs, and determine how the surface can influence the observed emission characterizations; 3) to gain insight into the mechanism and applications of direct and indirect semiconductor NPs by observing their emission quenching in the presence of analytes.

Chapter 3 describes the mechanistic examination of the serotonin (5hydroxytryptamine, 5-HT) concentration-dependent decrease in CdSe/ZnS QD emission intensity in untargeted, ensemble-QD and receptor-targeted, single-QD studies. Energy transfer, as well as core and/or shell degradation involving 5-HT were investigated, and were eliminated as possible sources for QD intensity loss. However, surface reconstruction, by the dynamic loss of stabilizing ligands occurs on the surface of the QDs, when exposed to UV light regardless of the absence or presence of 5-HT. Timeresolved photoluminescence spectroscopy data reveals a decreasing QD lifetime with increasing 5-HT concentration, which is indicative of a faster exciton decay pathway, 
likely the result of a photoinduced charge transfer between the QDs and 5-HT. The dynamic loss of TOPO ligands allows 5-HT to coordinate to the QD, which presents the opportunity for the charge transfer between 5-HT and the QDs. The promise of using single QD nanosensors to detect 5-HT is also expanded upon by monitoring the emission from single QDs to detect down to $10 \mathrm{nM}$ 5-HT in a biological environment.

Chapter 4 aims at understanding the role of surface chemistry on Si NP emission. However, this become difficult when the Zintl salt metathesis between $\mathrm{NaSi}$ and $\mathrm{NH}_{4} \mathrm{Br}$ simultaneously produced hydrophilic and hydrophobic Si NPs upon thermal HS with 1decene in the presence of the coordinating solvent, N,N'-dimethylformamide (DMF). Both the hydrophobic and hydrophilic Si NPs show evidence for the surface coordination and termination of solvent molecules. Because all of the obtained Si NPs were approximately $5 \mathrm{~nm}$, the observed effects on the emission were not associated with QC, but rather with changes in the surface chemistry. Interestingly, the hydrophilic Si NPs prepared from the thermal and chemical initiated HS reactions had almost identical emission spectra, but relatively different surface chemistries (via FT-IR), though both were consistent with the presence of the solvent, DMF. The Zintl salt metathesis was also carried out in the non-coordinating solvent, o-xylene, and produced Si NPs successfully terminated with 1-decene, though they were weakly emissive. It appears as though the coordinating solvent plays a vital role in the formation of the Si NPs, or subsequent HS, and is, therefore, required.

Because the coordinating solvent appears to be a necessary component in the formation or functionalization of the Si NPs in the Zintl salt metathesis, Chapter 5 
describes the one-step, Zintl salt metathesis using bi-functional solvent ligands. It was anticipated that using di-carboxylic acids or di-amines as solvent ligands would allow for efficient stabilization of the particles as well as functionalize the Si surface. The one-step method consistently produced water-soluble, emissive Si NPs with partial surface oxidation, but also with surface-bound solvent molecules, including glutaric acid (GA), citric acid (CA), or ethylenediamine (en). TEM studies indicate that this one-step method produces small, monodisperse NPs with little dependence on the identity of the ligand solvent. These Si NPs were also blue emitting with emission lifetime decays 3-4 ns range. While the emission mechanism of these Si NPs may originate from radiative recombination of quantum confined excitons across the bandgap or from surface defects, the ease and versatility of the synthesis, the long-term aqueous stability of the Si NP products, and the extreme robustness of the photophysical properties make these Si NPs excellent candidates for imaging applications, which was demonstrate by imaging the GA-synthesized NPs in N2a cells.

In order to gain insight into the GA-Si NPs band edge, Chapter 6 describes a photophysical study in which the GA-Si NPs were exposed to metal salts. This interaction affected not only the emission spectra of the Si NPs, but also the absorption spectra of the metal cations. When $\mathrm{HAuCl}_{4}$ and $\mathrm{AgNO}_{3}$ were added to the GA-Si NPs, a decrease in the Si NP emission occurred, alongside an increased surface plasmon resonance band (SPR), consistent with the formation of $\mathrm{Au}^{0}$ and $\mathrm{Ag}^{0} \mathrm{NPs}$, respectively. Based on the reduction potentials of the metal ions and bulk $\mathrm{Si}^{0}$, the reaction between $\mathrm{AuCl}_{4}{ }^{-}$and bulk $\mathrm{Si}^{0}$ should produce $\mathrm{Au}^{0}$ spontaneously, while the reaction between $\mathrm{Ag}^{+}$ 
and bulk $\mathrm{Si}^{0}$ is not spontaneous. Because the GA-Si NPs are able to reduce both $\mathrm{AuCl}_{4}{ }^{-}$ and $\mathrm{Ag}^{+}$to $\mathrm{Ag}^{0}$, the bandgap, and specifically the reduction potential are likely influenced by QC. This interaction was compared to red-emitting $\mathrm{SiO}_{\mathrm{x}} / \mathrm{H}_{\mathrm{y}}-\mathrm{Si} \mathrm{NPs}\left(\mathrm{SiO}_{\mathrm{x}}-\mathrm{Si} \mathrm{NPs}\right)$, which were able to reduce $\mathrm{AuCl}_{4}{ }^{-}$, based on the formation of an SPR band and Au NPs via TEM. However, Ag NPs were not observed. The reduction potential of these particles may not bas significantly affected by QC, as compared to the GA-Si NPs, which may explain its inability to reduce $\mathrm{Ag}^{+}$.

While some assessments about the origin of Si NP emission can be made, the preservation of red emission from Si NPs is still imperative to their potential biological application. Therefore, ligands of varying chain lengths and steric modalities were used to protect ester-synthesized Si NPs when exposed to alcohol solvents (Chapter 7). Different hydrocarbon chain lengths (5 and 11-carbon) and different ester terminations (methyl and tert-butyl) were used to determine whether shorter chain length or increased steric hindrances can better protect the Si NP surface. The red-emitting $\left(\lambda_{\mathrm{em}}=575 \mathrm{~nm}\right)$ ester-functionalized Si NPs were dispersed in methanol, pentanol, and decanol. Both the short and long chain methoxy ester-functionalized Si NPs exhibited a blue emission when exposed to the alcohols. However, when tert-butyl-4-pentoate-Si NPs were exposed to the alcohols, the red emission is maintained. This suggests that the barrier method by way of significant steric hindrance may protect the Si NP surface more efficiently than increased ligand chain length. 


\subsection{General Discussion and Future Prospects}

The results described herein present a strong basis for understanding the emission properties of Si NPs. The results describe the synthesis of the Zintl salt metathesis between $\mathrm{NaSi}$ and $\mathrm{NH}_{4} \mathrm{Br}$ to produce hydrophobic and hydrophilic Si NPs. While these syntheses produced blue emitting particles, they still allow the effects of surface chemistry to be considered along the size effects, specifically those particles with carboxylic acid functionality that allows them to be imaged using N2A cells. However, different surfaces have been shown to protect red Si NP emission in alcoholic solutions, and this gives hope to developing red emitting, water-soluble Si NPs for biomedical imaging and sensing applications. However, before these particles can become more widely used as biological probes, several issues must be addressed

Many properties are useful for efficient biological fluorophores, and some of the more important properties include, 1) the observed emission range, 2) the quantum efficiency, or quantum yield (QY), 3) the fluorophore stability, 4) and the fluorophore functionality. An ideal biological fluorophore would possess emission in the 650-1200 $\mathrm{nm}$ range. Because few biological materials emit here, these fluorophores may have an undisrupted detection handle. In this emission region however, $\mathrm{Cd}$ - and $\mathrm{Pb}$-based QDs have reigned because the development of red-emitting, water-soluble, Si NPs has been challenging. Of the few published studies on red-emitting, water-soluble Si NPs, the Si surface is protected using bulky ligands or molecular barriers. In addition to using large ligands or polymers, the red emission of Si may also be protected using inorganic shells, such as $\mathrm{SiO}_{2}, \mathrm{ZnS}$, or $\mathrm{Au}$. These shells may protect the Si NP surface from undesired 
interactions in ensemble NP solutions, or during in vitro or in vivo experiments. In addition to protecting the observed emission of the Si NPs, surface capping or shells may also passivate surface defect sites, which can increase the QY. Si NPs have already been produced with an adequate QY for biological detection ( $10 \%)$, but further increasing it would continue to warrant their biological application.

The stability of a fluorophore refers not only to their physical robustness, but also to their photostability; emissive NPs cannot leach ions over time, and therefore the fate of the NPs must be studied. Relatively few reports have addressed the interaction of Si NPs with biological analytes. The study in Chapter 3 deals with the CdSe/ZnS QD emission quenching in the presence of the neurotransmitter serotonin (5-HT). However, other neurotransmitters and chemical sensor molecules may also affect the emission intensities of nanosensors QDs, and should be examined. While the photophysical study of the GASi NPs (Chapter 6) uses metal analytes to quench the Si emission, there are other biological relevant ions to investigate, including $\mathrm{Cu}^{2+}, \mathrm{Fe}^{2+}$, and $\mathrm{Zn}^{2+}$. Not only could the addition of more analytes give insight into the bandgap edges of the GA-Si NPs, but it may also give information as to the range of analytes that may be sensed using Si NP emission.

The overall focus of Si NPs needs to be the control of the emission properties. To do so, new synthetic methods to give precise control over both the Si NP size and surface termination are needed. Until such time, the aforementioned techniques to protect the NP surface may provide the necessary protection for the observed emission, but other spectroscopy techniques are likely required to determine the mechanism(s) of $\mathrm{Si}$ 
emission. Single-particle spectroscopy may give insight into the emission or surface termination of individual NPs rather than observing ensemble of NPs in solution. Additionally, time-resolved spectroscopy, as well as transient absorption spectroscopy, may allow the blue and red emission components of Si NP emission to be characterized independently. It is expected that the excited state lifetimes originating from exciton recombination across the bandgap will exhibit a size dependent trend, while the excited states originating from defects may not. Both the excited state lifetimes (size or surface related) are expected to occur on the nanosecond (or faster) time regime. Combining the lifetime information with that from single-particle spectroscopy (specifically the surface groups), may best relate the trends of the observed emission and surface groups.

Without such advanced techniques, work on the determination of Si NP reduction potentials with the addition of analytes may prove useful in understanding $\mathrm{Si}$ emission, potentially allowing $\mathrm{Si}$ NPs to replace CdSe QDs as single-QD nanosensors. In the meantime, if a charge transfer reaction is occurring between CdSe/ZnS QDs and 5-HT, then the oxidization products of 5-HT must still be identified. Though they have been difficult to isolate using standard NMR, FT-IR and UV spectroscopies, electron paramagnetic resonance and transient absorption spectroscopies should allow for the identification of short lived, 5-HT intermediates.

Addressing some of these described issues would not only expand the potential biological applications of Si, but also of NPs in general. There is a lot of work to be done before realizing the application of Si NPs as practical biological fluorophores, but the future looks bright! 


\section{Terminal References}

[1] G. Schmid, Nanoparticles: From Theory to Application, 1 ed., Wiley-VCH, 2004.

[2] A. P. Alivisatos, J. Phys. Chem. 1996, 100, 13226-13239.

[3] a.) A. P. Alivisatos, Science 1996, 271, 933-937; b.) C. B. Murray, D. J. Norris, M. G. Bawendi, J. Am. Chem. Soc. 1993, 115, 8706-8715; c.) Z. H. Kang, Y. Liu, C. H. A. Tsang, D. D. D. Ma, X. Fan, N. B. Wong, S. T. Lee, Adv. Mater. 2009, 21, 661; d.) R. J. Ellingson, M. C. Beard, J. C. Johnson, P. R. Yu, O. I. Micic, A. J. Nozik, A. Shabaev, A. L. Efros, Nano Lett. 2005, 5, 865-871.

[4] Y. Kanemitsu, Phys. Rep. 1995, 263, 1.

[5] a.) C. Sifuentes, Y. O. Barmenkov, A. N. Starodumov, V. N. Filippov, A. A. Lipovskii, Opt. Eng. 2000, 39, 2182-2186; b.) O. V. Vassiltsova, Z. Y. Zhao, M. A. Petrukhina, M. A. Carpenter, Sensor Actuat B 2007, 123, 522-529; c.) J. B. Baxter, E. S. Aydil, Appl. Phys. Lett. 2005, 86, 053114; d.) J. K. T. Anders Schei, Halvard Tveit, Production of High Silicon Alloys, Tapir Forlag, 1998; e.) H. Zimmerman, Silicon Optoelectronic Integrated Circuits, Vol. 1, Springer, 2004.

[6] a.) S. Caroli, Element Speciation in Bioinorganic Chemistry, Vol. 1, WileyInterscience, 1996; b.) M. P. Stewart, J. M. Buriak, Adv. Mater. 2000, 12, 859869; c.) C. Kirchner, T. Liedl, S. Kudera, T. Pellegrino, A. M. Javier, H. E. Gaub, S. Stolzle, N. Fertig, W. J. Parak, Nano Lett. 2005, 5, 331-338; d.) J. H. Park, L. Gu, G. von Maltzahn, E. Ruoslahti, S. N. Bhatia, M. J. Sailor, Nat. Mater. 2009, 8, 331-336; e.) W. J. Parak, D. Gerion, D. Zanchet, A. S. Woerz, T. Pellegrino, C. Micheel, S. C. Williams, M. Seitz, R. E. Bruehl, Z. Bryant, C. Bustamante, C. R. Bertozzi, A. P. Alivisatos, Chem. Mater. 2002, 14, 2113-2119; f.) L. Wang, V. Reipa, J. Blasic, Bioconjugate Chem. 2004, 15, 409-412.

[7] J. M. Klostranec, W. C. W. Chan, Adv. Mater. 2006, 18, 1953-1964.

[8] a.) A. Sukhanova, M. Devy, L. Venteo, H. Kaplan, M. Artemyev, V. Oleinikov, D. Klinov, M. Pluot, J. H. M. Cohen, I. Nabiev, Anal. Biochem. 2004, 324, 60-67; b.) U. Resch-Genger, M. Grabolle, S. Cavaliere-Jaricot, R. Nitschke, T. Nann, Nat. Methods 2008, 5, 763-775. 
[9] a.) Y. He, Y. Y. Su, X. B. Yang, Z. H. Kang, T. T. Xu, R. Q. Zhang, C. H. Fan, S. T. Lee, J. Am. Chem. Soc. 2009, 131, 4434-4438; b.) J. N. Mason, H. Farmer, I. D. Tomlinson, J. W. Schwartz, V. Savchenko, L. J. DeFelice, S. J. Rosenthal, R. D. Blakely, J. Neurosci. Methods 2005, 143, 3-25.

[10] a.) L. Manna, D. J. Milliron, A. Meisel, E. C. Scher, A. P. Alivisatos, Nat. Mater. 2003, 2, 382-385; b.) S. Pokrant, K. B. Whaley, Eur. Phys. J. D 1999, 6, 255-267;

c.) S. Kim, B. Fisher, H. J. Eisler, M. Bawendi, J. Am. Chem. Soc. 2003, 125, 11466-11467.

[11] S. Perkowitz, Optical Characterization of Semiconductors, Academic Press Limited, London, 1993.

[12] L. E. Brus, J. Chem. Phys. 1984, 80, 4403-4409.

[13] B. D. Fahlman, Materials Chemistry, Springer, 2007.

[14] M. Howarth, W. Liu, S. Puthenveetil, Y. Zheng, L. F. Marshall, M. M. Schmidt, K. D. Wittrup, M. G. Bawendi, A. Y. Ting, Nat. Methods 2008, 5, 397-399.

[15] M. A. Reed, J. N. Randall, R. J. Aggarwal, R. J. Matyi, T. M. Moore, A. E. Wetsel, Phys. Rev. Lett. 1988, 60, 535-537.

[16] a.) V. I. Klimov, Semiconductor and Metal Nanocrystals: Synthesis and Electronic and Optical Properties, Vol. 1, CC Press;, 2003; b.) N. F. Borrelli, D. W. Hall, H. J. Holland, D. W. Smith, J. Appl. Phys. 1987, 61, 5399-5409; c.) N. Chestnoy, R. Hull, L. E. Brus, J. Chem. Phys. 1986, 85, 2237-2242; d.) N. Herron, Y. Wang, H. Eckert, J. Am. Chem. Soc. 1990, 112, 1322-1326; e.) M. Bruchez, M. Moronne, P. Gin, S. Weiss, A. P. Alivisatos, Science 1998, 281, 2013-2016; f.) R. Rossetti, R. Hull, J. M. Gibson, L. E. Brus, J. Chem. Phys. 1985, 82, 552-559.

[17] J. M. Mauro, G. P. Anderson, E. R. Goldman, H. Mattoussi, B. L. Justus, NRL Review, 2002.

[18] a.) L. T. Canham, Appl. Phys. Lett. 1990, 57, 1046-1048; b.) D. J. Lockwood, J. Mater. Sci. 2009, 20, 235-244.

[19] C. M. Hessel, E. J. Henderson, J. G. C. Veinot, Chem. Mater. 2006, 18, 61396146. 
[20] a.) E. V. Rogozhina, D. A. Eckhoff, E. Gratton, P. V. Braun, J. Mater. Chem. 2006, 16, 1421-1430; b.) M. Rosso-Vasic, E. Spruijt, B. van Lagen, L. De Cola, H. Zuilhof, Small 2008, 4, 1835-1841; c.) J. H. Warner, A. Hoshino, K. Yamamoto, R. D. Tilley, Angew. Chem. Int. Ed. 2005, 44, 4550-4554; d.) X. M. Zhang, D. Neiner, S. Z. Wang, A. Y. Louie, S. M. Kauzlarich, Nanotechnology 2007, 18, 095601.

[21] a.) L. Ruizendaal, S. P. Pujari, V. Gevaerts, J. M. J. Paulusse, H. Zuilhof, Chem. Asian J. 2011, 6, 2776; b.) R. K. Baldwin, K. A. Pettigrew, J. C. Garno, P. P. Power, G. Y. Liu, S. M. Kauzlarich, J. Am. Chem. Soc. 2002, 124, 1150-1151.

[22] a.) C. M. Hessel, E. J. Henderson, J. A. Kelly, R. G. Cavell, T. K. Sham, J. G. C. Veinot, J. Phys. Chem. C 2008, 112, 14247-14254; b.) R. A. Bley, S. M. Kauzlarich, J. E. Davis, H. W. H. Lee, Chem. Mater. 1996, 8, 1881-1888; c.) H. Tamura, M. Ruckschloss, T. Wirschem, S. Veprek, Appl. Phys. Lett. 1994, 65, 1537-1539.

[23] R. D. Tilley, J. H. Warner, K. Yamamoto, I. Matsui, H. Fujimori, Chem. Commun. 2005, 1833-1835.

[24] J. P. Wilcoxon, G. A. Samara, P. N. Provencio, Phys. Rev. B 1999, 60, $2704-$ 2714.

[25] a.) B. A. Manhat, A. L. Brown, L. A. Black, J. B. A. Ross, K. Fichter, T. Vu, E. Richman, A. M. Goforth, Chem. Mater. 2011, 23, 2407-2418; b.) A. Shiohara, S. Hanada, S. Prabakar, K. Fujioka, T. H. Lim, K. Yamamoto, P. T. Northcote, R. D. Tilley, J. Am. Chem. Soc. 2010, 132, 248-253; c.) S. W. Lin, D. H. Chen, Small 2009, 5, 72-76.

[26] E. Rosenthal, K. R. Zinn, Optical Imaging of Cancer, Vol. 1, Springer, 2010.

[27] a.) J. Zhang, R. E. Campbell, A. Y. Ting, R. Y. Tsien, Nat. Rev. Mol. Cell Bio. 2002, 3, 906-918; b.) R. C. Narin, Fluorescent Protein Tracing, Churchill Livingstone, 1976.

[28] a.) E. Terpetschnig, H. Szmacinski, H. Malak, J. R. Lakowicz, Biophys. J. 1995, 68, 342-350; b.) V. Balzani, A. Juris, M. Venturi, S. Campagna, S. Serroni, Chem. Rev. 1996, 96, 759-833. 
[29] a.) W. D. Horrocks, D. R. Sudnick, Acc. Chem. Res. 1981, 14, 384-392; b.) I. Hemmila, V. Laitala, J. Fluoresc. 2005, 15, 529-542.

[30] a.) O. Ehlert, R. Thomann, M. Darbandi, T. Nann, ACS Nano 2008, 2, 120-124;

b.) X. H. Gao, S. R. Dave, in Bio-Applications of Nanoparticles, Vol. 620, Springer-Verlag Berlin, Berlin, 2007, pp. 57-73; c.) Z. F. Li, E. Ruckenstein, Nano Lett. 2004, 4, 1463-1467.

[31] a.) J. C. Hebden, S. R. Arridge, D. T. Delpy, Phys. Med. Biol. 1997, 42, 825-840;

b.) S. R. Arridge, J. C. Hebden, Phys. Med. Biol. 1997, 42, 841-853.

[32] W. T. Mason, Fluorescent and Luminescent Probes for Biological Activity: A Practical Guide to Technology for Quantitative Real-Time Analysis, Vol. 2, Avademic Press, 1999.

[33] Z. Cheng, Y. Wu, Z. M. Xiong, S. S. Gambhir, X. Y. Chen, Bioconjugate Chem. 2005, 16, 1433-1441.

[34] K. M. Fukuzawa, H., J. Phys. Chem. B 1968, 72, 371-372.

[35] W. H. Melhuish, J. Phys. Chem. B 1961, 65, 299-235.

[36] N. P. Geacintov, M.; Vogel, F., Phys. Rev. Lett. 1969, 22, 593-596.

[37] R. Katoh, K. Suzuki, A. Furube, M. Kotani, K. Tokumaru, J. Phys. Chem. C 2009, 113, 2961-2965.

[38] a.) C. C. L. Leznoff, A.B.P., 1986-1993, Vol 1-4; b.) N. B. Mckeown, Phthalocyanine Materials: Synthesis, Structure and Function (Chemistry of Solid State Materials), Vol. 1, Cambridge University Press, 1998.

[39] B. M. Krasovitskii, Organic Luminescent Materials, Vol. 1, Wiley-VCH, 1988.

[40] W. W. Yu, L. H. Qu, W. Z. Guo, X. G. Peng, Chem. Mater. 2003, 15, 2854-2860.

[41] A. Waggoner, Curr. Opin. Chem. Biol. 2006, 10, 62-66.

[42] a.) I. Chen, M. Howarth, W. Y. Lin, A. Y. Ting, Nat. Methods 2005, 2, 99-104;

b.) A. Miyawaki, A. Sawano, T. Kogure, Nat. Rev. Mol. Cell Bio. 2003, S1-S7.

[43] N. C. Institute, in http://www.cancer.gov/.

[44] a.) Y. Xing, Q. Chaudry, C. Shen, K. Y. Kong, H. E. Zhau, L. Wchung, J. A. Petros, R. M. O'Regan, M. V. Yezhelyev, J. W. Simons, M. D. Wang, S. Nie, Nat. Protocols 2007, 2, 1152-1165; b.) I. L. Medintz, H. T. Uyeda, E. R. Goldman, H. 
Mattoussi, Nat. Mater. 2005, 4, 435-446; c.) J. Choi, N. S. Wang, V. Reipa, Bioconjugate Chem. 2008, 19, 680-685; d.) J. Antelman, C. Wilking-Chang, S. Weiss, X. Michalet, Nano Lett. 2009, 9, 2199-2205; e.) C. Weber, S. Reiss, K. Langer, Int. J. Pharm. 2000, 211.

[45] a.) S. J. Clarke, C. A. Hollmann, Z. J. Zhang, D. Suffern, S. E. Bradforth, N. M. Dimitrijevic, W. G. Minarik, J. L. Nadeau, Nat. Mater. 2006, 5, 409-417; b.) I. L. Medintz, M. H. Stewart, S. A. Trammell, K. Susumu, J. B. Delehanty, B. C. Mei, J. S. Melinger, J. B. Blanco-Canosa, P. E. Dawson, H. Mattoussi, Nat. Mater. 2010, 9, 676-684.

[46] a.) S. R. Mujumdar, R. B. Mujumdar, C. M. Grant, A. S. Waggoner, Bioconjugate Chem. 1996, 7, 356-362; b.) V. Buschmann, K. D. Weston, M. Sauer, Bioconjugate Chem. 2003, 14, 195-204; c.) J. E. Berlier, A. Rothe, G. Buller, J. Bradford, D. R. Gray, B. J. Filanoski, W. G. Telford, S. Yue, J. X. Liu, C. Y. Cheung, W. Chang, J. D. Hirsch, J. M. Beechem, R. P. Haugland, J. Histochem. Cytochem. 2003, 51, 1699-1712.

[47] a.) H. C. Lu, G. S. Yi, S. Y. Zhao, D. P. Chen, L. H. Guo, J. Cheng, J. Mater. Chem. 2004, 14, 1336-1341; b.) N. Panchuk-Voloshina, R. P. Haugland, J. Bishop-Stewart, M. K. Bhalgat, P. J. Millard, F. Mao, W. Y. Leung, J. Histochem. Cytochem. 1999, 47, 1179-1188; c.) C. Eggeling, A. Volkmer, C. A. M. Seidel, ChemPhysChem 2005, 6, 791-804.

[48] a.) I. G. Roberts, P. Fallon, F. J. Kirkham, P. M. Kirshbom, C. E. Cooper, M. J. Elliott, A. D. Edwards, J. Thorac. Cardiovasc, Surg, 1998, 115, 94; b.) I. Miyashiro, Open Surg. Onco.l J. 2010, 2.

[49] Y. He, Z. H. Kang, Q. S. Li, C. H. A. Tsang, C. H. Fan, S. T. Lee, Angew. Chem. Int. Ed. 2009, 48, 128-132.

[50] D. Gerion, F. Pinaud, S. C. Williams, W. J. Parak, D. Zanchet, S. Weiss, A. P. Alivisatos, J. Phys. Chem. B 2001, 105, 8861-8871.

[51] a.) J. L. Zhao, J. A. Bardecker, A. M. Munro, M. S. Liu, Y. H. Niu, I. K. Ding, J. D. Luo, B. Q. Chen, A. K. Y. Jen, D. S. Ginger, Nano Lett. 2006, 6, 463-467; b.) V. L. Colvin, M. C. Schlamp, A. P. Alivisatos, Nature 1994, 370, 354-357. 
[52] a.) M. Law, L. E. Greene, J. C. Johnson, R. Saykally, P. D. Yang, Nat. Mater. 2005, 4, 455-459; b.) I. Lokteva, N. Radychev, F. Witt, H. Borchert, J. Parisi, J. Kolny-Olesiak, J. Phys. Chem. C 2010, 114, 12784-12791; c.) R. Vogel, K. Pohl, H. Weller, Chem. Phys. Lett. 1990, 174, 241-246.

[53] G. Konstantatos, E. H. Sargent, P. IEEE 2009, 97, 1666.

[54] a.) R. C. Somers, M. G. Bawendi, D. G. Nocera, Chem. Soc. Rev. 2007, 36, 579591; b.) M. A. Walling, J. A. Novak, J. R. E. Shepard, Int. J. Mol. Sci. 2009, 10, 441-491; c.) C. J. Murphy, Anal, Chem. 2002, 74, 520A-526A; d.) I. L. Medintz, H. Mattoussi, Phys. Chem. Chem. Phys. 2009, 11, 17-45; e.) S. Mazumder, R. Dey, M. K. Mitra, S. Mukherjee, G. C. Das, J. Nanomaterials 2009, 815734.

[55] a.) Y. H. Sun, Y. S. Liu, P. T. Vernier, C. H. Liang, S. Y. Chong, L. Marcu, M. A. Gundersen, Nanotechnology 2006, 17, 4469-4476; b.) J. K. Jaiswal, H. Mattoussi, J. M. Mauro, S. M. Simon, Nat. Biotechnol. 2003, 21, 47-51.

[56] J. M. Costa-Fernandez, R. Pereiro, A. Sanz-Medel, Trac-Trends Anal. Chem. 2006, 25, 207-218.

[57] a.) A. H. Fu, W. W. Gu, B. Boussert, K. Koski, D. Gerion, L. Manna, M. Le Gros, C. A. Larabell, A. P. Alivisatos, Nano Lett. 2007, 7, 179-182; b.) J. K. Jaiswal, E. R. Goldman, H. Mattoussi, S. M. Simon, Nat. Methods 2004, 1, 73-78; c.) V. Jacquier, M. Prummer, J. M. Segura, H. Pick, H. Vogel, Proc. Natl. Acad. Sci. U. S. A. 2006, 103, 14325-14330.

[58] a.) Y. P. Chang, F. Pinaud, J. Antelman, S. Weiss, J. Biophotonics 2008, 1, 287298; b.) L. Ferreira, J. M. Karp, L. Nobre, R. Langer, Cell Stem Cell 2008, 3, 136146.

[59] a.) N. Singh, R. C. Mulrooney, N. Kaur, J. F. Callan, J. Fluoresc. 2009, 19, 777 782; b.) Y. F. Chen, Z. Rosenzweig, Anal, Chem. 2002, 74, 5132-5138; c.) Y.-h. Zhang, H.-s. Zhang, X.-f. Guo, H. Wang, Microchemical Journal 2008, 89, 142147; d.) N. Singh, R. C. Mulrooney, N. Kaur, J. F. Callan, Chem. Commun. 2008, 4900-4902.

[60] a.) X. Q. Yan, Z. B. Shang, Z. Zhang, Y. Wang, W. J. Jin, Luminescence 2009, 24, 255-259; b.) D. B. Cordes, S. Gamsey, B. Singaram, Angew. Chem. Int. Ed. 
2006, 45, 3829-3832; c.) C.-P. Huang, S.-W. Liu, T.-M. Chen, Y.-K. Li, Sensor Actuat B 2008, 130, 338-342; d.) I. L. Medintz, E. R. Goldman, M. E. Lassman, J. M. Mauro, Bioconjugate Chem. 2003, 14, 909-918; e.) I. L. Medintz, A. R. Clapp, H. Mattoussi, E. R. Goldman, B. Fisher, J. M. Mauro, Nat. Mater. 2003, 2, 630638.

[61] a.) X. Wang, J. Wu, F. Li, H. Li, Nanotechnology 2008, 19, 205501; b.) C. Han, H. Li, Small 2008, 4, 1344-1350; c.) S. Huang, Q. Xiao, R. Li, H.-L. Guan, J. Liu, X.-R. Liu, Z.-K. He, Y. Liu, Anal. Chim. Acta. 2009, 645, 73-78.

[62] a.) S. J. Rosenthal, A. Tomlinson, E. M. Adkins, S. Schroeter, S. Adams, L. Swafford, J. McBride, Y. Q. Wang, L. J. DeFelice, R. D. Blakely, J. Am. Chem. Soc. 2002, 124, 4586-4594; b.) C. P. Soman, T. D. Giorgio, Langmuir 2008, 24 , 4399-4404.

[63] a.) M. Suzuki, Y. Husimi, H. Komatsu, K. Suzuki, K. T. Douglas, J. Am. Chem. Soc. 2008, 130, 5720-5725; b.) C. Y. Zhang, J. Hu, Anal, Chem. 2010, 82, 19211927.

[64] a.) X. Liu, L. Cheng, J. Lei, H. Ju, Analyst 2008, 133, 1161-1163; b.) D. R. Cooper, D. Suffern, L. Carlini, S. J. Clarke, R. Parbhoo, S. E. Bradforth, J. L. Nadeau, Phys. Chem. Chem. Phys. 2009, 11, 4298-4310.

[65] T. Pons, I. L. Medintz, X. Wang, D. S. English, H. Mattoussi, J. Am. Chem. Soc. 2006, 128, 15324-15331.

[66] a.) J. A. J. Schmitt, M. Wingen, J. G. Ramaekers, E. A. T. Evers, W. J. Riedel, Curr. Pharm. Des. 2006, 12, 2473-2486; b.) A. V. Terry, J. J. Buccafusco, C. Wilson, Behav. Brain Res. 2008, 195, 30-38.

[67] a.) I. Lucki, Bio. Psych. 1998, 44, 151-162; b.) C. P. Muller, R. J. Carey, J. P. Huston, M. A. D. Silva, Prog. Neurobiol. 2007, 81, 133-178; c.) A. L. LopezFigueroa, C. S. Norton, M. O. Lopez-Figueroa, D. Armellini-Dodel, S. Burke, H. Akil, J. F. Lopez, S. J. Watson, Bio. Psych. 2004, 55, 225-233.

[68] P. T. Snee, Y. H. Chan, D. G. Nocera, M. G. Bawendi, Adv. Mater. 2005, 17, 1131. 
[69] B. O. Dabbousi, J. RodriguezViejo, F. V. Mikulec, J. R. Heine, H. Mattoussi, R. Ober, K. F. Jensen, M. G. Bawendi, J. Phys. Chem. B 1997, 101, 9463-9475.

[70] a.) M. C. Mancini, B. A. Kairdolf, A. M. Smith, S. M. Nie, J. Am. Chem. Soc. 2008, 130, 10836; b.) K. Pechstedt, T. Whittle, J. Baumberg, T. Melvin, J. Phys. Chem. C 2010, 114, 12069-12077.

[71] a.) S. R. Cordero, P. J. Carson, R. A. Estabrook, G. F. Strouse, S. K. Buratto, J. Phys. Chem. B 2000, 104, 12137-12142; b.) Y. Zhang, J. He, P. N. Wang, J. Y. Chen, Z. J. Lu, D. R. Lu, J. Guo, C. C. Wang, W. L. Yang, J. Am. Chem. Soc. 2006, 128, 13396-13401; c.) A. Y. Nazzal, L. H. Qu, X. G. Peng, M. Xiao, Nano Lett. 2003, 3, 819-822.

[72] J. R. Lakowicz, Principles of Fluorescence Spectroscopy, Plenum Press, New York, 1983.

[73] I. N. Germanenko, S. T. Li, M. S. El-Shall, J. Phys. Chem. B 2001, 105, 59-66.

[74] a.) R. L. Bowman, P. A. Caulfield, S. Udenfriend, Science 1955, 122, 32-33; b.) R. Chen, Biochemistry 1968, 60, 598; c.) J. B. S. Nag, P.K. Madhu, S. Maiti, Biophys. J. 2008, 94, 4145.

[75] S. N. Sharma, Z. S. Pillai, P. V. Kamat, J. Phys. Chem. B 2003, 107, 1008810093.

[76] a.) D. Neuman, A. D. Ostrowski, A. A. Mikhailovsky, R. O. Absalonson, G. F. Strouse, P. C. Ford, J. Am. Chem. Soc. 2008, 130, 168-175; b.) S. Sadhu, A. Patra, ChemPhysChem 2008, 9, 2052-2058.

[77] M. R. Eftink, L. A. Selvidge, Biochemistry 1982, 21, 117-125.

[78] a.) A. M. Gardier, D. J. David, G. Jego, C. Przybylski, C. Jacquot, S. Durier, B. Gruwez, E. Douvier, P. Beauverie, N. Poisson, R. Hen, M. Bourin, J. Neurosci. 2003, 86, 13; b.) N. Bel, F. Artigas, Eur. J. Pharmacol. 1992, 229, 101-103; c.) R. Tao, Z. Y. Ma, S. B. Auerbach, J. Pharmacol. Exp. The, 2000, 294, 571-579.

[79] a.) P. Svenningsson, K. Chergui, I. Rachleff, M. Flajolet, X. Q. Zhang, M. El Yacoubi, J. M. Vaugeois, G. G. Nomikos, P. Greengard, Science 2006, 311, 7780; b.) Y. Sari, Neurosci. Biobehav. Rev. 2004, 28, 565-582; c.) M. S. Clark, J. F. Neumaier, 2002, 35, 170-185. 
[80] K. M. Fichter, M. Flajolet, P. Greengard, T. Q. Vu, Proc. Natl. Acad. Sci. U. S. A. 2010, 107, 18658-18663.

[81] C. A. Blanco-Centurion, R. J. Salin-Pascual, Brain Res. 2001, 923, 128-136.

[82] X. Ji, G. Palui, T. Avellini, H. B. Na, C. Yi, K. L. Knappenberger, Jr., H. Mattoussi, J. Am. Chem. Soc. 2012, 134, 6006-6017.

[83] S. M. Liu, J. Nanosci. Nanotechnol. 2008, 8, 1110-1125.

[84] a.) M. Green, E. Howman, Chem. Commun. 2005, 121-123; b.) D. R. Cooper, N. M. Dimitrijevic, J. L. Nadeau, Nanoscale 2010, 2, 114-121.

[85] B. I. Ipe, M. Lehnig, C. M. Niemeyer, Small 2005, 1, 706-709.

[86] a.) M. Samadpour, P. P. Boix, S. Gimenez, A. I. Zad, N. Taghavinia, I. MoraSero, J. Bisquert, J. Phys. Chem. C 2011, 115, 14400-14407; b.) K. Prabakar, S. Minkyu, S. Inyoung, K. Heeje, J, Phys. D 2010, 43, 012002; c.) T. Shu, Z. Zhou, H. Wang, G. Liu, P. Xiang, Y. Rong, H. Han, Y. Zhao, J. Mater. Chem. 2012, 22, 10525-10529.

[87] a.) J. Jasieniak, J. Pacifico, R. Signorini, A. Chiasera, M. Ferrari, A. Martucci, P. Mulvaney, Adv. Funct. Mater. 2007, 17, 1654-1662; b.) I. Robel, M. Kuno, P. V. Kamat, J. Am. Chem. Soc. 2007, 129, 4136.

[88] D. L. Robinson, B. J. Venton, M. Heien, R. M. Wightman, Clin. Chem. 2003, 49, 1763-1773.

[89] M. Z. Wrona, G. Dryhurst, Chem. Res. Tox. 1998, 11, 639-650.

[90] a.) Y. Kanemitsu, Phys. Rev. B 1994, 49, 16845-16848; b.) G. G. Qin, H. Z. Song, B. R. Zhang, J. Lin, J. Q. Duan, G. Q. Yao, Phys. Rev. B 1996, 54, 2548-2555.

[91] S. Godefroo, M. Hayne, M. Jivanescu, A. Stesmans, M. Zacharias, O. I. Lebedev, G. Van Tendeloo, V. V. Moshchalkov, Nat.Nanotechnology 2008, 3, 174-178.

[92] a.) J. P. Wilcoxon, G. A. Samara, Appl. Phys. Lett. 1999, 74, 3164-3166; b.) Z. H. Kong, Y. Liu, C. H. A. Tsang, D. D. D. Ma, X. Fan, N. B. Wong, S. T. Lee, $A d v$. Mater. 2009, 21, 661 .

[93] a.) L. Ferraioli, M. Wang, G. Pucker, D. Navarro-Urrios, N. Daldosso, C. Kompocholis, L. Pavesi, J. Nanomaterials 2007; b.) A. Gupta, M. T. Swihart, H. Wiggers, Adv. Funct. Mater. 2009, 19, 696-703; c.) J. D. Holmes, K. J. Ziegler, R. 
C. Doty, L. E. Pell, K. P. Johnston, B. A. Korgel, J. Am. Chem. Soc. 2001, 123, 3743-3748; d.) J. G. C. Veinot, Chem. Commun. 2006, 4160-4168.

[94] D. Neiner, H. W. Chiu, S. M. Kauzlarich, J. Am. Chem. Soc. 2006, 128, 1101611017.

[95] a.) J. Nelles, D. Sendor, A. Ebbers, F. M. Petrat, H. Wiggers, C. Schulz, U. Simon, Colloid. Polym. Sci. 2007, 285, 729-736; b.) J. M. Buriak, Chem. Rev. 2002, 102, 1271-1308.

[96] M. Rosso-Vasic, E. Spruijt, Z. Popovic, K. Overgaag, B. van Lagen, B. Grandidier, D. Vanmaekelbergh, D. Dominguez-Gutierrez, L. De Cola, H. Zuilhof, J. Mater. Chem. 2009, 19, 5926-5933.

[97] a.) G. Belomoin, J. Therrien, M. Nayfeh, Appl. Phys. Lett. 2000, 77, 779-781; b.) Z. Y. Zhou, L. Brus, R. Friesner, Nano Lett. 2003, 3, 163-167; c.) J. M. Rehm, G. L. McLendon, L. Tsybeskov, P. M. Fauchet, Appl. Phys. Lett. 1995, 66, 3669; d.) S. K. Chiu, B. A. Manhat, W. J. I. DeBenedetti, A. L. Brown, K. Fichter, T. Vu, M. Eastman, J. Jiao, A. M. Goforth, J. Mater. Res. 2012; e.) W. J. I. DeBenedetti, B. A. Manhat, D. J. Tweet, A. M. Goforth, 2012.

[98] a.) K. A. Pettigrew, Q. Liu, P. P. Power, S. M. Kauzlarich, Chem. Mater. 2003, 15, 4005-4011; b.) D. S. English, L. E. Pell, Z. H. Yu, P. F. Barbara, B. A. Korgel, Nano Lett. 2002, 2, 681-685.

[99] I. N. Germanenko, M. Dongol, Y. B. Pithawalla, M. S. El-Shall, J. A. Carlisle, Pure Appl. Chem. 2000, 72, 245-255.

[100] K. B. Sundaram, J. Alizadeh, Thin Solid Films 2000, 370, 151.

[101] S. Sato, M. T. Swihart, Chem. Mater. 2006, 18, 4083-4088.

[102] R. Plass, S. Pelet, J. Krueger, M. Gratzel, U. Bach, J. Phys. Chem. B 2002, 106, 7578-7580.

[103] X. H. Gao, Y. Y. Cui, R. M. Levenson, L. W. K. Chung, S. M. Nie, Nat. Biotechnol. 2004, 22, 969-976.

[104] M. Kondoh, S. Araragi, K. Sato, M. Higashimoto, M. Takiguchi, M. Sato, Toxicology 2002, 170, 111-117.

[105] D. E. Morse, Trends Biotechnol. 1999, 17, 230-232. 
[106] D. Jurbergs, E. Rogojina, L. Mangolini, U. Kortshagen, Appl. Phys. Lett. 2006, $88, \mathrm{p} 233116$.

[107] a.) K. Kravitz, A. Kamyshny, A. Gedanken, S. Magdassi, J.Solid State Chem. 2010, 183, 1442-1447; b.) N. A. Dhas, C. P. Raj, A. Gedanken, Chem. Mater. 1998, 10, 3278; c.) Z. H. Kang, C. H. A. Tsang, N. B. Wong, Z. D. Zhang, S. T. Lee, Journal of the American Chemical Society 2007, 129, 12090-+; d.) P. Shen, N. Uesawa, S. Inasawa, Y. Yamaguchi, J. Mater. Chem. 2010, 20, 1669-1675; e.) C. J. Powell, A. Jablonski, J. Phy. Chem. Ref. Data 1999, 28, 19-62.

[108] N. Shirahata, M. R. Linford, S. Furumi, L. Pei, Y. Sakka, R. J. Gates, M. C. Asplund, Chem. Commun. 2009, 4684-4686.

[109] a.) A. S. Heintz, M. J. Fink, B. S. Mitchell, Adv. Mater. 2007, 19, 3984; b.) A. S. Heintz, M. J. Fink, B. S. Mitchell, Appl. Organomet. Chem. 2010, 24, 236-240.

[110] J. H. Warner, H. Rubinsztein-Dunlop, R. D. Tilley, J. Phys. Chem. B 2005, 109, 19064-19067.

[111] Q. Liu, S. M. Kauzlarich, Mat. Sci. Eng. B 2002, 96, 72-75.

[112] H. J. Yvon, A Guide to Recording Fluorescence Quantum Yields.

[113] M. G. Badea, L. Brand, Meth. Enzym. 1974, 61, 378-425.

[114] A. Grinvald, Steinber.Iz, Anal. Biochem. 1974, 59, 583-598.

[115] a.) D. Neiner, S. M. Kauzlarich, Chem. Mater. 2010, 22, 487-493; b.) P. F. McMillan, J. Gryko, C. Bull, R. Arledge, A. J. Kenyon, B. A. Cressey, J. Solid State Chem. 2005, 178, 937-949.

[116] M. Ray, K. Jana, N. R. Bandyopadhyay, S. M. Hossain, D. Navarro-Urrios, P. P. Chattyopadhyay, M. A. Green, Solid State Commun. 2009, 149, 352-356.

[117] A. T. Heitsch, C. M. Hessel, V. A. Akhavan, B. A. Korgel, Nano Lett. 2009, 9, 3042-3047.

[118] C. D. N. Wagner, A.V.; Kraut-Vass, A. ; Allison, J.W.; Powell, C.J.; Rumble, Jr, J. R;, NIST X-ray Photoelectron Spectroscopy Database, Version 3.5 2003.

[119] L. B. Feng, Y. L. Wang, N. Wang, Y. X. Ma, Polymer Bull. 2009, 63, 313-327.

[120] M. A. Filler, J. A. Van Deventer, A. J. Keung, S. F. Bent, J. Am. Chem. Soc. 2006, 128, 770-779. 
[121] S. Kim, E. Kim, W. Kim, J. Colloid Interface Sci. 2005, 292, 93-98.

[122] H. J. Kim, Q. Shao, Y. H. Kim, Surf. Coat. Tech. 2003, 171, 39-45.

[123] J. Zou, P. Sanelle, K. A. Pettigrew, S. M. Kauzlarich, J. Cluster Sci. 2006, 17, 565-578.

[124] a.) K. Kusova, O. Cibulka, K. Dohnalova, I. Pelant, A. Fucikova, J. Valenta, Physica E 2009, 41, 982-985; b.) N. Shirahata, T. Hasegawa, Y. Sakka, T.

Tsuruoka, Small 2010, 6, 915-921.

[125] E. Rogozhina, G. Belomoin, A. Smith, L. Abuhassan, N. Barry, O. Akcakir, P. V. Braun, M. H. Nayfeh, Appl. Phys. Lett. 2001, 78, 3711-3713.

[126] V. Mahalingam, S. Onclin, M. Peter, B. J. Ravoo, J. Huskens, D. N. Reinhoudt, Langmuir 2004, 20, 11756-11762.

[127] a.) S. Yang, W. Li, B. Cao, H. Zeng, W. Cai, J. Phys. Chem. C 2011, 115, 2105621062; b.) P. D. J. Calcott, Mat. Sci. Eng. B 1998, 51, 132-140; c.) A. J. Kontkiewicz, A. M. Kontkiewicz, J. Siejka, S. Sen, G. Nowak, A. M. Hoff, P. Sakthivel, K. Ahmed, P. Mukherjee, S. Witanachchi, J. Lagowski, Appl. Phys. Lett. 1994, 65, 1436-1438.

[128] a.) A. N. Trukhin, M. Goldberg, J. Jansons, H. J. Fitting, I. A. Tale, J. Non-Cryst. Solids 1998, 223, 114-122; b.) A. Shluger, E. Stefanovich, Phys. Rev. B 1990, 42, 9664-9673.

[129] W. de Boer, D. Timmerman, K. Dohnalova, I. N. Yassievich, H. Zhang, W. J. Buma, T. Gregorkiewicz, Nat.Nanotechnology 2010, 5, 878-884.

[130] L. Brus, J. Phys. Chem. 1994, 98, 3575-3581.

[131] X. H. Gao, W. C. W. Chan, S. M. Nie, J. Biomed. Opt. 2002, 7, 532-537.

[132] J. M. Lauerhaas, G. M. Credo, J. L. Heinrich, M. J. Sailor, J. Am. Chem. Soc. 1992, 114, 1911-1912.

[133] J. K. M. Chun, A. B. Bocarsly, T. R. Cottrell, J. B. Benziger, J. C. Yee, J. Am. Chem. Soc. 1993, 115, 3025-3025.

[134] a.) B. SwerydaKrawiec, R. R. ChandlerHenderson, J. L. Coffer, Y. G. Rho, R. F. Pinizzotto, J. Phys. Chem. 1996, 100, 13776-13780; b.) R. R. Chandlerhenderson, B. Swerydakrawiec, J. L. Coffer, J. Phys. Chem. 1995, 99, 8851-8855. 
[135] M. C. Ko, G. J. Meyer, Chem. Mater. 1995, 7, 12-14.

[136] S. T. Li, I. N. Germanenko, M. S. El-Shall, J. Phys. Chem. B 1998, 102.

[137] D. Andsager, J. Hilliard, J. M. Hetrick, L. H. Abuhassan, M. Plisch, M. H. Nayfeh, J. Appl. Phys. 1993, 74.

[138] W. D. Kirkey, Y. Sahoo, X. G. Li, Y. Q. He, M. T. Swihart, A. N. Cartwright, S. Bruckenstein, P. N. Prasad, J. Mater. Chem. 2005, 15.

[139] H. Jang, L. E. Pell, B. A. Korgel, D. S. English, Journal of Photochemistry and Photobiology a-Chemistry 2003, 158.

[140] S. Yang, W. Cai, G. Liu, H. Zeng, P. Liu, J. Phys. Chem. C 2009, 113, 64806484.

[141] E. J. Henderson, J. A. Kelly, J. G. C. Veinot, Chem. Mater. 2009, 21.

[142] a.) M. M. Miller, A. A. Lazarides, J. Phys. Chem. B 2005, 109, 21556-21565; b.) K. S. Lee, M. A. El-Sayed, J. Phys. Chem. B 2006, 110, 19220-19225; c.) P. K. Jain, X. H. Huang, I. H. El-Sayed, M. A. El-Sayed, Acc. Chem. Res. 2008, 41, 1578-1586.

[143] V. Amendola, O. M. Bakr, F. Stellacci, Plasmonics 2010, 5, 85-97.

[144] a.) M. A. Green, J. H. Zhao, A. H. Wang, P. J. Reece, M. Gal, Nature 2001, 412, 805-808; b.) H. M. Mcconnell, J. C. Owicki, J. W. Parce, D. L. Miller, G. T. Baxter, H. G. Wada, S. Pitchford, Science 1992, 257, 1906-1912.

[145] G. Allan, C. Delerue, M. Lannoo, Phys. Rev. Lett. 1996, 76.

[146] G. G. Qin, Y. Q. Jia, Solid State Commun. 1993, 86, 559-563.

[147] a.) R. L. Cicero, M. R. Linford, C. E. D. Chidsey, Langmuir 2000, 16, 5688-5695; b.) R. Boukherroub, S. Morin, D. D. M. Wayner, F. Bensebaa, G. I. Sproule, J. M. Baribeau, D. J. Lockwood, Chem. Mater. 2001, 13, 2002-2011; c.) J. M. Buriak, Chem. Commun. 1999, 1051-1060; d.) A. B. Sieval, V. Vleeming, H. Zuilhof, E. J. R. Sudholter, Langmuir 1999, 15, 8288-8291; e.) A. Langner, A. Panarello, S. Rivillon, O. Vassylyev, J. G. Khinast, Y. J. Chabal, J. Am. Chem. Soc. 2005, 127, 12798-12799; f.) M. R. Linford, P. Fenter, P. M. Eisenberger, C. E. D. Chidsey, J. Am. Chem. Soc. 1995, 117, 3145-3155.

[148] I. H \& M Analytical Services, 2002. 


\section{Supporting Information for Introduction Chapters}

Calculate the number of atoms in a 1-nm Au nanoparticle:

radius $=\mathrm{r}=1 \mathrm{~nm}=1 \times 10^{-9} \mathrm{~m}=1 \times 10^{-7} \mathrm{~cm}$

volume $=\mathrm{V}=\frac{4}{3} \pi r^{3}=4.1888 \times 10^{-21} \mathrm{~cm}^{3}$

density $=\rho_{\mathrm{Au}}=19.3 \mathrm{~g} / \mathrm{cm}^{3}$

mass $=\mathrm{V} * \rho_{\mathrm{Au}}=8.0843 \times 10^{-20} \mathrm{gr}$

moles $=\frac{\text { mass }}{197 \mathrm{~g} / \mathrm{mol}}=4.103 \times 10^{-22}$ moles

number of atoms $=$ moles $*$ Avogadro's number $\left(6.022 \times 10^{23}\right)=247$ atoms

Solution to the Energy Values that satisfy the Schrödinger Equation (1D)

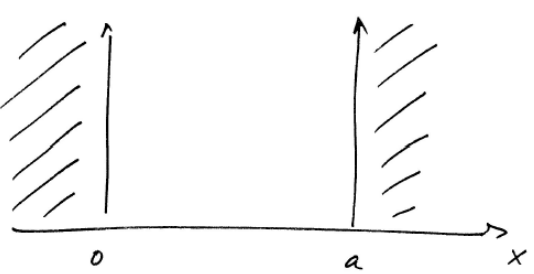

Figure A.1. Drawing of 1D infinite box

Time independent Schrödinger Equation $\quad \frac{-\hbar^{2}}{2 m} \frac{\partial^{2}}{d x^{2}} \Psi(x)=E \Psi(x)$

Rearrange the equation so the potential is zero, which occurs at the boundaries of the $1 \mathrm{D}$

box $\frac{\partial^{2}}{d x^{2}} \Psi(x)+k^{2} \Psi(x)=0, \mathrm{k}^{2}=\frac{2 m E}{\hbar^{2}}$ 
When differentiated twice, the general expression becomes $\Psi(x)=A e^{i k x}+B e^{-i k x}$

The real (or non-complex) solution becomes

$$
\Psi(x)=A \cos (k x)+B \sin (k x)
$$

When the boundary conditions are applied,

$\Psi(\mathrm{x})$ is continous (or equals 0 ) at $\mathrm{x}=0$ and $\mathrm{x}=\mathrm{a}$

$$
\begin{array}{ll}
\Psi(0)=0=A \cos (k 0)+B \sin (k 0 & \Psi(a)=0=0 \cos (k a)+B \sin (k a) \\
\Psi(0)=0=A(1)+B(0) & \Psi(a)=0=B \sin (k a), \text { which is only true when } k=\frac{n \pi}{L} \\
\Psi(0)=0 \text { only when } \mathrm{A}=0 & \Psi(a)=0=B \sin \left(\frac{n \pi}{L} a\right)
\end{array}
$$

After normalizing the wave function (to equal 1) and integrating:

$$
\begin{aligned}
& \int_{-\infty}^{\infty} \Psi^{*}(x) \Psi(x) d x=1 \\
& B^{2} \int_{-\infty}^{\infty} \sin 2\left(\frac{n \pi}{L} x\right) d x=1 \\
& B^{2} \int_{0}^{L} \sin 2\left(\frac{n \pi}{L} x\right) d x=\frac{x}{2}-\frac{L}{4 n \pi} \sin \left(\frac{2 n \pi}{L} x\right) \\
& \text { when evaluated at the limits }:=\frac{L}{2} \\
& B^{2} \frac{L}{2}=1 ; B=\sqrt{\frac{2}{L}} \\
& \Psi(x)=0=\sqrt{\frac{2}{L}} \sin \left(\frac{n \pi}{L} x\right)
\end{aligned}
$$

To calculate the energy value:

recall we defined :

$$
\begin{aligned}
& k=\frac{n \pi}{L} \& k=\sqrt{\frac{2 m E}{\hbar^{2}}} \therefore \frac{n \pi}{L}=\sqrt{\frac{2 m E}{\hbar^{2}}} \\
& E=\frac{n^{2} \pi^{2} \hbar^{2}}{2 m L^{2}}, \hbar=\frac{h}{2 \pi} \therefore E=\frac{n^{2} h^{2}}{8 m L^{2}}
\end{aligned}
$$


Table A1. Absorption and scattering coefficient for breast tissue and water.

\begin{tabular}{ccc}
\hline Material & Absorption $\left(\mu_{\mathrm{a}}\right)$ coefficient $\left(\mathrm{cm}^{-1}\right)$ & Scattering $\left(\mu_{\mathrm{s}}\right)$ coefficient $\left(\mathrm{cm}^{-1}\right)$ \\
\hline Breast tissue & $14.8(@ 460 \mathrm{~nm})$ & $213(@ 460 \mathrm{~nm})$ \\
Water & $.001($ all light $)$ & $.003($ all light $)$ \\
\hline
\end{tabular}




\section{Supporting Data and Radical Determination for CdSe/ZnS QDs as Serotonin}

\section{Nanosensors}
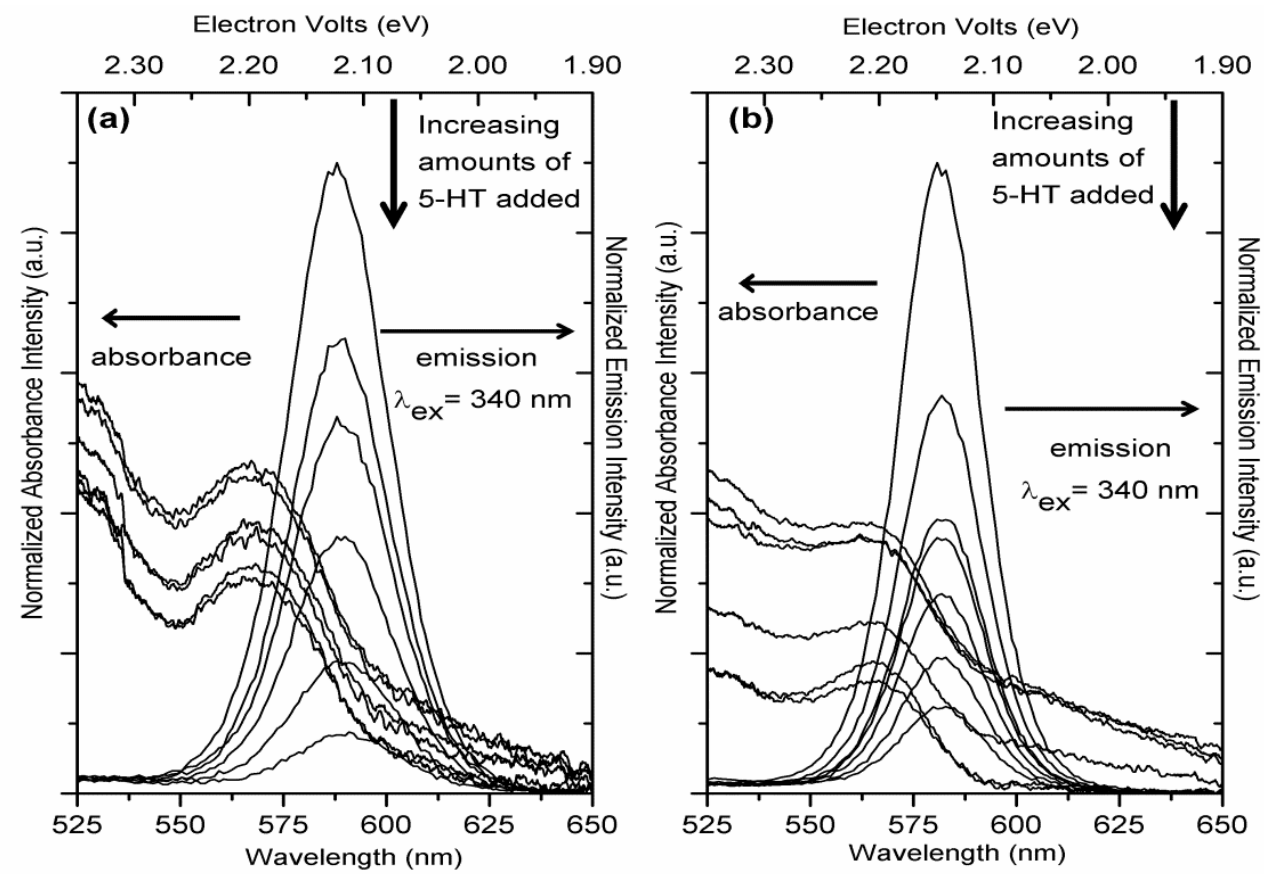

Figure B.1. Absorbance and emission spectra $\left(\lambda_{\mathrm{ex}}=340 \mathrm{~nm}\right)$ of (a) commercially available $585 \mathrm{~nm}$-emitting and (b) laboratory prepared $580 \mathrm{~nm}$-emitting CdSe@ZnS QDs. The emission $\lambda_{\max }$ of both batches of QDs was found to decrease in intensity when exposed to increasing amounts of 5-HT. 


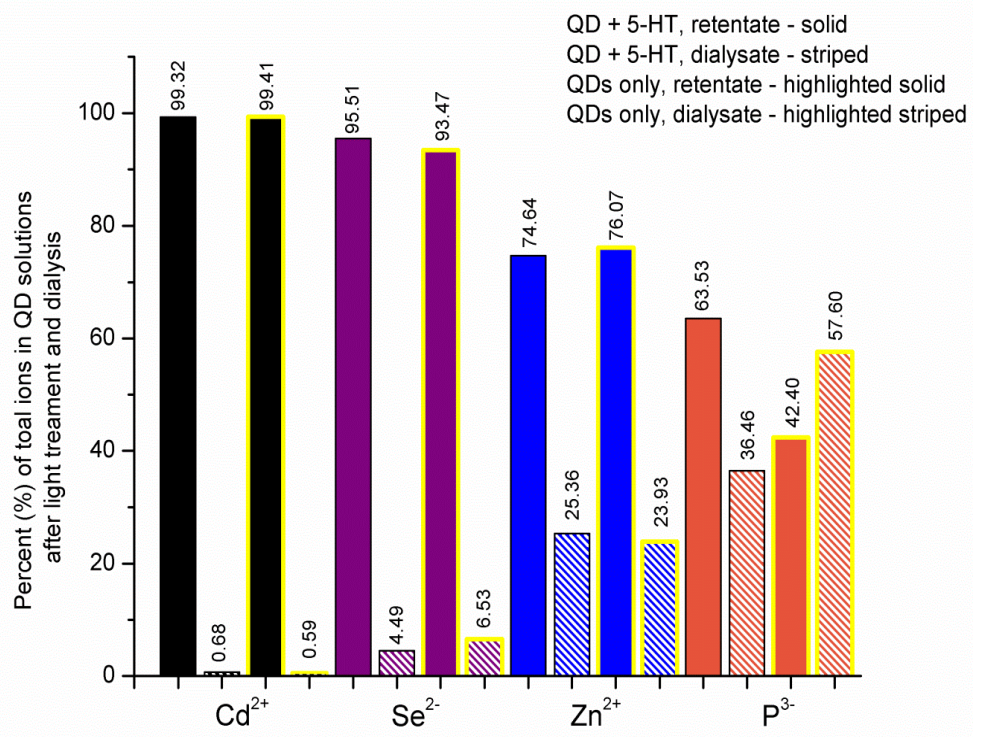

Figure B.2. Complete retentate (solid) and dialysate (checkered) of $\mathrm{Cd}^{2+}, \mathrm{Se}^{2-}, \mathrm{Zn}^{2+}$, and $\mathrm{P}^{3-}$ from ICP-MS analysis for the QDs + 5-HT and neat QDs only (highlighted) solutions after light treatment and dialysis. Note that $\mathrm{S}^{2-}$ cannot be discriminated via ICP-MS because its mass is the same as $\mathrm{O}_{2}$.
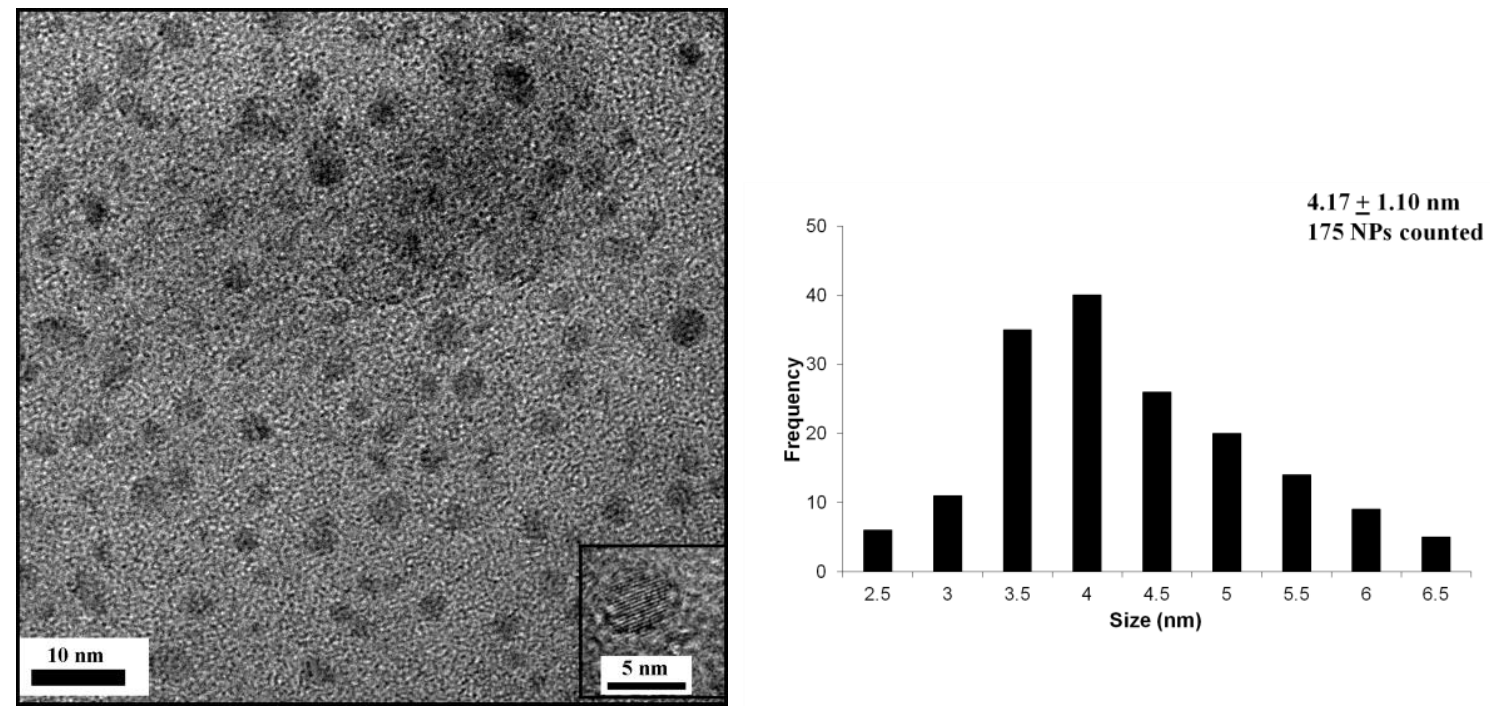

Figure B.3 Transmission Electron Microscopy images of CdSe/ZnS QDs. The average size was determined to be $4.16 \pm 1.10 \mathrm{~nm}$ after manually measuring over 175 particles. 


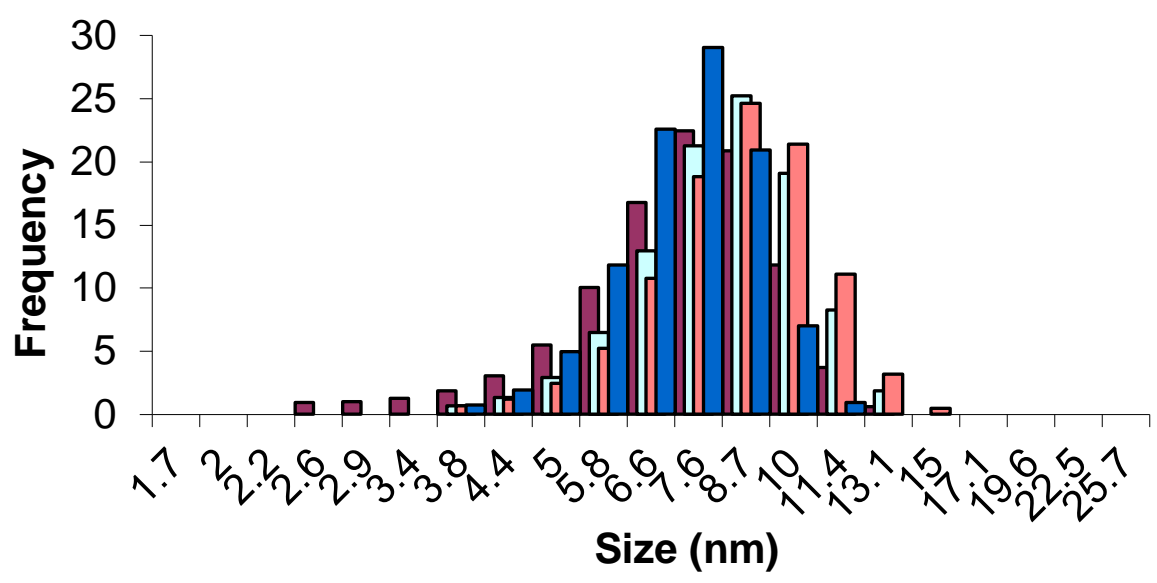

Figure B.4. DLS data of5 trials of CdSe QDs ${ }_{580}$. The effective hydrodynamic diameters of the QDs shell and stabilizing ligands is about $8.4 \mathrm{~nm}$

\section{Determination of the Involvement of Molecular Oxygen and oxygenated-centered Reactive Oxygen Species (ROS)}

To directly investigate the QD emission-dependence on oxygen, an experiment was conducted in which $10 \mathrm{nM}$ solutions of $\mathrm{QD}_{593}$ were prepared under standard aerated $\left(\mathrm{O}_{2}\right)$ and deaerated $\left(\mathrm{N}_{2}\right)$ conditions. Both samples were exposed to continuous UV irradiation using a Xe short arc lamp (150 W, $370 \mathrm{~nm})$. Fresh samples, both aerated and deaerated, were prepared in the presence of $5 \mu \mathrm{M} 5$-HT and emission was collected every 10 mins using a $370 \mathrm{~nm}$ excitation.

The emission intensity of QDs ${ }_{593}$ photoexcited in the presence of 5-HT underwent the expected fluorescence decrease (green, Figure B.5), importantly the rate of fluorescence decrease remained the same in aerated (closed squares) and deaerated (open 
triangles) environments. Both aerated and deaerated solutions of QDs, continuously photoexcited in the absence of 5-HT (blue), initially underwent photoenhancement (likely due to photo-induced annealing of QD surface defects ${ }^{34}$ ) before undergoing a slow decrease in emission intensity. Notably, the rates of QD fluorescence intensity changes remained the same in $\mathrm{O}_{2}$ or $\mathrm{N}_{2}$ atmospheres regardless of the presence of 5-HT.

Therefore, it is unlikely that the emission loss of the QDs is due to interactions with molecular oxygen or oxygen-centered reactive species.

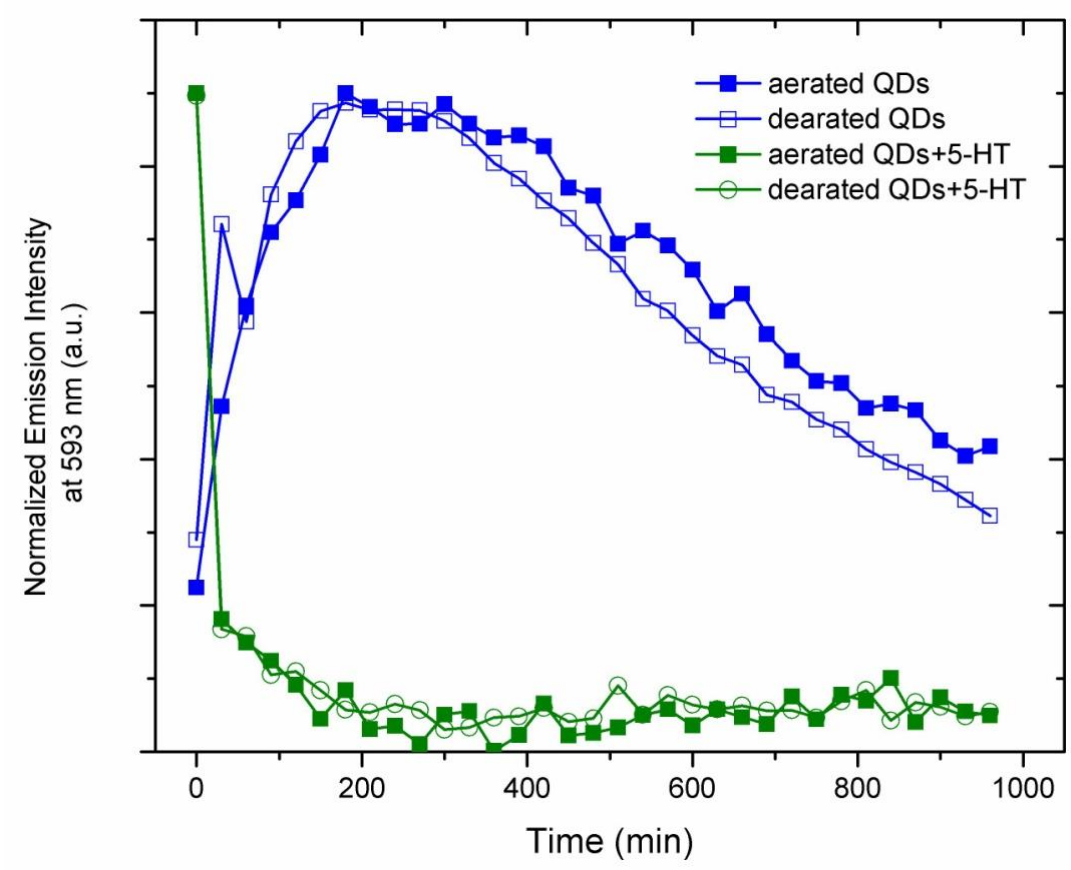

Figure B.5. Emission maxima of $\mathrm{QD}_{593}$ solutions under the irradiated with UV light both in the presence of (green) and without (blue) 5-HT, plotted as a function of time. Under aerated and deaerated (squares and triangles, respectively), the emission intensity exhibits the same trend while undergoing light treatment, suggesting the presence of molecular oxygen or oxygen centered radical species does not affect the emission properties of the QDs. 


\section{Determination of Other Radical Species}

Because radicals have been detected in other solutions of fluorescent QDs, ${ }^{46,} 65$ Electron paramagnetic resonance (EPR) spectroscopy has been employed to investigate the presence of any short-lived intermediate radical species in the 5-HT-QD solutions. Neat 5-HT and $\mathrm{QD}_{655}$ solutions in the presence of the spin trap, 5,5-dimethyl-1-pyrroline N-oxide (DMPO) were prepared. The freshly prepared 5-HT/DMPO solution exhibits a spectrum consistent with radical formation, while the QD/DMPO solution does not (Figure C.6A and B, respectively). After the samples were aged for $24 \mathrm{hrs}$ under ambient conditions, radical signals are clearly observed in the QD solution (orange); over the same aging period, the radical signals from the 5-HT solution have decreased in strength. The hyperfine coupling of the DMPO-radical spectrum (with an apparent 1:2:2:1 quartet), suggests the presence of a DMPO-OH adduct. ${ }^{66}$ 

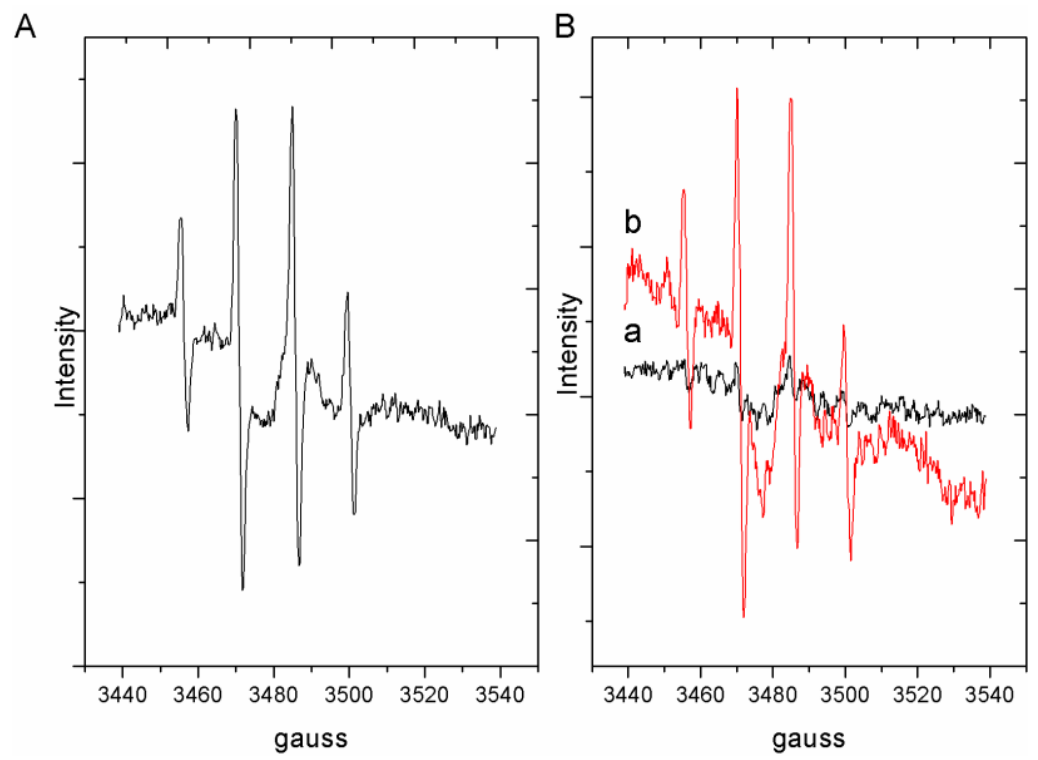

Figure B.6. EPR scans of fresh 5-HT (A) and QDs (B, line a) solutions (in borate buffer) in the presence of the spin trap DMPO. The EPR of the QDs was also collected after $1 \mathrm{hr}$ of sitting at room temperature.

The EPR data suggests that the DMPO-OH adduct is formed occurs after the QDs are aged at room temperatures. The QD emission intensity decreases faster at room temperature, so it is possible that the formation $\mathrm{OH}$ radicals facilitate this reaction.

\section{Investigation as to the Possible Decomposition of 5-HT}

This investigation aims to help determine if a PCT between 5-HT and QDs generate any organic products. Neat 5-HT exhibits emission at $425 \mathrm{~nm}$ when excited at $380 \mathrm{~nm}$, owing to its conjugated ring system, as shown in Figures B.7A and B. When $\mathrm{QD}_{655}$ are added, QD emission is observed. After 30 mins of irradiation, the solution of $\mathrm{QDs}_{655}+5-\mathrm{HT}$ no longer exhibit the $425 \mathrm{~nm}$ peak, and the peak at 655 is clearly diminished, indicating the quenching of both $\mathrm{QD}_{655}$ and 5-HT fluorescence. The absence of a 5-HT peak in the irradiated sample indicates either a chemical change disrupting the 
aromatic bonds of the 5-HT structure has occurred, or there exists an energy or charge transfer reaction. To investigate possible 5-HT structural changes, ${ }^{1} \mathrm{H}$ NMR was used to analyze the solutions from Figure B.7 A. Serotonin has 7 protons which give clear NMR signals; Figure B.7 C and D show the ${ }^{1} \mathrm{H}$ NMR spectra for neat 5-HT only (red), 5-HT+ $\mathrm{QDs}_{655}$ (blue) and irradiated 5-HT + QDs 655 (green), along with spectral assignments. Curiously, there is no change in 5-HT structure detectable via NMR.

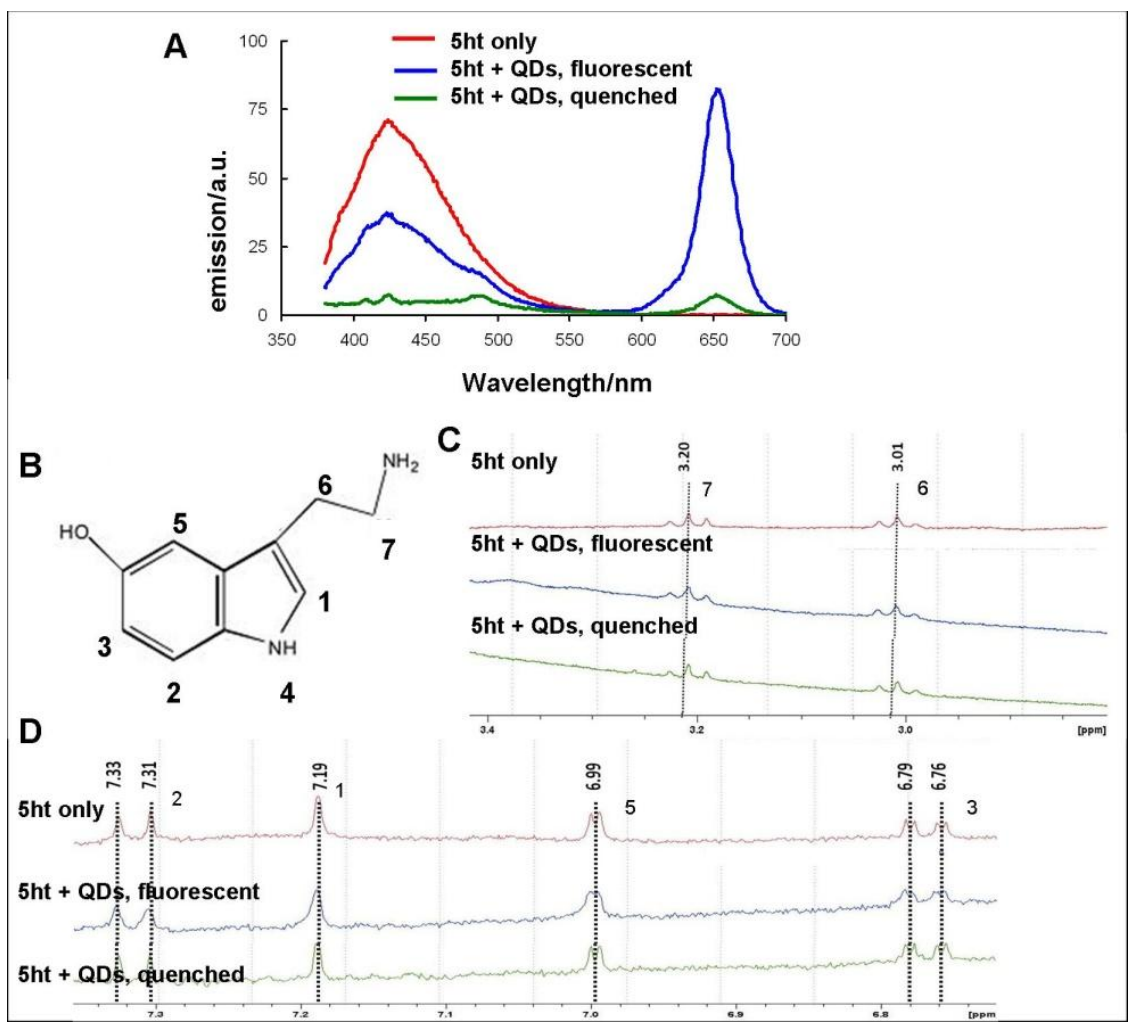

Figure B.7 (A) Emission spectra of 5-HT (red), 5-HT + QDs ${ }_{655}$ solution (blue), and irradiated 5-HT+ QD solution (green) using 380nm excitation. (B) Serotonin molecule. (C and D). ${ }^{1} \mathrm{H}$ NMR spectra (in $\mathrm{D}_{2} \mathrm{O}$ ) of the same solutions shown in Figure 2.8A. The protons in these spectra are labeled in accordance with (B), 2.8-3.4 and 6.6-7.4 ppm, respectively) 
The lack of structural changes in the ${ }^{1} \mathrm{H}$ NMR suggests that intact 5-HT molecules still exist in solution after the light treatment. While these ${ }^{1} \mathrm{H}$ NMR signals could be kinetics-related, (5-HT may have a faster rate of transfer than the $400 \mathrm{MHz}$ can detect), these intact 5-HT molecules would still be expected to be luminescent. Since luminescence is not observed after the QD addition and light treatment, it seems plausible that a radical, non-luminescent 5-HT species may also exist in solution. 


\section{Supporting Data for the Zintl Salt Metathesis of $\mathrm{NaSi}$ and $\mathrm{NH}_{4} \mathrm{Br}$}

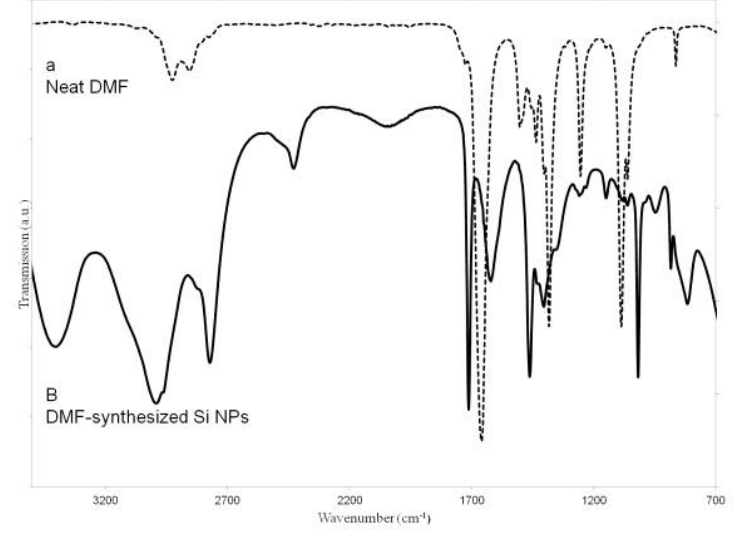

Figure C.1. Full FTIR spectra of neat DMF (line a, dashed) and DMF-synthesized Si NPs (line b, solid).

A

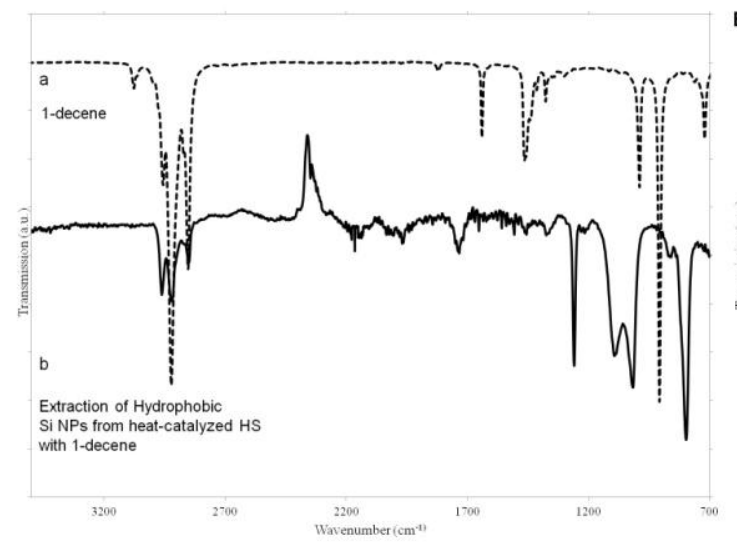

B

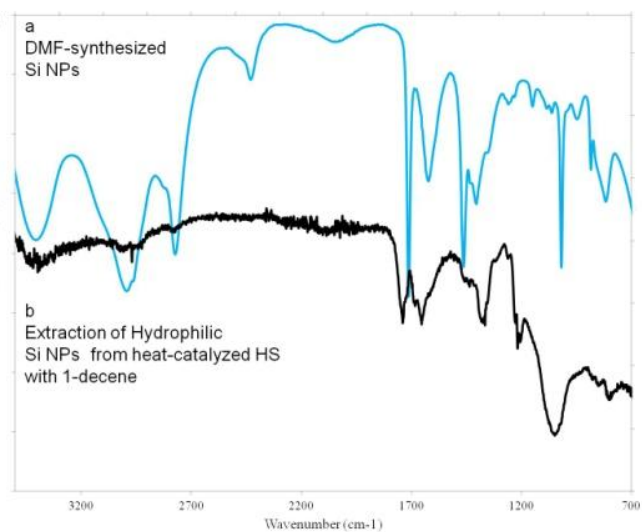

Figure C.2. Full FTIR spectra of the water/hexane extractions from the heat-catalyzed HS of the DMF-synthesized Si NPs with 1-decene. (A) Neat 1-decene (line a, dashed) compared to the extracted hydrophobic layer (line b, solid) of Si NPs; (B) The DMF-synthesized Si NP spectrum (line a, blue solid) compared to the extracted hydrophilic layer (line b, solid black) of Si NPs. 


\section{Heat-Catalyzed HS of Si NPs using Propargylamine in DMF and Xylene}

To avoid undesired surface interactions and ideally, leave more $\mathrm{Si}-\mathrm{H}$ surface sites for subsequent HS reactions, the Si NPs were also produced in a non-coordinating solvent. Because the heat-catalyzed HS may more efficiently initiate the reaction between $\mathrm{Si}-\mathrm{H}$ surfaces and unsaturated hydrocarbons, a non-coordinating solvent was also used as a solvent to prevent the solvent from interacting with the Si NPs. o-Xylene was selected because its boiling point $\left(144^{\circ} \mathrm{C}\right)$ is comparable to that of DMF $\left(155^{\circ} \mathrm{C}\right)$. The reaction using xylene was performed following the reaction scheme in Fig. 4.1

The hydrophilic Si NPs from the DMF-syntheses appeared to possess the most complicated surface chemistries. For potential biological applications of Si NPs, this is especially problematic; ideally, hydrophilic surface groups, including amines, carboxylic acids, and thiols, would be covalently bound to the surface of Si NPs, as they are capable of subsequent conjugation to biologically relevant molecules. To determine if ligands to deliberately produce functionalized hydrophilic Si NPs could successfully undergo HS, the as prepared $\mathrm{Si}-\mathrm{H}$ NPs underwent HS reaction with the unsaturated hydrocarbon, propargylamine, in the presence of DMF and xylene.

Heat-Catalyzed HS using Propargylamine: The precursor Si-H NPs were brought up in $25 \mathrm{~mL}$ of DMF or xylene. While stirring, $2 \mathrm{~mL}$ of neat propargylamine was added via syringe and the solution was refluxed for $12 \mathrm{hrs}$. A water/hexanes extraction was used to separate the Si NPs from the solvent. The extracted layers were rota-vapped and dispersed in $5 \mathrm{~mL}$ of water or hexanes, respectively. 
The hydrophilic Si NP layers from the HS of the as-prepared Si NPs in DMF and xylene were examined by FT-IR to determine if the HS reactions were successful, or if complex Si NP surface terminations still existed. Shown in Figure C.3A, the propargylamine-synthesized Si NPs in DMF did exhibit two carbonyl stretches $\left(1740 \mathrm{~cm}^{-}\right.$ ${ }^{1}$ and $1652 \mathrm{~cm}^{-1}$ ), but contained stretches consistent with propargylamine, including shoulders at $1589 \mathrm{~cm}^{-1}, 866 \mathrm{~cm}^{-1}$, and $1021 \mathrm{~cm}^{-1}$, representing the two N-H stretches and $\mathrm{C}-\mathrm{N}$ stretch, respectively. Potential new peaks are observed at $1220 \mathrm{~cm}^{-1}$ indicative of Si$\mathrm{C}$ and $816 \mathrm{~cm}^{-1}$ indicative of Si-N. Additionally, the $\mathrm{C} \equiv \mathrm{C}\left(2116 \mathrm{~cm}^{-1}\right)$ and $\mathrm{CH} \equiv \mathrm{C}(3292$ $\mathrm{cm}^{-1}$ ) stretches present in neat propargylamine are absent from the FT-IR spectrum of the propargylamine-synthesized Si NPs. Taken together this suggests that the hydrophilic, propargylamine-synthesized $\mathrm{Si} \mathrm{NP}$ prepared in DMF possess both DMF and propargylamine associated surface groups.

A

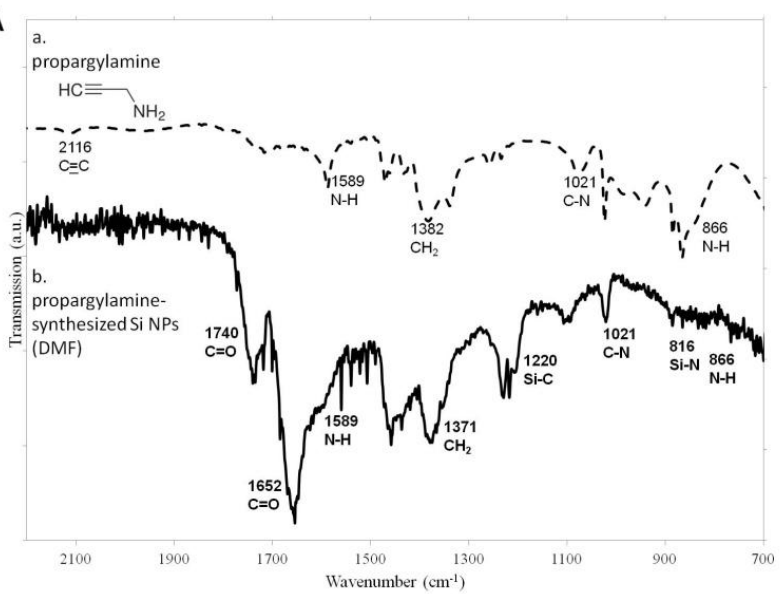

B

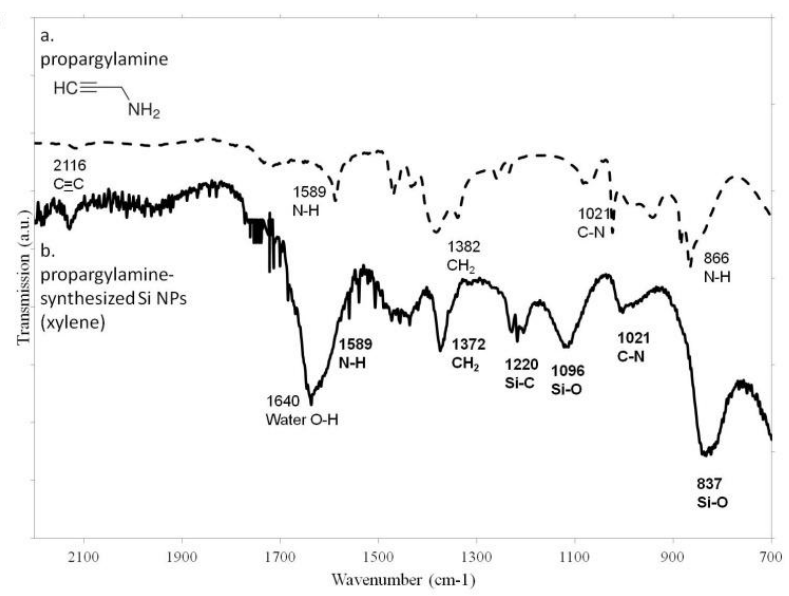

Figure C.3. (A) FT-IR spectra of (a) neat propargylamine (dashed) and (b) propargylaminesynthesized Si NPs. (solid) in DMF. (B) FTIR spectra of (a) neat propargylamine (dashed) and (b) propargylamine-synthesized Si NPs (solid) in xylene. 
The propargylamine-synthesized Si NPs prepared in xylene was also examined by FT-IR, as shown in Fig C.3B. These particles exhibited a stretch around $1220 \mathrm{~cm}^{-1}$, consistent with $\mathrm{Si}-\mathrm{C}$ and a successful HS between the hydride-termination of the asprepared $\mathrm{Si}$ NPs from $\mathrm{DMF}$ and propargylamine. The $\mathrm{C} \equiv \mathrm{C}$ and $\mathrm{CH}=\mathrm{C}$ stretches were also not present in the Si NP sample, supporting the HS. Additionally stretches belonging to propargylamine are present, including $\mathrm{C}-\mathrm{N}$ and $\mathrm{N}-\mathrm{H}$ at 1589 and $1021 \mathrm{~cm}^{-1}$; the $\mathrm{N}-\mathrm{H}(\mathrm{s})$ at $866 \mathrm{~cm}^{-1}$ (from neat propargylamine) and potential $\mathrm{Si}-\mathrm{N}(\mathrm{s})$ are potentially obscured by the $\mathrm{Si}-\mathrm{O}(\mathrm{b})$ at $837 \mathrm{~cm}^{-1}$. There is also surface oxidation at $1096 \mathrm{~cm}^{-1}$, likely due to the oxidation of the NP surface from water. (Water is also responsible for the large stretch at $1640 \mathrm{~cm}^{-1}$.) No xylene solvent peaks were observed in the FTIR.

The UV spectra of the hydrophilic Si NPs prepared from the HS with propargylamine in DMF and xylene are shown in Figure C.4A. Both samples exhibit an increase in absorbance at about $500 \mathrm{~nm}$ with an absorbance peak at about $260 \mathrm{~nm}$. This suggests that the Si NPs produced by the metathesis method have similar physical characteristics by exhibiting both direct- and indirect-related absorbance.

The PL emission spectra of the propargylamine-synthesized Si NPs in DMF and xylene were compared (Fig. C.4.11B). The propargylamine-synthesized Si NPs prepared in DMF have highly intense emission when excited with UV light (excitation wavelengths ranging from 340-400 nm). Similar to previous emission spectra, these Si NPs exhibit an increasing emission maximum wavelength with increasing excitation wavelength, consistent with a polydisperse NP sample. The propargylamine-synthesized Si NPs prepared in xylene exhibit emission at almost identical wavelengths, but with 
significantly lower emission intensity. Because there particles are similar in size (Figure C.5) particle size has little effect o the emission. However, the emission decrease may be due to the increased surface oxide on the xylene-synthesized Si NPs. This decrease in emission has been observed in the presence of surface oxidation. ${ }^{7 \mathrm{a}, 21}$ The presence of SiO has been hypothesized to provide alternate recombination pathways for excitons generated within the crystalline Si core. If excitons recombinant the surface defect sites, or dangling bonds within the surface oxide, then this could result in the decrease in the number of recombination events which give rise to the observed emission. This may explain the decreased emission intensity of the propargylamine-synthesized Si NPs prepared in xylene (as compared to those prepared in DMF.)

A

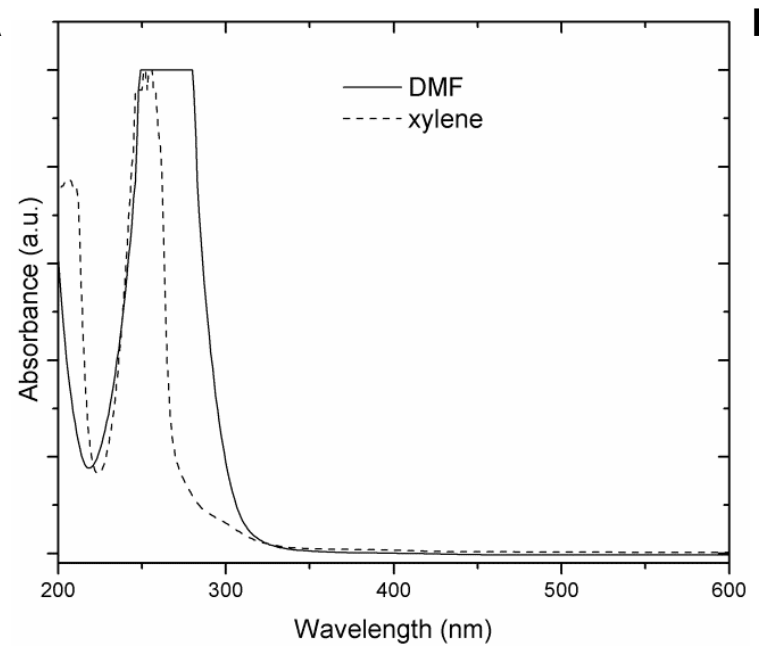

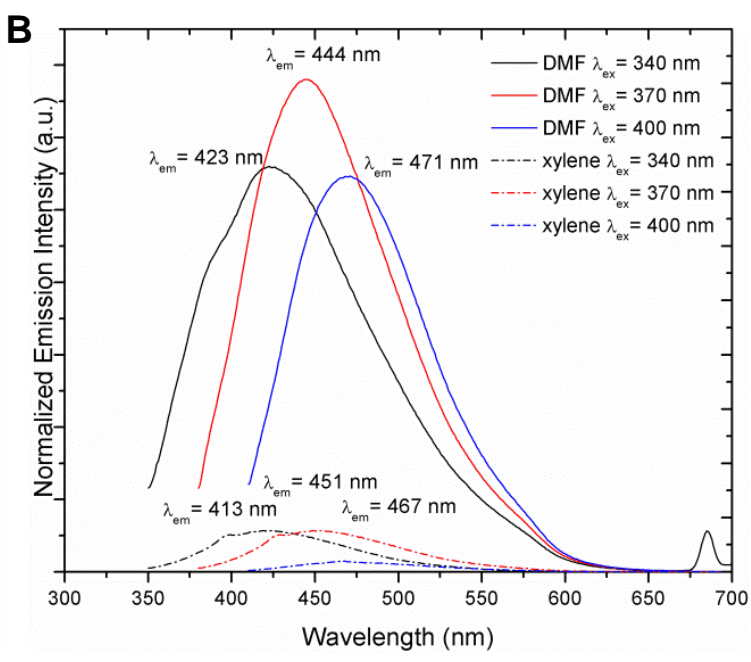

Figure C.4. Comparison of the optical properties of the propargylamine-synthesized Si NPs prepared in DMF and xylene. (A) UV spectra of the propargylamine-synthesized Si NPs prepared in DMF-(solid) and xylene (dashed). (B) PL spectra of the propargylamine-synthesized Si NPs prepared in DMF (solid lines) and xylene (dashed lines) at various excitation wavelengths, $\lambda_{\mathrm{ex}}=340$ (black), $\lambda_{\mathrm{ex}}=370$ (red), $\lambda_{\mathrm{ex}}$ $=400$ (blue) 

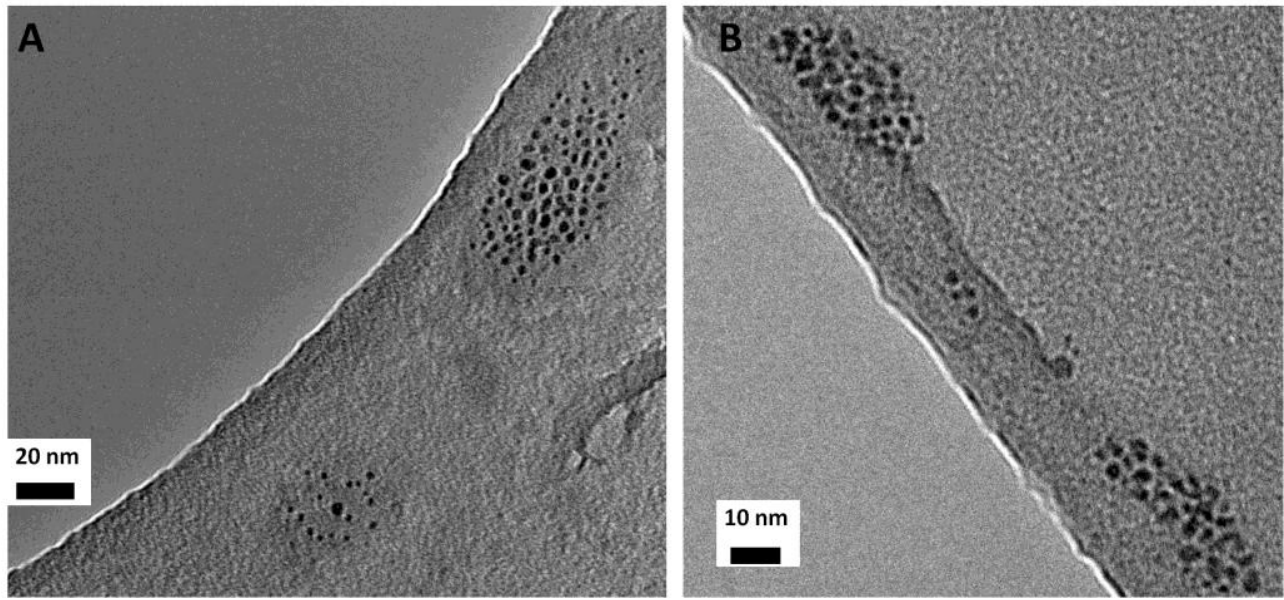

Figure C.5. TEM image of propargylamine-synthesized Si NPs in (A) DMF and (b) xylene.

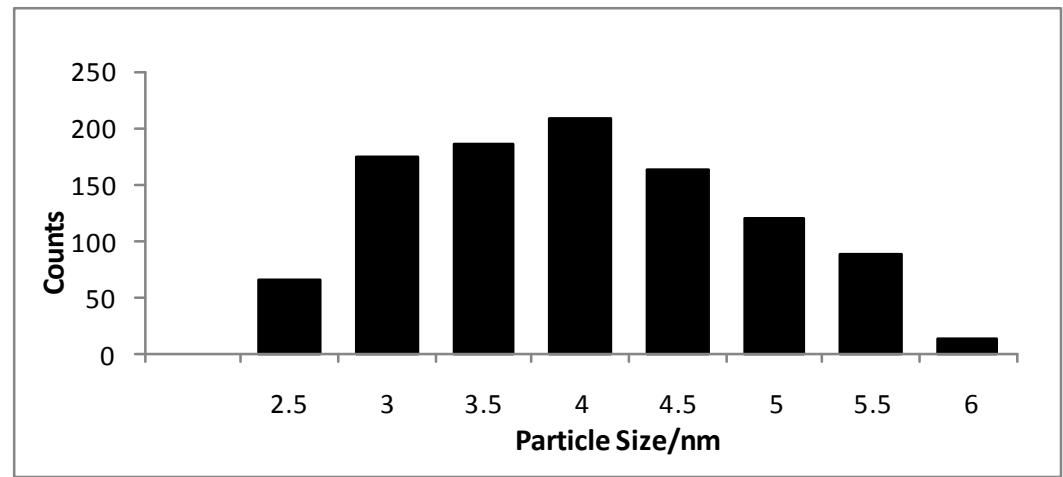

Figure C.6. Particle size histogram for GA-synthesized Si NPs based on a survey of 1020 NPs measured in different regions of the TEM grid was found to be $3.9 \pm 0.8 \mathrm{~nm}$. 


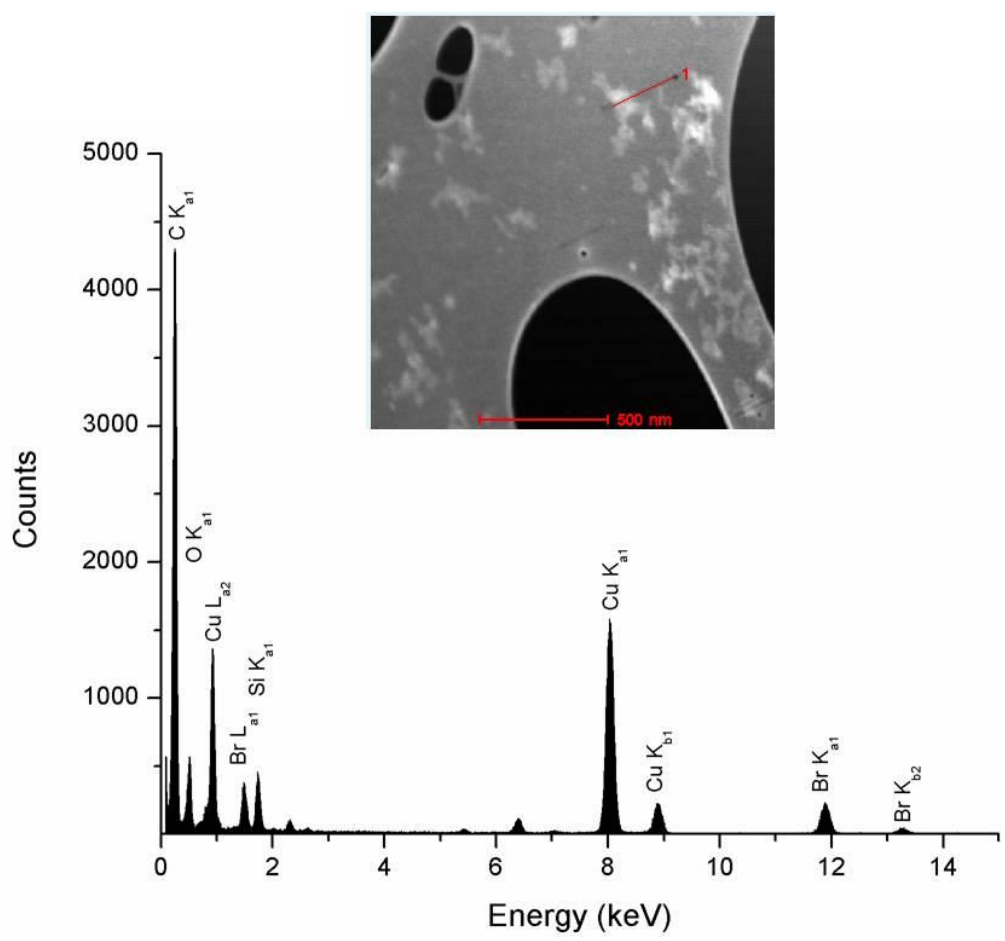

Figure C.7. EDX spectrum of a GA-synthesized Si NP sample revealing the elements $\mathrm{Si}, \mathrm{O}, \mathrm{C}, \mathrm{Cu}$ (grid), $\mathrm{Br}$, and $\mathrm{Na}$ (unlabeled).

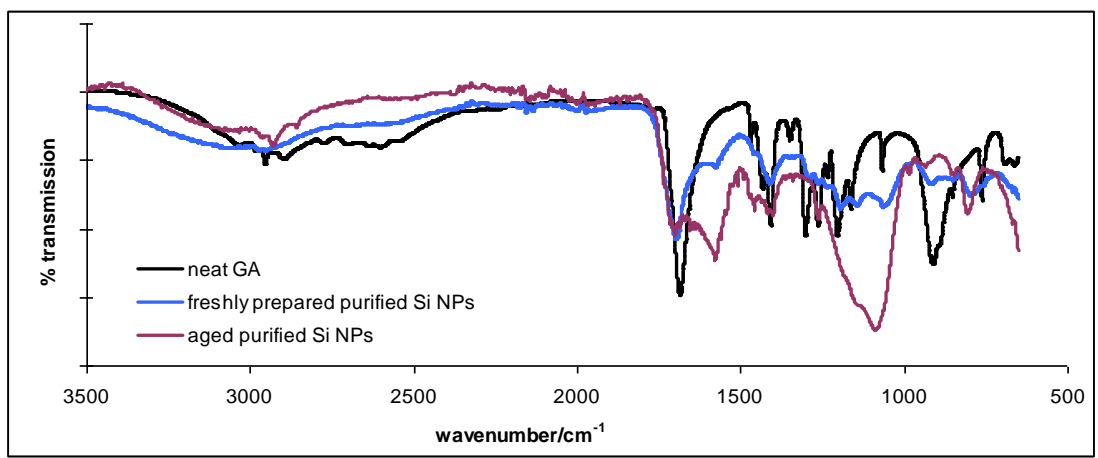

Figure C.8.FT-IR spectra of the freshly purified (blue) and aged purified GA-synthesized Si NPs alongside the FT-IR spectrum of neat GA (black). 

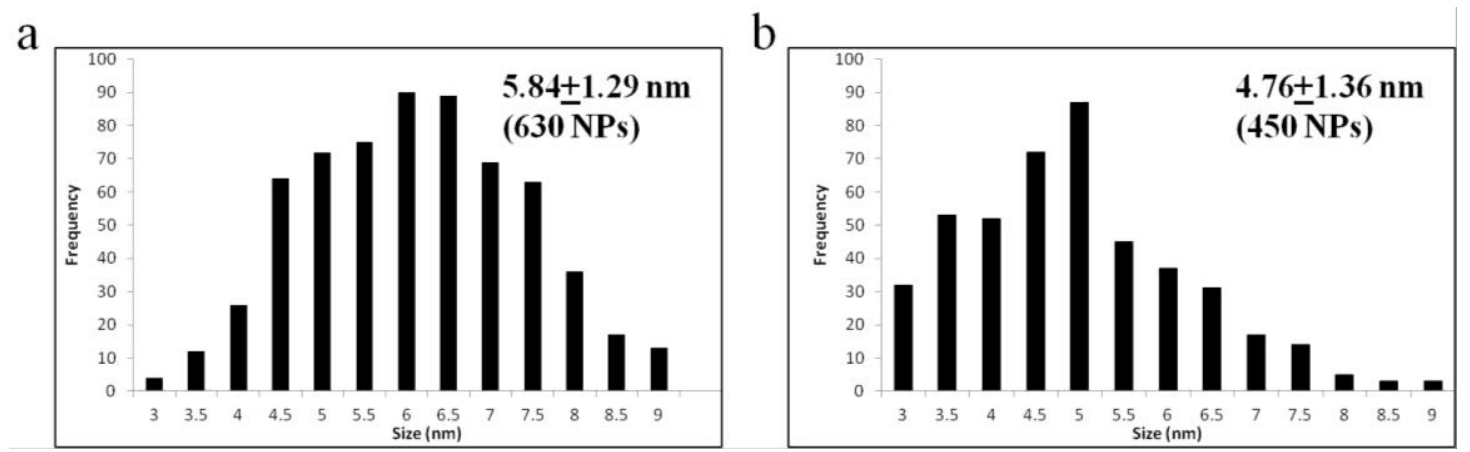

Figure C.9. Particle size histogram for (a) CA-synthesized and (b) en-synthesized Si NPs.

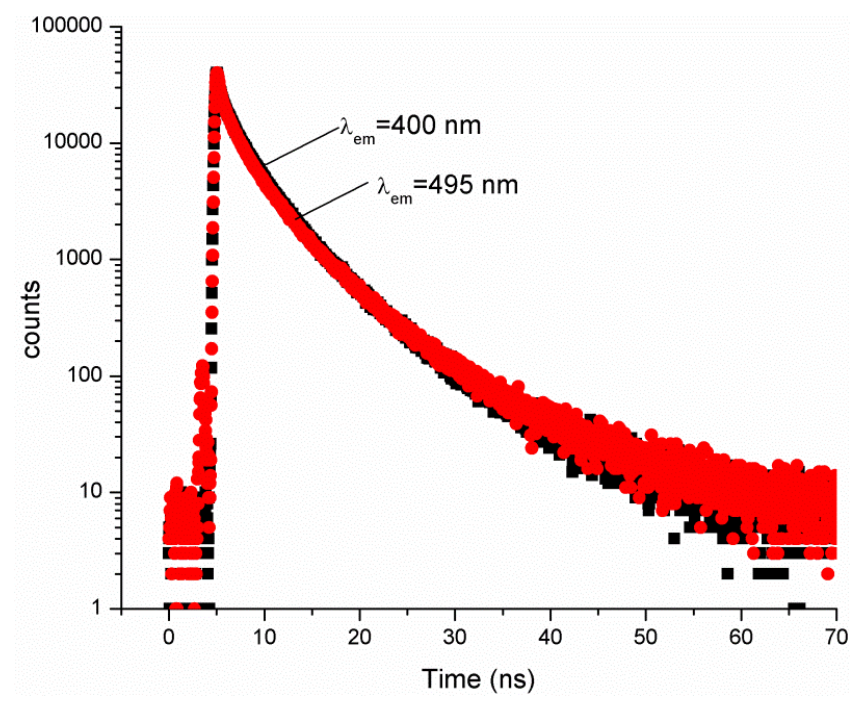

Figure C.10. Time-resolved emission spectrum of GA-synthesized Si NPs in $\mathrm{H}_{2} \mathrm{O}$ excited at $375 \mathrm{~nm}$ with the emission monitored at $400 \mathrm{~nm}$ (black) and $495 \mathrm{~nm}$ (red); the intensity weighted lifetime was determined to be 3.2 and $3.7 \mathrm{~ns}$, respectively. 


\section{Other One-pot iterations}

Glutathione (GSA)

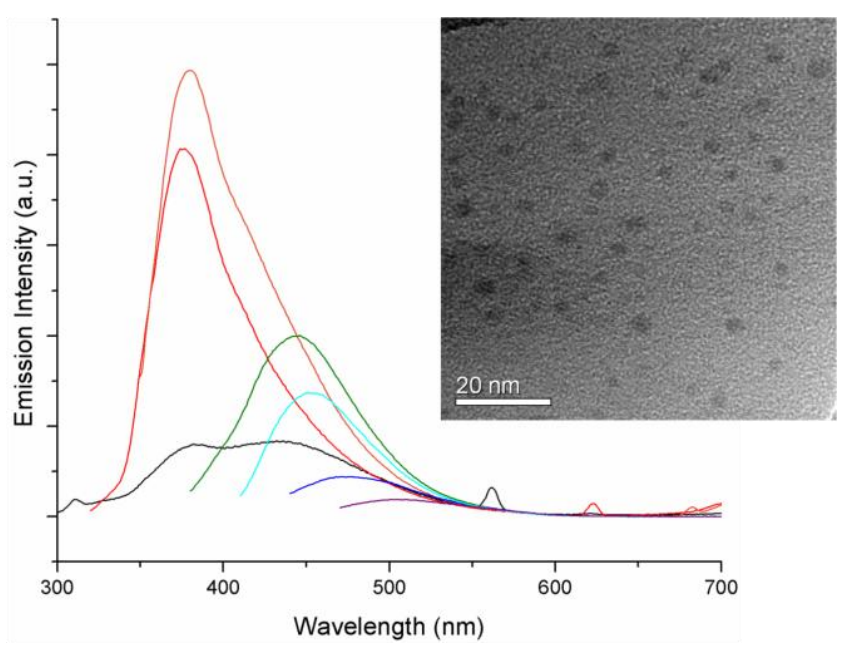

Figure C.11. Emission spectra $\left(\lambda_{\mathrm{ex}} 280-460 \mathrm{~nm}\right)$ and TEM image of GSA-synthesized Si NPs in water.

Polyethylene glycol (PEG)

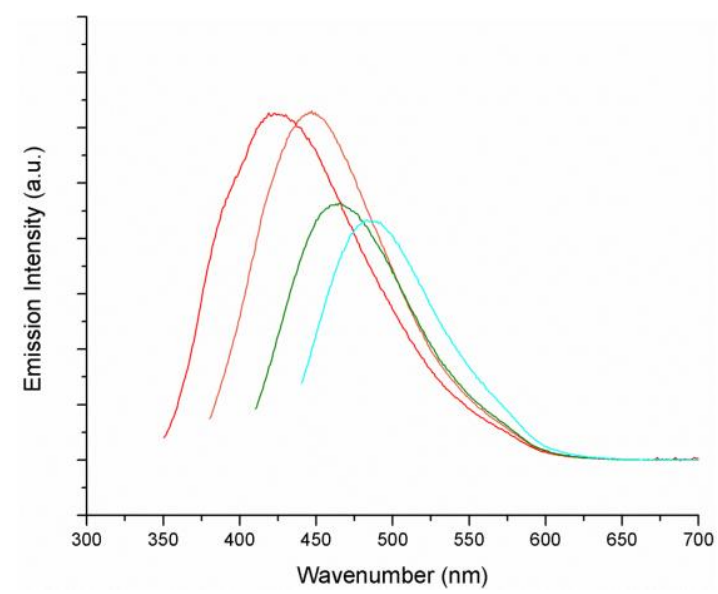

Figure C.12. Emission spectra $\left(\lambda_{\mathrm{ex}} 340-430 \mathrm{~nm}\right)$ of the PEG-synthesized Si NPs in water. 
Additional Metal Ions and Molecular Quenching of Si NP Emission

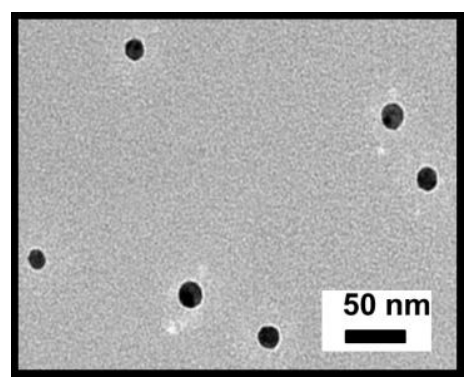

Figure D.1. TEM image of the products from the photoreductionof $\mathrm{HAuCl}_{4}{ }^{-}+\mathrm{GA}_{-\mathrm{Si}} \mathrm{NPs}$ after 5 hrs of ambient exposure.

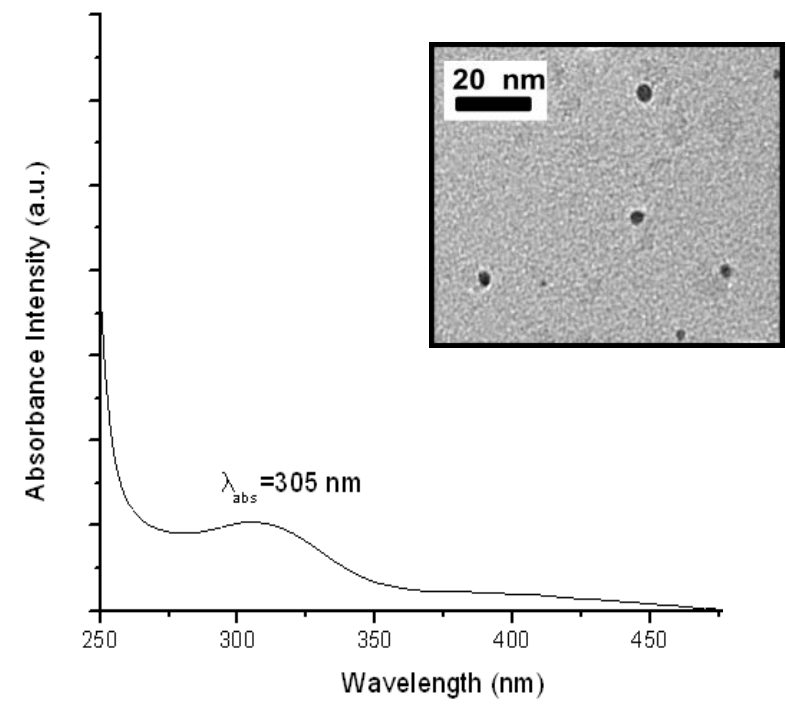

Figure D.2. $\mathrm{HAuCl}_{4}{ }^{-}+$neat GA after 3 hrs of $\mathrm{UV}$ irradiation.

The formation of Au NPs prepared in the presence of GA-Si NPs and UV light exhibited some exhibited some pH dependency. The Au NP SPR band was bluer shifted in the presence of $1.0 \mathrm{M} \mathrm{HCl}$, as compared to the SPR band in $1.0 \mathrm{M} \mathrm{NaOH}$ (Figure 
D.3A and B). Not only is the band more blue shifted, but it also forms faster, suggesting the particles form more readily in acidic conditions.
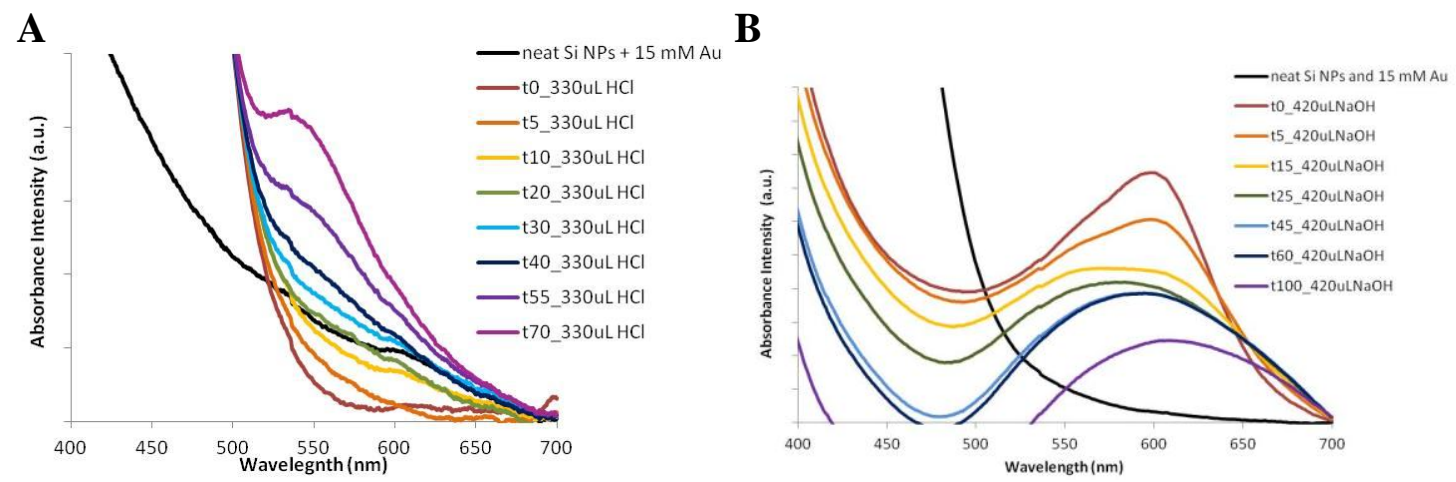

Figure D.3. pH dependency on the formation of Au NPs in the presence of GA-Si NPs. ( A.) Addition of $1.0 \mathrm{M} \mathrm{HCl}$; (B) Addition of 1.0 M NaOH

This $\mathrm{pH}$ dependence may be explained by referencing the poudbaix diagram of gold, though experiments are still ongoing (Figure D.4). Pourbaix diagrams indicate the $\mathrm{pH}$ and potential $(\mathrm{eV})$ conditions under which particular species are expected to be form; the dotted lines represent the limits of stability of water. 


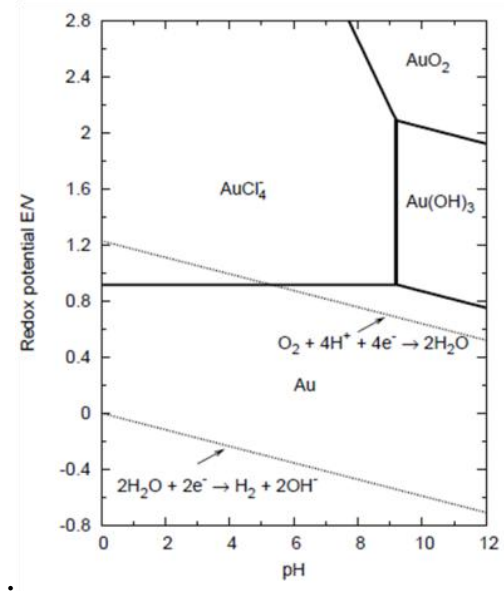

Figure D.4. Pourbaix diagram of Au.

The red $\mathrm{SiO}_{\mathrm{x}}-\mathrm{Si}$ NPs were also exposed to $0.5 \mathrm{M} \mathrm{CuSO}_{4}$ (Figure D.5). After 85 mins of UV-irradiation, no SPR band corresponding to $\mathrm{Cu}$ NPs was observed. However, after 1.0 M NaOH was added, a possible SPR band was observed around $430 \mathrm{~nm}$.

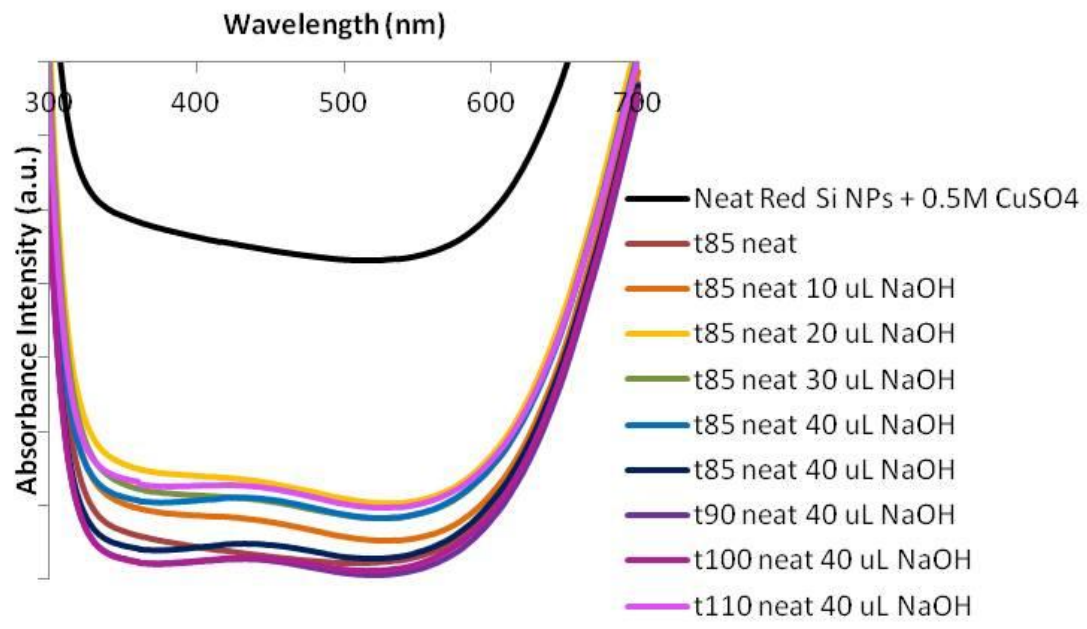

Figure D.5. Absorption spectrum of $\mathrm{CuSO}_{4}$ in the presence of red $\mathrm{SiO}_{\mathrm{x}}-\mathrm{Si} \mathrm{NPs}$. A potential SPR band was observed after the addition of $1.0 \mathrm{M} \mathrm{NaOH}$. 


\section{Additional Ions}

Other metal ions, including $\mathrm{Al}^{3+}, \mathrm{Bi}^{3+}, \mathrm{Cr}^{3+}$, and $\mathrm{Fe}^{3+}$ were added to the GA-Si NPs. Both the emission and absorption spectra were monitored over time. While the absorption spectra of the metal ions showed evidence of decreasing absorption alongside GA-Si NP quenching, no SPR bands were formed.
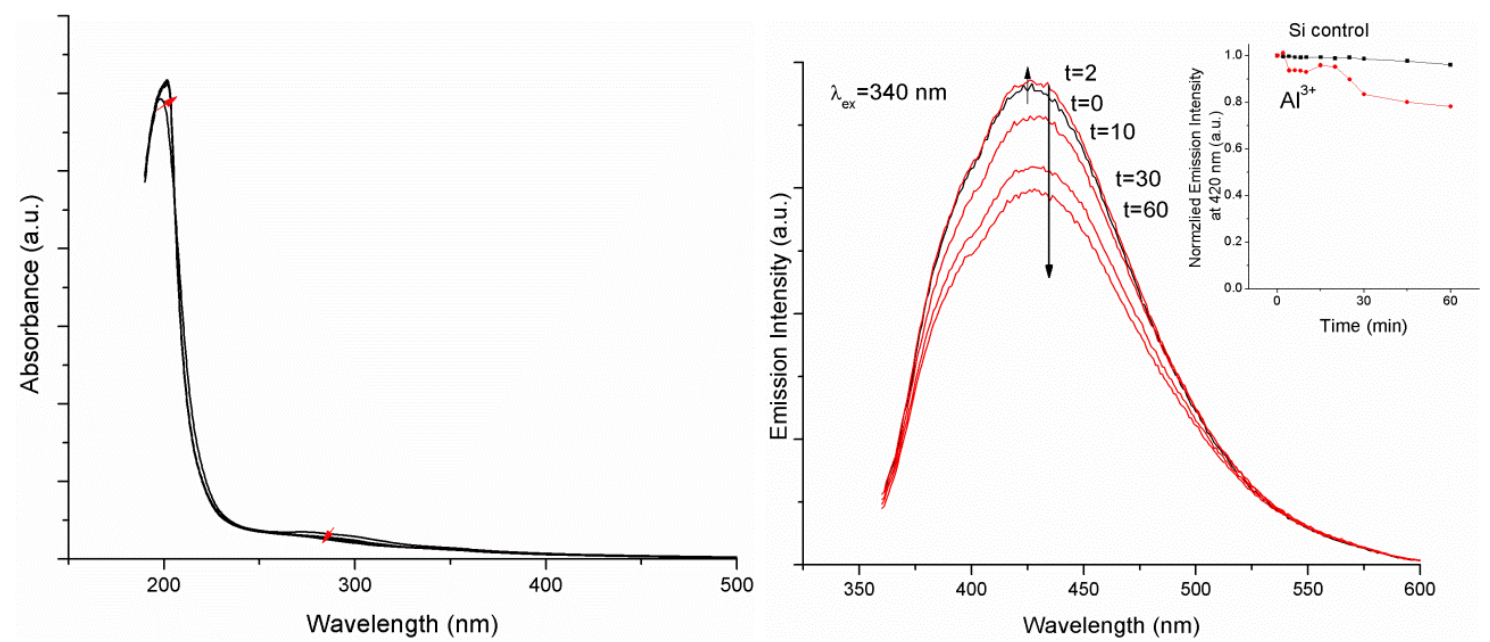

Figure D.6. Absorbance and Emission $\left(\lambda_{\mathrm{ex}}=340 \mathrm{~nm}\right)$ spectra after the immediate addition of $1 \mathrm{M} \mathrm{Al}^{3+}$ to GA-Si NPs in $\mathbf{n H}_{2} \mathbf{O}$; Inset. Normalized emission illustrating the potential photoenhancement and quench of the GA-Si NPs emission in the presence of $\mathrm{Al}^{3+}$. 

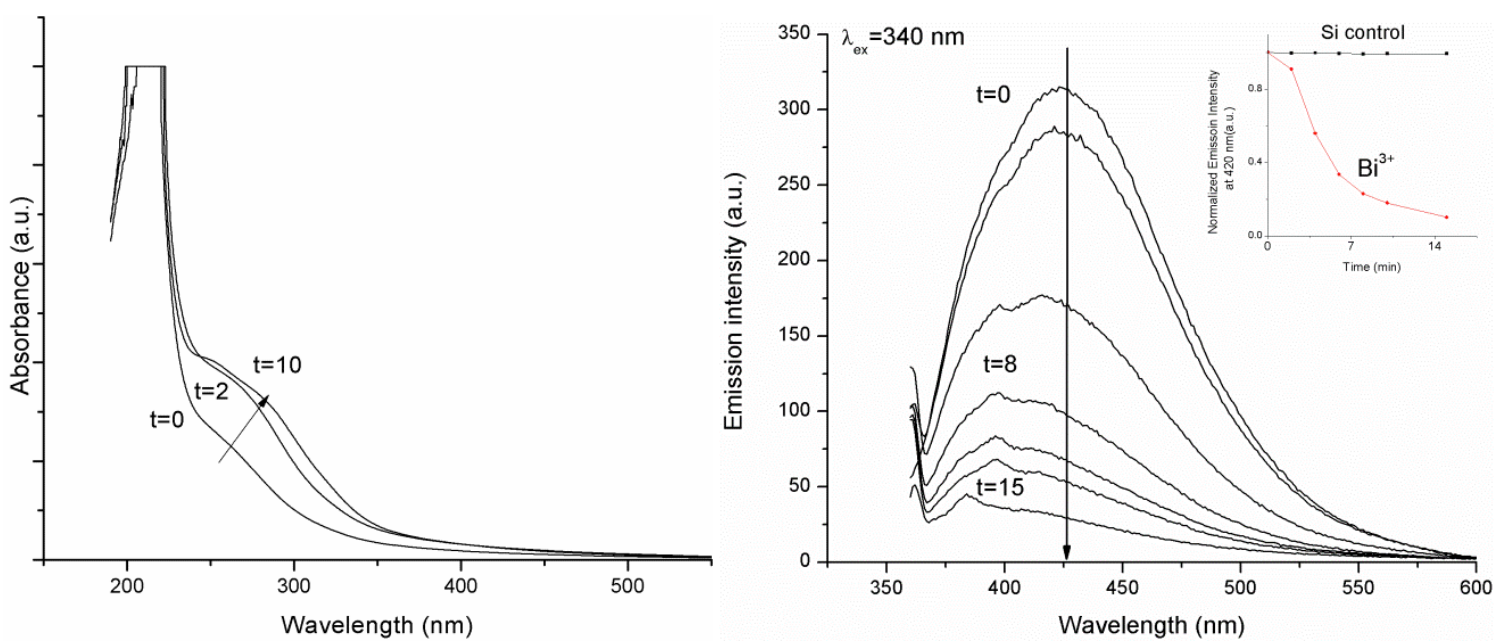

Figure D.7. Absorbance and Emission $\left(\lambda_{\mathrm{ex}}=340 \mathrm{~nm}\right)$ spectra after the immediate addition of $0.1 \mathrm{mM}$ $\mathbf{B i}^{3+}$ to GA-Si NPs in $\mathbf{n H}_{2} \mathbf{O}$; Inset. Normalized emission illustrating the fast quench of the GA-Si NPs emission in the presence of $\mathrm{Bi}^{3+}$.
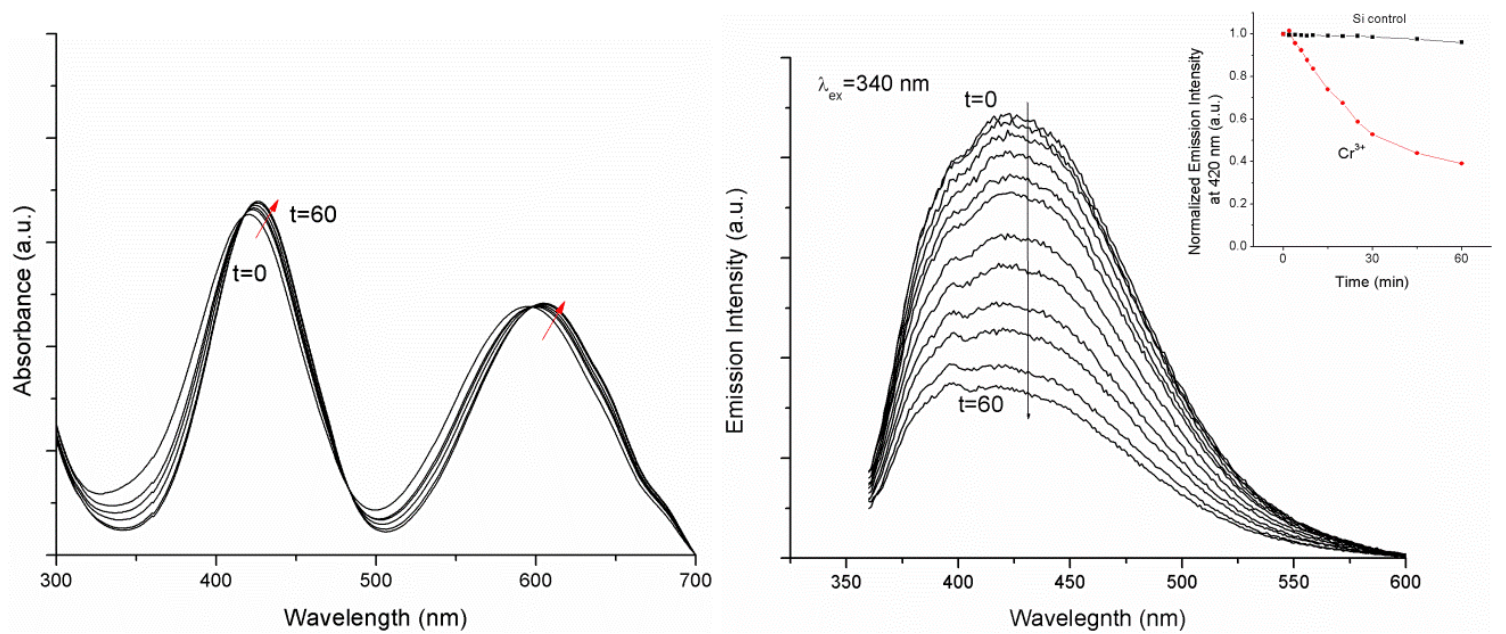

Figure D.8. Absorbance and Emission $\left(\lambda_{\mathrm{ex}}=340 \mathrm{~nm}\right)$ spectra after the immediate addition of $100 \mathrm{mM}$ $\mathrm{Cr}^{3+}$ to GA-Si NPs in $\mathbf{n H}_{2} \mathbf{O}$; Inset. Normalized emission illustrating the quench of the GA-Si NPs emission in the presence of $\mathrm{Cr}^{3+}$. 

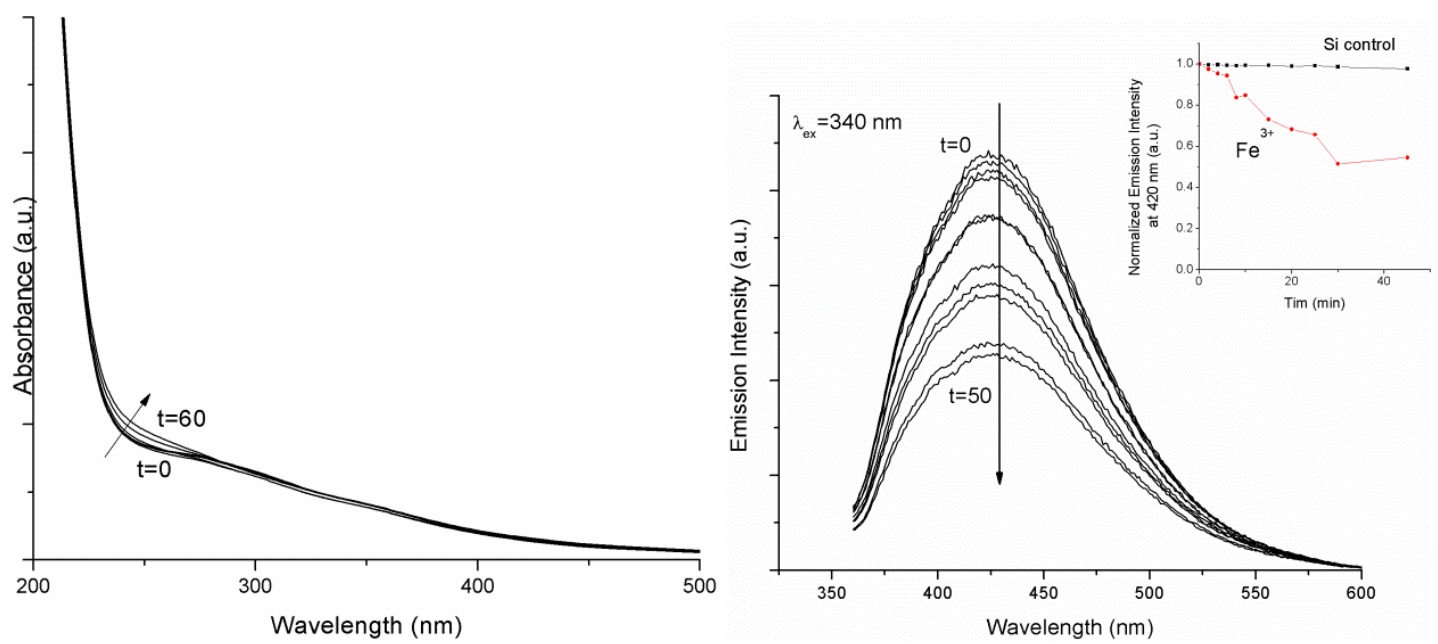

Figure D.9. Absorbance and Emission $\left(\lambda_{\mathrm{ex}}=340 \mathrm{~nm}\right)$ spectra after the immediate addition of $0.5 \mathrm{mM}$ $\mathbf{F e}^{3+}$ to GA-Si NPs in $\mathbf{n H}_{2} \mathbf{O}$; Inset. Normalized emission illustrating the quench of the GA-Si NPs emission in the presence of $\mathrm{Fe}^{3+}$.

\section{Amine Quenchers}

Because amines have been used as quenching analytes for porous and nanosized Si, they have also been added to the GA-Si NPs.

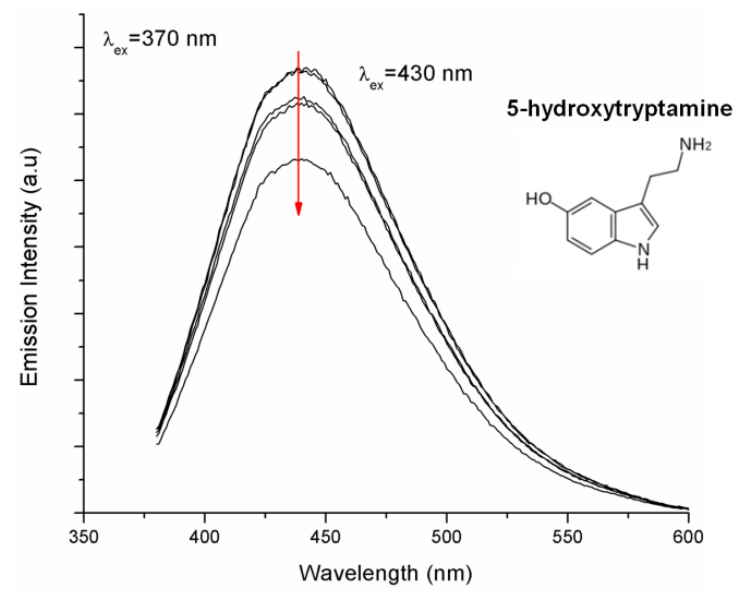

Figure D.10. Emission $\left(\lambda_{\mathrm{ex}}=370 \mathrm{~nm}\right)$ after the immediate addition of $0.2 \mathrm{~mL} 4.5 \mathrm{mM}-.4 \mathrm{M}$ 5ht $(0.2$ mL) 5-hydroxytryptamine (5-HT) to $0.8 \mathrm{~mL}$ GA-Si NPs in $\mathbf{n H}_{2} \mathrm{O}$. 


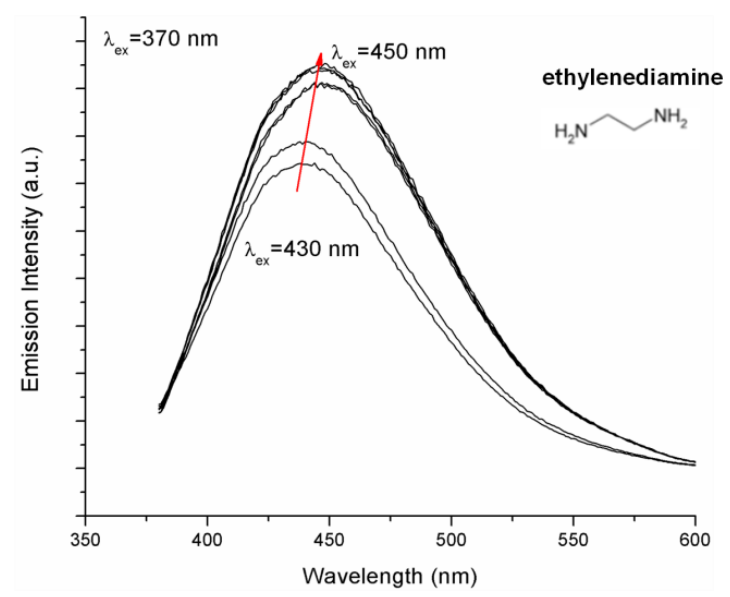

Figure D.11. Emission $\left(\lambda_{\mathrm{ex}}=370 \mathrm{~nm}\right)$ after the immediate addition of $0.2 \mathrm{~mL} 0.05 \mathrm{M}-0.5 \mathrm{M}$ ethylenediamine to $0.8 \mathrm{~mL}$ GA-Si NPs in $\mathrm{nH}_{2} \mathrm{O}$.

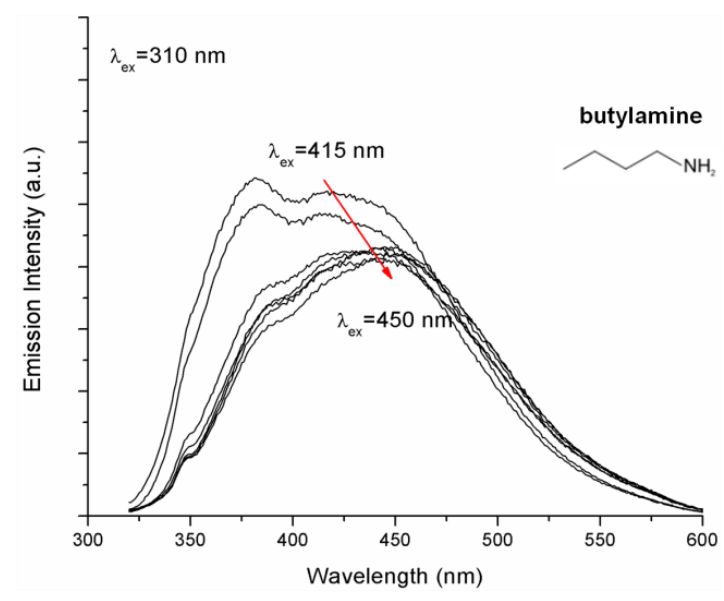

Figure D.12. Emission $\left(\lambda_{\mathrm{ex}}=310 \mathrm{~nm}\right)$ after the immediate addition of $0.2 \mathrm{~mL} .01 \mathrm{M}-.5 \mathrm{M}$ butylamine to $0.8 \mathrm{~mL}$ GA-Si NPs in $\mathrm{nH}_{2} \mathrm{O}$. 


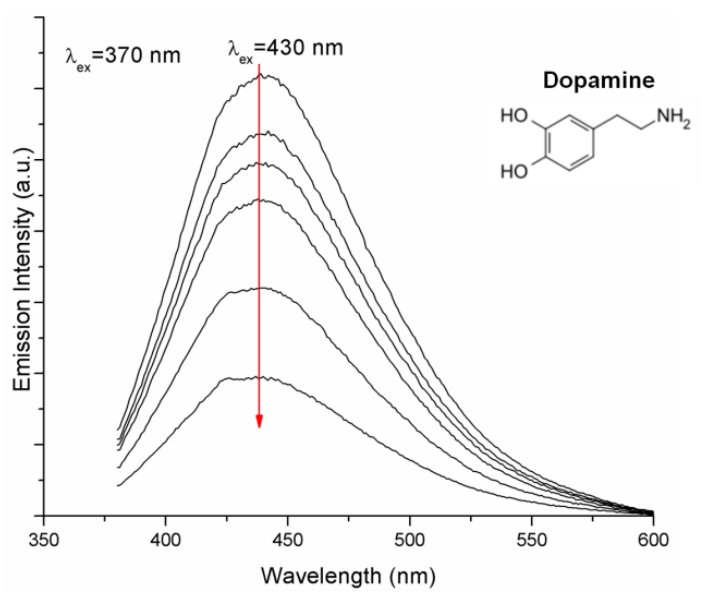

Figure D.13. Emission $\left(\lambda_{\mathrm{ex}}=370 \mathrm{~nm}\right)$ after the immediate addition of $0.2 \mathrm{~mL} \mathrm{0.05} \mathrm{M}-0.5 \mathrm{M}$ dopamine to $0.8 \mathrm{~mL}$ GA-Si NPs in $\mathrm{nH}_{2} \mathrm{O}$.

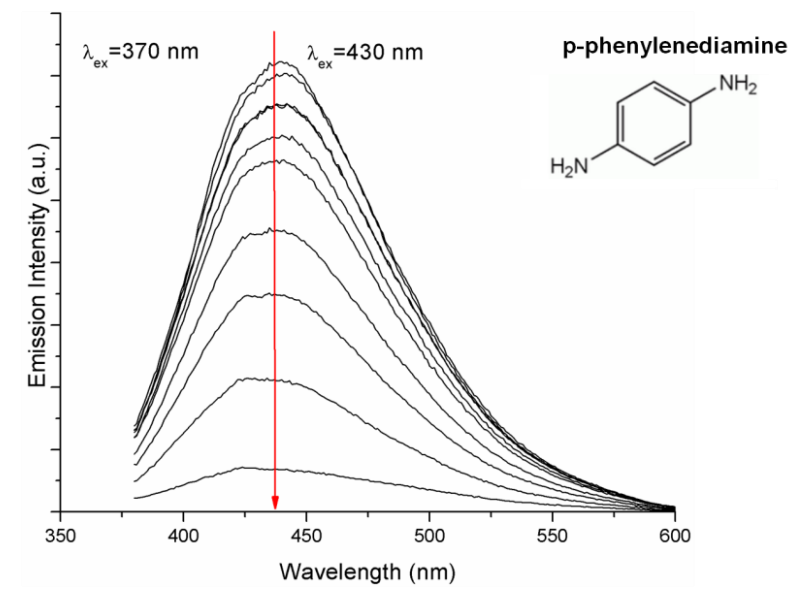

Figure D.14. Emission $\left(\lambda_{\mathrm{ex}}=370 \mathrm{~nm}\right)$ after the immediate addition of $0.2 \mathrm{~mL} 0.005 \mathrm{M}-0.5 \mathrm{M}$ phenylenediamine to $0.8 \mathrm{~mL}$ GA 
Full Characterization of the Ester-Functionalized Si NPs

\section{E.1 Analytical Characterization of Prepared Esters}

\section{E.1.1 Methyl-4-pentenoate (5M)}

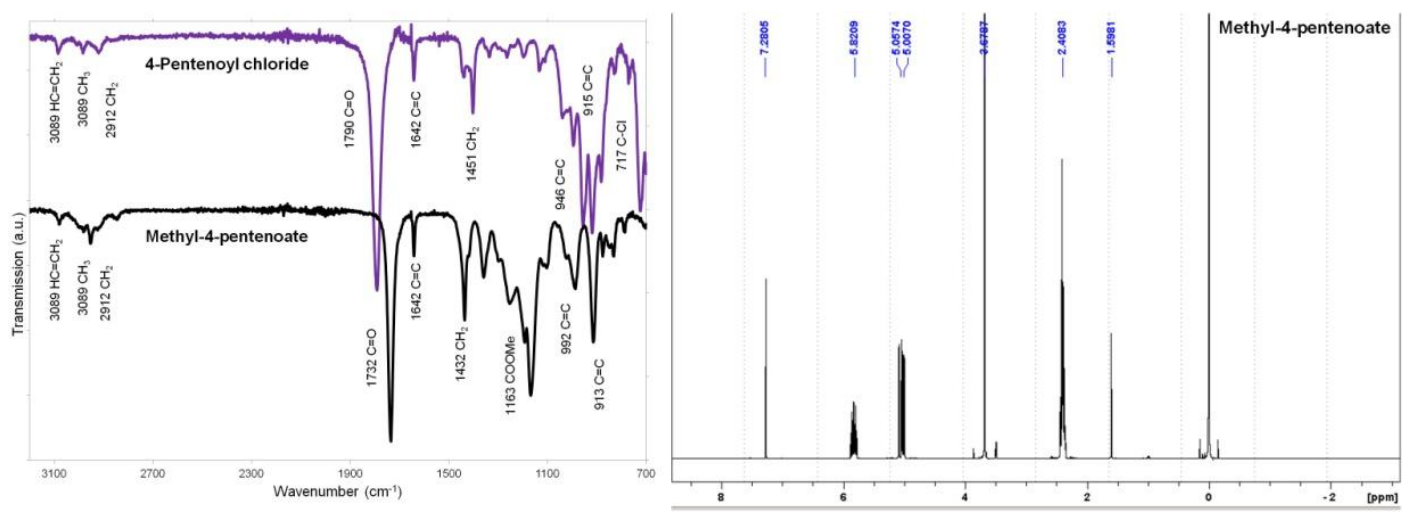

Figure E.1. FTIR and NMR of methyl-4-pentenoate.

\section{E.1.2 tert-Butyl-4-pentenoate (5T)}

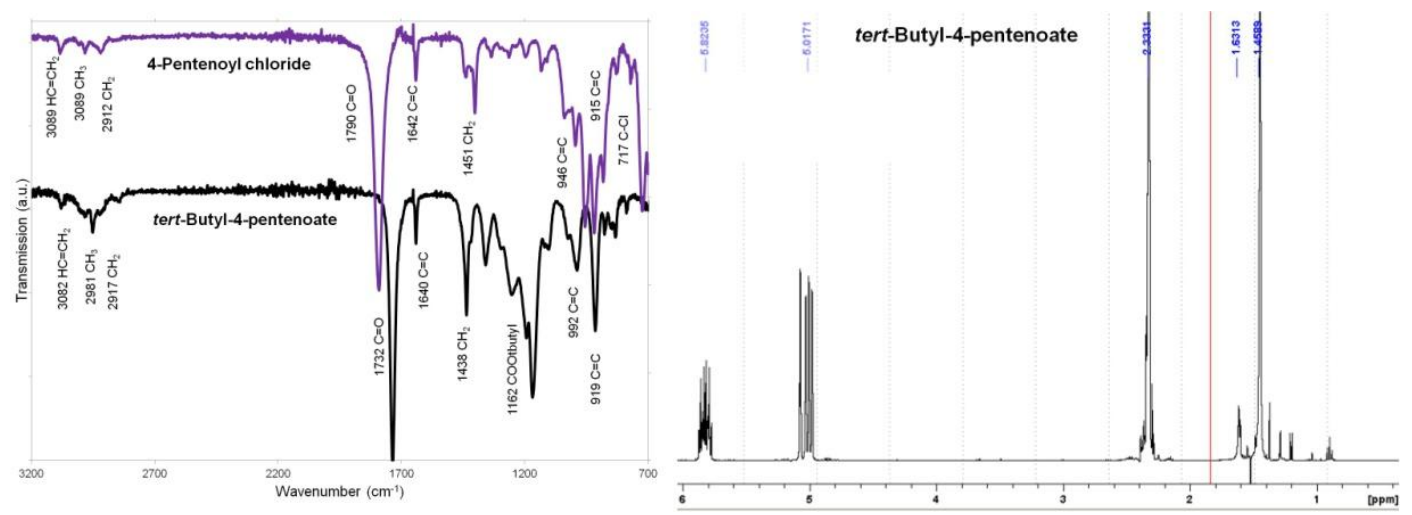

Figure E.2. FTIR and NMR of tert-butyl-4-pentenoate. 


\section{E.1.3 Methyl-10-undecenoate (11M)}

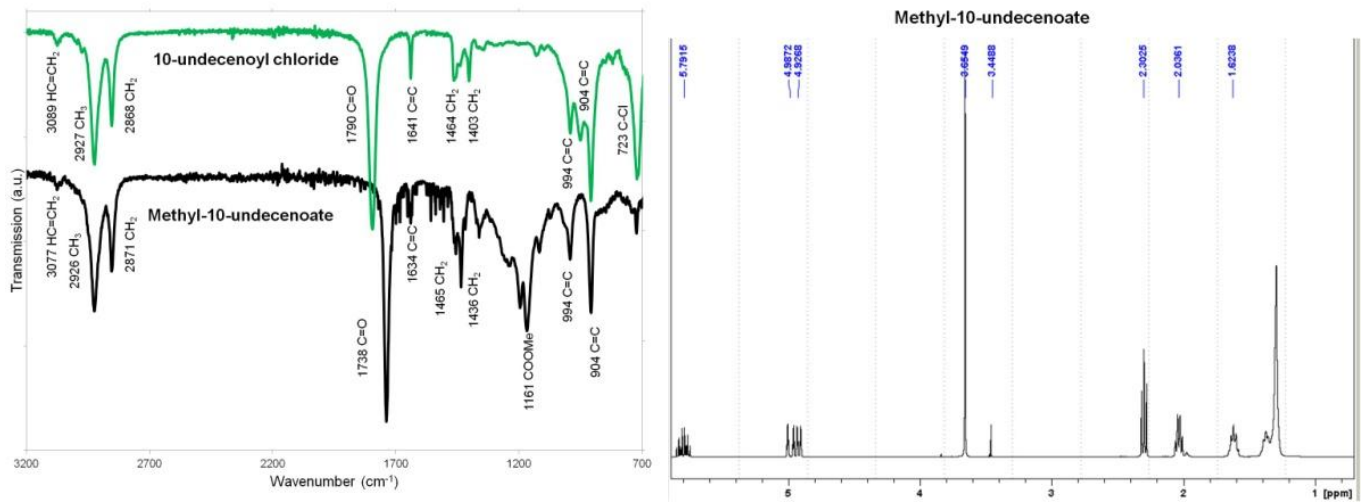

Figure E.3. FTIR and NMR of Methyl-10-undecenoate.

\section{E.1.4 tert-Butyl-10-undecenoate (11T)}
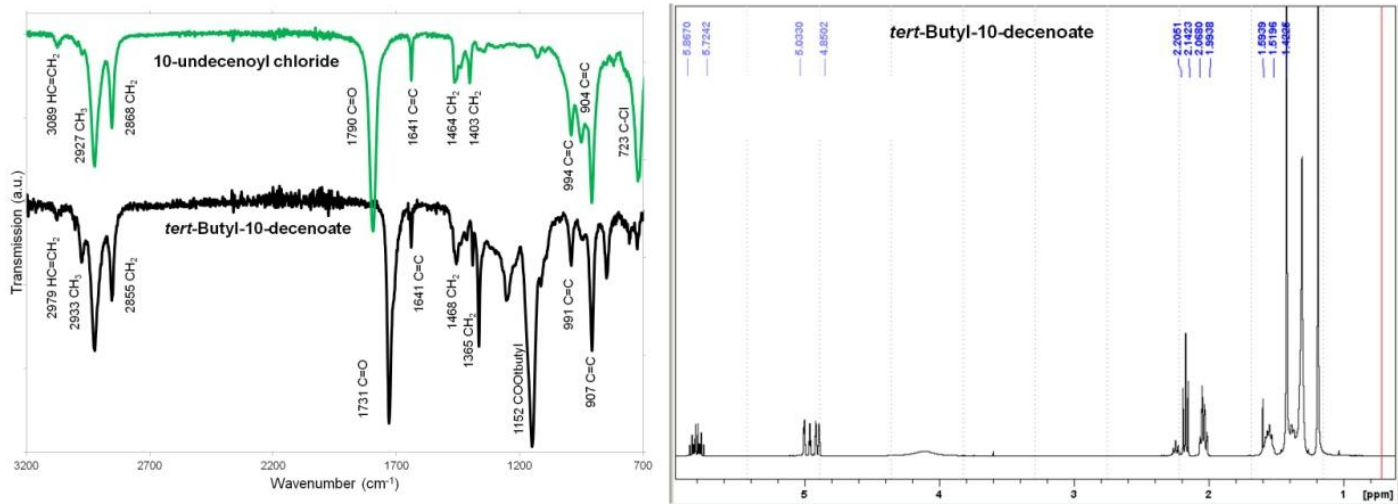

Figure E.4. FTIR and NMR of tert-butyl-10-undecenoate.

\section{E.2 Analytical Characterization of the Ester-Functionalized Si NPs}

\section{E.2.1 Methyl-4-pentenoate-Functinalized Si NPs (5M-Si NPs)}

The successful HS between the Si-H NPs and methyl-4-pentenoate was confirmed by the disappearance of the $\mathrm{C}=\mathrm{C}$ stretches from neat ligand at $1639 \mathrm{~cm}^{-1}, 991 \mathrm{~cm}^{-1}$, and $915 \mathrm{~cm}^{-1}$ and the formation of a covalent $\mathrm{Si}-\mathrm{C}$ stretch at $1268 \mathrm{~cm}^{-1}$ in the methyl-4- 
pentenoate-functionalized $\mathrm{Si}$ NPs $\quad(5 \mathrm{M}-\mathrm{Si}$ NP) FTIR spectrum (Figure E.5). Additionally, the Si-H stretch from the precursor Si NPs at $2100 \mathrm{~cm}^{-1}$ (data not shown) is now absent. The carbonyl stretch of the ester alongside the methyl stretch is observed in the $5 \mathrm{M}-\mathrm{Si}$ NP spectrum at $1727 \mathrm{~cm}^{-1}$ and $1121 \mathrm{~cm}^{-1}$, respectively. Because only stretches attributed to the neat ligand, with the expectation of the Si-C stretch, are observed in the 5M-Si NP spectrum, this suggests that the Si NPs are successfully terminated with methyl-4-pentenoate. It should also be noted that the 5M-Si NPs exhibit little surface oxidation (ca. $1000 \mathrm{~cm}^{-1}$ ).

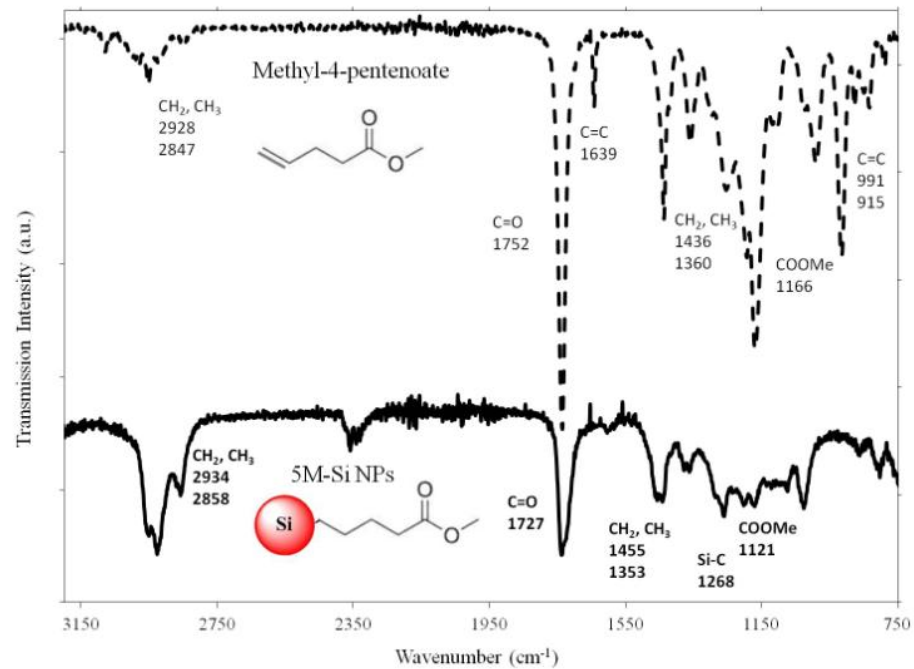

Figure E.5. FTIR of neat methyl-4-pentenoate (top, dashed) and the 5M-Si NPs (bottom, solid). The presence of the $\mathrm{Si}-\mathrm{C}$ stretch and loss of the $\mathrm{C}=\mathrm{C}$ stretches support the HS of the Si-H NPs with the bifunctional ester.

Representative TEM images of the 5M-Si NPs shows spherical and relatively monodisperse NPs (Figure E.6). Though HR-TEM shows NPs with lattice fringes supporting the presence of crystalline particles, a definitive SAED pattern could not be 
obtained; only one diffraction point was observed, potential corresponding to the (111) plane of crystalline Si (3.05 ^ं JCP-DS card no. 001-0787). Counting 408 NPs, an average size of $5.86 \pm 1.40 \mathrm{~nm}$ was found. This represents a small size change from the Si-H NPs $(6.25 \pm 1.71 \mathrm{~nm})$ after HS.
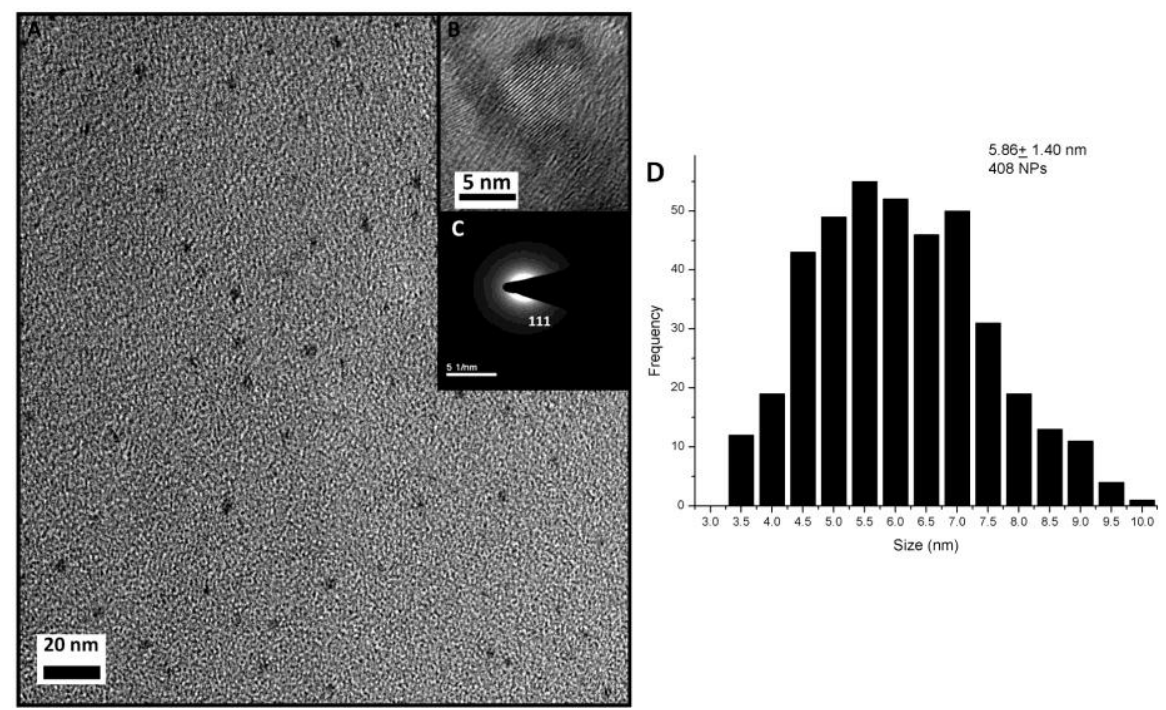

Figure E.6. TEM of 5M-Si NPs. A. TEM and B. HR-TEM images of the 5M-Si NPs showing the presence of spherical, crystalline NPs; C. The SAED pattern was consistent with only the 111 plane of $\mathrm{Si}^{0}$; D. The corresponding histogram of 408 NPs with an average size of $5.86 \pm 1.40 \mathrm{~nm}$.

The prepared Si-H NPs (dispersed in pentane) have an emission maximum at 600 $\mathrm{nm}$ with red/orange emission. After the HS with methyl-4-petenoate, the 5M-Si NPs (in neat xylene or hexanes) exhibit a red/orange color. However, the emission spectrum of the 5M-Si NPs was somewhat complicated; when excited using 340-460 nm light, the Si NP solution exhibit a blue emissive component, which shifts as a function of excitation energy (Figure E.7) The emission maximum of these Si NPs is within this blue component $\left(\lambda_{\mathrm{em}}=450 \mathrm{~nm}\right)$, though the observed emission color is red. All collected 
spectra also have a shoulder at $579 \mathrm{~nm}$ (Fig. E.7), which is close to the emission maximum of the neat Si-H NPs (inset).

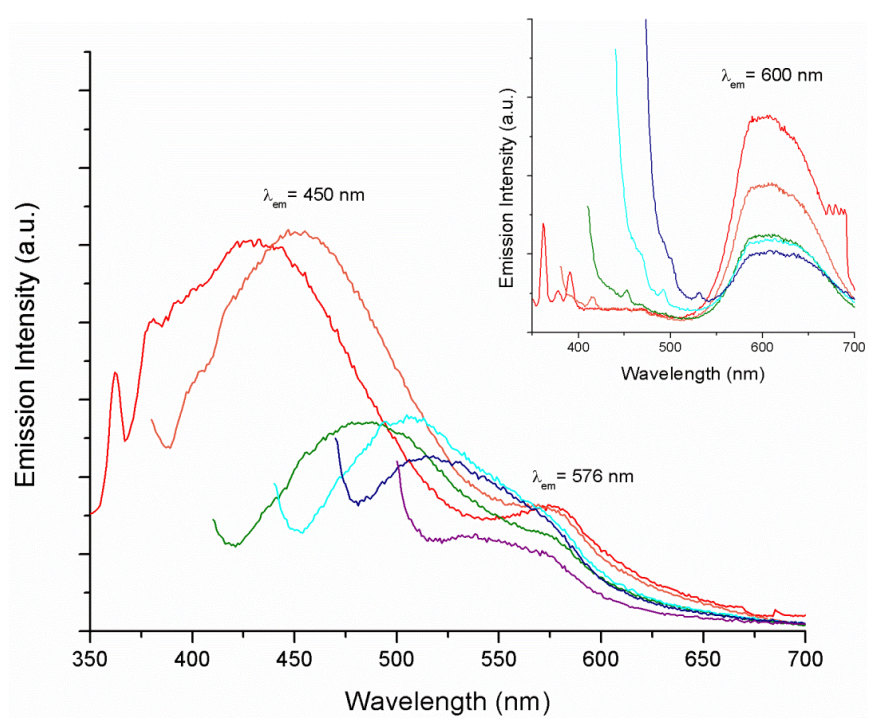

Figure E.7. Emission spectrum of the 5M-Si NPs. Various excitation wavelengths were used (shown 340-4 $90 \mathrm{~nm}$, in $30 \mathrm{~nm}$ intervals). Both a blue emission component $\left(\lambda_{\max }=450 \mathrm{~nm}\right)$ and a red emission component $\left(\lambda_{\max }=576 \mathrm{~nm}\right)$ are observed; Inset. Si-H NP emission spectrum using various excitation wavelengths (340-460 $\mathrm{nm}$, in $30 \mathrm{~nm}$ intervals).

\section{E.2.2 Methyl-10-undecenoate-Functionalized Si NPs (11M-Si NPs)}

The HS of methyl-10-undecenoate and the Si-H NPs are successfully produced Methyl-10-undecenoate-functionalized Si NPs (11M-Si NPs). The product solution showed a Si-C stretch is observed at $1256 \mathrm{~cm}^{-1}$ alongside the carbonyl $\left(1738 \mathrm{~cm}^{-1}\right)$ and methoxy $\left(1090 \mathrm{~cm}^{-1}\right)$ stretches in the FT-IR (Figure E.8). However, even in hexane, the 11M-Si NPs exhibit some Si-O stretches at $1059 \mathrm{~cm}^{-1}$ and $791 \mathrm{~cm}^{-1}$. Some residual neat methyl-10-undecenoate stretches are also observed at $1639 \mathrm{~cm}^{-1} \mathrm{~cm}, 995 \mathrm{~cm}^{-1}$, and 906 $\mathrm{cm}^{-1}$; the boiling point of methyl-10-undecenoate is approximately $245^{\circ} \mathrm{C}$, making it 
difficult to removal, even under decreased pressure. Possible competition for the surface sites between the alkene and ester carbonyl may also be present due to the extended chain length.

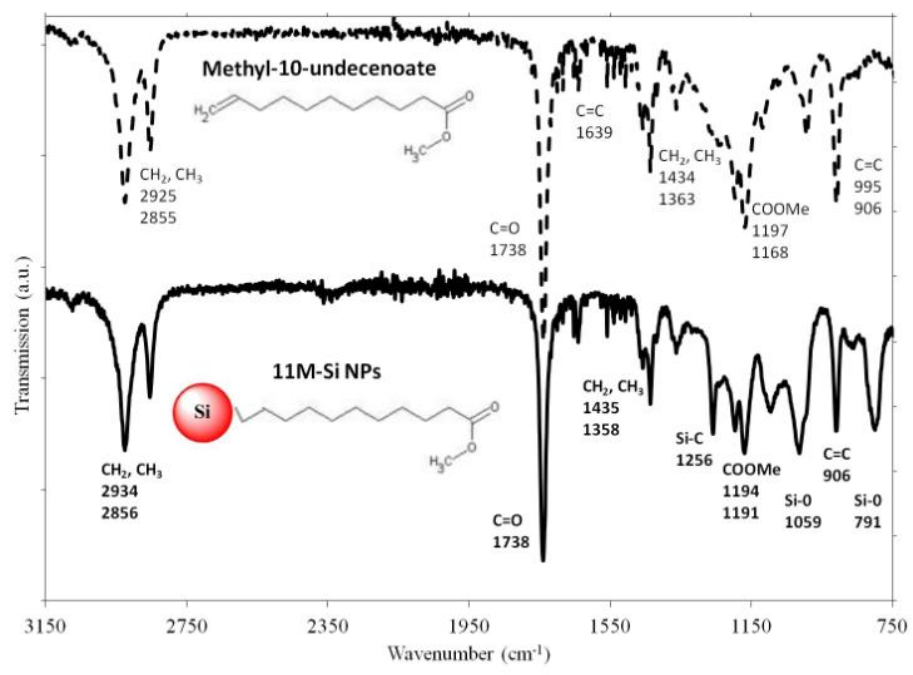

Figure E.8. FTIR of methyl-10-undecenoate (top, dashed) compared to 11M-Si NPs (bottom solid).

The presence of a $\mathrm{Si}-\mathrm{C}$ stretch supports the successful HS of the $\mathrm{Si}-\mathrm{H}$ NPs with the bifunctional ester, though some surface oxidation has been observed.

TEM on the 11M-Si NP solution (Figure E.9,) show readily dispersed NPs. HRTEM shows that the spherical 11M-Si NPs exhibit lattice fringes, which supports the presence of crystalline $\mathrm{Si}^{0}$. Additionally, the SAED pattern further confirms that the 11M-Si NPs consist of crystalline Si, with (111) and (220) diffraction planes $(3.11 \AA$ and $1.93 \AA$, respectively, JCPDS card no. 001-0787). However, the presence of the 111 diffraction pattern (marked with *) is due to the presence of $\mathrm{SiO}_{2}$ (JCPDS 087-2096), supporting Si-O stretch in the FT-IR. An average size of $5.16 \pm 1.22 \mathrm{~nm}$ was found from counting 503 NPs (Fig. E.9). 

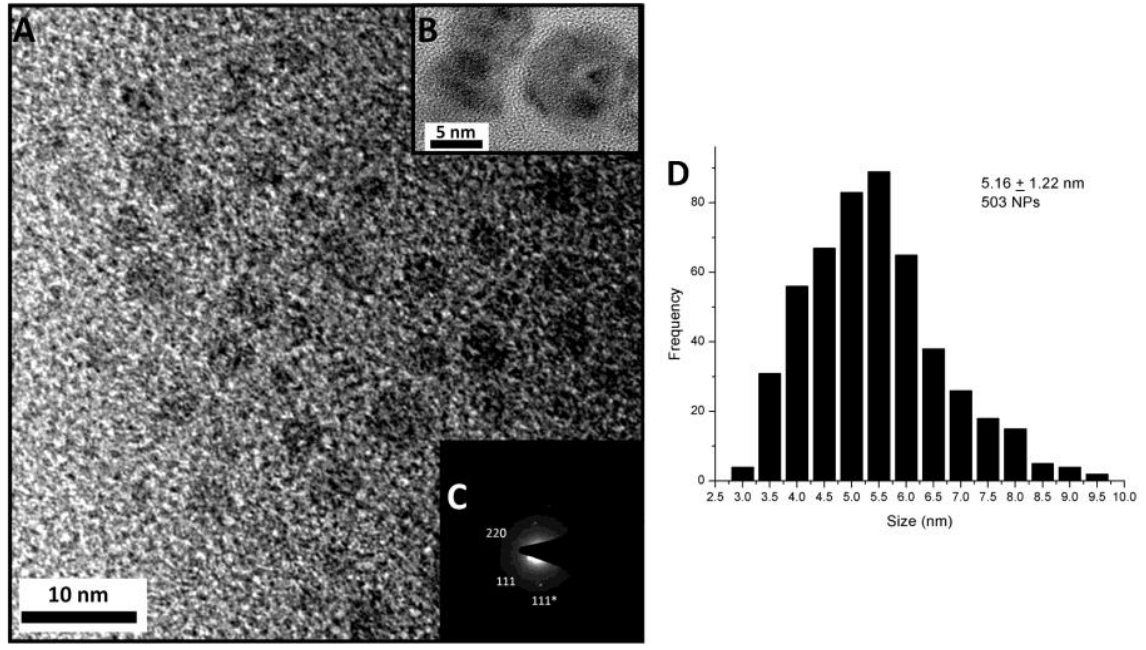

Size $(n m)$

Figure E.9. TEM of 11M-Si NPs. A. TEM and B. HR-TEM images of the 11M-Si NPs illustrating the NPs are spherical and crystalline. C. The SAED pattern was consistent with the (111) and (220) planes of $\mathrm{Si}^{0}$. D. The corresponding histogram of $503 \mathrm{NPs}$; the average $5 \mathrm{M}-\mathrm{Si}$ NP was $5.16 \pm 1.220 \mathrm{~nm}$.

Similar to the 5M-Si NPs, the neat emission spectra of the 11M-Si NPs exhibited an orange/red color when observed by a handheld UV lamp. Spectroscopically, the 11MSi NP solutions exhibit an emission spectra with a blue emission maximum (Figure E.10, $\left.\lambda_{\mathrm{em}}=450 \mathrm{~nm}\right)$ that is also dependent on the excitation wavelength $(340-490 \mathrm{~nm})$. All of the excitation wavelengths used reveal an emission peak at $576 \mathrm{~nm}$, likely responsible for the observed red/orange emission. 


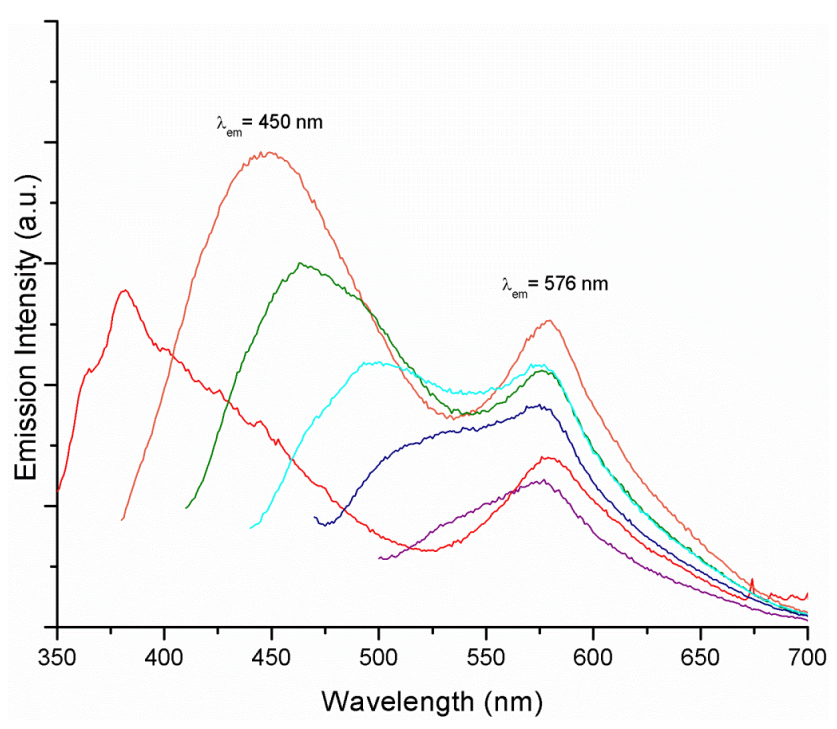

Figure E.10. Emission spectrum of the 11M-Si NPs. Various excitation wavelengths were used (shown $340-490 \mathrm{~nm}$, in $30 \mathrm{~nm}$ intervals). Both a blue emission component $\left(\lambda_{\mathrm{em}}=450 \mathrm{~nm}\right)$ want a red emission component $\left(\lambda_{\mathrm{em}}=576 \mathrm{~nm}\right)$ are observed.

\section{E.2.3 tert-Butyl-4-pentenoate-Functionalized Si NPs (5T-Si NPs)}

The successful HS of the Si-H NPs and tert-butyl-4-pentenoate was confirmed by FT-IR. The 5T-Si NP spectrum (Figure. E.11) shows the presence of a carbonyl (1731 $\left.\mathrm{cm}^{-1}\right)$ and a strong t-butyl ester stretch $\left(1124 \mathrm{~cm}^{-1}\right)$ from the ligand. Due to the bulk of the t-butyl group, the formation of the Si-C stretch at $1253 \mathrm{~cm}^{-1}$ slightly red shifts the tbutyl. Some surface Si-O stretches are observed from the broad nature of the t-butyl stretch, as well as new peaks at $1017 \mathrm{~cm}^{-1}$ and $790 \mathrm{~cm}^{-1}$. 


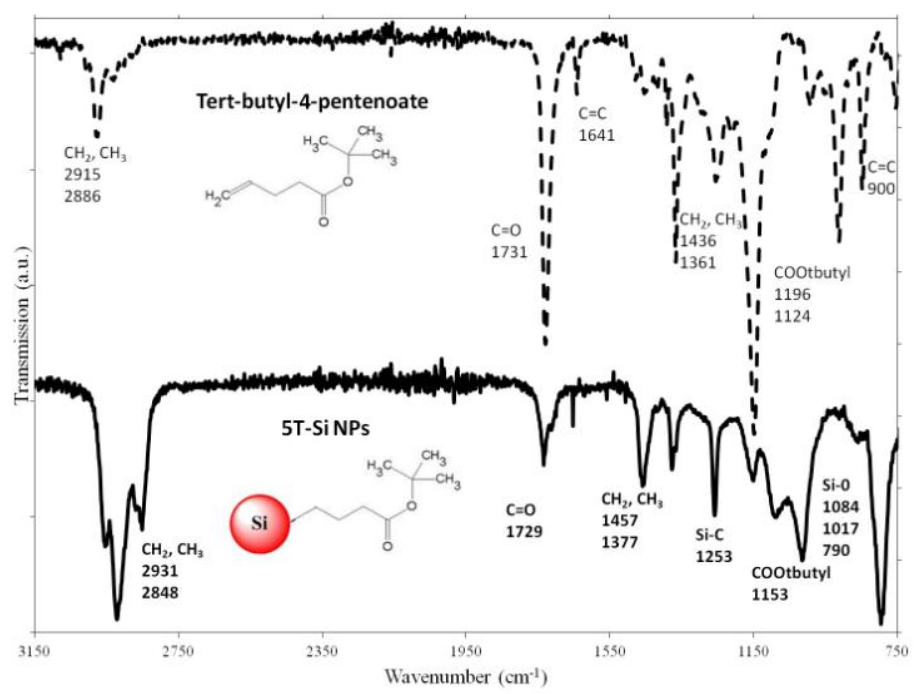

Figure E.11. FTIR of tert-butyl-4-pentenoate (top, dashed) compared to 5T-Si NPs (bottom solid). The Si-C stretch supports the successful HS of the Si-H NPs with the bifunctional ester with some surface oxidation.

Figure E.12 shows that the TEM of the 5T-Si NPs are spherical with multiple facets. Though the FT-IR suggests some surface oxidation, the SAED pattern only reveals reflections of the (111) and (220) planes of crystalline $\mathrm{Si}$ (no $\mathrm{SiO}_{2}$ ). These particles had an average size of $5.42 \pm 1.31 \mathrm{~nm}$ (528 NPs counted). 

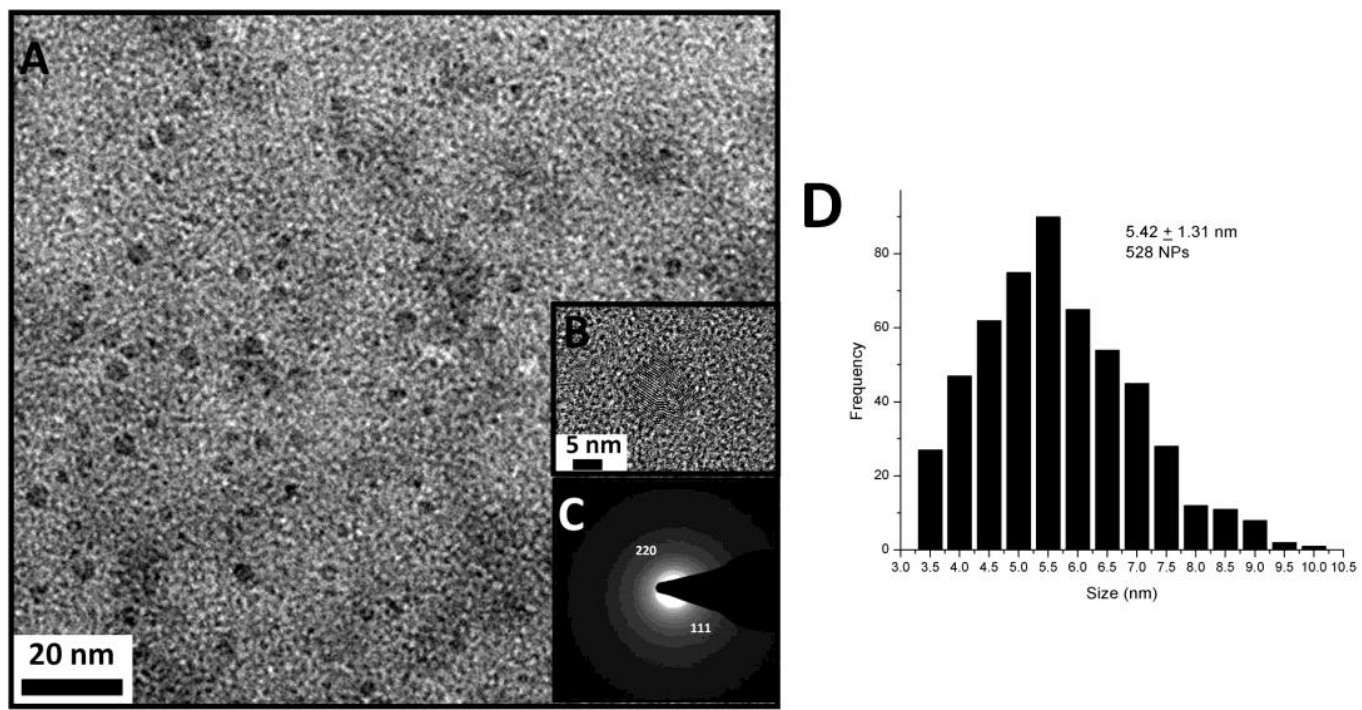

Figure E.12. A., B. TEM of the 5T-Si NPs, illustrating the crystallinity and multiple facers of the 5T-Si NPs; C. The SAED pattern supported the presence of the (111) and (220) $\mathrm{Si}^{0}$ diffraction planes; D.The average particle size, $5.4 \pm 1.31 \mathrm{~nm}$, was determined from counting $528 \mathrm{NPs}$.

The neat 5T-Si NPs possess a blue emission $\left(\lambda_{\mathrm{em}}=440 \mathrm{~nm}, \lambda_{\mathrm{em}}=340-460 \mathrm{~nm}\right)$ that shifts as a function of excitation energy (Figure E.13). All emission spectra have a shoulder at $574 \mathrm{~nm}$. 


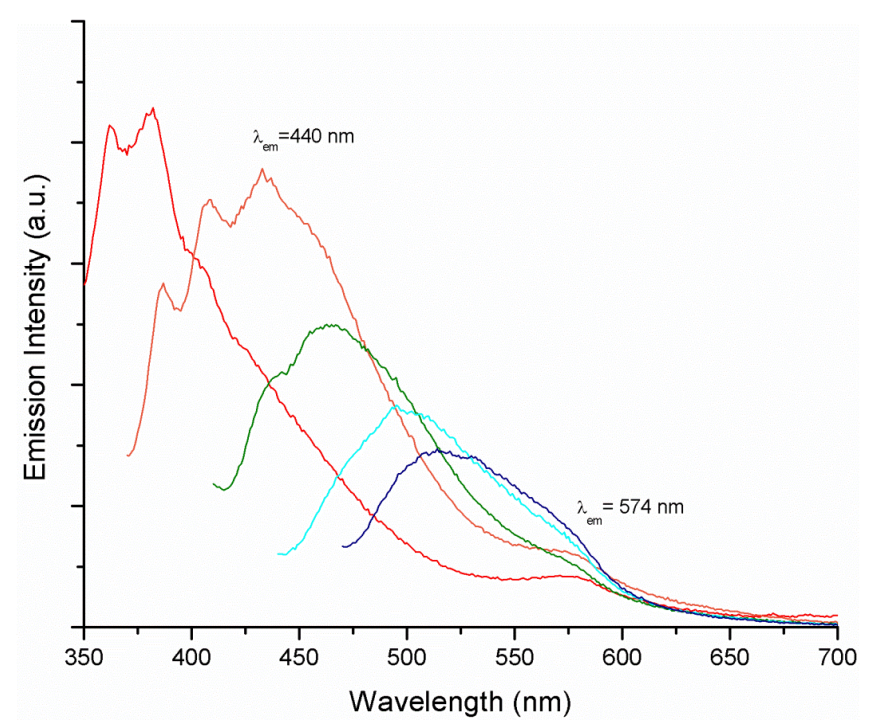

Figure E.13. Emission spectrum of the 5T-Si NPs. Various excitation wavelengths were used (shown $340-460 \mathrm{~nm}$, in $30 \mathrm{~nm}$ intervals). Both a blue emission component $\left(\lambda_{\text {emx }}=440 \mathrm{~nm}\right)$ and a red emission component $\left(\lambda_{\mathrm{ex}}=574 \mathrm{~nm}\right)$ are observed.

\section{E.2.4 tert-Butyl-10-decenoate-Functinalized Si NPs (11T-Si NPs)}

The tert-butyl-10-pentenoate-functionalized Si NPs were found to have a Si-C stretch at $1228 \mathrm{~cm}^{-1}$ (Figure. E.14). Interestingly the carbonyl appears split at $1734 \mathrm{~cm}^{-1}$ and $1712 \mathrm{~cm}^{-1}$. The $\mathrm{C}=\mathrm{C}$ stretches are still observable at $1630 \mathrm{~cm}^{-1}, 990 \mathrm{~cm}^{-1}$, and 909 $\mathrm{cm}^{-1}$, which is likely due to the elevated boiling point of the neat ligand, making complete removal difficult. It is likely that the carbonyl stretch at $1734 \mathrm{~cm}^{-1}$ is that of the neat ligand $\left(1738 \mathrm{~cm}^{-1}\right)$, but that the carbonyl at $1712 \mathrm{~cm}^{-1}$ present in the ligand that is interacts with the surface of the Si NP. A strong t-butyl ester stretch, observed at 1150 $\mathrm{cm}^{-1}$ and $1101 \mathrm{~cm}^{-1}$, represents the neat ligand and the bound ligand, respectively. Slight surface is observed from the broad nature of the t-butyl stretch, as well as new peaks at $1000 \mathrm{~cm}^{-1}$ and $804 \mathrm{~cm}^{-1}$. 


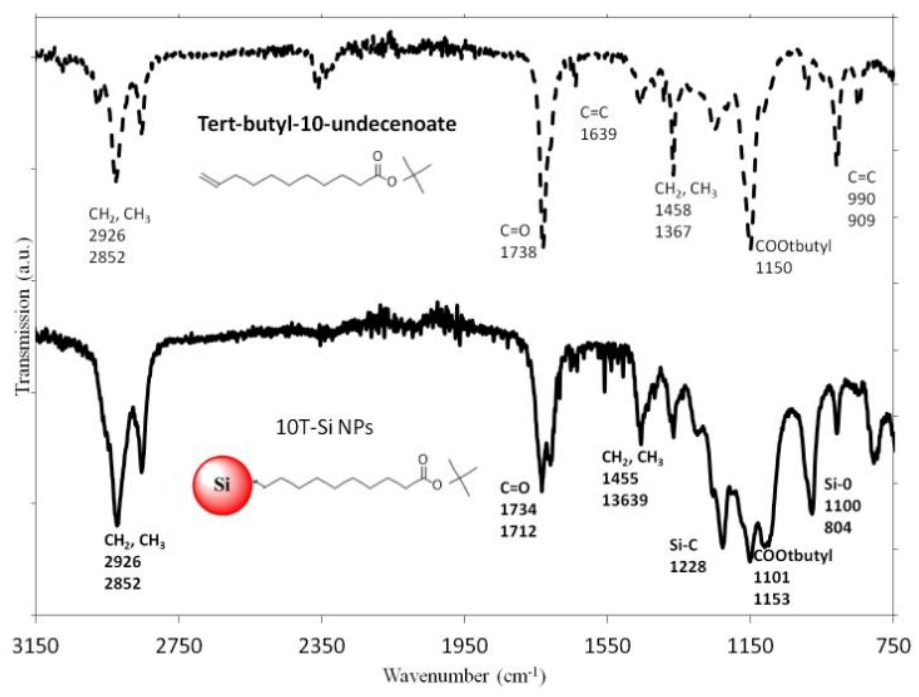

Figure. E.14. FTIR of tert-butyl-10-pentenoate (top, dashed) compared to 11T-Si NPs (bottom solid). The Si-C stretch supports the successful HS of the Si-H NPs with the bi-functional ester with some surface oxidation.

TEM images of the 11T-Si NPs (Figure E.15 A and B) reveal spherical and crystalline NPs. Though the FT-IR suggests that the surface of the 11T-Si NPs possess some surface oxidation, the SAED pattern reveals multiple reflections consistent with $\mathrm{Si}^{0}$, including the (111), (220), and (400) planes, but no $\mathrm{SiO}_{2}$. These particles were also approximately the same size as the other ester-synthesized Si NPs, with an average size of $5.60+1.45 \mathrm{~nm}(523 \mathrm{NPs}$ counted). 

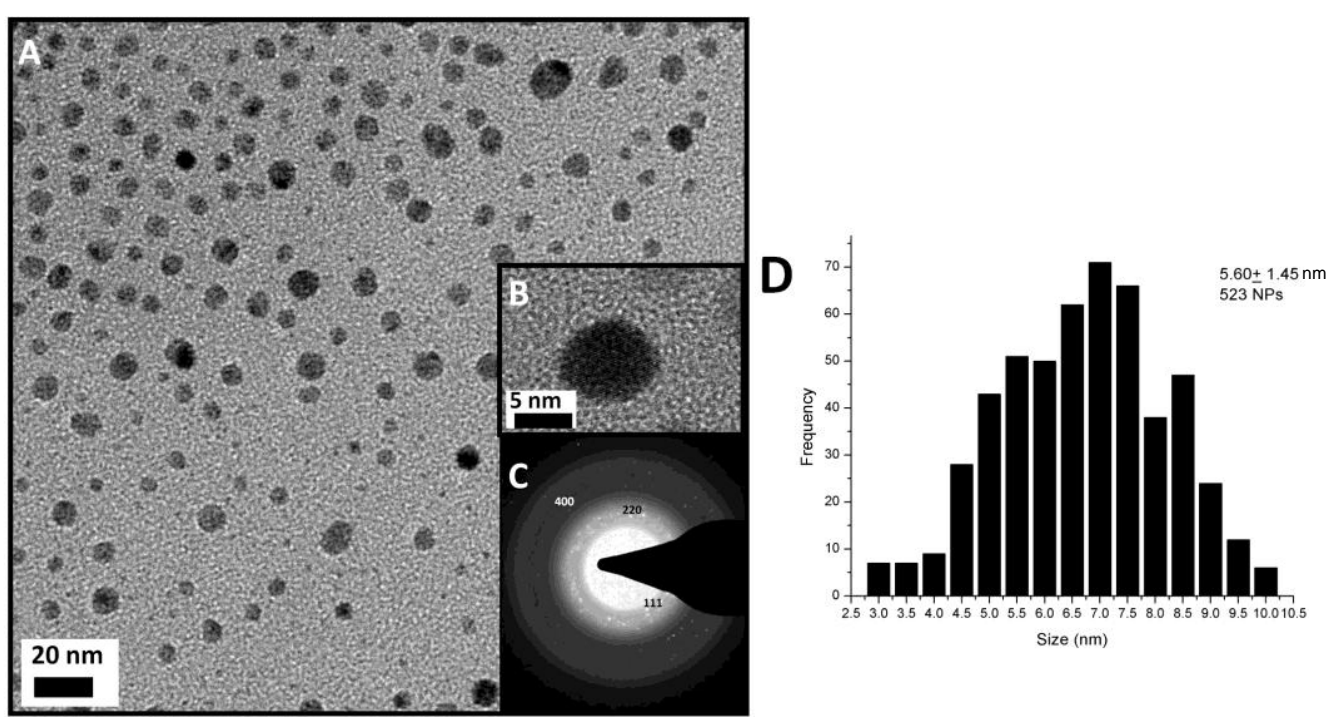

Figure E.15. A., B. TEM of the 11T-Si NPs illustrating the morphology and crystallinity; C. The SAED pattern supporteded with the presence of the $\mathrm{Si}^{0}$ diffraction planes ((111), (220), and (400); D. The average particle size, $5.6 \pm 1.45 \mathrm{~nm}$, was determined from counting 523 NPs.

Similar to the other emission spectra of the ester-synthesized Si NPs, the neat 11T-Si NPs $\left(\lambda_{\mathrm{ex}}=340-460 \mathrm{~nm}\right)$ possess both a blue and red emission component (Figure. E.16). The blue emission maximum $\left(\lambda_{\mathrm{em}}=526 \mathrm{~nm}\right)$ does not significantly shift with varying excitation energy, while the red emission maximum $(570 \mathrm{~nm})$ does not. 


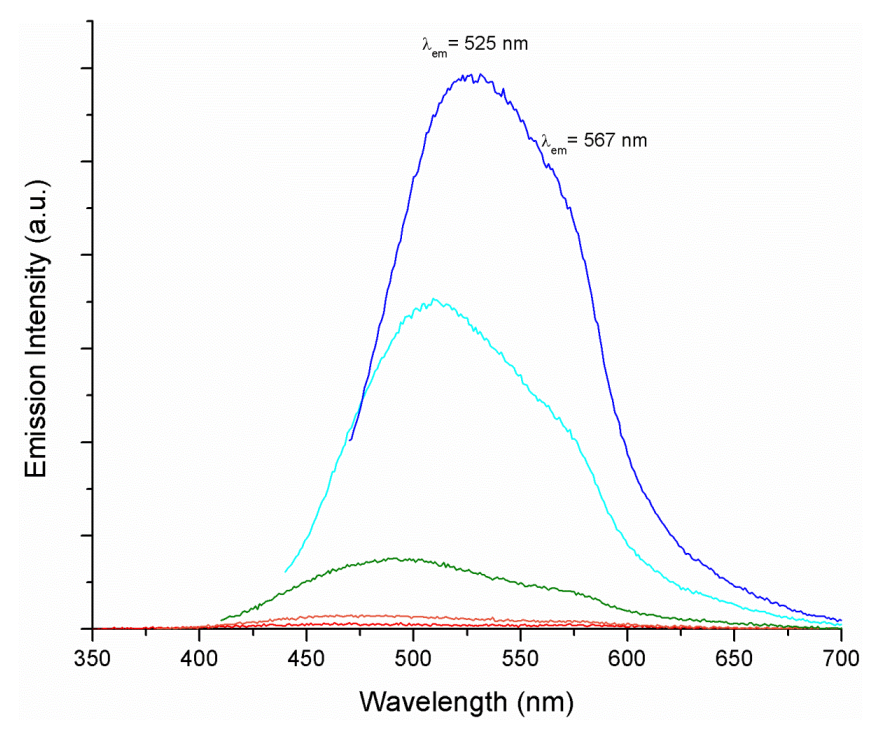

Figure E.16. Emission spectrum of the 11T-Si NPs. Various excitation wavelengths were used (shown $340-460 \mathrm{~nm}$, in $30 \mathrm{~nm}$ intervals). A strong blue emission component $\left(\lambda_{\mathrm{emx}}=5250 \mathrm{~nm}\right)$ and a small red shoulders $\left(\lambda_{\mathrm{ex}}=567 \mathrm{~nm}\right)$ are observed.

\section{E.3. Synthesis of Carboxyl-Functionalized Si NPs from 5M-Si NPs}

The carboxyl-functionalized Si NPs were prepared from the 5M-Si NP solution through base hydrolysis in water based on a previously reported method. ${ }^{[1]}$ The $5 \mathrm{M}-\mathrm{Si}$ NPs were brought up in $7.5 \mathrm{~mL}$ methanol and $22.5 \mathrm{~mL}$ water (1:3) with $\mathrm{NaOH}(7.5 \mathrm{wt}$ $\%$ ). The solution was heated to $50{ }^{\circ} \mathrm{C}$ and stirred for $1 \mathrm{hr}$. The solvent was removed by rotary evaporation and $20 \mathrm{~mL}$ THF was added, and the solution was sonicated for 40 mins. After filtering, the insoluble component was dissolved in $\mathrm{CH}_{2} \mathrm{Cl}_{2}$. An extraction with water was performed, and the obtained water solution was collected.

To confirm the successful hydrolysis, the FT-IR spectrum of the COOH-Si NPs in water were compared to the FT-IR spectrum of the 5M-Si NPs in methanol. As shown in Figure E.17A, both spectra show a Si-C stretch at 1262 and $1252 \mathrm{~cm}^{-1}$, respectively. 
The methoxy ester stretch $\left(1163 \mathrm{~cm}^{-1}\right)$ is no longer observable, and a potential $\mathrm{C}-\mathrm{O}$ a stretch is present around $1081 \mathrm{~cm}^{-1}$. Additionally, a broad $\mathrm{OH}$ stretch $\left(3459 \mathrm{~cm}^{-1}\right)$ and a red shifted $\mathrm{C}=\mathrm{O}$ stretch $\left(1726 \mathrm{~cm}^{-1}\right)$ consistent with a carboxylic acid are observed. Alongside the Si-O stretches around $1000 \mathrm{~cm}^{-1}$ and $793 \mathrm{~cm}^{-1}$, this suggests that the hydrolysis of the 5M-Si NPs was successful but also leads to partial surface oxidation.
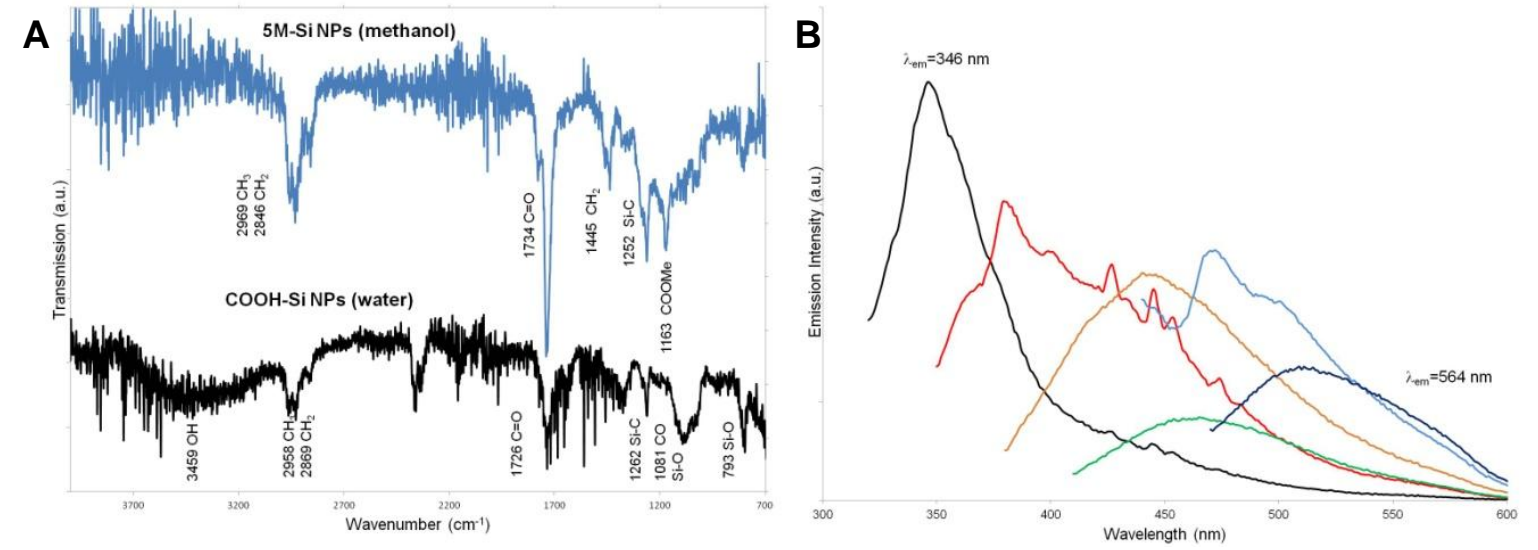

Figure E.17. A. FT-IR of the 5M-Si NPs before (in methanol) and after (water) hydrolysis. B. Emission spectrum of the COOH-Si NPs. Various excitation wavelengths were used (shown 310-460 nm, in $30 \mathrm{~nm}$ intervals). A blue emission is observed $\left(\lambda_{\mathrm{emx}}=346 \mathrm{~nm}\right)$, with a small potential red shoulders $\left(\lambda_{\mathrm{ex}}=564 \mathrm{~nm}\right)$.

The observed $\mathrm{COOH}-\mathrm{Si}$ NPs in water was found to emit blue, with an emission maximum of $346 \mathrm{~nm}$ when excited with $310 \mathrm{~nm}$ light (Fig. E.17B). This emission has a dependence on the excitation wavelength. Interestingly, a potential shoulder is still observable at $564 \mathrm{~nm}$ when low energy wavelengths are used. 